\title{
Impacto da geração de grafos na classificação semissupervisionada
}

Celso André Rodrigues de Sousa 

SERVIÇO DE PÓS-GRADUAÇÃO DO ICMC-USP

Data de Depósito:

Assinatura:

\title{
Impacto da geração de grafos na classificação semissupervisionada
}

\author{
Celso André Rodrigues de Sousa
}

Orientador: Prof. Dr. Alneu de Andrade Lopes

Dissertação apresentada ao Instituto de Ciências Matemáticas e de Computação - ICMC-USP, como parte dos requisitos para obtenção do título de Mestre em Ciências - Ciências de Computação e Matemática Computacional. EXEMPLAR DE DEFESA. 
Ficha catalográfica elaborada pela Biblioteca Prof. Achille Bassi e Seção Técnica de Informática, ICMC/USP,

com os dados fornecidos pelo(a) autor(a)

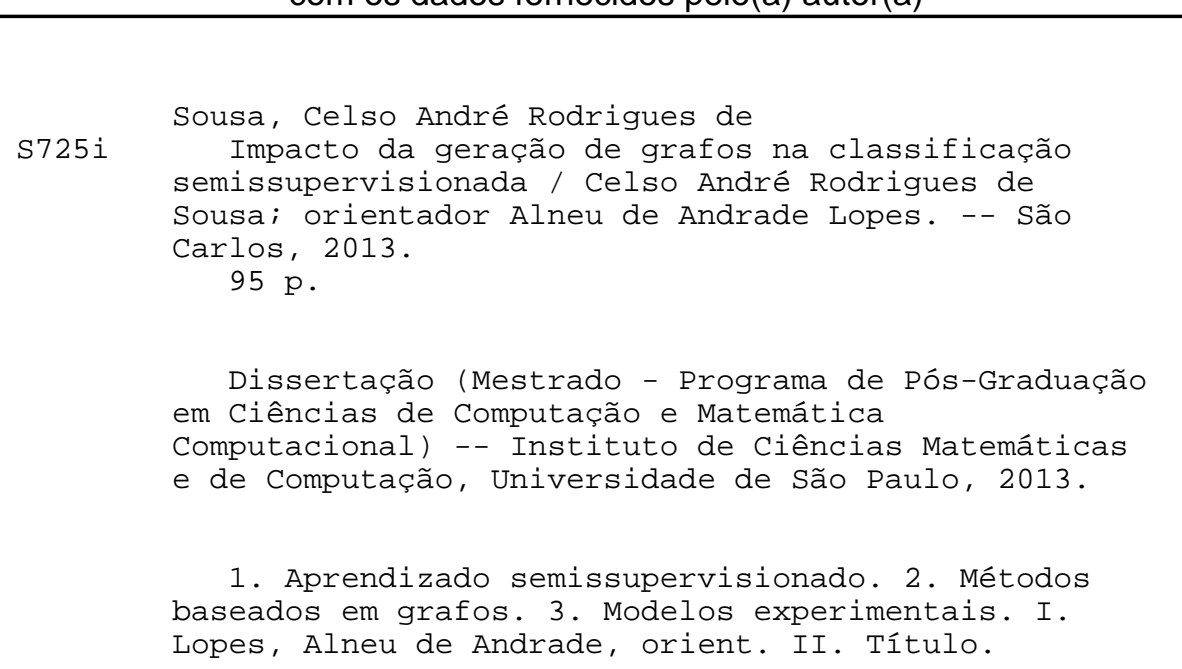




\section{Resumo}

Uma variedade de algoritmos de aprendizado semissupervisionado baseado em grafos e métodos de geração de grafos foram propostos pela comunidade científica nos últimos anos. Apesar de seu aparente sucesso empírico, a área de aprendizado semissupervisionado carece de um estudo empírico detalhado que avalie o impacto da geração de grafos na classificação semissupervisionada. Neste trabalho, é provido tal estudo empírico. Para tanto, combinam-se uma variedade de métodos de geração de grafos com uma variedade de algoritmos de aprendizado semissupervisionado baseado em grafos para comparálos empiricamente em seis bases de dados amplamente usadas na literatura de aprendizado semissupervisionado. Os algoritmos são avaliados em tarefas de classificação de dígitos, caracteres, texto, imagens e de distribuições gaussianas. A avaliação experimental proposta neste trabalho é subdividida em quatro partes: (1) análise de melhor caso; (2) avaliação da estabilidade dos classificadores semissupervisionados; (3) avaliação do impacto da geração de grafos na classificação semissupervisionada; (4) avaliação da influência dos parâmetros de regularização no desempenho de classificação dos classificadores semissupervisionados. Na análise de melhor caso, avaliam-se as melhores taxas de erro de cada algoritmo semissupervisionado combinado com os métodos de geração de grafos usando uma variedade de valores para o parâmetro de esparsificação, o qual está relacionado ao número de vizinhos de cada exemplo de treinamento. Na avaliação da estabilidade dos classificadores, avalia-se a estabilidade dos classificadores semissupervisionados combinados com os métodos de geração de grafos usando uma variedade de valores para o parâmetro de esparsificação. Para tanto, fixam-se os valores dos parâmetros de regularização (quando existirem) que geraram os melhores resultados na análise de melhor caso. Na avaliação do impacto da geração de grafos, avaliam-se os métodos de geração de grafos combinados com os algoritmos de aprendizado semissupervisionado usando uma variedade de valores para o parâmetro de esparsificação. Assim como na avaliação da estabilidade dos classificadores, para esta avaliação, fixam-se os valores dos parâmetros de regularização (quando existirem) que geraram os melhores resultados na análise de melhor caso. Na avaliação da influência dos parâmetros de regularização na classificação semissupervisionada, avaliam-se as superfícies de erro geradas pelos classificadores semissupervisionados em cada grafo e cada 
base de dados. Para tanto, fixam-se os grafos que geraram os melhores resultados na análise de melhor caso e variam-se os valores dos parâmetros de regularização. O intuito destes experimentos é avaliar o balanceamento entre desempenho de classificação e estabilidade dos algoritmos de aprendizado semissupervisionado baseado em grafos numa variedade de métodos de geração de grafos e valores de parâmetros (de esparsificação e de regularização, se houver). A partir dos resultados obtidos, pode-se concluir que o grafo $k$ vizinhos mais próximos mútuo (mutKNN) pode ser a melhor opção dentre os métodos de geração de grafos de adjacência, enquanto que o kernel RBF pode ser a melhor opção dentre os métodos de geração de matrizes ponderadas. Em adição, o grafo mutKNN tende a gerar superfícies de erro que são mais suaves que aquelas geradas pelos outros métodos de geração de grafos de adjacência. Entretanto, o grafo mutKNN é instável para valores relativamente pequenos de $k$. Os resultados obtidos neste trabalho indicam que o desempenho de classificação dos algoritmos semissupervisionados baseados em grafos é fortemente influenciado pela configuração de parâmetros. Poucos padrões evidentes foram encontrados para auxiliar o processo de seleção de parâmetros. As consequências dessa instabilidade são discutidas neste trabalho em termos de pesquisa e aplicações práticas. 


\section{Abstract}

A variety of graph-based semi-supervised learning algorithms have been proposed by the research community in the last few years. Despite its apparent empirical success, the field of semi-supervised learning lacks a detailed empirical study that evaluates the influence of graph construction on semisupervised learning. In this work we provide such an empirical study. For such purpose, we combine a variety of graph construction methods with a variety of graph-based semi-supervised learning algorithms in order to empirically compare them in six benchmark data sets widely used in the semi-supervised learning literature. The algorithms are evaluated in tasks about digit, character, text, and image classification as well as classification of gaussian distributions. The experimental evaluation proposed in this work is subdivided into four parts: (1) best case analysis; (2) evaluation of classifiers' stability; (3) evaluation of the influence of graph construction on semi-supervised learning; (4) evaluation of the influence of regularization parameters on the classification performance of semi-supervised learning algorithms. In the best case analysis, we evaluate the lowest error rates of each semi-supervised learning algorithm combined with the graph construction methods using a variety of sparsification parameter values. Such parameter is associated with the number of neighbors of each training example. In the evaluation of classifiers' stability, we evaluate the stability of the semi-supervised learning algorithms combined with the graph construction methods using a variety of sparsification parameter values. For such purpose, we fixed the regularization parameter values (if any) with the values that achieved the best result in the best case analysis. In the evaluation of the influence of graph construction, we evaluate the graph construction methods combined with the semi-supervised learning algorithms using a variety of sparsification parameter values. In this analysis, as occurred in the evaluation of classifiers' stability, we fixed the regularization parameter values (if any) with the values that achieved the best result in the best case analysis. In the evaluation of the influence of regularization parameters on the classification performance of semi-supervised learning algorithms, we evaluate the error surfaces generated by the semi-supervised classifiers in each graph and data set. For such purpose, we fixed the graphs that achieved the best results in the best case analysis and varied the regularization parameters' values. The intention of our experiments is evaluating 
the trade-off between classification performance and stability of the graphbased semi-supervised learning algorithms in a variety of graph construction methods as well as parameter values (sparsification and regularization, if applicable). From the obtained results, we conclude that the mutual $k$-nearest neighbors (mutKNN) graph may be the best choice for adjacency graph construction while the RBF kernel may be the best choice for weighted matrix generation. In addition, mutKNN tends to generate error surfaces that are smoother than those generated by other adjacency graph construction methods. However, mutKNN is unstable for relatively small values of $k$. Our results indicate that the classification performance of the graph-based semi-supervised learning algorithms are heavily influenced by parameter setting. We found just a few evident patterns that could help parameter selection. The consequences of such instability are discussed in this work in research and practice. 


\section{Sumário}

Resumo ....................... i i

Abstract .............................. ii

Sumário ....................... . . .

Lista de Figuras . . . . . . . . . . . . . . . . . vii

Lista de Tabelas .................... . . . xi

1 Introdução 1

1.1 Contribuições . . . . . . . . . . . . . . . . 2

1.2 Discussão dos resultados obtidos . . . . . . . . . . . . . . . . . . . . .

1.3 Consequências dos resultados obtidos . . . . . . . . . . . . . 4

1.4 Organização do Trabalho . . . . . . . . . . . . . 5

2 Classificação semissupervisionada baseada em grafos 7

2.1 Preliminares . . . . . . . . . . . . . . 7

2.2 Geração de grafos de adjacência . . . . . . . . . . . . . 10

2.3 Geração de matrizes ponderadas . . . . . . . . . . . . . . 11

2.4 Classificadores semissupervisionados . . . . . . . . . . 12

2.5 Indução heurística . . . . . . . . . . . . . . 15

3 Protocolo Experimental 17

3.1 Bases de dados e pré-processamento . . . . . . . . . . . . 17

3.2 Configuração experimental . . . . . . . . . . . . . . . . 18

3.3 Configuração de parâmetros ................ 21

3.4 Considerações Finais . . . . . . . . . . . . . . . . . 22

4 Análise de melhor caso 23

4.1 Avaliação dos resultados obtidos . . . . . . . . . . . . . 23

4.2 Considerações finais . . . . . . . . . . . . . . 28

5 Avaliação da estabilidade dos classificadores semissupervisionados 29

5.1 Descrição do modelo experimental proposto . . . . . . . . . . . 29

5.2 Avaliação dos resultados obtidos . . . . . . . . . . . . . 40

5.3 Considerações finais . . . . . . . . . . . . . . . 43 
6 Avaliação da influência da geração de grafos na classificação semissupervisionada

6.1 Descrição do modelo experimental proposto . . . . . . . . . . . . 45

6.2 Avaliação dos resultados obtidos . . . . . . . . . . 53

6.3 Considerações finais . . . . . . . . . . . . . . . 56

7 Avaliação da influência dos parâmetros de regularização na classificação semissupervisionada

7.1 Descrição do modelo experimental proposto . . . . . . . . . . . . 60

7.2 Avaliação dos resultados obtidos . . . . . . . . . . . 85

7.3 Considerações finais . . . . . . . . . . . . . . 86

8 Conclusão $\quad 87$

$\begin{array}{ll}\text { Referências Bibliográficas } & 95\end{array}$ 


\section{Lista de Figuras}

5.1 Padrão de gráfico para a avaliação da estabilidade dos classificadores semissupervisionados. . . . . . . . . . . . . 30

5.2 Estabilidade dos classificadores semissupervisionados para as partições de 10 exemplos rotulados usando o grafo symKNN-RBF. 31

5.3 Estabilidade dos classificadores semissupervisionados para as partições de 100 exemplos rotulados usando o grafo symKNN-RBF. 31

5.4 Estabilidade dos classificadores semissupervisionados para as partições de 10 exemplos rotulados usando o grafo mutKNN-RBF. 32

5.5 Estabilidade dos classificadores semissupervisionados para as partições de 100 exemplos rotulados usando o grafo mutKNN-RBF. 32

5.6 Estabilidade dos classificadores semissupervisionados para as partições de 10 exemplos rotulados usando o grafo symFKNN-RBF. 33

5.7 Estabilidade dos classificadores semissupervisionados para as partições de 100 exemplos rotulados usando o grafo symFKNNRBF. . . . . . . . . . . . . . . . . . . 33

5.8 Estabilidade dos classificadores semissupervisionados para as partições de 10 exemplos rotulados usando o grafo symKNN-HM. 34

5.9 Estabilidade dos classificadores semissupervisionados para as partições de 100 exemplos rotulados usando o grafo symKNN-HM. 34

5.10Estabilidade dos classificadores semissupervisionados para as partições de 10 exemplos rotulados usando o grafo mutKNN-HM.

5.11 Estabilidade dos classificadores semissupervisionados para as partições de 100 exemplos rotulados usando o grafo mutKNN-HM. 35

5.12 Estabilidade dos classificadores semissupervisionados para as partições de 10 exemplos rotulados usando o grafo symFKNN-HM. 36

5.13 Estabilidade dos classificadores semissupervisionados para as partições de 100 exemplos rotulados usando o grafo symFKNN-HM. 36

5.14 Estabilidade dos classificadores semissupervisionados para as partições de 10 exemplos rotulados usando o grafo symKNN-LLE. 37

5.15Estabilidade dos classificadores semissupervisionados para as partições de 100 exemplos rotulados usando o grafo symKNN-LLE. 37

5.16Estabilidade dos classificadores semissupervisionados para as partições de 10 exemplos rotulados usando o grafo mutKNN-LLE. 38 
5.17 Estabilidade dos classificadores semissupervisionados para as partições de 100 exemplos rotulados usando o grafo mutKNN-LLE. 38

5.18Estabilidade dos classificadores semissupervisionados para as partições de 10 exemplos rotulados usando o grafo symFKNN-LLE. 39

5.19Estabilidade dos classificadores semissupervisionados para as partições de 100 exemplos rotulados usando o grafo symFKNNLLE. . . . . . . . . . . . . . . . . . . . . . . .

6.1 Padrão de gráfico para a avaliação da influência da geração de grafos na classificação semissupervisionada. . . . . . . . . . .

6.2 Avaliação dos métodos de geração de grafos na base de dados USPS usando as partições de 10 exemplos rotulados. . . . . . . 47

6.3 Avaliação dos métodos de geração de grafos na base de dados USPS usando as partições de 100 exemplos rotulados. . . . . . .

6.4 Avaliação dos métodos de geração de grafos na base de dados $\mathrm{COIL}_{2}$ usando as partições de 10 exemplos rotulados. . . . . . . .

6.5 Avaliação dos métodos de geração de grafos na base de dados $\mathrm{COIL}_{2}$ usando as partições de 100 exemplos rotulados. . . . . . .

6.6 Avaliação dos métodos de geração de grafos na base de dados DIGIT-1 usando as partições de 10 exemplos rotulados. . . . . . .

6.7 Avaliação dos métodos de geração de grafos na base de dados DIGIT-1 usando as partições de 100 exemplos rotulados. . . . . .

6.8 Avaliação dos métodos de geração de grafos na base de dados G-241N usando as partições de 10 exemplos rotulados. . . . . . .

6.9 Avaliação dos métodos de geração de grafos na base de dados G-241N usando as partições de 100 exemplos rotulados. . . . . . 50

6.10Avaliação dos métodos de geração de grafos nas bases de dados G-241C usando as partições de 10 exemplos rotulados. . . . . . .

6.11 Avaliação dos métodos de geração de grafos na base de dados G-241C usando as partições de 100 exemplos rotulados. . . . . .

6.12 Avaliação dos métodos de geração de grafos na base de dados TEXT usando as partições de 10 exemplos rotulados. . . . . . . .

6.13Avaliação dos métodos de geração de grafos na base de dados TEXT usando as partições de 100 exemplos rotulados. . . . . . .

7.1 Padrão de gráfico para a avaliação da influência dos parâmetros de regularização na classificação semissupervisionada. . . . . . 60

7.2 Superfícies de erro do algoritmo LapRLS na base de dados USPS para as partições de 10 exemplos rotulados. . . . . . . . . . . 61

7.3 Superfícies de erro do algoritmo LapRLS na base de dados USPS para as partições de 100 exemplos rotulados. . . . . . . . . .

7.4 Superfícies de erro do algoritmo LapSVM na base de dados USPS para as partições de 10 exemplos rotulados. . . . . . . . . . . 63

7.5 Superfícies de erro do algoritmo LapSVM na base de dados USPS para as partições de 100 exemplos rotulados. . . . . . . . . . . 64

7.6 Superfícies de erro do algoritmo LapRLS na base de dados $\mathrm{COIL}_{2}$ para as partições de 10 exemplos rotulados. . . . . . . . . . . . 
7.7 Superfícies de erro do algoritmo LapRLS na base de dados $\mathrm{COIL}_{2}$ para as partições de 100 exemplos rotulados. . . . . . . . . . 66

7.8 Superfícies de erro do algoritmo LapSVM na base de dados $\mathrm{COIL}_{2}$ para as partições de 10 exemplos rotulados. . . . . . . . . . . 67

7.9 Superfícies de erro do algoritmo LapSVM na base de dados $\mathrm{COIL}_{2}$ para as partições de 100 exemplos rotulados. . . . . . . . . . . 68

7.10 Superfícies de erro do algoritmo LapRLS na base de dados DIGIT1 para as partições de 10 exemplos rotulados. . . . . . . . . . . 69

7.11 Superfícies de erro do algoritmo LapRLS na base de dados DIGIT-

1 para as partições de 100 exemplos rotulados. . . . . . . . . 70

7.12 Superfícies de erro do algoritmo LapSVM na base de dados DIGIT-

1 para as partições de 10 exemplos rotulados. . . . . . . . . 71

7.13Superficies de erro do algoritmo LapSVM na base de dados DIGIT-

1 para as partições de 100 exemplos rotulados. . . . . . . . . . 72

7.14 Superfícies de erro do algoritmo LapRLS na base de dados G241C para as partições de 10 exemplos rotulados. . . . . . . . 73

7.15Superfícies de erro do algoritmo LapRLS na base de dados G241C para as partições de 100 exemplos rotulados. . . . . . . . . 74

7.16 Superfícies de erro do algoritmo LapSVM na base de dados G241C para as partições de 10 exemplos rotulados. . . . . . . . . 75

7.17 Superfícies de erro do algoritmo LapSVM na base de dados G241C para as partições de 100 exemplos rotulados. . . . . . . . 76

7.18Superfícies de erro do algoritmo LapRLS na base de dados G$241 \mathrm{~N}$ para as partições de 10 exemplos rotulados. . . . . . . . . 77

7.19Superfícies de erro do algoritmo LapRLS na base de dados G$241 \mathrm{~N}$ para as partições de 100 exemplos rotulados. . . . . . . . 78

7.20 Superfícies de erro do algoritmo LapSVM na base de dados G$241 \mathrm{~N}$ para as partições de 10 exemplos rotulados. . . . . . . . . 79

7.21 Superfícies de erro do algoritmo LapSVM na base de dados G$241 \mathrm{~N}$ para as partições de 100 exemplos rotulados. . . . . . . . 80

7.22 Superfícies de erro do algoritmo LapRLS na base de dados TEXT para as partições de 10 exemplos rotulados. . . . . . . . . . . 81

7.23 Superfícies de erro do algoritmo LapRLS na base de dados TEXT para as partições de 100 exemplos rotulados. . . . . . . . . . . 82

7.24 Superfícies de erro do algoritmo LapSVM na base de dados TEXT para as partições de 10 exemplos rotulados. . . . . . . . . . . . 83

7.25 Superfícies de erro do algoritmo LapSVM na base de dados TEXT para as partições de 100 exemplos rotulados. . . . . . . . . . 84 



\section{Lista de Tabelas}

3.1 Descrição das bases de dados usadas na avaliação experimental deste trabalho. Os símbolos (a) e (r) indicam quais bases de dados são reais $(\mathrm{r})$ e quais são artificiais (a). . . . . . . . . . . . . . . 18

4.1 Médias das taxas de erro (\%) e desvios-padrão (\%) dos classificadores semissupervisionados baseados em grafos nas partições de 10 exemplos rotulados. . . . . . . . . . . . . . . . . 24

4.2 Rankings médios para os métodos de geração de grafos nas partições de 10 exemplos rotulados em relação a cada classificador semissupervisionado e a média geral dos rankings. . . . . . . . . . 24

4.3 Médias das taxas de erro (\%) e desvios-padrão (\%) dos classificadores semissupervisionados baseados em grafos nas partições de 100 exemplos rotulados.

4.4 Rankings médios para os métodos de geração de grafos nas partições de 100 exemplos rotulados em relação a cada classificador semissupervisionado e a média geral dos rankings. . . . . . . . . . 25

4.5 Os piores $(p)$ e os melhores $(m)$ resultados para as partições de 10 e de 100 exemplos rotulados apresentados por Chapelle et al. (2006) para as bases de dados usadas nos experimentos deste trabalho. Os piores e melhores resultados obtidos neste trabalho são reportados por $p^{*}$ e $m^{*}$, respectivamente. . . . . . . . . . 28 



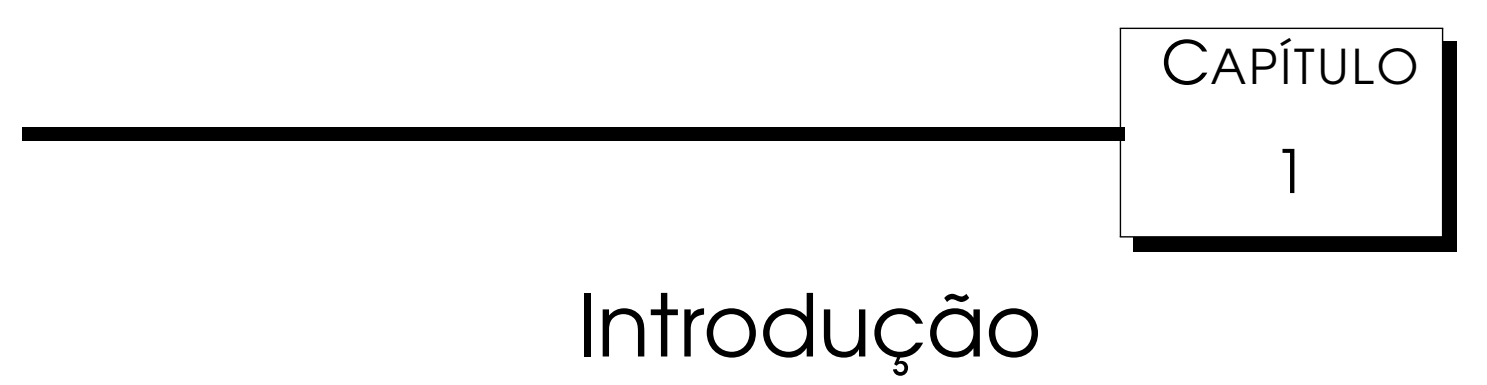

A área de aprendizado semissupervisionado tem recebido um crescente interesse nos últimos anos, tanto do ponto de vista teórico quanto prático (Chapelle et al., 2006; Zhu, 2005; Derbeko et al., 2004; Kääriäinen, 2005; Balcan e Blum, 2005, 2010; Blum e Chawla, 2001; Blum et al., 2004; Daitch et al., 2009; Liu e Chang, 2009; Melacci e Belkin, 2011; Zhang e Lee, 2007; Zhang e Ando, 2006; Liu et al., 2010; Argyriou et al., 2005; Johnson e Zhang, 2007; Sindhwani et al., 2005; Kong et al., 2012; Ghazvininejad et al., 2011; Zhuang et al., 2012; Nie et al., 2011; Urner et al., 2011; Zhang et al., 2011; Zhang e Zhou, 2009; Tong e Jin, 2007; Wu et al., 2012; Yang et al., 2011; Rigollet, 2007; Lafferty e Wasserman, 2008; Cortes et al., 2008). Dentre os métodos de aprendizado semissupervisionado, os baseados em grafos são amplamente usados na literatura devido ao fato de o grafo ponderado poder aproximar a estrutura de manifold dos dados (Wang e Xia, 2012; Luo et al., 2012), se ela existir.

A comunidade científica propôs uma variedade de algoritmos de aprendizado semissupervisionado baseado em grafos (Belkin et al., 2006; Liu e Chang, 2009; Zhou et al., 2004; Zhu et al., 2003; Liu et al., 2010; Melacci e Belkin, 2011; Zhang et al., 2011) bem como uma variedade de métodos de geração de grafos (Hein e Maier, 2007; Liu e Chang, 2009; Roweis e Saul, 2000; Wang e Xia, 2012; Luo et al., 2012; Zhuang et al., 2012). Apesar de sua crescente popularidade, a literatura de aprendizado semissupervisionado carece de um estudo empírico detalhado que mostre o impacto que os métodos de geração de grafos apresentam tanto no desempenho de classificação quanto na estabilidade dos classificadores semissupervisionados.

Fez-se uma vasta revisão bibliográfica neste trabalho. Entretanto, não foram encontrados trabalhos empíricos capazes de avaliar de maneira efetiva os classificadores semissupervisionados combinados com os métodos de geração de grafos. Tem-se como hipótese deste trabalho que há a possibilidade de se gerar modelos experimentais simples que sejam capazes de avaliar a influência que os métodos de geração de grafos apresentam na classificação 
semissupervisionada. Assim, o objetivo deste trabalho é gerar modelos experimentais capazes de auxiliar o processo de análise experimental e, com isso, evitar a geração de conclusões tendenciosas.

Neste trabalho, foram propostos três modelos de avaliação experimental que visam auxiliar o processo de análise dos resultados obtidos. Apesar de os modelos propostos neste trabalho serem usados na área de aprendizado semissupervisionado, eles podem ser usados também em outras áreas do aprendizado de máquina, como agrupamento espectral e redução de dimensionalidade em grafos. O modelo de avaliação baseado em estabilidade de classificadores pode ser usado tanto na área de aprendizado supervisionado quanto não supervisionado. Para o caso não supervisionado, avalia-se a estabilidade dos métodos de agrupamento.

Este capítulo está organizado como a seguir. Na Seção 1.1, discutem-se as contribuições deste trabalho. Na Seção 1.2, faz-se uma breve discussão sobre os resultados obtidos neste trabalho. Na Seção 1.3, discutem-se as consequências dos resultados obtidos neste trabalho. Por fim, na Seção 1.4, descreve-se a organização deste trabalho.

\subsection{Contribuições}

Neste trabalho, faz-se uma avaliação empírica detalhada que compara algoritmos do estado da arte da área de aprendizado semissupervisionado baseado em grafos combinados com uma variedade de métodos de geração de grafos, incluindo tanto métodos de geração de grafos de adjacência quanto de geração de matrizes ponderadas. A avaliação empírica proposta neste trabalho é subdivida em quatro partes, como descrito a seguir:

Análise de melhor caso. Avaliam-se as melhores taxas de erro de cada combinação de classificador semissupervisionado e método de geração de grafos usando uma variedade de valores para o parâmetro de esparsificação, o qual está relacionado com o número de vizinhos de cada exemplo de treinamento. Apesar de essa abordagem ser a mais usada para comparar classificadores semissupervisionados na literatura (Chapelle et al., 2006; Zhu, 2005; Wu et al., 2012; Wang e Xia, 2012; Luo et al., 2012; Zhuang et al., 2012), esta avaliação empírica por si pode não prover toda a informação necessária para escolher os melhores classificadores para aplicações reais. Como exemplo, classificadores que apresentam elevada estabilidade podem ser preferíveis em relação a classificadores que apresentam excelente desempenho de classificação para um pequeno subconjunto dos valores dos parâmetros e um desempenho bem inferior para os demais valores;

Avaliação da estabilidade dos classificadores. Avalia-se a estabilidade dos classificadores combinados com os métodos de geração de grafos, variando-se o valor do parâmetro de esparsificação. Conforme mencionado anteriormente, esta análise é de fundamental importância dado que um 
classificador pode obter excelentes desempenhos de classificação apenas para uma quantidade restrita de valores dos parâmetros. Acredita-se que esta análise seja uma ferramenta inestimável para identificar quais classificadores proveem um bom balanço entre desempenho de classificação e estabilidade;

Avaliação do impacto da geração de grafos. Avalia-se o comportamento dos métodos de geração de grafos combinados com os classificadores semissupervisionados, variando-se o valor do parâmetro de esparsificação. Nesta análise, busca-se verificar (1) como os métodos de geração de grafos afetam o desempenho de classificação de cada classificador em cada base de dados e (2) a estabilidade dos métodos de geração de grafos em cada base de dados. Para os classificadores que apresentam ao menos um parâmetro de regularização, foram fixados os valores dos parâmetros de regularização com os valores que obtiveram a menor taxa de erro médio na análise de melhor caso e então variou-se o valor do parâmetro de esparsificação;

Avaliação da influência dos parâmetros de regularização. Avaliam-se as superfícies de erro geradas pelos classificadores semissupervisionados que apresentam ao menos um parâmetro de regularização. Para tanto, escolhese o valor do parâmetro de esparsificação que obteve a menor taxa de erro médio na análise de melhor caso e então variam-se os valores dos parâmetros de regularização dos classificadores semissupervisionados para gerar as superfícies de erro.

\subsection{Discussão dos resultados obtidos}

Nesta seção, faz-se uma breve discussão sobre os resultados obtidos neste trabalho. Análises detalhadas dos resultados obtidos encontram-se nos Capítulos 4, 5, 6 e 7 .

Os resultados obtidos neste trabalho mostram que o grafo $k$-vizinhos mais próximos mútuo (mutKNN) pode ser a melhor opção para a geração de grafos de adjacência enquanto que o kernel RBF pode ser a melhor opção para a geração de matrizes ponderadas. Em adição, o grafo mutKNN tende a gerar superfícies de erro que são mais suaves que aquelas geradas pelos outros métodos de geração de grafos de adjacência. Entretanto, o grafo mutKNN é instável para valores relativamente pequenos de $k$.

O algoritmo Transdução Robusta Multi-classe em Grafos (RMGT) (Liu e Chang, 2009) apresentou excelente desempenho de classificação na maioria das bases de dados para todos os métodos de geração de grafos. Entretanto, esse algoritmo não obteve resultados satisfatórios na base de dados que apresentava desbalanceamento de classes. Ainda mais, o algoritmo RMGT apresentou elevada instabilidade na base de dados de classificação de imagens para valores relativamente pequenos de $k$. O algoritmo Máquina de Vetores de Suporte Laplaciana (LapSVM) (Belkin et al., 2006) apresentou excelentes desempenho de 
classificação e estabilidade nas bases de dados de misturas gaussianas para todos os métodos de geração de grafos.

Os resultados para alguns classificadores nas bases de misturas gaussianas são melhores ao se usar grafos densos. Isso indica que a premissa de que grafos esparsos tendem a gerar melhores resultados que grafos densos, amplamente usada na literatura de aprendizado semissupervisionado (Zhu, 2005), pode não ser sempre válida.

Ao se avaliar a estabilidade dos classificadores semissupervisionados, observa-se que há várias trocas de rankings dos classificadores, o que pode dar margem à elaboração de trabalhos empíricos tendenciosos. Uma discussão sobre esse assunto é feita no Capítulo 5. Ao se avaliar a estabilidade dos métodos de geração de grafos, pode-se observar que há várias trocas nos rankings dos grafos, o que também pode dar margem à elaboração de trabalhos empíricos tendenciosos. Uma discussão sobre esse assunto é feita no Capítulo 6.

A partir da análise dos resultados, percebe-se que o desempenho de classificação dos classificadores semissupervisionados é altamente influenciado pelos parâmetros internos (como os parâmetros de regularização) e os parâmetros externos (como o parâmetro de esparsificação). Tal variabilidade não mostrou um padrão evidente que possa ser explorado para auxiliar o processo de escolha de parâmetros. Em adição, a premissa do aprendizado semissupervisionado, a qual assume que haja um conjunto pequeno de exemplos rotulados iniciais, pode inviabilizar o uso de técnicas de estimativa de parâmetro comumente usadas na literatura de aprendizado supervisionado.

\subsection{Consequências dos resultados obtidos}

Os resultados obtidos apresentam duas consequências principais:

Para os usuários. Dada uma base de dados, é difícil recomendar um classificador semissupervisionado, um valor para o parâmetro de esparsificação e/ou os valores para os parâmetros de regularização que poderiam obter um bom desempenho de classificação. Como a quantidade de exemplos rotulados é bem restrita em aplicações de aprendizado semissupervisionado, o usuário não possui nenhuma ferramenta para informá-lo como escolher uma boa configuração de parâmetros tendo em vista que a maioria dos métodos de seleção de modelos atua de maneira supervisionada. Como será mostrado neste trabalho, a escolha incorreta dos valores dos parâmetros pode afetar seriamente o desempenho de classificação dos algoritmos usados;

Para os pesquisadores. Trocas nos valores dos parâmetros podem causar trocas nos rankings relativos entre os classificadores. Isso significa que, para uma base de dados específica, vários métodos podem ser considerados como o melhor classificador para um dado subconjunto dos valores dos parâmetros. Isso é um problema sério pois a evidência empírica de 
que um método é superior aos outros pode ser apenas confirmada para um conjunto restrito dos valores dos parâmetros. Em adição, essa variabilidade do desempenho de classificação pode dificultar a reprodução dos resultados experimentais de artigos que não reportam claramente cada valor de parâmetro usado na avaliação experimental.

\subsection{Organização do Trabalho}

Este trabalho está organizado como a seguir. No Capítulo 2, apresenta-se a revisão bibliográfica referente à classificação semissupervisionada baseada em grafos, tendo como foco o estudo de métodos de geração de grafos a partir de uma amostra de exemplos descrita no espaço vetorial $\mathbb{R}^{d}$ e os métodos de classificação mais usados na literatura. No Capítulo 3, descreve-se o protocolo experimental usado neste trabalho. No Capítulo 4, comparam-se os classificadores semissupervisionados combinados com os métodos de geração de grafos usando a análise de melhor caso. No Capítulo 5, avalia-se a estabilidade dos classificadores semissupervisionados combinados com os métodos de geração de grafos. No Capítulo 6, avalia-se a estabilidade dos métodos de geração de grafos combinados com os classificadores semissupervisionados. No Capítulo 7, avalia-se a influência dos parâmetros de regularização no desempenho de classificação dos algoritmos semissupervisionados. Por fim, no Capítulo 8, descrevem-se as conclusões deste trabalho e sugerem-se direções para trabalhos futuros. 



\section{CAPÍtUlo 2 \\ Classificação semissupervisionada baseada em grafos}

Neste capítulo, faz-se uma revisão da literatura de aprendizado semissupervisionado, abordando os métodos de geração de grafos e os classificadores semissupervisionados usados na avaliação empírica deste trabalho. Faz-se uma revisão bibliográfica em profundidade tendo como foco os métodos de geração de grafos e de classificação semissupervisionada baseada em grafos mais usados na literatura (Zhu, 2005; Chapelle et al., 2006; Kong et al., 2012; Wang e Xia, 2012; Luo et al., 2012; Ghazvininejad et al., 2011; Zhuang et al., 2012; Nie et al., 2011; Urner et al., 2011; Zhang et al., 2011; Wu et al., 2012; Yang et al., 2011).

Este capítulo está organizado da seguinte maneira. Na Seção 2.1, discutemse os conceitos preliminares da área de aprendizado semissupervisionado baseado em grafos adotados neste trabalho. Na Seção 2.2, descrevem-se os métodos mais usados para se gerar grafos de adjacência. Na Seção 2.3, descrevem-se os métodos mais usados para se gerar matrizes ponderadas. Na Seção 2.4, descrevem-se os principais classificadores semissupervisionados baseados em grafos do estado da arte. Na Seção 2.5, discute-se o processo de indução heurística a partir de modelos transdutivos.

\subsection{Preliminares}

Considere $\mathcal{X}:=\left\{\boldsymbol{x}_{i}\right\}_{i=1}^{n} \subset \mathbb{R}^{d}$ uma amostra de exemplos de treinamento. Sem perda de generalidade, considere que os $l$ primeiros exemplos em $\mathcal{X}$ sejam rotulados. O exemplo $\boldsymbol{x}_{i}(i \leq l)$ está associado a um rótulo $y_{i} \in \mathbb{N}_{c}$ tal que $\mathbb{N}_{p}:=\left\{i \in \mathbb{N}^{*} \mid 1 \leq i \leq p\right\}$, com $p \in \mathbb{N}^{*}$, e $c$ representa o número de classes. $\mathrm{O}$ símbolo $:=$ é usado neste trabalho para indicar igualdade por definição. Considere $\mathbb{B}:=\{0,1\}$. Seja $u:=n-l$ a quantidade de exemplos não rotulados e $\boldsymbol{Y} \in \mathbb{B}^{n \times c}$ a matriz de rótulos em que $\boldsymbol{Y}_{i j}=1$ se, e somente se, $\boldsymbol{x}_{i}$ estiver 
associado ao rótulo $y_{i}=j$. Matematicamente,

$$
\mathbf{Y}_{i j}=\left\{\begin{array}{l}
1, \quad i \leq l \wedge y_{i}=j \\
0, \quad \text { caso contrário }
\end{array}\right.
$$

Considere um grafo esparso não orientado $\mathcal{G}:=(\mathcal{X}, \mathcal{E})$ em que cada exemplo $\boldsymbol{x}_{i}$ seja um vértice de $\mathcal{G} . \mathcal{E} \subseteq \mathcal{X} \times \mathcal{X}$ representa o conjunto de arestas de $\mathcal{G}$. Neste trabalho, considera-se apenas o uso de grafos não orientados, tendo em vista que eles são os mais comumente usados na literatura ( $\mathrm{Zhu}, 2005$; Chapelle et al., 2006; Zhuang et al., 2012; Wang e Xia, 2012; Luo et al., 2012; Urner et al., 2011; Zhang et al., 2011; Wu et al., 2012; Yang et al., 2011). Seja $\mathcal{N}_{i} \subset \mathcal{X}$ o conjunto de vizinhos do exemplo $\boldsymbol{x}_{i}$ e $\boldsymbol{x}_{i_{k}} \in \mathbb{R}^{d}$ o $k$-ésimo vizinho do exemplo $\boldsymbol{x}_{i}$. Para se gerar uma matriz de pesos $\boldsymbol{W} \in \mathbb{R}^{n \times n}$ a partir de $\mathcal{G}$, usa-se uma função de similaridade $\mathcal{K}: \mathbb{R}^{d} \times \mathbb{R}^{d} \rightarrow \mathbb{R}$ para computar os pesos das arestas entre cada exemplo $\boldsymbol{x}_{i}$ e seus vizinhos $\boldsymbol{x}_{j} \in \mathcal{N}_{i}$.

Definem-se a seguir alguns dos conceitos usados no decorrer deste trabalho. Uma matriz simétrica $\boldsymbol{A} \in \mathbb{R}^{n \times n}$ é semi-definida positiva se $\boldsymbol{v}^{\top} \boldsymbol{A} \boldsymbol{v} \geq 0$, $\forall \boldsymbol{v} \in \mathbb{R}^{n}$. Escreve-se $\boldsymbol{A} \succcurlyeq 0$ para indicar que $\boldsymbol{A}$ é semi-definida positiva. O símbolo $^{\top}$ indica a transposição de matrizes ou vetores. O operador $\operatorname{diag}(\cdot)$ recebe como entrada um vetor $\boldsymbol{v} \in \mathbb{R}^{a}$, com $a \in \mathbb{N}^{*}$, e retorna uma matriz diagonal $\boldsymbol{V} \in \mathbb{R}^{a \times a}$ tal que $\boldsymbol{V}_{i i}=\boldsymbol{v}_{i}, \forall i \in \mathbb{N}_{a}$. $\boldsymbol{I}_{n}$ representa a matriz identidade de ordem $n$.

Um modelo matricial de suma importância para o aprendizado em grafos é o Laplaciano do grafo, que é um operador que gera uma matriz semi-definida positiva a partir de uma dada matriz simétrica não negativa. O Laplaciano combinatório é definido por:

$$
\Delta:=\boldsymbol{D}-\boldsymbol{W}
$$

em que $\boldsymbol{D}:=\operatorname{diag}\left(\boldsymbol{W} \mathbf{1}_{n}\right)$ tal que $\boldsymbol{1}_{n}$ é um vetor $n$-dimensional cujas entradas são todas 1. Logo:

$$
\boldsymbol{\Delta}_{i j}=\left\{\begin{array}{rr}
\boldsymbol{D}_{i i}, & i=j \\
-\boldsymbol{W}_{i j}, & i \neq j
\end{array}\right.
$$

O Laplaciano normalizado é definido por:

$$
\boldsymbol{L}:=\boldsymbol{D}^{-1 / 2} \boldsymbol{\Delta} \boldsymbol{D}^{-1 / 2}=\boldsymbol{I}_{n}-\boldsymbol{D}^{-1 / 2} \boldsymbol{W} \boldsymbol{D}^{-1 / 2}
$$

Logo:

$$
\boldsymbol{L}_{i j}=\left\{\begin{aligned}
1, & i=j \\
-\frac{\boldsymbol{W}_{i j}}{\sqrt{\boldsymbol{D}_{i i} \boldsymbol{D}_{j j}}}, & i \neq j
\end{aligned}\right.
$$

Para um dado vetor $\boldsymbol{f} \in \mathbb{R}^{n}$, pode-se escrever: 


$$
\begin{aligned}
\boldsymbol{f}^{\top} \boldsymbol{\Delta} \boldsymbol{f} & =\frac{1}{2} \sum_{i=1}^{n} \sum_{j=1}^{n} \boldsymbol{W}_{i j}\left(\boldsymbol{f}_{i}-\boldsymbol{f}_{j}\right)^{2} \\
\boldsymbol{f}^{\top} \boldsymbol{L} \boldsymbol{f} & =\frac{1}{2} \sum_{i=1}^{n} \sum_{j=1}^{n} \boldsymbol{W}_{i j}\left(\frac{1}{\sqrt{\boldsymbol{D}_{i i}}} \boldsymbol{f}_{i}-\frac{1}{\sqrt{\boldsymbol{D}_{j j}}} \boldsymbol{f}_{j}\right)^{2}
\end{aligned}
$$

As Equações (2.1) e (2.2) mostram que $\Delta$ e $\boldsymbol{L}$ são semi-definidas positivas $(\boldsymbol{\Delta} \succcurlyeq 0$ e $\boldsymbol{L} \succcurlyeq 0)$ para uma dada matriz simétrica não negativa $\boldsymbol{W}$. Para garantir que os Laplacianos do grafo sejam matrizes positivas semi-definidas, impõemse as seguintes restrições na matriz de pesos $\boldsymbol{W}$ :

Tal propriedade é bastante interessante para o aprendizado em grafos, tendo em vista que o modelo de otimização de vários algoritmos passa a ser convexo. Isso remove o problema de haver diversos mínimos (ou máximos) locais na superfície de erro e a solução global desses algoritmos passa a ser encontrada de maneira analítica.

Para uma formulação geral de operadores Laplacianos em grafos, veja o trabalho de Hein et al. (2007). Para garantir que os Laplacianos do grafo sejam matrizes semi-definidas positivas, impõem-se as seguintes restrições na matriz de pesos $\boldsymbol{W}$ :

$$
\boldsymbol{W}=\boldsymbol{W}^{\top}, \quad \boldsymbol{W} \geq 0, \quad \operatorname{tr}(\boldsymbol{W})=0
$$

Usa-se a notação $\boldsymbol{W} \geq 0$ para indicar que a matriz $\boldsymbol{W}$ é não negativa. Denota-se o traço de uma matriz $\boldsymbol{M} \in \mathbb{R}^{a \times a}$ por $\operatorname{tr}(\boldsymbol{M}):=\sum_{i=1}^{a} \boldsymbol{M}_{i i}$. Assumese que todas as matrizes sejam subdivididas em submatrizes de exemplos rotulados e não rotulados. Seja $\boldsymbol{F} \in \mathbb{R}^{n \times c}$ a matriz de saída de um dado classificador. Por definição, considera-se que $\boldsymbol{F}$ e $\boldsymbol{Y}$ sejam subdivididas em duas submatrizes enquanto que todas as outras matrizes sejam subdivididas em quatro submatrizes. Por exemplo:

$$
\boldsymbol{W}=\left[\begin{array}{ll}
\boldsymbol{W}_{\mathcal{L L}} & \boldsymbol{W}_{\mathcal{L U}} \\
\boldsymbol{W}_{\mathcal{U L}} & \boldsymbol{W}_{\mathcal{U U}}
\end{array}\right] \quad \mathbf{Y}=\left[\begin{array}{l}
\mathbf{Y}_{\mathcal{L}} \\
\mathbf{Y}_{\mathcal{U}}
\end{array}\right]
$$

$\boldsymbol{W}_{\mathcal{L L}} \in \mathbb{R}^{l \times l}$ e $\boldsymbol{Y}_{\mathcal{L}} \in \mathbb{B}^{l \times c}$ são submatrizes de $\boldsymbol{W}$ e $\boldsymbol{Y}$, respectivamente, para os exemplos rotulados, e assim por diante. Por definição, $\boldsymbol{Y}_{\mathcal{L}} \mathbf{1}_{c}=\mathbf{1}_{l}$ e $\boldsymbol{Y}_{\mathcal{U}}=\boldsymbol{O}_{u \times c}$ tal que $\boldsymbol{O}_{u \times c}$ é uma matriz nula de tamanho $u \times c$.

Uma etapa crucial para o aprendizado semissupervisionado baseado em grafos é o processo de geração de grafos (Zhu, 2005). Tal processo pode ser dividido em duas etapas: (1) geração do grafo de adjacência, na qual gera-se um grafo esparso não orientado $\mathcal{G}$ a partir do conjunto de treinamento $\mathcal{X}$. Essa etapa é descrita na Seção 2.2; (2) geração de matrizes ponderadas, a qual gera uma matriz de pesos simétrica não negativa $\boldsymbol{W}$ a partir de um grafo esparso $\mathcal{G}$ usando uma função de similaridade $\mathcal{K}$. Tal etapa é descrita na Seção 2.3. 


\subsection{Geração de grafos de adjacência}

A etapa de geração de grafos de adjacência visa gerar um grafo esparso não orientado $\mathcal{G}$ a partir do conjunto de treinamento $\mathcal{X}$ usando uma função de distância $\Psi: \mathbb{R}^{d} \times \mathbb{R}^{d} \rightarrow \mathbb{R}$. Seja $\Psi \in \mathbb{R}^{n \times n}$ uma matriz de distâncias, tal que $\boldsymbol{\Psi}_{i j}:=\Psi\left(\boldsymbol{x}_{i}, \boldsymbol{x}_{j}\right), \forall i, j \in \mathbb{N}_{n}$, e $\boldsymbol{A} \in \mathbb{B}^{n \times n}$ uma matriz de adjacência em que $\boldsymbol{A}_{i j}=1$ se, e somente se, $\boldsymbol{x}_{j} \in \mathcal{N}_{i}, \forall i, j \in \mathbb{N}_{n}$. São descritas a seguir as maneiras mais usadas para se gerar grafos de adjacência a partir de $\mathcal{X}$.

Grafo $\epsilon$-vizinhaça No grafo $\epsilon$-vizinhaça, há uma aresta entre os exemplos $\boldsymbol{x}_{i}$ e $\boldsymbol{x}_{j}$ se, e somente se, $\Psi\left(\boldsymbol{x}_{i}, \boldsymbol{x}_{j}\right) \leq \epsilon$ tal que $\epsilon \in \mathbb{R}_{+}^{*}$ é um parâmetro livre. Em geral, grafos $\epsilon$-vizinhança não são amplamente usados em situações práticas devido ao fato de o mesmo poder conter diversos componentes que violem a Premissa 1 (ver pág. 12). Por esse motivo, grafos $\epsilon$-vizinhança não serão usados na avaliação experimental deste trabalho.

Considerando que $\mathcal{H}(\cdot)$ seja a função de Heaviside, gera-se a matriz de adjacência do grafo $\epsilon$-vizinhança por $\boldsymbol{A}_{i j}=\mathcal{H}\left(\epsilon-\Psi\left(\boldsymbol{x}_{i}, \boldsymbol{x}_{j}\right)\right), \forall i, j \in \mathbb{N}_{n}$. A função $\mathcal{H}$ é definida por:

$$
\mathcal{H}(x)= \begin{cases}1, & x \geq 0 \\ 0, & x<0\end{cases}
$$

Usando $\mathcal{H}(\cdot)$, gera-se a matriz de adjacência do grafo $\epsilon$-vizinhança por

$$
\boldsymbol{A}_{i j}=\mathcal{H}\left(\epsilon-\Psi\left(\boldsymbol{x}_{i}, \boldsymbol{x}_{j}\right)\right)
$$

Grafo $k$-vizinhos mais próximos $(k \mathbf{N N}) \quad$ No grafo $k \mathrm{NN}$, há uma aresta orientada entre $\boldsymbol{x}_{i}$ e $\boldsymbol{x}_{j}$ se $\boldsymbol{x}_{j}$ é um dos $k$ vizinhos mais próximos de $\boldsymbol{x}_{i}$. Matematicamente, para computar a matriz de adjacência do grafo $k \mathrm{NN}$, resolve-se o seguinte problema de otimização:

$$
\begin{gathered}
\min _{\widehat{\boldsymbol{A}} \in \mathbb{B}^{n \times n}} \operatorname{tr}\left(\widehat{\boldsymbol{A}}^{\top} \Psi\right)=\sum_{i=1}^{n} \sum_{j=1}^{n} \widehat{\boldsymbol{A}}_{i j} \Psi\left(\boldsymbol{x}_{i}, \boldsymbol{x}_{j}\right) \\
\text { s.a. } \widehat{\boldsymbol{A}} \mathbf{1}_{n}=k \mathbf{1}_{n}, \operatorname{tr}(\widehat{\boldsymbol{A}})=0
\end{gathered}
$$

O problema de otimização em (2.5) pode ser resolvido de maneira gulosa escolhendo-se os $k$ vizinhos mais próximos de cada exemplo do conjunto de treinamento $\mathcal{X}$. Em adição, o problema de otimização em (2.5) pode ser facilmente reformulado para o problema de se gerar um grafo $\epsilon$ vizinhança usando um valor de $\epsilon$ adaptativo referente a cada exemplo $\boldsymbol{x}_{i}$. Considerando $\epsilon_{i}:=\Psi\left(\boldsymbol{x}_{i}, \boldsymbol{x}_{i_{k}}\right)$, pode-se computar a matriz de adjacência do grafo $k \mathrm{NN}$ de tal forma que

$$
\boldsymbol{A}_{i j}=\mathcal{H}\left(\epsilon_{i}-\Psi\left(\boldsymbol{x}_{i}, \boldsymbol{x}_{j}\right)\right),
$$


$\forall i, j \in \mathbb{N}_{n}$. Como a matriz de adjacência do grafo $k \mathrm{NN}$ pode não ser simétrica, uma etapa de pós-processamento faz-se necessária. Para tanto, três métodos foram criados para simetrizar a matriz de adjacência do grafo $k \mathrm{NN}$ : $k \mathrm{NN}$ mútuo $\left(m u t K N N^{1}\right.$ ), o qual gera uma matriz de adjacência

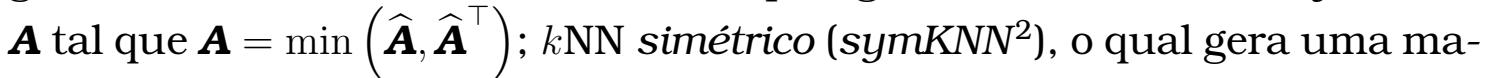
triz de adjacência $\boldsymbol{A}$ tal que $\boldsymbol{A}=\max \left(\widehat{\boldsymbol{A}}, \widehat{\boldsymbol{A}}^{\top}\right)$; e $k \mathrm{NN}$ favorável à simetria $\left(\right.$ symFKNN ${ }^{3}$ ) (Liu e Chang, 2009), o qual gera uma matriz de adjacência não binária $\boldsymbol{A}$ tal que $\boldsymbol{A}=\widehat{\boldsymbol{A}}+\widehat{\boldsymbol{A}}^{\top}$. As variações simétrica e mútua do grafo $k \mathrm{NN}$ foram amplamente estudadas na área de agrupamento em grafos (Maier et al., 2007, 2009a), apresentando um impacto elevado em medidas de avaliação de modelos de agrupamento baseados em grafos (Maier et al., 2009b).

\subsection{Geração de matrizes ponderadas}

Dada uma matriz de adjacência $\boldsymbol{A}$, gera-se uma matriz de pesos $\boldsymbol{W} \in \mathbb{R}^{n \times n}$ usando uma função de similaridade $\mathcal{K}: \mathbb{R}^{d} \times \mathbb{R}^{d} \rightarrow \mathbb{R}$ para computar os valores $\boldsymbol{W}_{i j}$ em que $\boldsymbol{x}_{j} \in \mathcal{N}_{i}$. As técnicas mais usadas para se gerar matrizes ponderadas são descritas a seguir. Duas dessas técnicas, o kernel RBF e a similaridade de Hein e Maier (2007), geram $\boldsymbol{W}$ de tal forma que $\boldsymbol{W}_{i j}=\boldsymbol{A}_{i j} \mathcal{K}\left(\boldsymbol{x}_{i}, \boldsymbol{x}_{j}\right)$. Modelos baseados em reconstrução linear (Roweis e Saul, 2000) geram $\boldsymbol{W}$ sem usar uma função de similaridade explicitamente.

Kernel RBF O kernel $\mathrm{RBF}^{4}$ (ou Gaussiano) computa a similaridade entre os exemplos $\boldsymbol{x}_{i}$ e $\boldsymbol{x}_{j}$ como a seguir:

$$
\mathcal{K}\left(\boldsymbol{x}_{i}, \boldsymbol{x}_{j}\right)=\exp \left(-\frac{\Psi^{2}\left(\boldsymbol{x}_{i}, \boldsymbol{x}_{j}\right)}{2 \sigma^{2}}\right)
$$

em que $\sigma \in \mathbb{R}_{+}^{*}$ é um parâmetro livre.

Similaridade de Hein e Maier (2007) (HM) Seja $\psi: \mathcal{X} \times \mathbb{N}^{*} \mapsto \mathbb{R}$ uma função em que $\psi\left(\boldsymbol{x}_{i}, k\right):=\Psi\left(\boldsymbol{x}_{i}, \boldsymbol{x}_{i_{k}}\right)$. Com isso, define-se a similaridade HM por:

$$
\mathcal{K}\left(\boldsymbol{x}_{i}, \boldsymbol{x}_{j}\right)=\exp \left(-\frac{\Psi^{2}\left(\boldsymbol{x}_{i}, \boldsymbol{x}_{j}\right)}{\left(\max \left\{\psi\left(\boldsymbol{x}_{i}, k\right), \psi\left(\boldsymbol{x}_{j}, k\right)\right\}\right)^{2}}\right)
$$

A similaridade HM é basicamente um kernel RBF com uma abertura de kernel adaptativa.

\footnotetext{
${ }^{1}$ Mutual kNN.

${ }^{2}$ Symmetric kNN.

${ }^{3}$ Symmetry favored $k N N$.

${ }^{4}$ Radial Basis Function.
} 
Local Linear Embedding (LLE) A abordagem LLE (Roweis e Saul, 2000; Saul e Roweis, 2003) gera uma matriz de pesos $\boldsymbol{W}$ resolvendo o seguinte problema de otimização:

$$
\min _{\mathbf{W} \in \mathbb{R}^{n \times n}} \sum_{i=1}^{n}\left\|\boldsymbol{x}_{i}-\sum_{\boldsymbol{x}_{j} \in \mathcal{N}_{i}} \boldsymbol{W}_{i j} \boldsymbol{x}_{j}\right\|_{2}^{2} \text { s.a. } \quad \boldsymbol{W} \mathbf{1}_{n}=\mathbf{1}_{n}, \quad \boldsymbol{W} \geq 0
$$

O símbolo $\|\cdot\|_{2}$ representa a norma $l_{2}$.

\subsection{Classificadores semissupervisionados}

Dada uma matriz de pesos $\boldsymbol{W}$, os classificadores semissupervisionados baseados em grafos usam $\boldsymbol{W}$ e a matriz de rótulos $\mathbf{Y}$ para gerar uma matriz de saída $\boldsymbol{F}$ por meio de difusão de rótulos no grafo ponderado ou regularização em $\boldsymbol{W}$. Apesar de seu aparente sucesso empírico, há uma condição intrínseca para se aplicar modelos de aprendizado semissupervisionado baseado em grafos para classificar todos os exemplos não rotulados em $\mathcal{X}$. A Premissa 1 descreve essa condição, a qual, infelizmente, não é explicitada na literatura.

Premissa 1 Cada exemplo não rotulado está em um subgrafo conexo em que há pelo menos um exemplo rotulado, ou seja, para cada exemplo não rotulado, há um caminho entre este e um exemplo rotulado qualquer.

Como a maioria dos métodos de geração de grafos ignora a informação dos rótulos iniciais, os grafos gerados por tais métodos podem não satisfazer a Premissa 1. Com isso, alguns exemplos poderiam não ser classificados, acarretando uma diminuição no desempenho de classificação dos algoritmos de aprendizado semissupervisionado.

A seguir, faz-se uma revisão dos classificadores semissupervisionados baseados em grafos do estado da arte.

Campos Aleatórios Gaussianos (GRF) O algoritmo GRF ${ }^{5}$ (Zhu et al., 2003) resolve o seguinte problema de otimização:

$$
\min _{\boldsymbol{F} \in \mathbb{R}^{n \times c}} \operatorname{tr}\left(\boldsymbol{F}^{\top} \boldsymbol{\Delta} \boldsymbol{F}\right) \quad \text { s.a. } \quad \boldsymbol{F}_{\mathcal{L}}=\boldsymbol{Y}_{\mathcal{L}}
$$

Chega-se à seguinte solução global:

$$
\boldsymbol{F}_{\mathcal{U}}=-\Delta_{\mathcal{U} \mathcal{U}}^{-1} \Delta_{\mathcal{U L}} \boldsymbol{Y}_{\mathcal{L}}
$$

\footnotetext{
${ }^{5}$ Gaussian Random Fields.
} 
Consistência Local e Global (LGC) O algoritmo LGC $^{6}$ (Zhou et al., 2004) resolve o seguinte problema de otimização:

$$
\min _{\boldsymbol{F} \in \mathbb{R}^{n \times c}} \operatorname{tr}\left(\boldsymbol{F}^{\top} \boldsymbol{L} \boldsymbol{F}+\mu(\boldsymbol{F}-\boldsymbol{Y})^{\top}(\boldsymbol{F}-\boldsymbol{Y})\right)
$$

Chega-se à solução global:

$$
\boldsymbol{F}=\left(\boldsymbol{I}_{n}+\frac{1}{\mu} \boldsymbol{L}\right)^{-1} \boldsymbol{Y}
$$

Guadrados Mínimos Regularizados Laplacianos (LapRLS) O algoritmo LapRLS$^{7}$ (Belkin et al., 2006) resolve o seguinte problema de otimização:

$$
\min _{f \in \mathcal{H}_{\mathcal{K}}} \frac{1}{l} \sum_{i=1}^{l}\left(y_{i}-f\left(\boldsymbol{x}_{i}\right)\right)^{2}+\gamma_{A}\|f\|_{\mathcal{H}_{\mathcal{K}}}+\gamma_{I} \boldsymbol{f}^{\top} \boldsymbol{\Delta} \boldsymbol{f}
$$

em que $\mathcal{H}_{\mathcal{K}}$ é o espaço de Hilbert de kernel de reprodução $\left(R_{K H} S^{8}\right)$ correspondente ao kernel $\mathcal{K} ;\|\cdot\|_{\mathcal{H}_{\mathcal{K}}}$ é a norma em $\mathcal{H}_{\mathcal{K}} ; \boldsymbol{f}:=\left[f\left(\boldsymbol{x}_{1}\right) \cdots f\left(\boldsymbol{x}_{n}\right)\right]^{\top} \in \mathbb{R}^{n}$ tal que $f: \mathcal{X} \mapsto \mathbb{R} ; \gamma_{A}$ e $\gamma_{I}$ são parâmetros de regularização. Devido ao Teorema Representativo de Belkin et al. (2006), a solução do problema de otimização em (2.11) pode ser expressa por meio de expansão de kernel usando tanto exemplos rotulados quanto não rotulados. Matematicamente:

$$
f(\boldsymbol{x})=\sum_{i=1}^{n} \mathcal{K}\left(\boldsymbol{x}, \boldsymbol{x}_{i}\right) \boldsymbol{\alpha}_{i} \Rightarrow \boldsymbol{f}=\mathbf{K} \boldsymbol{\alpha}
$$

Ao efetuar a expansão de kernel $\boldsymbol{f}=\boldsymbol{K} \boldsymbol{\alpha}$, chega-se à seguinte solução:

$$
\boldsymbol{\alpha}=\left(\boldsymbol{J K}+\gamma_{A} l \mathbf{I}_{n}+\gamma_{I} l \Delta \boldsymbol{K}\right)^{-1} \boldsymbol{y}
$$

em que

$$
\boldsymbol{y}:=\left[y_{1}, \cdots, y_{l}, 0, \cdots, 0\right]^{\top} \in \mathbb{R}^{n}
$$

é o vetor de rótulos inicial cujos últimos $u$ valores são zero e

$$
\boldsymbol{J}:=\operatorname{diag}\left([1, \cdots, 1,0, \cdots, 0]^{\top}\right) \in \mathbb{R}^{n \times n}
$$

tal que os $l$ primeiros valores da diagonal de $\boldsymbol{J}$ são iguais a 1 e o restante é 0 . Considera-se que $y_{i} \in\{-1,+1\}, \forall i \in \mathbb{N}_{l}$.

\footnotetext{
${ }^{6}$ Local and Global Consistency.

${ }^{7}$ Laplacian Regularized Least Squares.

${ }^{8}$ Reproducing Kernel Hilbert Space.
} 
Máquinas de Vetores de Suporte Laplacianas (LapSVM) O algoritmo Lap$\mathrm{SVM}^{9}$ (Belkin et al., 2006) resolve o seguinte problema de otimização:

$$
\min _{f \in \mathcal{H}_{\mathcal{K}}} \frac{1}{l} \sum_{i=1}^{l} \max \left(0,1-y_{i} f\left(\boldsymbol{x}_{i}\right)\right)+\gamma_{A}\|f\|_{K}+\gamma_{I} \boldsymbol{f}^{\top} \boldsymbol{\Delta} \boldsymbol{f}
$$

Resolvendo (2.14), obtêm-se a seguinte solução:

$$
\boldsymbol{\alpha}=\frac{1}{2}\left(\gamma_{A} \boldsymbol{I}_{n}+\gamma_{I} \boldsymbol{\Delta} \boldsymbol{K}\right)^{-1} \overline{\boldsymbol{J}}^{\top} \overline{\mathbf{Y}} \boldsymbol{\beta}^{*}
$$

em que $\overline{\boldsymbol{J}}:=\left[\begin{array}{cc}\boldsymbol{I}_{l} & \boldsymbol{O}_{l \times u}\end{array}\right] \in \mathbb{R}^{l \times n}$, tal que $\boldsymbol{O}_{l \times u}$ representa a matriz nula de tamanho $l \times u$ e $\overline{\boldsymbol{Y}}:=\operatorname{diag}\left(\left[y_{1}, \cdots, y_{l}\right]^{\top}\right) \in \mathbb{R}^{l \times l}$. O vetor $\boldsymbol{\beta}^{*} \in \mathbb{R}^{l}$ é a solução do seguinte problema de programação quadrática:

$$
\begin{aligned}
& \boldsymbol{\beta}^{*}=\underset{\boldsymbol{\beta} \in \mathbb{R}^{l}}{\arg \min } \mathbf{1}_{l}^{\top} \boldsymbol{\beta}-\frac{1}{2} \boldsymbol{\beta}^{\top} \boldsymbol{Q} \boldsymbol{\beta} \\
& \text { s.a. } \quad \boldsymbol{y}^{\top} \boldsymbol{\beta}=0, \quad 0 \leq \boldsymbol{\beta} \leq \frac{1}{l}
\end{aligned}
$$

tal que

$$
\boldsymbol{Q}=\frac{1}{2} \overline{\boldsymbol{Y}} \overline{\boldsymbol{J}} \mathbf{K}\left(\gamma_{A} \boldsymbol{I}_{n}+\gamma_{I} \boldsymbol{\Delta} \mathbf{K}\right)^{-1} \overline{\boldsymbol{J}}^{\top} \overline{\boldsymbol{Y}}
$$

Transdução Multi-classe Robusta em Grafos (RMGT) O algoritmo RMGT ${ }^{10}$ (Liu e Chang, 2009) é uma modificação do algoritmo GRF. Assumindo que $\boldsymbol{\omega} \in \mathbb{R}^{c}$ represente as probabilidades a priori de cada classe, o algoritmo RMGT resolve o seguinte problema de otimização:

$$
\min _{\boldsymbol{F} \in \mathbb{R}^{n \times c}} \operatorname{tr}\left(\boldsymbol{F}^{\top} \boldsymbol{\Delta} \boldsymbol{F}\right) \quad \text { s.a. } \quad \boldsymbol{F}_{\mathcal{L}}=\boldsymbol{Y}_{\mathcal{L}}, \quad \boldsymbol{F} \mathbf{1}_{c}=\mathbf{1}_{n}, \quad \boldsymbol{F}^{\top} \mathbf{1}_{n}=n \boldsymbol{\omega}
$$

Chega-se à seguinte solução global:

$$
\boldsymbol{F}_{\mathcal{U}}=-\boldsymbol{\Delta}_{\mathcal{U} \mathcal{U}}^{-1} \boldsymbol{\Delta}_{\mathcal{U} \mathcal{L}} \boldsymbol{Y}_{\mathcal{L}}+\frac{\boldsymbol{\Delta}_{\mathcal{U} \mathcal{U}}^{-1} \mathbf{1}_{u}}{\mathbf{1}_{u}^{\top} \boldsymbol{\Delta}_{\mathcal{U} \mathcal{U}}^{-1} \mathbf{1}_{u}}\left(n \boldsymbol{\omega}^{\top}-\mathbf{1}_{l}^{\top} \boldsymbol{Y}_{\mathcal{L}}+\mathbf{1}_{u}^{\top} \boldsymbol{\Delta}_{\mathcal{U} \mathcal{U}}^{-1} \boldsymbol{\Delta}_{\mathcal{U} \mathcal{L}} \boldsymbol{Y}_{\mathcal{L}}\right)
$$

\footnotetext{
${ }^{9}$ Laplacian Support Vector Machine.

${ }^{10}$ Robust Multi-class Graph Transduction.
} 


\subsection{Indução heurística}

Para realizar o processo de indução a partir de modelos transdutivos, podese realizar uma indução heurística, ou seja, pode-se classificar novos exemplos tendo como base a matriz de saída desses classificadores e a similaridade entre os exemplos de teste e os exemplos de treinamento. O modelo de indução heurística mais usado no aprendizado de máquina é o modelo de regressão não paramétrica de Nadaraya-Watson (Hastie et al., 2009), o qual pode ser visto como uma função $y: \mathbb{R}^{d} \mapsto \mathbb{N}_{c}$ tal que

$$
y(\widetilde{\boldsymbol{x}})=\underset{i \in \mathbb{N}_{c}}{\arg \max } \frac{\sum_{\boldsymbol{x}_{j} \in \widetilde{\mathcal{N}}} \mathcal{K}\left(\widetilde{\boldsymbol{x}}, \boldsymbol{x}_{j}\right) \boldsymbol{F}_{j i}}{\sum_{\boldsymbol{x}_{j} \in \widetilde{\mathcal{N}}} \mathcal{K}\left(\widetilde{\boldsymbol{x}}, \boldsymbol{x}_{j}\right)}=\underset{i \in \mathbb{N}_{c}}{\arg \max } \sum_{\boldsymbol{x}_{j} \in \widetilde{\mathcal{N}}} \mathcal{K}\left(\widetilde{\boldsymbol{x}}, \boldsymbol{x}_{j}\right) \boldsymbol{F}_{j i}
$$

em que $\mathcal{K}(\cdot, \cdot)$ é uma função de similaridade e $\widetilde{\boldsymbol{x}} \in \mathbb{R}^{d}$ é um exemplo qualquer. Com o modelo de Nadaraya-Watson, pode-se realizar indução a partir de qualquer modelo transdutivo. Logo, do ponto de vista experimental, não há métodos puramente transdutivos. Entretanto, do ponto de vista teórico, há poucos métodos semissupervisionados que sejam naturalmente indutivos (Belkin et al., 2006).

Extensão de funções empíricas (ou geração de modelos de indução a partir de modelos transdutivos) é um tópico relevante para o aprendizado de máquina, tendo em vista que haveria a possibilidade de se usar modelos transdutivos em aplicações reais que sejam de natureza indutiva. Bengio et al. (2004) descreveram eficientes modelos de extensão de funções empíricas para diversos modelos de aprendizado em manifolds. Delalleau et al. (2005) propuseram um eficiente modelo de indução para métodos de aprendizado semissupervisionado baseado em grafos tendo como base a regressão não paramétrica de Nadaraya-Watson combinada com uma subamostragem de exemplos usando regularização Laplaciana.

de Sousa (2012) propôs algumas ideias preliminares de como combinar aprendizado em grafos com redes neurais alimentadas adiante $\left(\mathrm{FNN}^{11}\right)$ por meio da inserção dos Laplacianos do grafo no modelo de otimização das FNNs. Os Laplacianos do grafo serviriam para "suavizar" a saída do classificador em regiões de alta densidade. A geração de modelos indutivos de maneira eficiente usando FNNs se deve ao fato da não necessidade de se armazenar o conjunto de treinamento para se realizar indução, diferentemente do que ocorre nos modelos indutivos baseados em grafos (Belkin et al., 2006).

\footnotetext{
${ }^{11}$ Feedforward neural networks.
} 



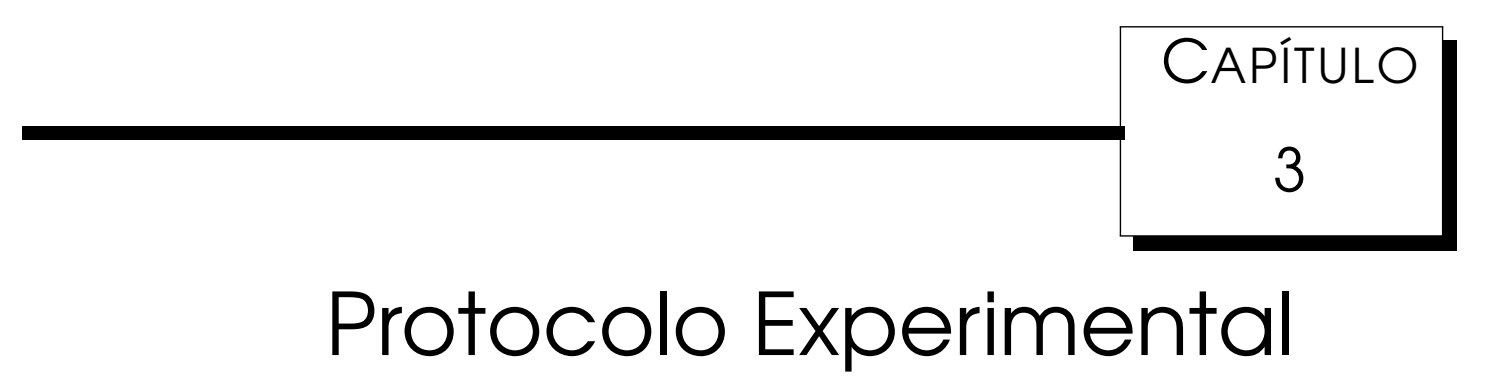

Neste capítulo, descreve-se o protocolo experimental usado na avaliação empírica realizada neste trabalho. Com o intuito de comparar os resultados obtidos neste trabalho com os da literatura (Chapelle et al., 2006), a avaliação experimental feita neste trabalho foca transdução. Nesse caso, a base de dados como um todo é usada como conjunto de treinamento usando diferentes conjuntos de exemplos rotulados e não rotulados em cada execução. Para uma avaliação empírica justa, usam-se os códigos dos autores dos algoritmos descritos neste trabalho quando possível. Como alguns dos métodos estão implementados em Matlab, usa-se a biblioteca matlabcontrol ${ }^{1}$ para que se possa usar tais códigos dentro do código fonte deste trabalho, escrito em Java. Por razões de reprodução dos resultados e para auxiliar a produção de novos trabalhos científicos na área de aprendizado semissupervisionado baseado em grafos, todos os códigos fonte e as bases de dados usados nos experimentos deste trabalho estão publicamente disponiveis ${ }^{2}$.

Este capítulo está organizado da seguinte forma. Na Seção 3.1, descrevemse as bases de dados usadas e a etapa de pré-processamento feita nas mesmas. Na Seção 3.2, descreve-se a configuração empírica usada na avaliação experimental. Na Seção 3.3, descreve-se o processo de configuração de parâmetros usado para comparar os classificadores.

\subsection{Bases de dados e pré-processamento}

As bases de dados usadas nos experimentos deste trabalho são listadas na Tabela 3.1. Dentre as bases de dados geradas por Chapelle et al. (2006), seis foram escolhidas. Elas foram escolhidas por serem publicamente disponiveis ${ }^{3}$ e amplamente usadas na literatura (Chapelle et al., 2006).

\footnotetext{
${ }^{1}$ https://code.google.com/p/matlabcontrol/downloads/list.

${ }^{2}$ http: //www.icmc.usp.br/ gbatista/ECML2013.

${ }^{3}$ http://olivier. chapelle.cc/ssl-book/benchmarks.html.
} 
Tabela 3.1: Descrição das bases de dados usadas na avaliação experimental deste trabalho. Os símbolos (a) e (r) indicam quais bases de dados são reais (r) e quais são artificiais (a).

\begin{tabular}{cccc}
\hline Data sets & \# Exemplos $(n)$ & \# Atributos $(d)$ & \# Classes $(c)$ \\
\hline USPS (r) $_{\text {COIL }}$ (r) & 1500 & 241 & 2 \\
DIGIT-1 (a) $_{\text {G-241N (a) }}^{1500}$ & 1500 & 241 & 2 \\
G-241C (a) & 1500 & 241 & 2 \\
TEXT (r) & 1500 & 241 & 2 \\
\hline
\end{tabular}

Faz-se neste trabalho uma avaliação experimental em classificação semissupervisionada em diversos domínios. USPS e DIGIT-1 são bases de dados para reconhecimento de dígitos. TEXT é uma base de dados para classificação de textos. G-241N e G-241C são bases de dados para classificação de misturas gaussianas. $\mathrm{COIL}_{2}$ é uma base de dados para classificação de imagens.

Como mostrado na Tabela 3.1, as bases de dados possuem alta dimensionalidade. Nesse caso, a distância entre cada exemplo e seu vizinho mais próximo tende à distância entre esse exemplo e seu vizinho mais distante (Beyer et al., 1999). Isso pode degenerar a qualidade do grafo a ser gerado e reduzir o desempenho de classificação dos classificadores usados. Para reduzir a dimensionalidade dos dados, usou-se o método de análise de componentes principais $\left(\mathrm{PCA}^{4}\right)$. Após experimentos preliminares, decidiu-se gerar bases de dados com 50 atributos. Para tanto, escolheu-se a implementação da biblioteca de redução de dimensionalidade do $\mathrm{Matlab}^{5}$. O método PCA não foi usado na base TEXT para manter a propriedade de esparsidade da mesma.

Escolheu-se usar as partições sugeridas por Chapelle et al. (2006). Nesse caso, cada base de dados está associada a 12 partições de exemplos rotulados e não rotulados. Com isso, o protocolo experimental fica determinístico. Logo, a reprodução dos resultados obtidos torna-se uma tarefa elementar.

\subsection{Configuração experimental}

Nesta seção, descreve-se a configuração experimental usada neste trabalho. Faz-se uma descrição dos problemas técnicos ocorridos durante experimentos preliminares e as soluções propostas para os mesmos. A configuração experimental usada neste trabalho é descrita a seguir:

Funções de distância Para a base de dados TEXT, usou-se a função de distância cosseno. Matematicamente, computa-se:

$$
\Psi\left(\boldsymbol{x}_{i}, \boldsymbol{x}_{j}\right)=1-\frac{\left\langle\boldsymbol{x}_{i}, \boldsymbol{x}_{j}\right\rangle_{d}}{\left\|\boldsymbol{x}_{i}\right\|_{2}\left\|\boldsymbol{x}_{j}\right\|_{2}}
$$

\footnotetext{
${ }^{4}$ Principal component analysis.

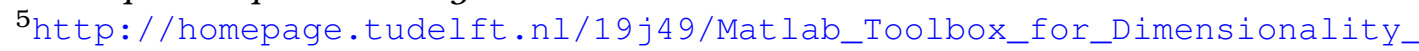
Reduction.html.
} 
O símbolo $\langle\cdot, \cdot\rangle_{d}$ representa o produto interno de vetores no espaço $\mathbb{R}^{d}$. Para as demais bases de dados, usou-se a norma $l_{2}$.

Laplacianos do grafo O Laplaciano normalizado pode levar a melhores resultados empíricos quando comparado com o Laplaciano combinatório (Johnson e Zhang, 2007). Logo, o Laplaciano normalizado foi usado na formulação dos classificadores descritos neste trabalho. Resultados insatisfatórios foram obtidos usando o Laplaciano normalizado no algoritmo RMGT em experimentos preliminares. Logo, os resultados para esse algoritmo são reportados usando o Laplaciano combinatório.

Durante experimentos preliminares, alguns erros ocorreram ao se usar o algoritmo RMGT na base de dados $\mathrm{COIL}_{2}$. Tais erros ocorreram devido ao fato de ao menos um dos autovalores do Laplaciano combinatório ser igual (ou aproximadamente) zero. Para evitar instabilidades numéricas ao resolver sistemas lineares usando os Laplacianos do grafo, gerou-se o Laplaciano combinatório por:

$$
\boldsymbol{\Delta}=\gamma \boldsymbol{D}-\boldsymbol{W}
$$

e o Laplaciano normalizado por:

$$
\boldsymbol{L}=\gamma \boldsymbol{I}_{n}-\boldsymbol{D}^{-1 / 2} \boldsymbol{W} \boldsymbol{D}^{-1 / 2}
$$

Um pequeno valor $\gamma>1$ é usado para aumentar os autovalores dos Laplacianos do grafo. Usou-se $\gamma=1.01$ nos experimentos deste trabalho.

Grafo mutKNN O procedimento $\boldsymbol{A}=\min \left(\widehat{\boldsymbol{A}}, \widehat{\boldsymbol{A}}^{\top}\right)$ pode gerar um grafo com vértices isolados. Ainda mais, o fato de haver vértices isolados no grafo pode degenerar a solução dos classificadores semissupervisionados pois o processo de difusão de rótulos pode não ser efetivo. Numa tentativa de evitar esse problema, criou-se uma aresta não orientada entre cada vértice isolado e seu vizinho mais próximo. Outras abordagens também poderiam ser usadas (Ozaki et al., 2011).

LLE Para resolver o problema de otimização em (2.8), usou-se neste trabalho o método Local Anchor Embedding (LAE) (Liu et al., 2010) ${ }^{6}$. O método LLE é um caso particular do método LAE ao se gerar um grafo bipartido cujos pontos "âncora" sejam exatamente os exemplos de treinamento. Como o algoritmo LLE pode gerar uma matriz de pesos assimétrica, simetriza-se a matriz de saída do algoritmo LLE, $\boldsymbol{W}_{L L E}$, por:

$$
\boldsymbol{W}=\frac{1}{2}\left(\boldsymbol{W}_{L L E}+\boldsymbol{W}_{L L E}^{\top}\right)
$$

\footnotetext{
${ }^{6}$ http://www.ee.columbia.edu/ln/dvmm/downloads/WeiGraphConstructCode/ dlform.htm.
} 
SymFKNN + LLE Como a matriz de adjacência do grafo symFKNN não é binária, computa-se $\widehat{\boldsymbol{W}}=\boldsymbol{W}_{L L E} \odot \boldsymbol{A}$ em que $\odot$ é o produto de Hadamard. Com isso, gera-se a matriz de pesos simétrica

$$
\boldsymbol{W}=\frac{1}{2}\left(\widehat{\boldsymbol{W}}+\widehat{\boldsymbol{W}}^{\top}\right)
$$

LapSVM O algoritmo LapSVM foi executado usando o código fonte de Melacci e Belkin $(2011)^{7}$. O algoritmo LapSVM fora treinado usando tanto o método de Newton quanto o método de gradiente conjugado pré-condicionado em experimentos preliminares. Como o método de Newton foi o método que obteve melhores resultados em experimentos preliminares, ele fora o método numérico escolhido para treinar o algoritmo LapSVM nos experimentos deste trabalho. A implementação de Melacci e Belkin (2011) resolve o problema de otimização em (2.14) usando a função de perda hinge ao quadrado, tendo em vista que a função de perda hinge não é diferenciável. Logo, os métodos de Newton e do gradiente conjugado pré-condicionado não poderiam ser usados para resolver o problema de otimização em (2.14).

LapRLS Usou-se a versão multiclasse do algoritmo LapRLS, ou seja, computou-se $\boldsymbol{\alpha}$ como a seguir:

$$
\boldsymbol{\alpha}=\left(\boldsymbol{J K}+\gamma_{A} l \mathbf{I}_{n}+\gamma_{I} l \Delta \mathbf{K}\right)^{-1} \boldsymbol{Y}
$$

Então, gerou-se a matriz de saída $\boldsymbol{F}=\boldsymbol{K} \boldsymbol{\alpha}$.

Classificação Para classificar os exemplos não rotulados, usou-se o procedimento de normalização por massas de classes $\left(\mathrm{CMN}^{8}\right)$ (Zhu et al., 2003), o qual é útil ao lidarmos com bases de dados cujos rótulos estejam desbalanceados. Dada uma matriz de saída $\boldsymbol{F} \in \mathbb{R}^{n \times c}$, o procedimento CMN classifica o exemplo $\boldsymbol{x}_{i}$ por:

$$
y\left(\boldsymbol{x}_{i}\right)=\underset{j \in \mathbb{N}_{c}}{\arg \max } \frac{\boldsymbol{\omega}_{j} \boldsymbol{F}_{i j}}{\mathbf{1}_{n}^{\top} \boldsymbol{F}_{\cdot j}}
$$

Resultados insatisfatórios foram obtidos usando CMN no algoritmo RMGT durante experimentos preliminares. Logo, os resultados para o algoritmo RMGT são reportados sem o uso do procedimento CMN. Os resultados para os classificadores GRF, LGC e LapRLS são reportados usando o procedimento CMN, enquanto que os resultados para o algoritmo LapSVM são reportados usando a função sinal. Matematicamente:

$$
\operatorname{sign}(x)=\left\{\begin{aligned}
1, & x \geq 0 \\
-1, & x<0
\end{aligned}\right.
$$

\footnotetext{
${ }^{7}$ http://www.dii.unisi.it/ melacci/lapsvmp/index.html.

${ }^{8}$ Class mass normalization.
} 
Os resultados para o algoritmo RMGT são reportados usando a função arg max. Para o algoritmo GRF, usou-se o procedimento CMN tendo como entrada a matriz $\boldsymbol{F}_{\mathcal{U}}$ ao invés da matriz $\boldsymbol{F}$, conforme sugerido por Zhu et al. (2003).

\subsection{Configuração de parâmetros}

Nesta seção, descreve-se a configuração de parâmetros dos algoritmos a serem analisados empiricamente neste trabalho. Com o intuito de evitar a explosão combinatória de experimentos e resultados, alguns parâmetros foram estimados a partir da amostra de treinamento. A configuração de parâmetros descrita neste trabalho foca a configuração dos parâmetros de esparsificação e de regularização para cada combinação de classificador e método de geração de grafos. Tal configuração é descrita a seguir:

SymKNN, mutKNN e symFKNN O parâmetro de esparsificação $k$ foi escolhido no conjunto $\{4,6,8, \cdots, 40\}$. Inicialmente, o algoritmo $b$-matching (Huang e Jebara, 2007, 2011) também seria usado nessa avaliação experimental. Como esse algoritmo atua em grafos bipartidos, os quais não possuem ciclos ímpares, valores ímpares de $k$ não poderiam ser escolhidos. Valores menores que 4 geravam grafos muito esparsos e com várias regiões isoladas enquanto que valores acima de 40 geravam grafos muito densos, o que atrapalhava o processo de difusão de rótulos. Ainda mais, a escolha de grafos muito densos pode aumentar demasiadamente o tempo de processamento.

Kernel RBF Como escolher um valor adequado para o parâmetro $\sigma$ do kernel $\mathrm{RBF}$ pode ser uma tarefa árdua quando há poucos exemplos rotulados, usou-se neste trabalho a estimativa de Jebara et al. (2009), ou seja, computou-se $\sigma$ como a seguir:

$$
\sigma=\frac{1}{3 n} \sum_{i=1}^{n} \Psi\left(\boldsymbol{x}_{i}, \boldsymbol{x}_{i_{k}}\right)
$$

Outras abordagens de estimativa do valor de $\sigma$ também poderiam ser usadas (Zelnik-Manor e Perona, 2004; Wang et al., 2008).

Matriz de kernel Criou-se a matriz de kernel $\boldsymbol{K}$ usando o kernel RBF, ou seja, computou-se $\mathbf{K}$ como a seguir:

$$
\mathbf{K}_{i j}=\exp \left(-\frac{\Psi^{2}\left(\boldsymbol{x}_{i}, \boldsymbol{x}_{j}\right)}{2 \sigma^{2}}\right)
$$

$\forall i, j \in \mathbb{N}_{n}$. Usou-se a mesma função de distância $\Psi(\cdot, \cdot)$ e o mesmo parâmetro de esparsificação $k$ usado durante a geração do grafo ponderado para estimar o valor de $\sigma$. 
LGC O parâmetro de regularização $\mu$ do algoritmo LGC foi escolhido de tal forma que $\mu \in\{0.01,0.05,0.1,0.5,1,2,5,10,50,100\}$. Valores pequenos de $\mu$ implicam uma menor confiabilidade na rotulação inicial, enquanto que valores grandes de $\mu$ implicam uma crença elevada no conhecimento do especialista. Com isso, deve-se variar o valor de $\mu$ desde valores muito pequenos até valores razoavelmente grandes. Em experimentos preliminares, a rotulação inicial passou a ter uma influência muito reduzida no processo de classificação para valores abaixo de 0.01 , enquanto que, para valores maiores que 100 , os rótulos inciais tendiam a se manter, independentemente dos dados iniciais.

LapRLS e LapSVM Os parâmetros de regularização $\gamma_{A}$ e $\gamma_{I}$ foram escolhidos de tal forma que $\gamma_{A}, \gamma_{I} \in\left\{10^{-6}, 10^{-4}, 10^{-2}, 10^{-1}, 1,10,100\right\}$, conforme sugerido por Melacci e Belkin (2011). Os demais parâmetros necessários para executar o código de Melacci e Belkin (2011), tais como o número máximo de iterações e a decomposição matricial a ser usada, foram fixados nas suas configurações padrão.

RMGT Para o algoritmo RMGT, assume-se uma distribuição de classes uniforme. Configura-se $\boldsymbol{\omega}=\mathbf{1}_{c} / c$ ao invés de usar as probabilidades a priori das classes, conforme sugerido por Liu e Chang (2009). Em experimentos preliminares, os resultados usando a distribuição de classes uniforme obtiveram as menores taxas de erro em relação aos resultados usando-se as probabilidades a priori das classes. Logo, reportam-se os resultados para o algoritmo RMGT usando essa configuração. Para a base USPS, reportam-se os resultados para o algoritmo RMGT usando as probabilidades a priori das classes.

\subsection{Considerações Finais}

Este capítulo descreveu o protocolo experimental usado neste trabalho. Os resultados mostrados nos Capítulos 4, 5, 6 e 7 seguem o protocolo experimental descrito neste capítulo. Acredita-se que uma descrição detalhada do protocolo experimental seja de suma importância para viabilizar o processo de replicação dos resultados. Ainda mais, o rigor experimental faz-se necessário para evitar a geração de conclusões tendenciosas a partir dos resultados obtidos. 


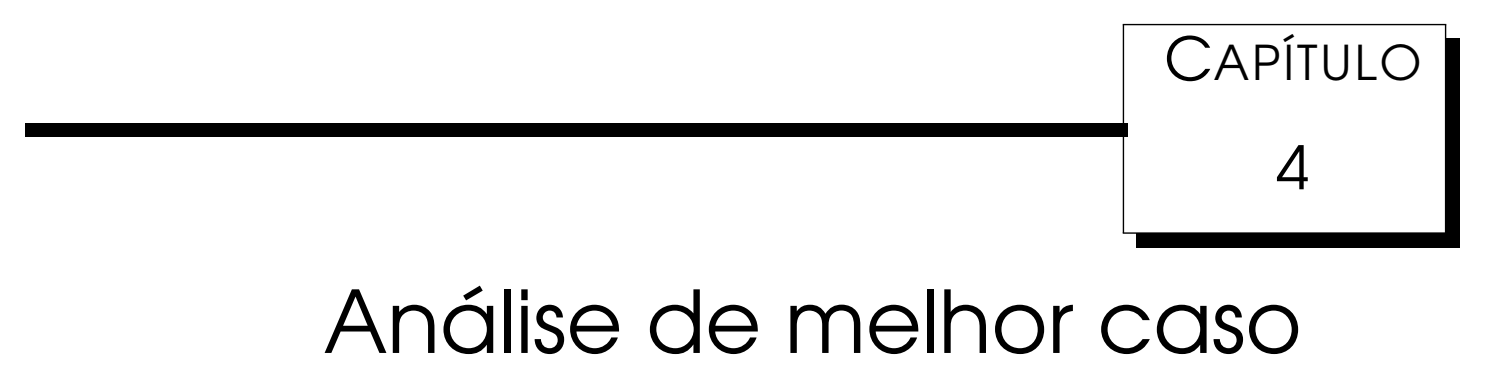

Neste capítulo, faz-se uma avaliação experimental dos classificadores semissupervisionados combinados com os métodos de geração de grafos com base nas melhores taxas de erro obtidas para cada classificador. A avaliação é feita com base nas melhores taxas de erro de cada combinação de classificador semissupervisionado com cada método de geração de grafos usando o protocolo experimental descrito no capítulo anterior. Essa forma de avaliação é amplamente usada na literatura de aprendizado semissupervisionado (Zhu, 2005; Chapelle et al., 2006; Melacci e Belkin, 2011; Kong et al., 2012; Wang e Xia, 2012; Luo et al., 2012; Ghazvininejad et al., 2011; Zhuang et al., 2012; Nie et al., 2011; Urner et al., 2011; Zhang et al., 2011). Entretanto, é mostrado nos capítulos posteriores que tal análise pode não prover toda a informação necessária para escolher os melhores classificadores para uma dada aplicação real, tendo em vista que classificadores que apresentam boa estabilidade podem ser preferiveis em relação à classificadores que apresentam excelentes desempenhos de classificação em apenas determinados casos.

Este capítulo está organizado como a seguir. Na Seção 4.1, avaliam-se os resultados obtidos para a análise de melhor caso. Na Seção 4.2, discutem-se as considerações finais deste capítulo.

\subsection{Avaliação dos resultados obtidos}

Os resultados mostrados neste capítulo foram obtidos com o uso do protocolo experimental descrito no Capítulo 3. As menores taxas de erro de cada classificador em relação a todos os valores de parâmetros são reportadas nas Tabelas 4.1 e 4.3 para as partições de 10 e de 100 exemplos rotulados, respectivamente. Os quatro piores resultados para cada classificador em cada base de dados possuem um fundo cinza enquanto que o melhor resultado está em negrito. O melhor resultado geral para cada base de dados está em um retângulo. 
Tabela 4.1: Médias das taxas de erro (\%) e desvios-padrão (\%) dos classificadores semissupervisionados baseados em grafos nas partições de 10 exemplos rotulados.

\begin{tabular}{|c|c|c|c|c|c|c|}
\hline Data sets & USPS & $\mathrm{COIL}_{2}$ & DIGIT-1 & G-241N & G-241C & TEXT \\
\hline GRF-symKNN-RBF & $11.07(3.33)$ & $35.13(6.92)$ & $10.19(4.27)$ & $46.12(7.61)$ & $46.28(6.98)$ & 39.15 (5.69) \\
\hline GRF-mutKNN-RBF & $9.75(4.50)$ & $35.07(3.82)$ & $9.35(4.51)$ & $46.94(4.81)$ & $46.72(4.85)$ & $37.51(6.85)$ \\
\hline GRF-symFKNN-RBF & $10.75(3.77)$ & $35.22(6.92)$ & $10.01(3.93)$ & $46.12(7.65)$ & $46.34(6.83)$ & $38.50(5.87)$ \\
\hline GRF-symKNN-HM & $15.53(2.76)$ & $38.55(6.06)$ & $10.73(4.27)$ & $46.86(5.28)$ & $46.19(7.25)$ & $42.32(8.54)$ \\
\hline GRF-mutKNN-HM & $11.01(3.59)$ & $35.30(3.87)$ & $10.02(6.36)$ & $46.66(6.18)$ & $46.58(5.06)$ & $41.18(9.87)$ \\
\hline GRF-symFKNN-HM & $15.17(3.09)$ & $37.77(6.25)$ & $10.24(4.36)$ & $46.77(5.38)$ & 46.27 (7.05) & $42.20(8.62)$ \\
\hline GRF-symKNN-LLE & $16.03(2.47)$ & $36.04(5.60)$ & $10.94(4.69)$ & $47.54(3.77)$ & $47.33(4.77)$ & $43.56(6.96)$ \\
\hline GRF-mutKNN-LLE & $11.64(3.39)$ & $35.20(3.79)$ & $10.30(5.89)$ & $47.14(2.87)$ & 46.98 (3.64) & $42.34(6.48)$ \\
\hline GRF-symFKNN-LLE & $15.55(2.74)$ & $36.10(5.88)$ & $10.31(4.68)$ & $47.25(4.14)$ & $47.46(4.36)$ & $43.54(6.95)$ \\
\hline LGC-symKNN-RBF & $11.22(3.07)$ & 34.96 (6.69) & $10.68(4.91)$ & $38.06(6.91)$ & $40.24(5.13)$ & $35.42(5.58)$ \\
\hline LGC-mutKNN-RBF & $9.93(4.34)$ & $35.07(3.82)$ & $10.54(5.21)$ & $39.82(5.36)$ & $41.85(4.32)$ & $34.78(6.55)$ \\
\hline LGC-symFKNN-RBF & $10.97(3.00)$ & $34.81(6.22)$ & $10.47(4.66)$ & $37.95(6.66)$ & $40.10(5.46)$ & $35.51(5.64)$ \\
\hline LGC-symKNN-HM & $14.49(5.25)$ & $37.20(7.32)$ & $11.53(5.00)$ & $38.36(6.83)$ & $40.27(4.48)$ & $37.51(4.48)$ \\
\hline LGC-mutKNN-HM & 10.79 (3.75) & $35.19(4.90)$ & $10.96(5.34)$ & $39.51(5.80)$ & $41.94(4.20)$ & $36.01(5.63)$ \\
\hline LGC-symFKNN-HM & $14.63(3.33)$ & $36.36(8.25)$ & $11.07(4.76)$ & $38.13(7.11)$ & $40.17(4.75)$ & $37.49(4.35)$ \\
\hline LGC-symKNN-LLE & $15.05(4.33)$ & $35.95(6.09)$ & $11.49(5.41)$ & $41.22(4.12)$ & $43.33(3.03)$ & $39.18(4.02)$ \\
\hline LGC-mutKNN-LLE & $11.04(3.82)$ & $35.18(3.77)$ & $10.96(6.34)$ & $42.12(3.90)$ & 42.94 (3.09) & $35.89(9.20)$ \\
\hline LGC-symFKNN-LLE & $14.51(2.81)$ & $35.98(6.07)$ & $10.97(5.01)$ & $41.24(4.37)$ & $43.06(3.34)$ & $39.03(3.82)$ \\
\hline LapRLS-symKNN-RBF & $10.99(3.05)$ & $34.92(5.98)$ & $10.22(4.25)$ & $38.09(6.76)$ & $40.35(6.23)$ & $35.12(5.68)$ \\
\hline LapRLS-mutr & $9.75(4.53)$ & $33.56(7.32)$ & $9.33(4.48)$ & $38.36(5.96)$ & $40.66(5.45)$ & $34.58(6.14)$ \\
\hline LapRLS-symFKNN-RBF & $10.57(2.90)$ & $35.50(5.84)$ & $10.02(3.92)$ & $38.08(6.64)$ & $40.36(6.02)$ & $35.34(5.73)$ \\
\hline LapRLS-symKNN-HM & $14.56(3.89)$ & $37.58(5.91)$ & $10.76(4.24)$ & $38.18(6.70)$ & $40.24(6.07)$ & $37.12(4.52)$ \\
\hline LapRLS-mutKNN-HM & $10.57(4.66)$ & $32.80(7.67)$ & $9.92(5.50)$ & $38.29(6.00)$ & $40.67(5.50)$ & $35.90(5.61)$ \\
\hline LapRLS-symFKNN-HM & $14.38(4.14)$ & $36.93(4.95)$ & $10.28(4.32)$ & $38.06(6.52)$ & $40.11(6.06)$ & $37.32(4.38)$ \\
\hline LapRLS-symKNN-LLE & $14.73(3.24)$ & $36.85(5.25)$ & $10.93(4.66)$ & $38.68(5.60)$ & $40.61(5.51)$ & $38.49(4.00)$ \\
\hline LapRLS-mutKNN-LLE & $11.28(4.09)$ & $31.78(7.81)$ & $10.19(5.92)$ & $38.66(5.72)$ & $40.59(5.61)$ & $37.28(5.26)$ \\
\hline LapRLS-symFKNN-LLE & $14.55(3.37)$ & $36.17(4.81)$ & $10.31(4.63)$ & $38.69(5.56)$ & $40.61(5.52)$ & $38.62(4.13)$ \\
\hline LapSVM-symKNN-RBF & $11.42(4.03)$ & $34.96(6.81)$ & $9.42(3.97)$ & $39.16(6.07)$ & $40.91(6.08)$ & $39.88(6.02)$ \\
\hline LapSVM-mu & $9.91(2.51)$ & $34.37(6$. & $8.67(3.89)$ & $38.90(6.50)$ & $40.90(6.08)$ & $37.49(7.07)$ \\
\hline LapSVM-symFKNN-RBF & $11.04(3.43)$ & $34.04(6.92)$ & $9.47(4.19)$ & $39.16(6.07)$ & $40.91(6.08)$ & $39.45(6.30)$ \\
\hline LapSVM-symKNN-HM & $14.63(5.47)$ & $36.40(4.07)$ & $10.13(3.65)$ & $39.15(6.04)$ & $40.91(6.08)$ & $43.06(4.99)$ \\
\hline LapSVM-mutKNN-HM & $10.04(2.83)$ & 33.08 (6.35) & $9.58(4.73)$ & $39.00(6.43)$ & $40.90(6.08)$ & $42.10(6.03)$ \\
\hline LapSVM-symFKNN-HM & $14.35(4.29)$ & $36.57(3.57)$ & $9.93(3.95)$ & $39.14(6.05)$ & $40.91(6.08)$ & $42.71(5.15)$ \\
\hline LapSVM-symKNN-LLE & $14.82(3.38)$ & $35.39(4.80)$ & $10.31(4.11)$ & $39.12(6.43)$ & $40.90(6.07)$ & $42.77(6.20)$ \\
\hline LapSVM-mutKNN-LLE & $10.61(2.49)$ & $31.54(6.24)$ & $10.22(5.52)$ & $38.95(6.46)$ & $40.82(6.66)$ & $41.80(7.65)$ \\
\hline LapSVM-symFKNN-LLE & $14.41(3.23)$ & $35.21(4.58)$ & $9.83(3.99)$ & $39.00(6.40)$ & $40.84(6.40)$ & $42.62(4.92)$ \\
\hline RMGT-symKNN-RBF & $16.62(2.90)$ & $31.05(4.81)$ & $8.63(3.35)$ & $44.99(6.97)$ & $38.44(6.22)$ & $30.43(6.26)$ \\
\hline RMGT-mutKNN-RBF & $13.08(3.41)$ & $28.95(3.88)$ & $8.13(3.14)$ & $46.11(4.50)$ & $42.76(6.11)$ & 27.77 (5.95) \\
\hline RMGT-symFKNN-RBF & $16.02(2.85)$ & $32.94(4.20)$ & $8.55(3.36)$ & $45.25(6.07)$ & $38.31(6.02)$ & $29.65(6.46)$ \\
\hline RMGT-symKNN-HM & $19.08(1.22)$ & $31.20(6.14)$ & $8.07(2.69)$ & $44.31(9.03)$ & $38.48(6.91)$ & $34.86(6.04)$ \\
\hline RMGT-mutKNN-HM & $16.99(2.45)$ & $28.00(4.67)$ & $7.50(2.43)$ & $44.73(5.48)$ & $40.53(4.37)$ & $31.12(6.35)$ \\
\hline RMGT-symFKNN-HM & $18.88(2.26)$ & $30.56(5.52)$ & $7.92(2.58)$ & $44.68(7.89)$ & $38.48(6.67)$ & $34.61(6.25)$ \\
\hline RMGT-symKNN-LLE & $19.04(1.19)$ & $30.63(3.94)$ & $7.91(2.49)$ & $42.83(6.00)$ & $42.25(3.32)$ & $36.61(4.79)$ \\
\hline RMGT-mutKNN-LLE & $17.85(1.95)$ & $29.49(4.16)$ & $7.53(2.11)$ & $43.75(6.40)$ & $42.12(4.08)$ & $33.89(5.32)$ \\
\hline RMGT-symFKNN-LLE & $18.97(1.18)$ & $30.41(3.71)$ & $7.73(2.43)$ & $42.75(7.33)$ & 41.77 (3.33) & $36.25(4.78)$ \\
\hline
\end{tabular}

Tabela 4.2: Rankings médios para os métodos de geração de grafos nas partições de 10 exemplos rotulados em relação a cada classificador semissupervisionado e a média geral dos rankings.

\begin{tabular}{|c|ccccc|c|}
\cline { 2 - 7 } \multicolumn{1}{c|}{} & GRF & LGC & LapRLS & LapSVM & RMGT & geral \\
\hline symKNN-RBF & 2.9167 & 2.8333 & 3.5 & 5.1667 & 5.1667 & 3.9167 \\
mutKNN-RBF & 2.6667 & 3 & $\mathbf{3 . 1 6 6 7}$ & $\mathbf{2}$ & 4.8333 & 3.1333 \\
symFKNN-RBF & $\mathbf{2 . 5 8 3 3}$ & $\mathbf{1 . 6 6 6 7}$ & 3.25 & 4.6667 & 5 & 3.4333 \\
symKNN-HM & 6 & 6.5 & 6 & 7.75 & 6.25 & 6.5 \\
mutKNN-HM & 3.8333 & 4.5833 & 4.25 & 3.4167 & $\mathbf{3 . 5}$ & 3.9167 \\
symFKNN-HM & 5 & 5.6667 & 4.8333 & 6.9167 & 5.0833 & 5.5 \\
symKNN-LLE & 8.3333 & 8 & 7.9167 & 7 & 6.1667 & 7.4833 \\
mutKNN-LLE & 5.8333 & 5.4167 & 4.6667 & 3.1667 & 4.1667 & 4.65 \\
symFKNN-LLE & 7.8333 & 7.3333 & 7.4167 & 4.9167 & 4.8333 & 6.4667 \\
\hline
\end{tabular}


Tabela 4.3: Médias das taxas de erro (\%) e desvios-padrão (\%) dos classificadores semissupervisionados baseados em grafos nas partições de 100 exemplos rotulados.

\begin{tabular}{|c|c|c|c|c|c|c|}
\hline Data sets & USPS & $\mathrm{COIL}_{2}$ & DIGIT-1 & G-241N & G-241C & TEXT \\
\hline GRF-symKNN-RBF & $3.30(0.97)$ & $1.14(1.46)$ & $2.22(0.52)$ & $32.38(5.69)$ & $37.89(5.60)$ & $24.79(2.89)$ \\
\hline GRF-mutKNN-RBF & $3.33(1.05)$ & 1.09 (1.59) & $2.17(0.37)$ & $32.56(5.28)$ & $38.62(5.58)$ & $23.69(2.48)$ \\
\hline GRF-symFKNN-RBF & $3.26(1.00)$ & $1.14(1.46)$ & $2.19(0.48)$ & $32.17(5.70)$ & 37.59 (5.75) & $24.12(3.13)$ \\
\hline GRF-symKNN-HM & $6.30(2.30)$ & $3.80(1.75)$ & $2.01(0.58)$ & $31.75(5.79)$ & 37.78 (5.59) & $28.48(3.08)$ \\
\hline GRF-mutKNN-HM & $3.78(1.32)$ & $2.27(1.70)$ & $2.10(0.53)$ & $32.42(5.29)$ & $38.96(6.06)$ & $25.29(2.40)$ \\
\hline GRF-symFKNN-HM & $5.92(2.19)$ & $3.02(1.64)$ & $2.04(0.52)$ & $31.71(6.08)$ & $37.68(5.60)$ & $27.62(3.87)$ \\
\hline GRF-symKNN-LLE & $7.44(2.35)$ & 2.06 (1.79) & $2.18(0.53)$ & $33.13(4.39)$ & $38.65(4.76)$ & 28.79 (3.06) \\
\hline GRF-mutKNN-LLE & $4.48(1.55)$ & $1.34(1.49)$ & $2.21(0.64)$ & $33.78(4.64)$ & $39.12(3.65)$ & $25.86(2.37)$ \\
\hline GRF-symFKNN-LLE & $6.76(2.30)$ & $1.29(1.57)$ & $2.18(0.44)$ & $32.85(4.42)$ & $38.59(4.66)$ & $27.92(3.82)$ \\
\hline LGC-symKNN-RBF & $3.57(1.00)$ & $1.17(1.49)$ & $2.46(0.65)$ & $22.38(1.58)$ & $29.98(2.91)$ & $24.26(2.18)$ \\
\hline LGC-mutKNN-RBF & $3.58(0.99)$ & 1.09 (1.59) & $2.43(0.43)$ & $26.20(2.58)$ & $34.45(2.89)$ & $23.35(2.26)$ \\
\hline LGC-symFKNN-RBF & $3.54(0.96)$ & $1.14(1.48)$ & $2.45(0.61)$ & $22.82(2.48)$ & $30.42(2.46)$ & $23.59(1.42)$ \\
\hline LGC-symKNN-HM & $5.66(0.96)$ & $4.10(1.97)$ & $2.31(0.52)$ & $22.10(1.65)$ & $29.81(2.57)$ & $27.71(2.84)$ \\
\hline LGC-mutKNN-HM & $3.97(0.87)$ & $2.22(1.52)$ & $2.35(0.62)$ & $26.17(3.01)$ & $34.70(2.74)$ & $25.01(2.60)$ \\
\hline LGC-symFKNN-HM & $5.40(0.74)$ & $3.52(2.08)$ & $2.13(0.72)$ & 22.04 (1.75) & $30.21(3.27)$ & $26.78(1.37)$ \\
\hline LGC-symKNI & $6.45(0.77)$ & $2.36(1.97)$ & $2.37(0.91)$ & $29.07(1.75)$ & $34.36(2.56)$ & $28.24(2.97)$ \\
\hline LGC-mutKNN-LLE & $4.34(0.81)$ & $1.43(1.55)$ & $2.32(0.72)$ & $30.22(2.11)$ & $36.13(2.40)$ & 24.25 (6.24) \\
\hline LGC-symFKNN-LLE & $5.91(0.79)$ & $1.62(1.81)$ & $2.38(0.85)$ & $28.93(1.80)$ & $34.20(2.63)$ & $27.13(2.68)$ \\
\hline LapRLS-syr & $3.29(1.01)$ & $0.80(1.13)$ & $2.17(0.43)$ & $22.14(1.59)$ & $28.73(3.43)$ & $24.36(1.58)$ \\
\hline LapRLS-mutKNN-RBF & $3.28(0.98)$ & $0.39(0.92)$ & $2.05(0.41)$ & $23.92(2.15)$ & $28.73(3.43)$ & $23.48(1.64)$ \\
\hline LapRLS-symFKNN-RBF & $3.17(0.93)$ & $0.78(1.13)$ & $2.14(0.37)$ & $22.74(1.79)$ & $28.73(3.43)$ & $23.65(1.66)$ \\
\hline LapRLS-symKNN-HM & $5.32(1.10)$ & $3.34(1.52)$ & $1.87(0.51)$ & $21.89(1.73)$ & $28.72(3.43)$ & $27.20(2.97)$ \\
\hline LapRLS-mutKNN-HM & $3.73(1.25)$ & $0.61(0.88)$ & $2.03(0.38)$ & $23.95(2.13)$ & $28.73(3.43)$ & $24.84(1.90)$ \\
\hline LapRLS-sy & $4.87(1.04)$ & $2.62(1$ & $1.95(0.55)$ & $21.99(1.75)$ & $28.72(3.43)$ & $26.82(1.83)$ \\
\hline LapRLS-symKNN-LLE & $5.98(1.22)$ & $1.69(1.30)$ & $2.12(0.57)$ & $23.96(2.17)$ & $28.73(3.44)$ & $27.20(2.93)$ \\
\hline LapRLS-m & $4.14(0.88)$ & $0.35(0.75)$ & $2.14(0.44)$ & $23.96(2.18)$ & $28.73(3.43)$ & $25.33(1.66)$ \\
\hline LapRLS-symFKNN-LLE & $5.48(1.01)$ & $0.89(0.71)$ & $2.12(0.47)$ & $23.96(2.17)$ & $28.73(3.44)$ & $26.93(2.60)$ \\
\hline LapSVM-symKNN-RBF & $4.10(1.46)$ & $0.80(1.14)$ & $2.18(0.47)$ & $21.64(3.90)$ & $25.51(5.58)$ & $23.74(2.10)$ \\
\hline & $71(1.44)$ & & 2.13( & 21.64 & 25.51 & $22.43(1.11)$ \\
\hline LapSVM-sy & $3.94(1.73)$ & $0.79(1.14)$ & $2.14(0.39)$ & $21.64(3.90)$ & $25.51(5.58)$ & $23.09(1.81)$ \\
\hline LapSVM-symKNN-HM & $5.74(1.38)$ & $3.35(1.58)$ & $1.92(0.48)$ & $21.64(3.90)$ & $25.51(5.58)$ & $27.67(2.57)$ \\
\hline LapSVM-mutKNN-HM & $3.71(1.26)$ & $0.65(0.90)$ & $2.02(0.40)$ & $21.64(3.90)$ & $25.51(5.58)$ & $24.66(1.62)$ \\
\hline LapSVM-symFKNN-HM & $5.30(1.19)$ & $2.72(1.56)$ & $1.93(0.54)$ & $21.64(3.90)$ & $25.51(5.58)$ & $26.50(2.13)$ \\
\hline LapSVM-s & $6.37(0.97)$ & & & $21.64(3.90)$ & $25.52(5.58)$ & $28.55(2.45)$ \\
\hline LapSVM-m & $4.18(1.19)$ & $0.36(0.74)$ & $2.11(0.41)$ & $21.64(3.90)$ & $25.51(5.58)$ & $25.57(2.40)$ \\
\hline LapSVM-symFKNN-LLE & $5.90(1.41)$ & $0.90(0.95)$ & $2.13(0.60)$ & $21.64(3.80)$ & $25.51(5.58)$ & $27.89(3.66)$ \\
\hline RMGT-syn & $6.57(2.24)$ & $1.92(1.74)$ & $2.38(0.43)$ & $28.21(8.55)$ & $25.87(2.39)$ & $21.00(1.25)$ \\
\hline RMGT-mu & $4.85(1.83)$ & $1.95(1.59)$ & $2.32(0.37)$ & $31.08(5.09)$ & $33.12(3.51)$ & $20.89(1.07)$ \\
\hline RMGT-symFKNN-RBF & $6.15(2.10)$ & $1.72(1.63)$ & $2.35(0.40)$ & $28.40(8.28)$ & $27.02(2.25)$ & $20.76(1.18)$ \\
\hline RMGT-symKNN-HM & $8.32(2.37)$ & $2.93(1.19)$ & $1.95(0.40)$ & $26.27(8.72)$ & $25.11(2.12)$ & $24.73(1.36)$ \\
\hline RMGT-mutKNN-HM & $4.71(2.25)$ & $2.41(1.49)$ & $2.07(0.49)$ & $29.64(6.28)$ & $32.02(2.78)$ & $22.48(1.08)$ \\
\hline RMGT-symFKNN-HM & $7.71(2.52)$ & $2.11(1.05)$ & $1.97(0.53)$ & $26.01(8.82)$ & $25.49(2.21)$ & $23.47(1.06)$ \\
\hline & $9.59(2.52)$ & $1.42(1.02)$ & $2.07(0.39)$ & $28.64(3.15)$ & $31.64(1.70)$ & $24.29(1.53)$ \\
\hline RMGT-mutKNN-LLE & $5.84(2.39)$ & $0.97(0.76)$ & $2.11(0.29)$ & $29.93(4.80)$ & $34.10(2.10)$ & $22.73(1.09)$ \\
\hline RMGT-symFKNN-LLE & $8.96(2.63)$ & $0.85(0.80)$ & $1.99(0.43)$ & $28.47(4.00)$ & $31.92(1.50)$ & $23.17(1.45)$ \\
\hline
\end{tabular}

Tabela 4.4: Rankings médios para os métodos de geração de grafos nas partições de 100 exemplos rotulados em relação a cada classificador semissupervisionado e a média geral dos rankings.

\begin{tabular}{|c|ccccc|c|}
\cline { 2 - 7 } \multicolumn{1}{c|}{} & GRF & LGC & LapRLS & LapSVM & RMGT & geral \\
\hline symKNN-RBF & 4.0833 & 3.8333 & 4.8333 & 5.0833 & 4.3333 & 4.4333 \\
mutKNN-RBF & 3.5 & 4.1667 & $\mathbf{3 . 3 3 3 3}$ & $\mathbf{3 . 4 1 6 7}$ & 5.5 & 3.9833 \\
symFKNN-RBF & $\mathbf{2 . 7 5}$ & $\mathbf{3 . 5}$ & 4.0833 & 4.4167 & $\mathbf{3 . 8 3 3 3}$ & 3.7167 \\
symKNN-HM & 5 & 4.8333 & 4.6667 & 5.5833 & 4.6667 & 4.95 \\
mutKNN-HM & 5.1667 & 5.3333 & 4.3333 & 3.5 & 5.75 & 4.8167 \\
symFKNN-HM & 4.1667 & 4.1667 & 4.25 & 5.25 & 4 & 4.3667 \\
symKNN-LLE & 7.4167 & 7.3333 & 7.3333 & 7.1667 & 5.5833 & 6.9667 \\
mutKNN-LLE & 6.8333 & 5.5 & 5.4167 & 4.25 & 6.3333 & 5.6667 \\
symFKNN-LLE & 6.0833 & 6.3333 & 6.75 & 6.3333 & 5 & 6.1 \\
\hline
\end{tabular}


Percebe-se na Tabela 4.1 que os grafos symKNN-LLE e symFKNN-LLE podem não ser adequados para os algoritmos GRF, LGC e LapRLS pois os resultados obtidos com essas configurações em todas as bases de dados não foram satisfatórios. Em adição, nota-se que os grafos symKNN-HM e symFKNN-HM podem não ser adequados para o classificador LapSVM pois os resultados obtidos com essas configurações de grafo não foram satisfatórios em todas as bases de dados. Para o algoritmo LapRLS, o grafo symFKNN-HM pode não ser adequado pois os resultados para essa configuração foram insatisfatórios em quatro das seis bases de dados usadas. Entretanto, essa configuração de grafo obteve o melhor resultado para o algoritmo LapRLS nas bases G-241C e G-241N.

Ainda na Tabela 4.1, pode-se perceber que o grafo mutKNN supera os grafos symKNN e symFKNN na maioria das situações, independentemente do método de geração de matrizes ponderadas e do classificador semissupervisionado usados. Logo, para as bases de dados consideradas neste trabalho, o grafo mutKNN apresentou o melhor desempenho de classificação dentre os métodos de geração de grafos de adjacência.

Para comparar estatisticamente os resultados obtidos para os métodos de geração de grafos, executou-se o teste de Friedman ${ }^{1}$ com o pós-teste de Nemenyi usando um nível de significância 0.05. Nas Tabelas 4.2 e 4.4, mostramse os rankings médios dos métodos de geração de grafos em relação a cada classificador semissupervisionado. Os melhores resultados de rankings estão em negrito e os resultados que são estatisticamente inferiores ao método que obteve o melhor ranking são marcados com fundo cinza. A partir das Tabelas 4.2 e 4.4, nota-se que os grafos mutKNN-RBF e symFKNN-RBF obtiveram os melhores rankings médios para a maioria dos classificadores semissupervisionados. Entretanto, o teste estatístico usado só conseguiu evidenciar diferenças estatisticamente significativas em 7 casos para as partições de 10 exemplos rotulados, como mostrado na Tabela 4.2. Para as partições de 100 exemplos rotulados, o teste estatístico não encontrou nenhuma diferença estatisticamente significativa, conforme mostrado na Tabela 4.4.

Na Tabela 4.1, nota-se que o algoritmo RMGT obteve o melhor desempenho de classificação geral em 4 das 6 bases de dados. Na Tabela 4.3, percebe-se que o algoritmo RMGT obteve o melhor desempenho de classificação em apenas duas bases de dados. Entretanto, nota-se que os resultados do algoritmo RMGT para as bases USPS, COIL 2 e DIGIT-1 são bastante competitivos com os melhores resultados gerais para essas bases de dados.

$\mathrm{Na}$ Tabela 4.3, nota-se que os resultados obtidos para as bases $\mathrm{COIL}_{2} \mathrm{e}$ DIGIT-1 são bastante próximos do melhor resultado geral para essas bases de dados na maioria dos casos. Com isso, poder-se-ia escolher qualquer classificador semissupervisionado combinado com qualquer método de geração de grafos para resolver esses problemas de reconhecimento de dígitos e de classificação de imagens.

Nota-se na Tabela 4.3 que o algoritmo LapRLS apresentou desempenho

\footnotetext{
${ }^{1}$ Veja o trabalho de Demšar (2006) para uma revisão em testes estatísticos para o aprendizado de máquina.
} 
de classificação equivalente para todos os métodos de geração de grafos para as bases G-241N e G-241C. Apesar de os resultados para cada conjunto de parâmetros serem diferentes entre si, os resultados para a análise de melhor caso são equivalentes. Entretanto, esses resultados foram obtidos usando diferentes valores para os parâmetros de esparsificação e de regularização.

Com base nas Tabelas 4.1 e 4.3, percebe-se que o classificador RMGT não obteve um bom desempenho de classificação na base de dados USPS usando os métodos de geração de matrizes ponderadas HM e LLE. Ainda mais, o classificador RMGT não é competitivo com os demais classificadores na base USPS para nenhuma das configurações de grafo usadas nos experimentos deste trabalho para as partições de 10 exemplos rotulados, conforme mostrado na Tabela 4.1. Entretanto, para as partições de 100 exemplos rotulados, o desempenho de classificação do algoritmo RMGT passa a ser competitivo com os demais classificadores ao se usar o grafo mutKNN, conforme mostrado na Tabela 4.3.

Com base na Tabela 4.2, nota-se que grafos construídos com o kernel RBF obtiveram o melhor ranking médio no geral. Com isso, o kernel RBF parece ser a melhor opção para se gerar matrizes ponderadas nesse caso. Ainda mais, nota-se que o grafo mutKNN obteve os melhores rankings médios para todos os métodos de geração de matrizes ponderadas. Em adição, nota-se na Tabela 4.2 que os rankings referentes ao grafo mutKNN são melhores que os rankings referentes aos grafos symKNN e symFKNN para os métodos HM e LLE para todos os classificadores semissupervisionados. Entretanto, esse fato não é evidenciado ao se usar as partições de 100 exemplos rotulados, conforme mostrado na Tabela 4.4.

Na Tabela 4.2, percebe-se que os resultados para o kernel RBF são superiores aos resultados para os métodos HM e LLE em relação aos classificadores GRF, LGC, LapRLS e LapSVM. Entretanto, essa superioridade não é evidenciada para o classificador RMGT. Como mostrado na Tabela 4.1, os resultados para o classificador RMGT usando o kernel RBF foram piores que as outras configurações de grafos em vários casos.

Na Tabela 4.5, comparam-se os piores e os melhores resultados obtidos nos experimentos deste trabalho com os resultados da literatura (Chapelle et al., 2006). Chapelle et al. (2006) usaram uma variedade de classificadores supervisionados e semissupervisionados com uma variedade de valores para os parâmetros dos classificadores para avaliar o desempenho de classificação dos mesmos nas bases de dados usadas neste trabalho. Alguns desses classificadores foram projetados para domínios específicos, tais como reconhecimento de dígitos e classificação de misturas gaussianas. Com base na Tabela 4.5, percebe-se que os resultados obtidos neste trabalho superam alguns dos resultados obtidos na literatura. Entretanto, para as bases de dados artificiais G-241N e G-241C, os resultados da literatura são bastante superiores aos obtidos neste trabalho. Isso se deve ao fato de Chapelle et al. (2006) terem usado métodos específicos para classificação de misturas gaussianas no processo de avaliação experimental. 
Tabela 4.5: Os piores $(p)$ e os melhores $(m)$ resultados para as partições de 10 e de 100 exemplos rotulados apresentados por Chapelle et al. (2006) para as bases de dados usadas nos experimentos deste trabalho. Os piores e melhores resultados obtidos neste trabalho são reportados por $p^{*}$ e $m^{*}$, respectivamente.

\begin{tabular}{|c|c|c|c|c|c|c|c|c|}
\cline { 2 - 8 } \multicolumn{1}{c|}{} & \multicolumn{9}{c|}{10} & \multicolumn{4}{c|}{100} \\
\cline { 2 - 9 } \multicolumn{1}{c|}{} & $p$ & $p^{*}$ & $m$ & $m^{*}$ & $p$ & $p^{*}$ & $m$ & $m^{*}$ \\
\hline USPS & 25.36 & 19.08 & 13.61 & 9.75 & 9.77 & 9.59 & 4.68 & 3.17 \\
COIL 2 & - & 38.55 & - & 31.54 & - & 4.10 & - & 0.35 \\
DIGIT-1 & 30.60 & 11.53 & 5.44 & 7.50 & 6.15 & 2.46 & 2.44 & 1.87 \\
G-241N & 50.63 & 47.54 & 18.64 & 37.95 & 43.21 & 33.78 & 4.95 & 21.64 \\
G-241C & 49.59 & 47.46 & 22.76 & 38.31 & 44.05 & 39.12 & 13.49 & 25.11 \\
TEXT & 45.37 & 43.56 & 27.15 & 27.77 & 30.92 & 28.79 & 23.09 & 20.76 \\
\hline
\end{tabular}

\subsection{Considerações finais}

Este capítulo avaliou os resultados obtidos para a análise de melhor caso. Devido à alta variabilidade dos resultados, não foi possível identificar um padrão de comportamento que pudesse auxiliar o processo de escolha de parâmetros. A partir dos resultados obtidos, pode-se concluir que o grafo mutKNN pode ser a melhor opção dentre os métodos de geração de grafos de adjacência, enquanto que o kernel RBF pode ser a melhor opção dentre os métodos de geração de matrizes ponderadas. Como mencionado no Capítulo 1, a análise de melhor caso por si pode não ser suficiente para auxiliar a escolha dos melhores classificadores para aplicações reais. No próximo capítulo, faz-se uma análise dos resultados obtidos para a avaliação da estabilidade dos classificadores semissupervisionados. 


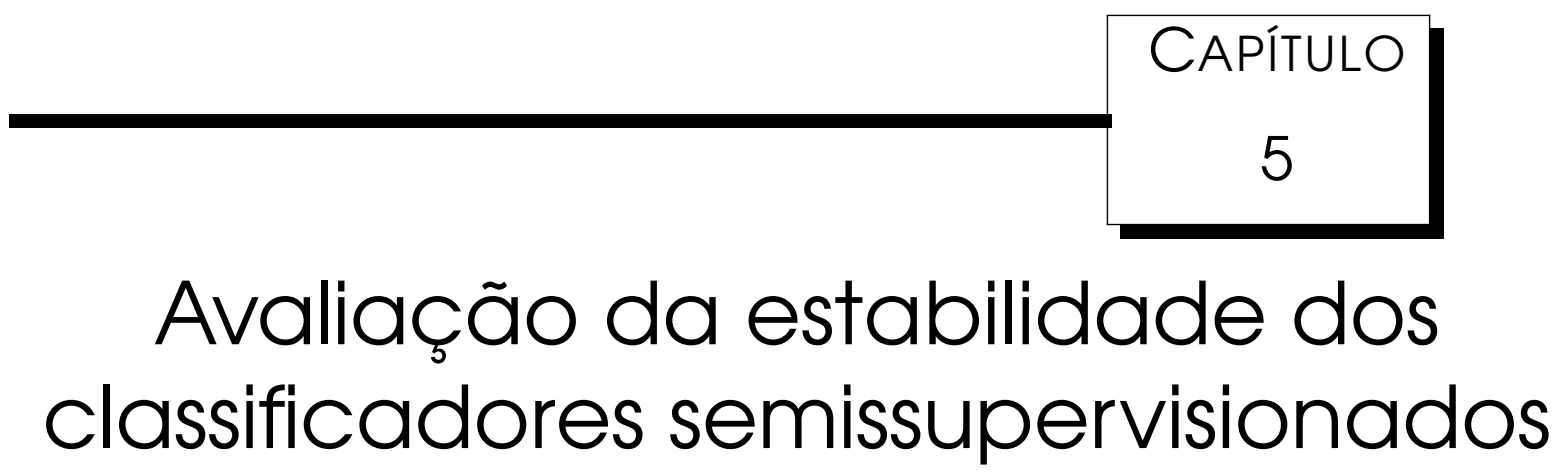

Neste capítulo, propõe-se um modelo de avaliação experimental que visa avaliar a estabilidade dos classificadores semissupervisionados usando uma variedade de métodos de geração de grafos com uma variedade de valores para o parâmetro de esparsificação $k$. Conforme já mencionado, classificadores que apresentam boa estabilidade podem ser preferiveis em relação à classificadores que apresentam excelente desempenho de classificação para apenas um pequeno subconjunto dos valores dos parâmetros. Portanto, o modelo de avaliação experimental proposto neste capítulo é uma ferramenta inestimável para verificar quais classificadores apresentam um bom balanço entre desempenho de classificação e estabilidade com respeito a um dado conjunto de valores do parâmetro de esparsificação.

Este capítulo está organizado como a seguir. Na Seção 5.1, descreve-se o modelo de avaliação da estabilidade dos classificadores semissupervisionados, proposto neste capítulo. Na Seção 5.2, discutem-se os resultados obtidos tendo como foco verificar o balanço entre desempenho de classificação e estabilidade dos algoritmos. Por fim, na Seção 5.3, faz-se as considerações finais deste capítulo.

\subsection{Descrição do modelo experimental proposto}

O intuito do modelo experimental proposto neste capítulo é analisar como que a taxa de erro dos classificadores é influenciada pelo valor do parâmetro de esparsificação. Em outras palavras, pretende-se avaliar quão estáveis são os classificadores semissupervisionados com respeito ao valor do parâmetro de esparsificação.

A avaliação da estabilidade dos classificadores semissupervisionados é feita fixando-se cada base de dados e cada método de geração de grafos. Com 
isso, pode-se avaliar a estabilidade dos classificadores semissupervisionados variando-se o valor do parâmetro de esparsificação $k$. As curvas de erro geradas pelos classificadores semissupervisionados são analisadas ao longo de todos os valores de $k$.

Como é inviável analisar de maneira combinatória a estabilidade dos classificadores dado todos os parâmetros, optou-se por fixar os parâmetros de regularização dos classificadores semissupervisionados. Para os classificadores que apresentam ao menos um parâmetro de regularização, fixam-se os valores dos parâmetros que obtiveram o melhor resultado na análise de melhor caso e então varia-se o valor do parâmetro $k$. Com isso, os algoritmos LGC, LapRLS e LapSVM são comparados com os demais classificadores usando bons valores para seus parâmetros de regularização.

Apesar de essa análise poder ser injusta com os algoritmos que são livres de parâmetros (GRF e RMGT), analisar a influência dos parâmetros de regularização combinados com o parâmetro de esparsificação é inviável devido à excessiva quantidade de resultados a serem gerados. Com isso, optou-se por realizar a avaliação dos parâmetros de regularização usando outro modelo experimental. Esse modelo é descrito no Capítulo 7 e visa avaliar a estabilidade das superfícies de erro geradas pelos classificadores semissupervisionados com respeito aos valores dos parâmetros de regularização.

Os resultados mostrados neste capítulo seguem o padrão mostrado na Figura 5.1. Os algoritmos GRF, LGC, LapRLS, LapSVM e RMGT são comparados empiricamente nessa avaliação. Os resultados são gerados a partir do uso do protocolo experimental descrito no Capítulo 3. Os resultados obtidos para essa avaliação são mostrados nas Figuras 5.2 até 5.19 e discutidos na Seção 5.2 .

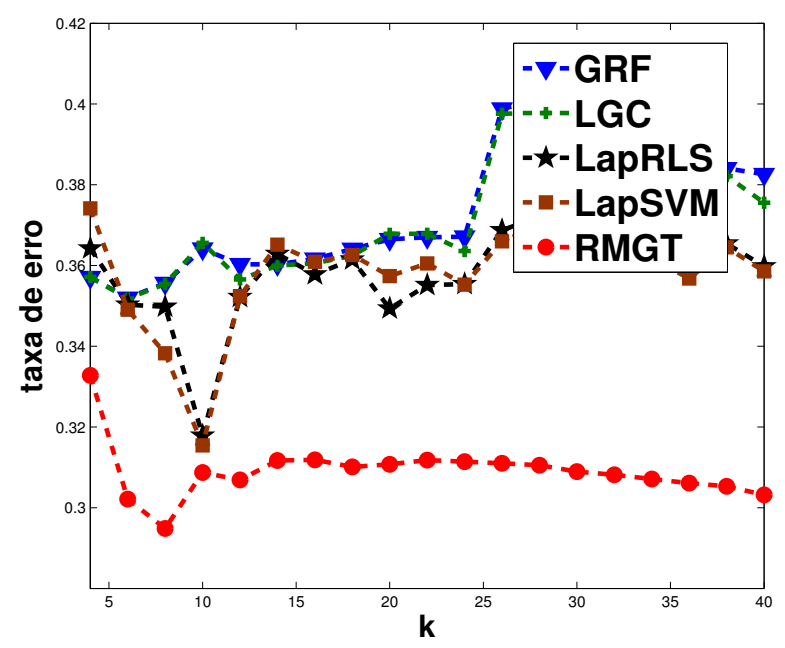

Figura 5.1: Padrão de gráfico para a avaliação da estabilidade dos classificadores semissupervisionados. 


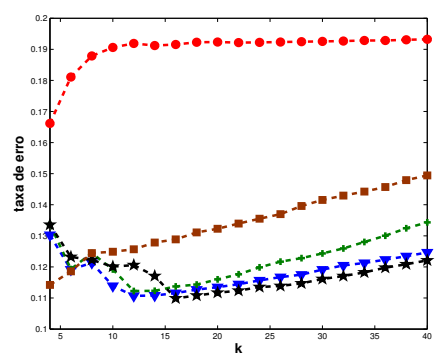

(a) USPS

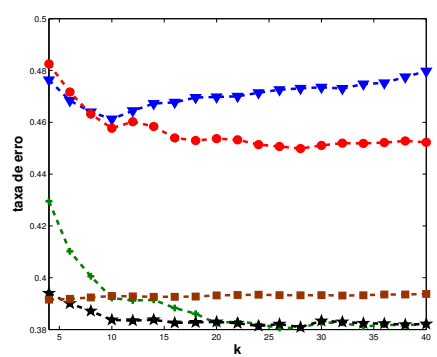

(d) G-241N

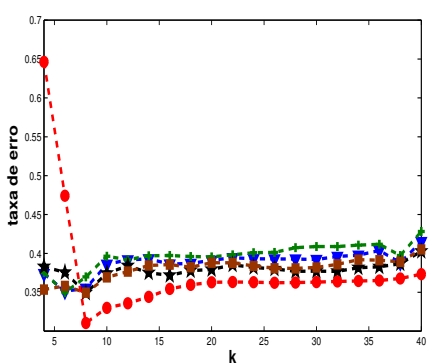

(b) $\mathrm{COIL}_{2}$

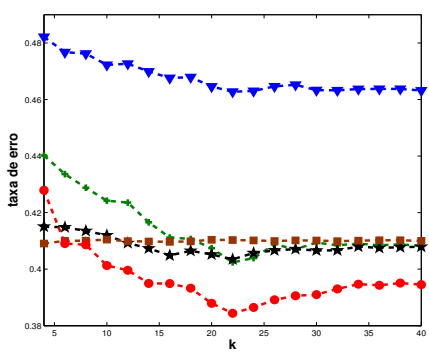

(e) G-241C

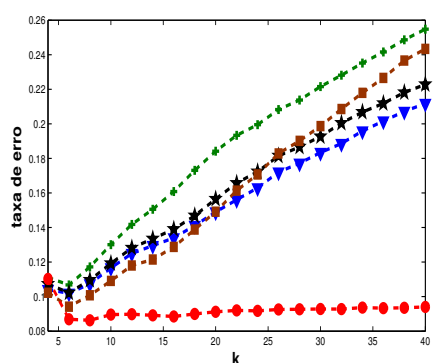

(c) DIGIT-1

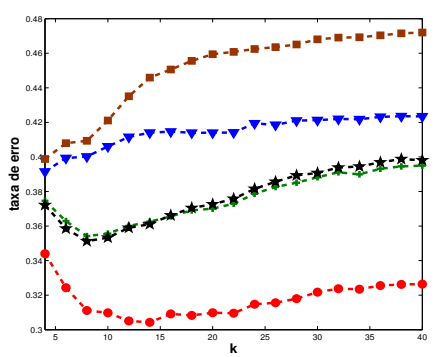

(f) TEXT

Figura 5.2: Estabilidade dos classificadores semissupervisionados para as partições de 10 exemplos rotulados usando o grafo symKNN-RBF.

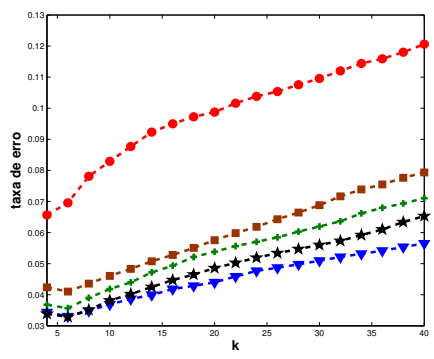

(a) USPS

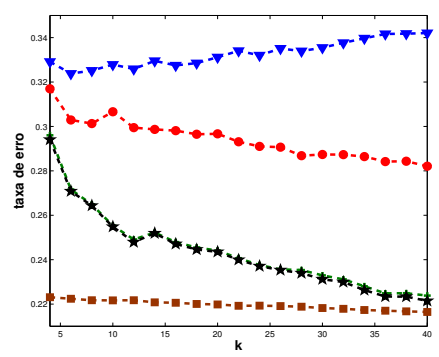

(d) $\mathrm{G}-241 \mathrm{~N}$

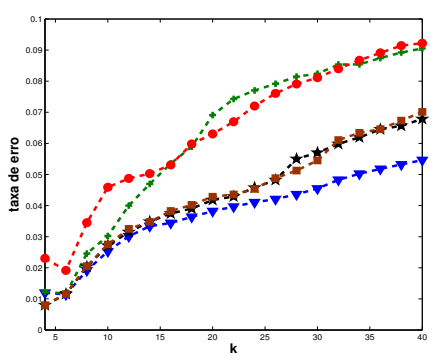

(b) $\mathrm{COIL}_{2}$

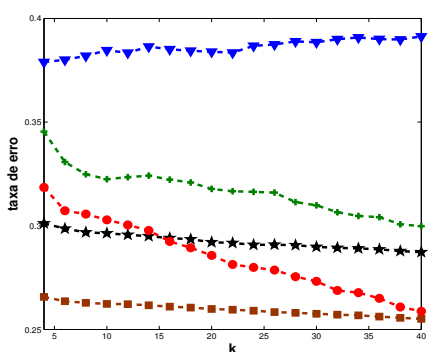

(e) G-241C

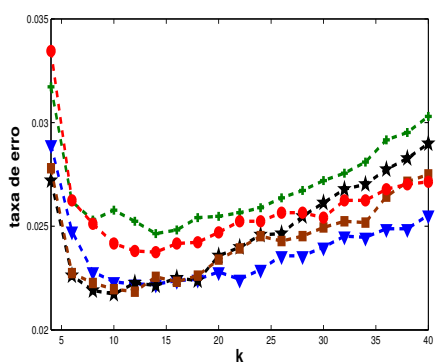

(c) DIGIT-1

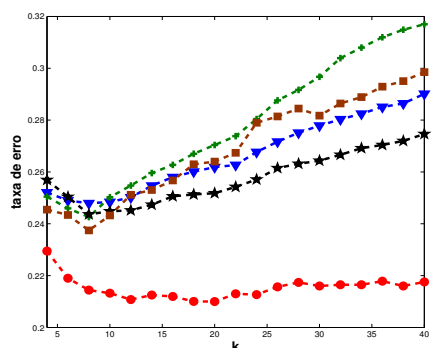

(f) TEXT

Figura 5.3: Estabilidade dos classificadores semissupervisionados para as partições de 100 exemplos rotulados usando o grafo symKNN-RBF. 


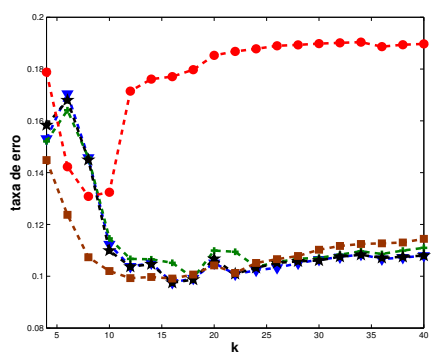

(a) USPS

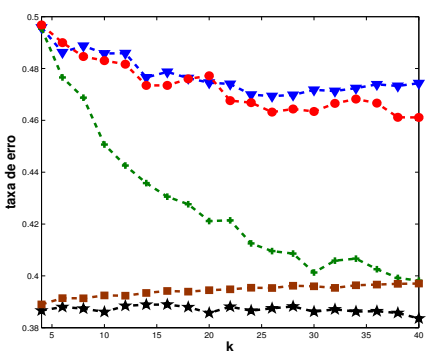

(d) $\mathrm{G}-241 \mathrm{~N}$

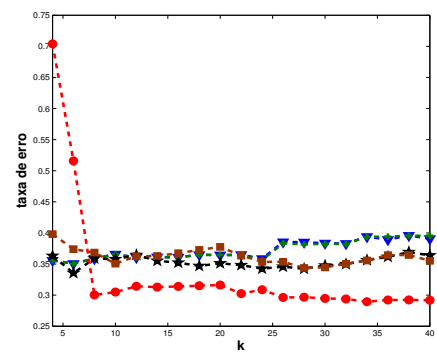

(b) $\mathrm{COIL}_{2}$

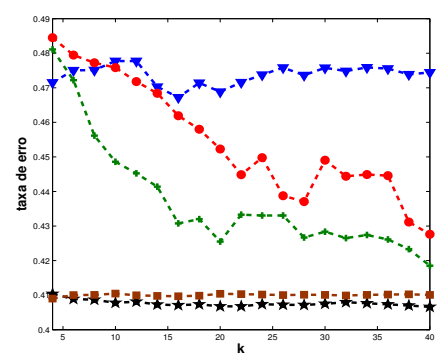

(e) G-241C

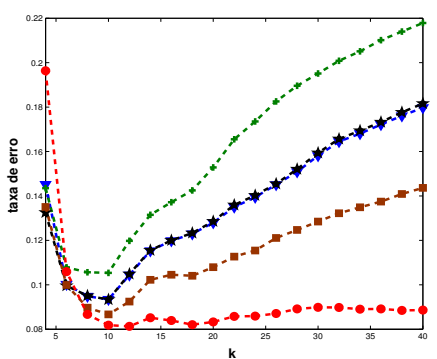

(c) DIGIT-1

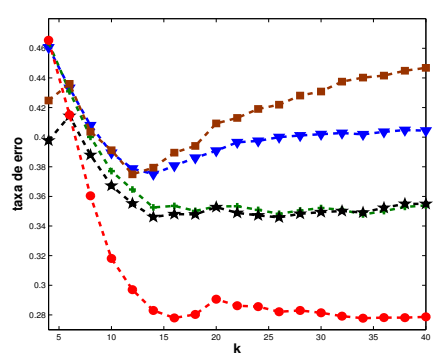

(f) TEXT

Figura 5.4: Estabilidade dos classificadores semissupervisionados para as partições de 10 exemplos rotulados usando o grafo mutKNN-RBF.

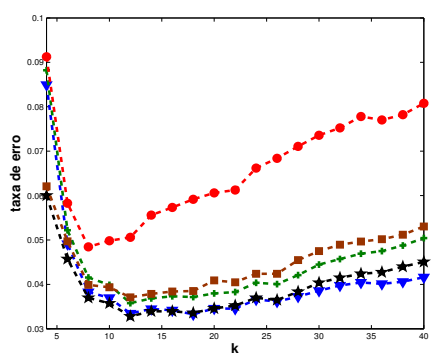

(a) USPS

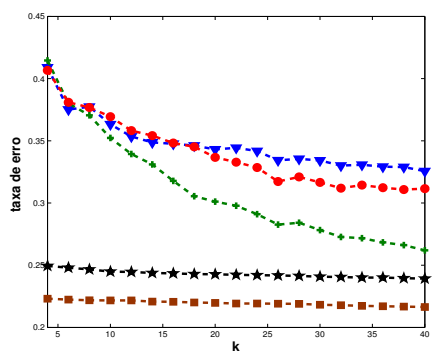

(d) $\mathrm{G}-241 \mathrm{~N}$

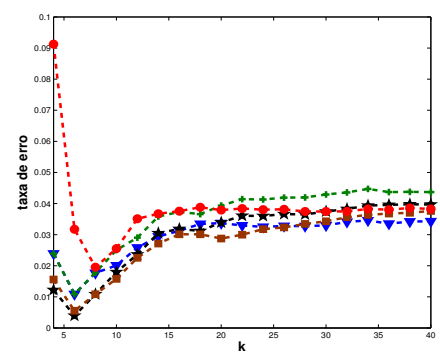

(b) $\mathrm{COIL}_{2}$

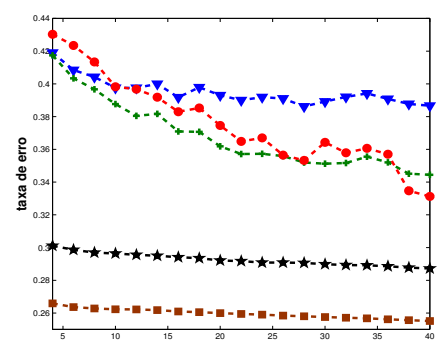

(e) G-241C

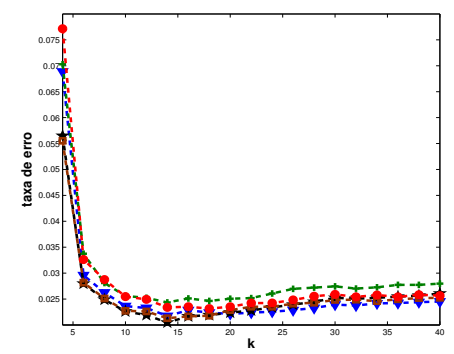

(c) DIGIT-1

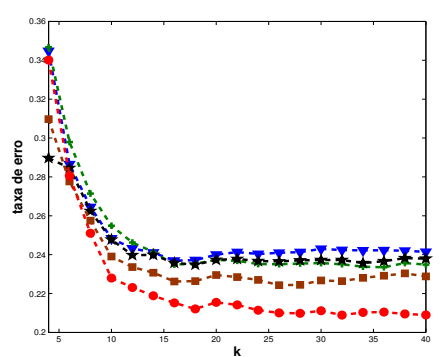

(f) TEXT

Figura 5.5: Estabilidade dos classificadores semissupervisionados para as partições de 100 exemplos rotulados usando o grafo mutKNN-RBF. 


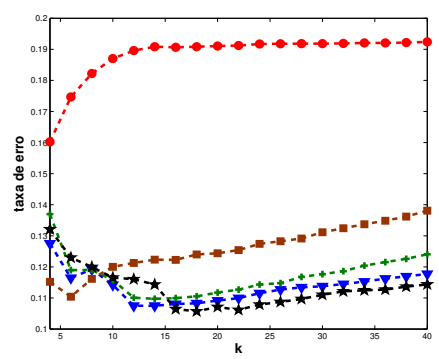

(a) USPS

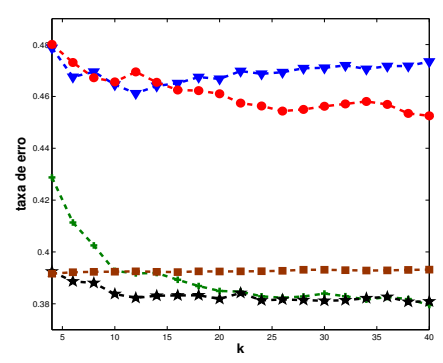

(d) $\mathrm{G}-241 \mathrm{~N}$

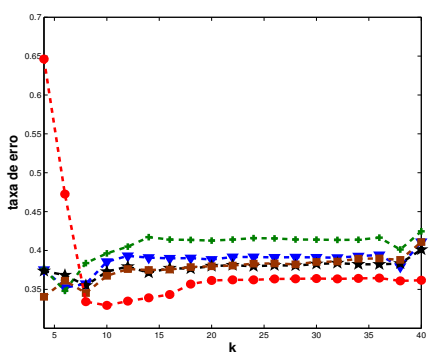

(b) $\mathrm{COIL}_{2}$

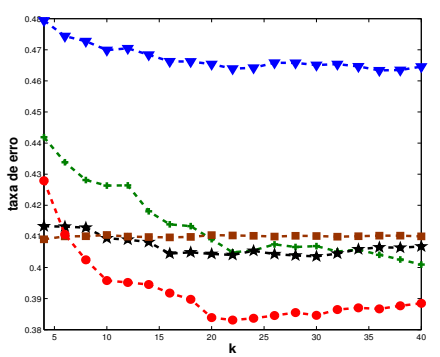

(e) G-241C

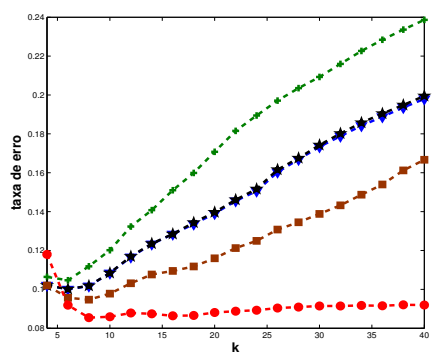

(c) DIGIT-1

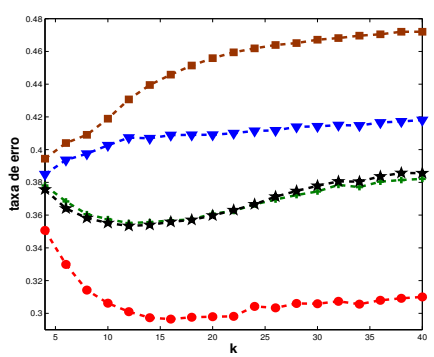

(f) TEXT

Figura 5.6: Estabilidade dos classificadores semissupervisionados para as partições de 10 exemplos rotulados usando o grafo symFKNN-RBF.

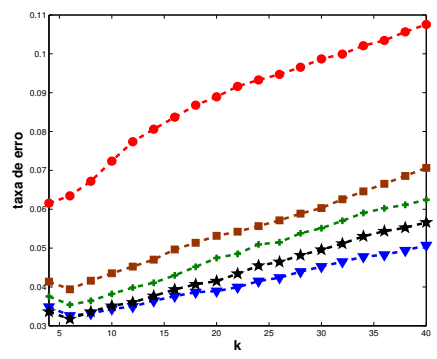

(a) USPS

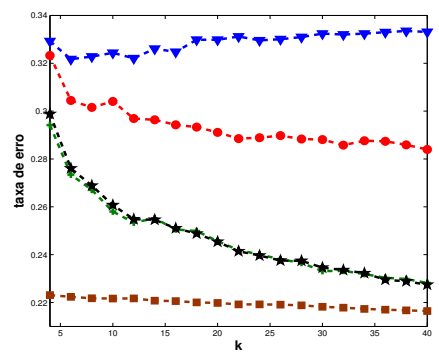

(d) $\mathrm{G}-241 \mathrm{~N}$

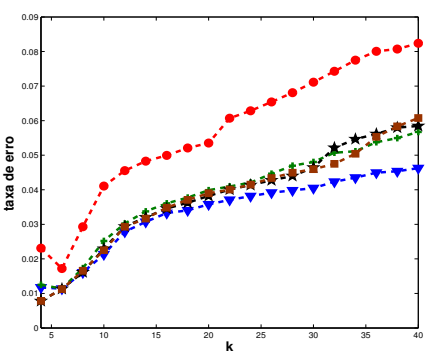

(b) $\mathrm{COIL}_{2}$

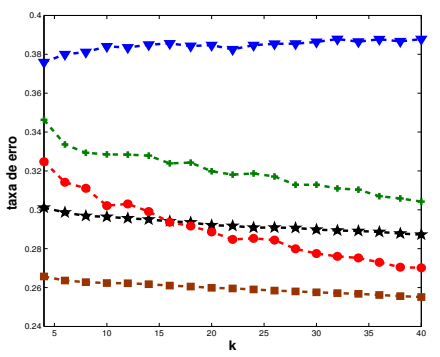

(e) G-241C

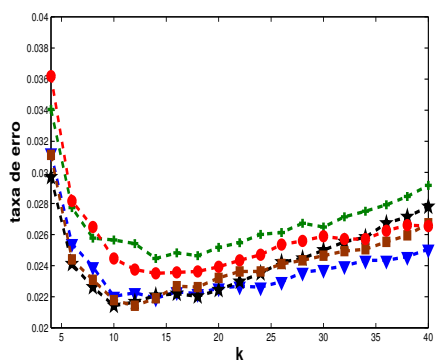

(c) DIGIT-1

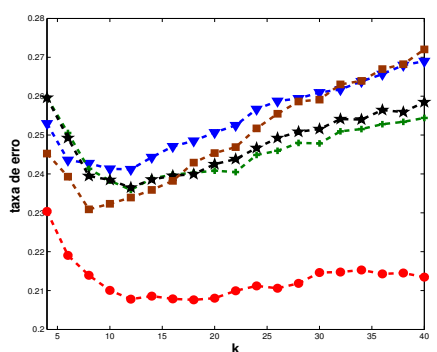

(f) TEXT

Figura 5.7: Estabilidade dos classificadores semissupervisionados para as partições de 100 exemplos rotulados usando o grafo symFKNN-RBF. 


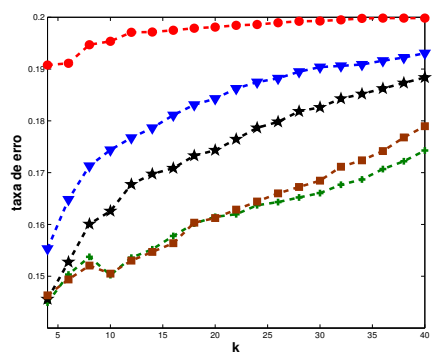

(a) USPS

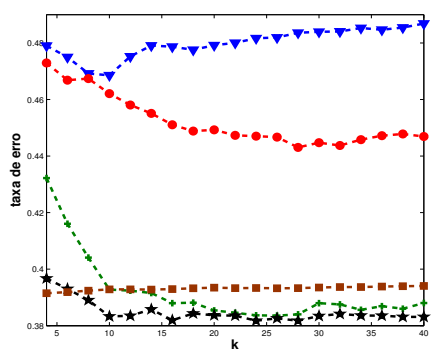

(d) $\mathrm{G}-241 \mathrm{~N}$

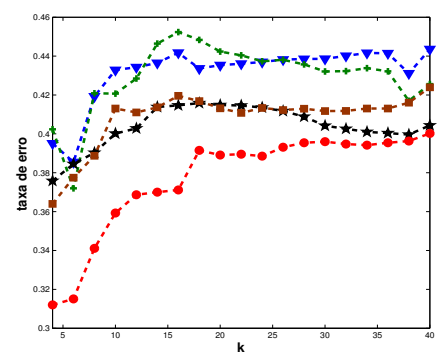

(b) $\mathrm{COIL}_{2}$

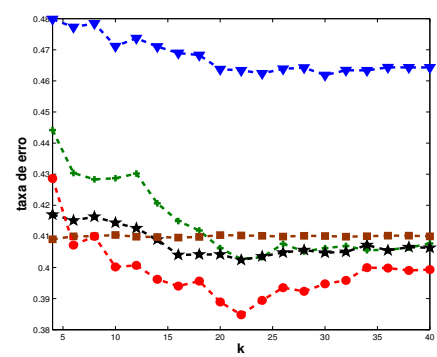

(e) G-241C

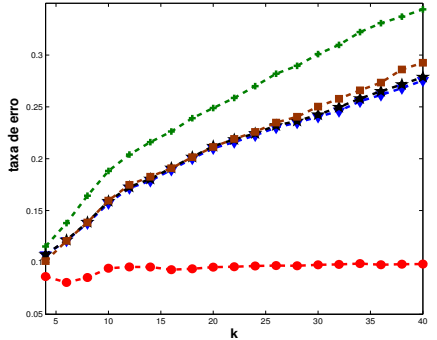

(c) DIGIT-1

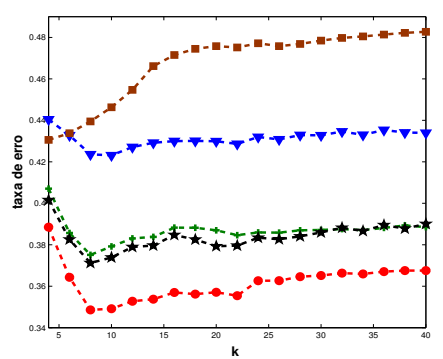

(f) TEXT

Figura 5.8: Estabilidade dos classificadores semissupervisionados para as partições de 10 exemplos rotulados usando o grafo symKNN-HM.

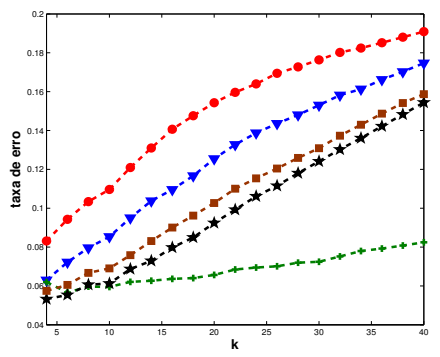

(a) USPS

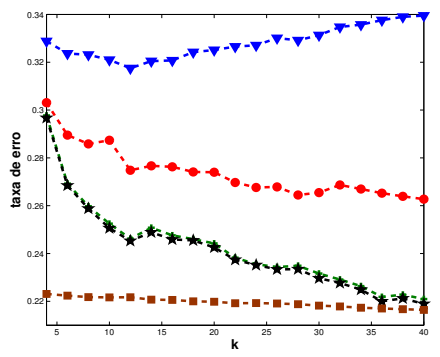

(d) G-241N

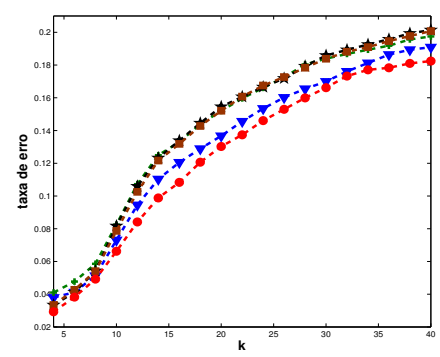

(b) $\mathrm{COIL}_{2}$

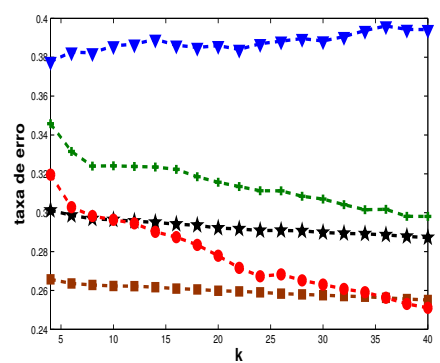

(e) G-241C

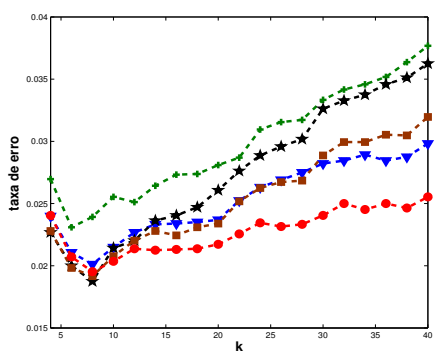

(c) DIGIT-1

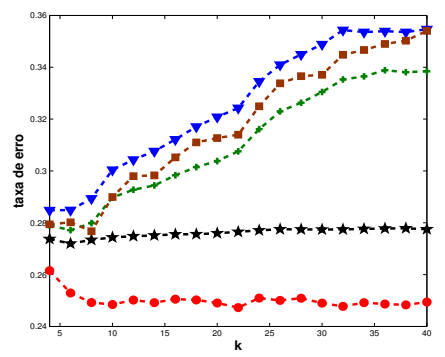

(f) TEXT

Figura 5.9: Estabilidade dos classificadores semissupervisionados para as partições de 100 exemplos rotulados usando o grafo symKNN-HM. 


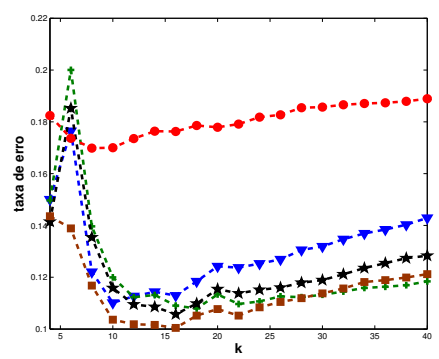

(a) USPS

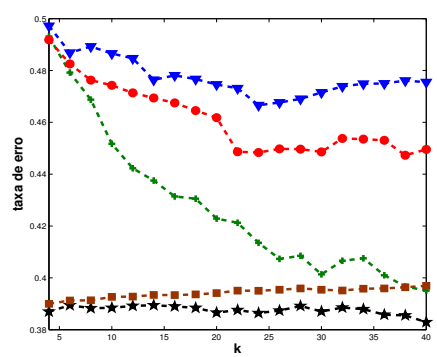

(d) $\mathrm{G}-241 \mathrm{~N}$

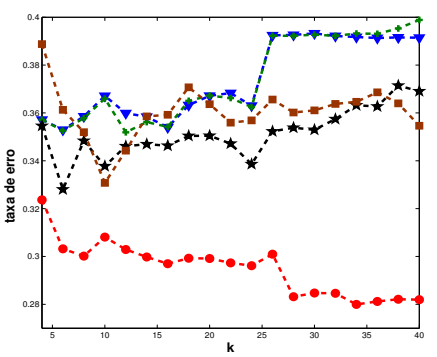

(b) $\mathrm{COIL}_{2}$

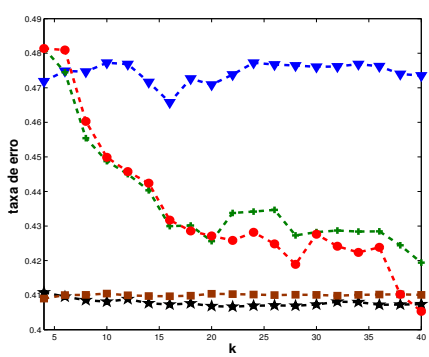

(e) G-241C

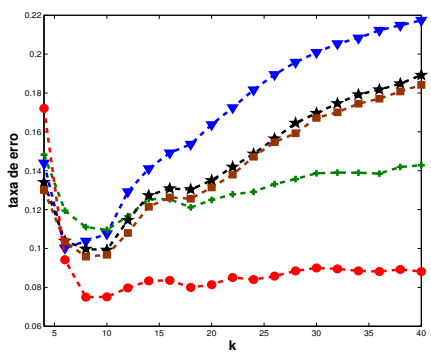

(c) DIGIT-1

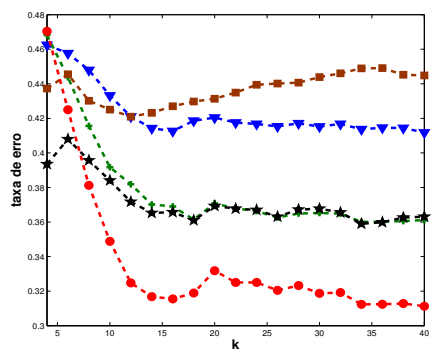

(f) TEXT

Figura 5.10: Estabilidade dos classificadores semissupervisionados para as partições de 10 exemplos rotulados usando o grafo mutKNN-HM.

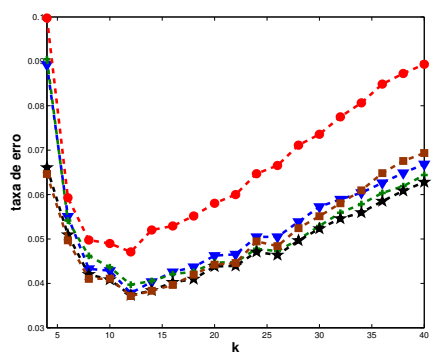

(a) USPS

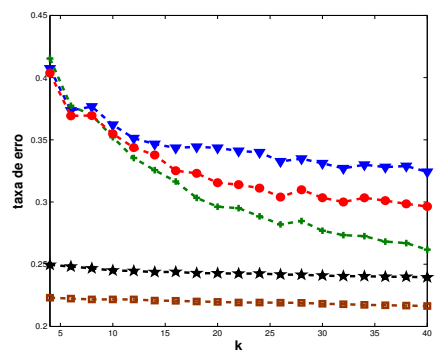

(d) $\mathrm{G}-241 \mathrm{~N}$

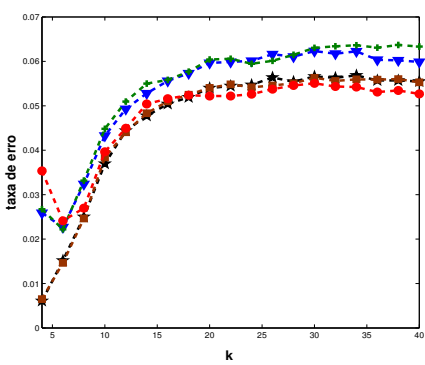

(b) $\mathrm{COIL}_{2}$

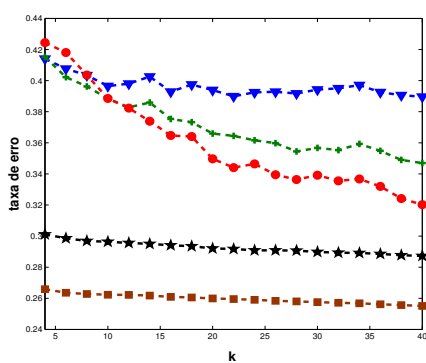

(e) G-241C

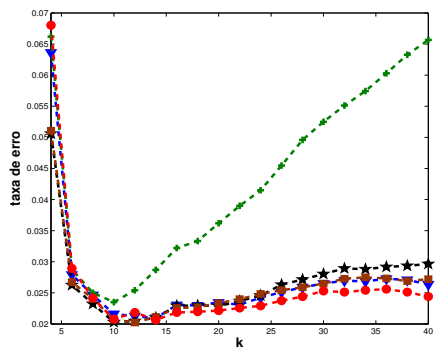

(c) DIGIT-1

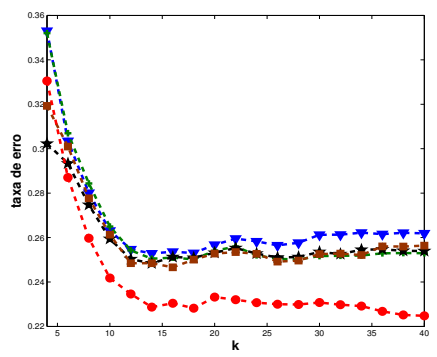

(f) TEXT

Figura 5.11: Estabilidade dos classificadores semissupervisionados para as partições de 100 exemplos rotulados usando o grafo mutKNN-HM. 


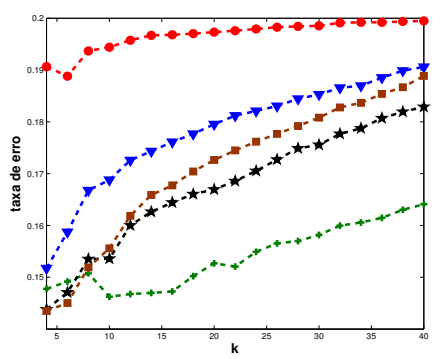

(a) USPS

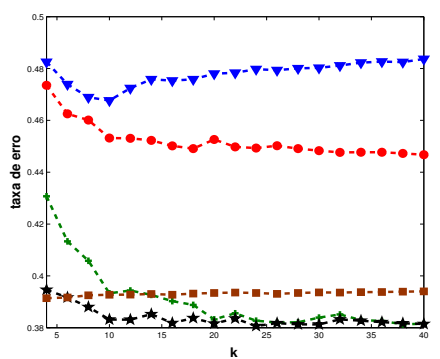

(d) $\mathrm{G}-241 \mathrm{~N}$

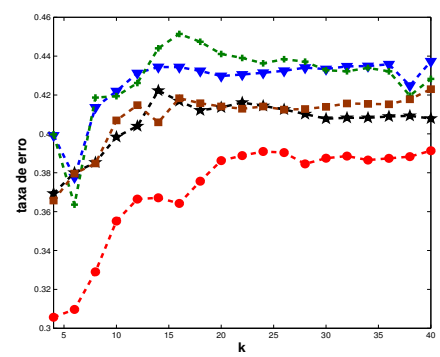

(b) $\mathrm{COIL}_{2}$

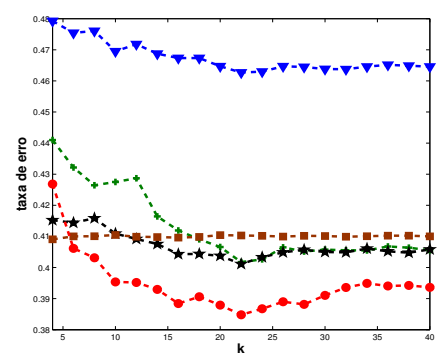

(e) G-241C

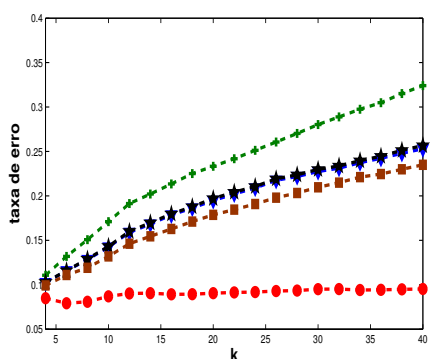

(c) DIGIT-1

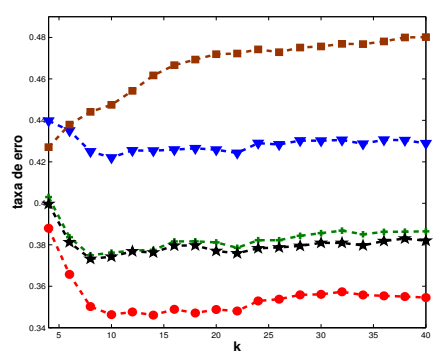

(f) TEXT

Figura 5.12: Estabilidade dos classificadores semissupervisionados para as partições de 10 exemplos rotulados usando o grafo symFKNN-HM.

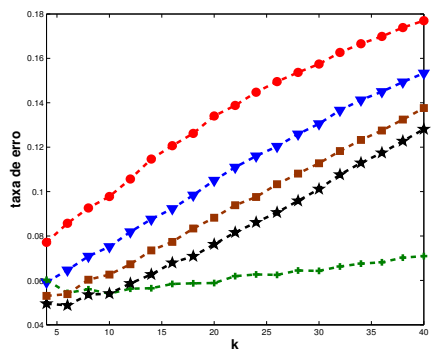

(a) USPS

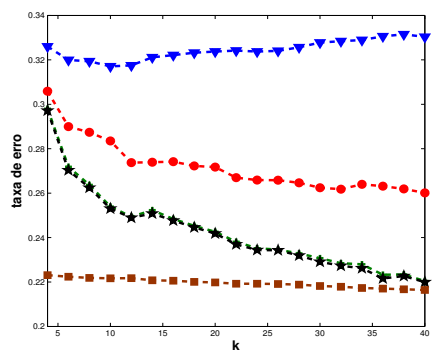

(d) G-241N

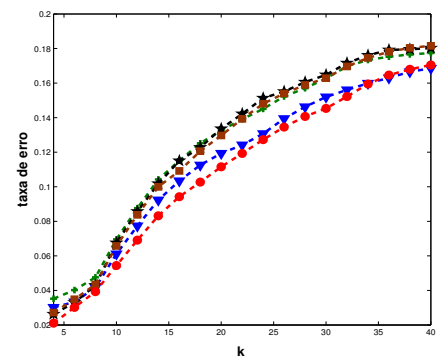

(b) $\mathrm{COIL}_{2}$

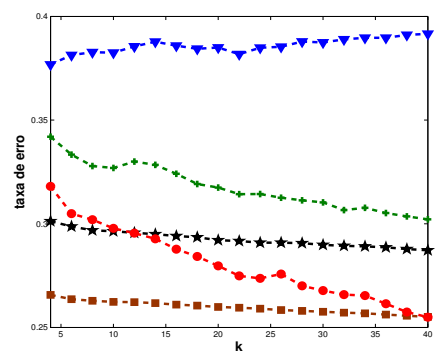

(e) G-241C

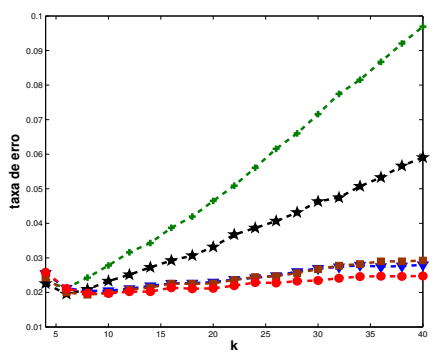

(c) DIGIT-1

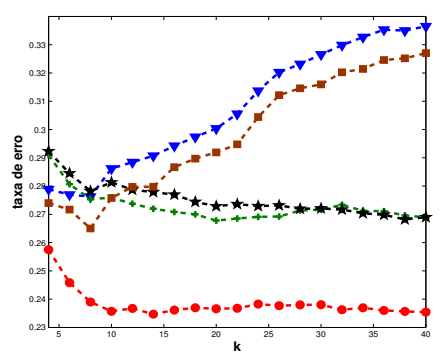

(f) TEXT

Figura 5.13: Estabilidade dos classificadores semissupervisionados para as partições de 100 exemplos rotulados usando o grafo symFKNN-HM. 


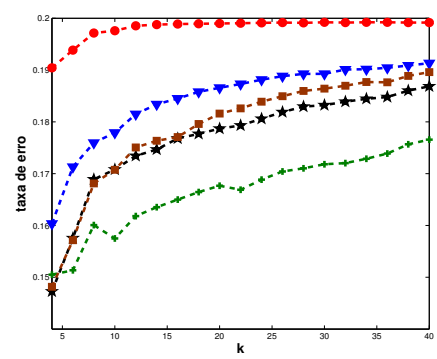

(a) USPS

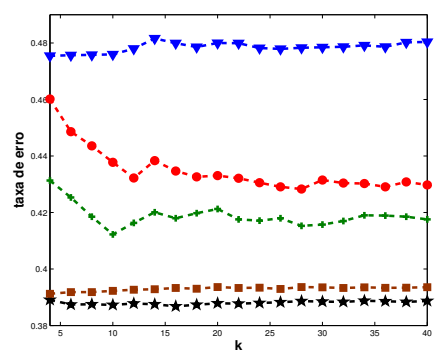

(d) $\mathrm{G}-241 \mathrm{~N}$

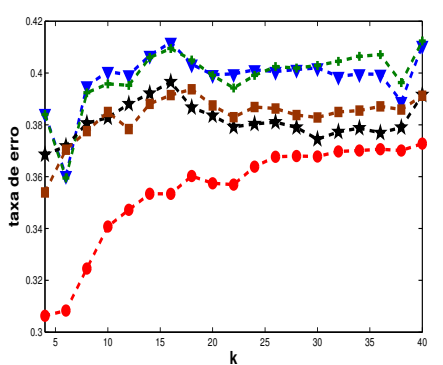

(b) $\mathrm{COIL}_{2}$

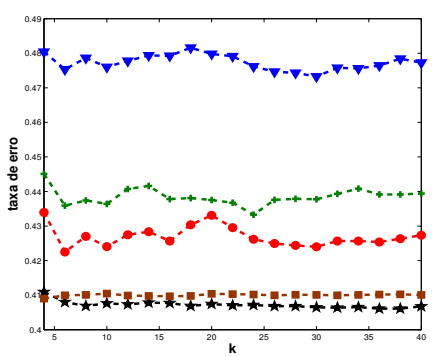

(e) G-241C

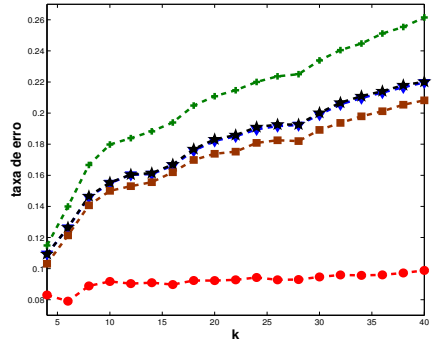

(c) DIGIT-1

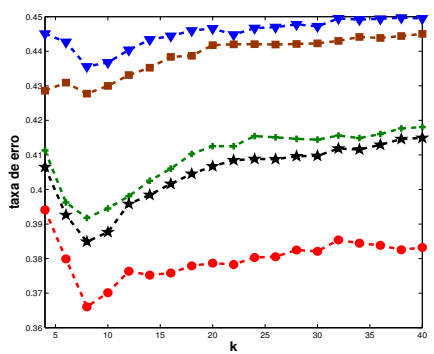

(f) TEXT

Figura 5.14: Estabilidade dos classificadores semissupervisionados para as partições de 10 exemplos rotulados usando o grafo symKNN-LLE.

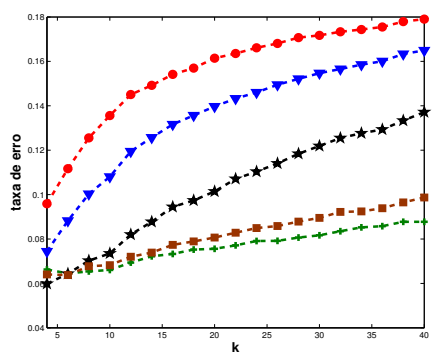

(a) USPS

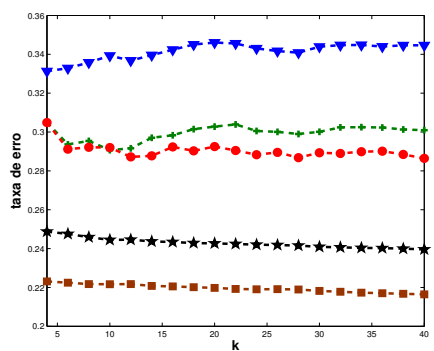

(d) $\mathrm{G}-241 \mathrm{~N}$

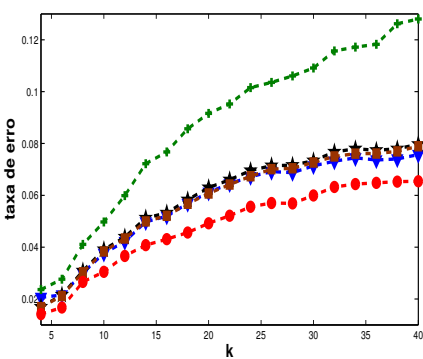

(b) $\mathrm{COIL}_{2}$

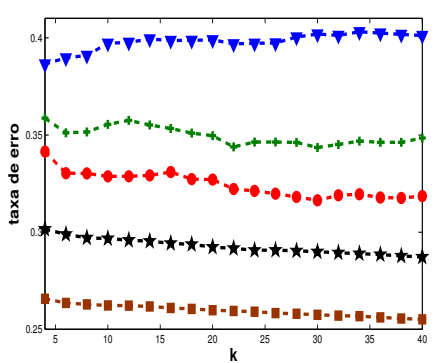

(e) G-241C

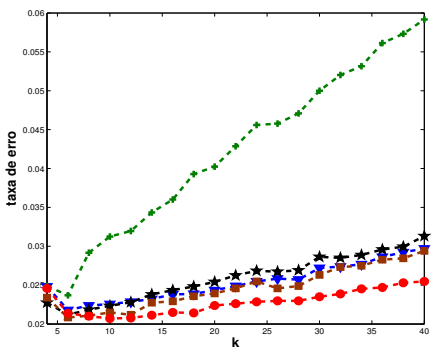

(c) DIGIT-1

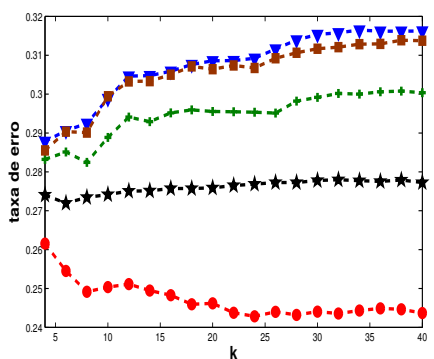

(f) TEXT

Figura 5.15: Estabilidade dos classificadores semissupervisionados para as partições de 100 exemplos rotulados usando o grafo symKNN-LLE. 


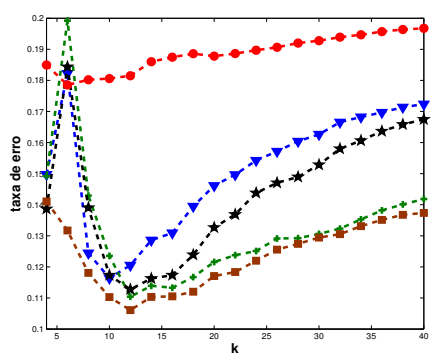

(a) USPS

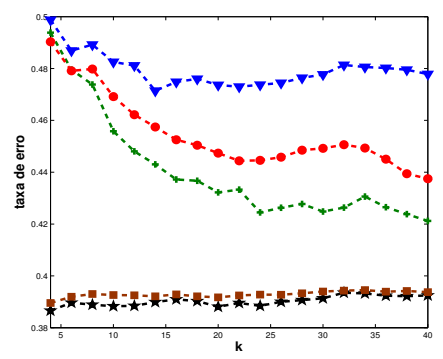

(d) $\mathrm{G}-241 \mathrm{~N}$

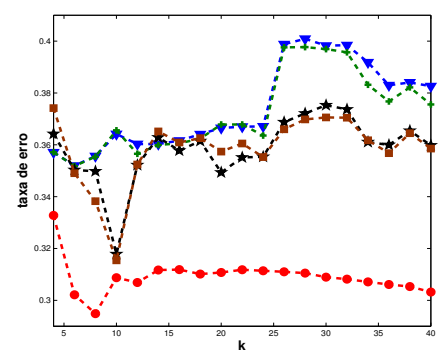

(b) $\mathrm{COIL}_{2}$

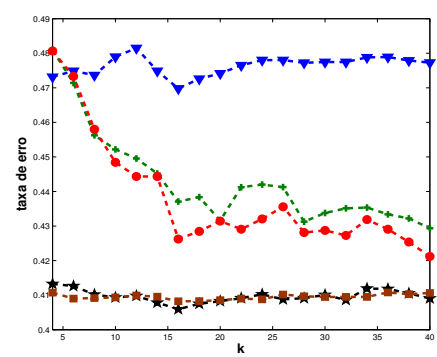

(e) G-241C

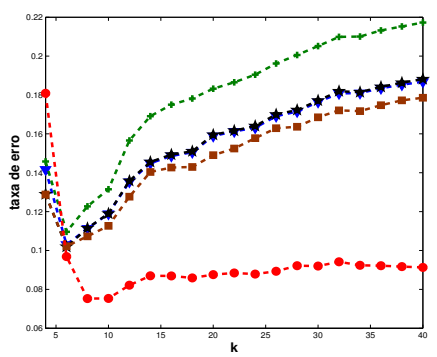

(c) DIGIT-1

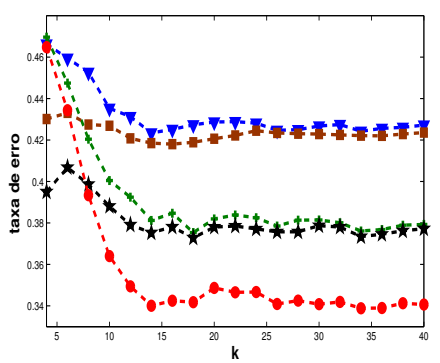

(f) TEXT

Figura 5.16: Estabilidade dos classificadores semissupervisionados para as partições de 10 exemplos rotulados usando o grafo mutKNN-LLE.

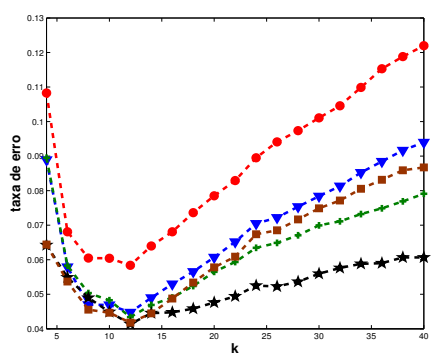

(a) USPS

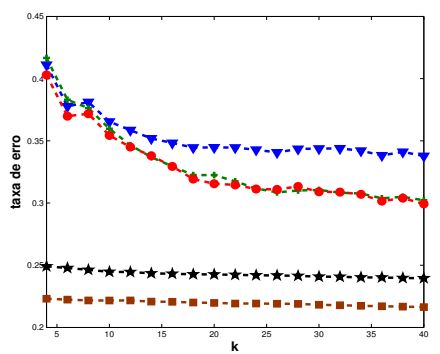

(d) G-241N

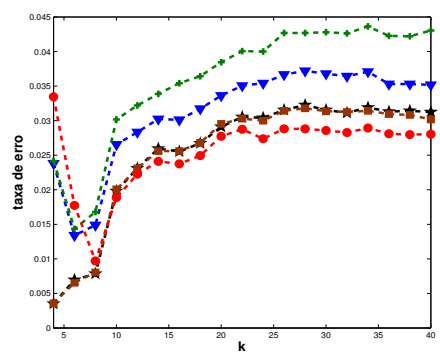

(b) $\mathrm{COIL}_{2}$

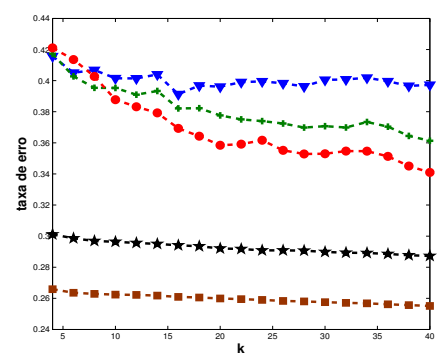

(e) G-241C

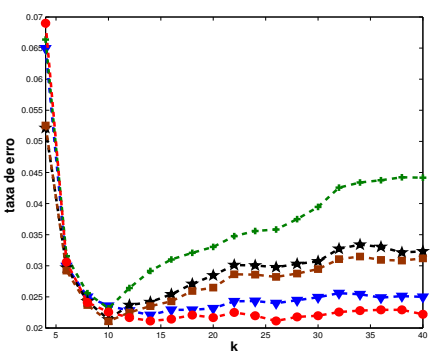

(c) DIGIT-1

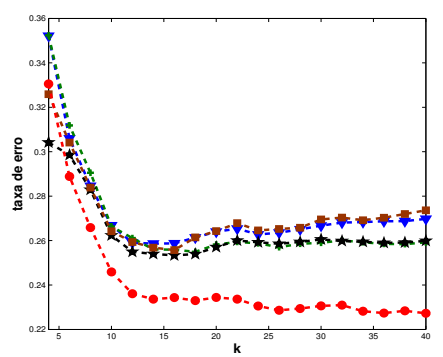

(f) TEXT

Figura 5.17: Estabilidade dos classificadores semissupervisionados para as partições de 100 exemplos rotulados usando o grafo mutKNN-LLE. 


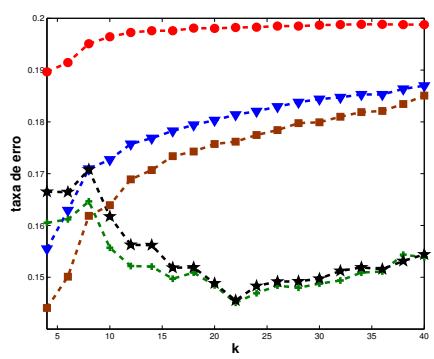

(a) USPS

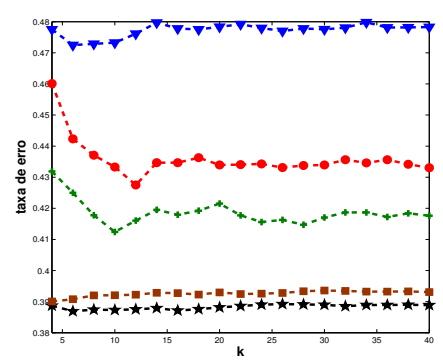

(d) $\mathrm{G}-241 \mathrm{~N}$

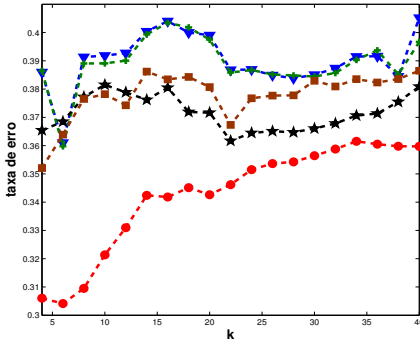

(b) $\mathrm{COIL}_{2}$

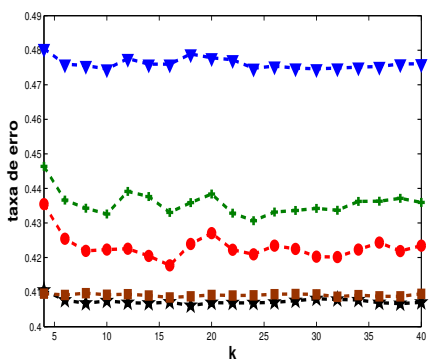

(e) G-241C

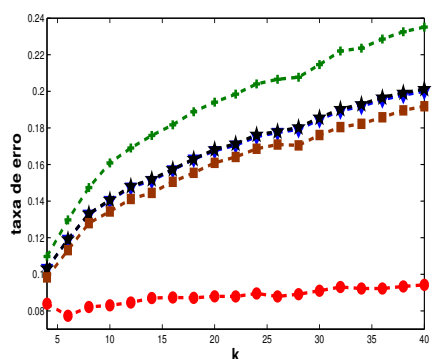

(c) DIGIT-1

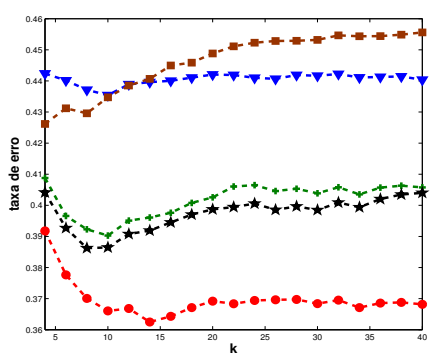

(f) TEXT

Figura 5.18: Estabilidade dos classificadores semissupervisionados para as partições de 10 exemplos rotulados usando o grafo symFKNN-LLE.

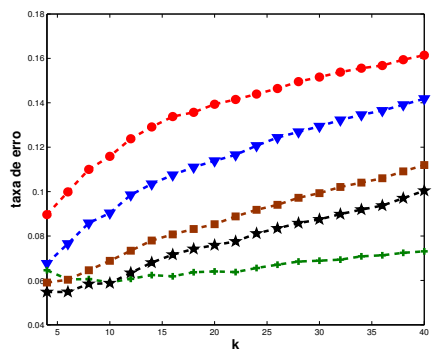

(a) USPS

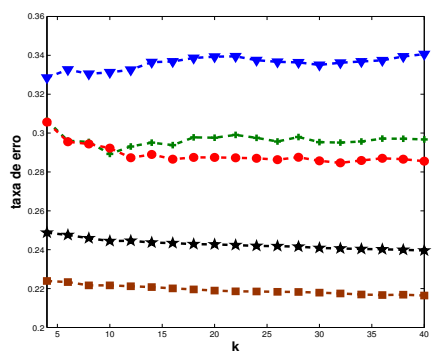

(d) $\mathrm{G}-241 \mathrm{~N}$

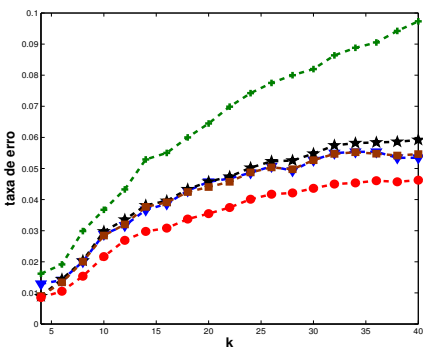

(b) $\mathrm{COIL}_{2}$

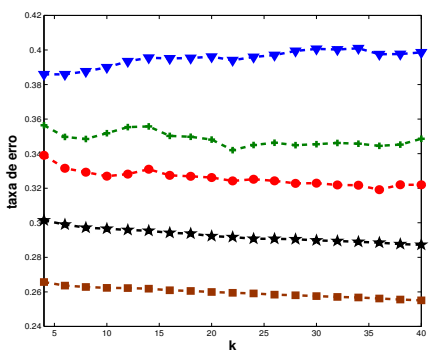

(e) G-241C

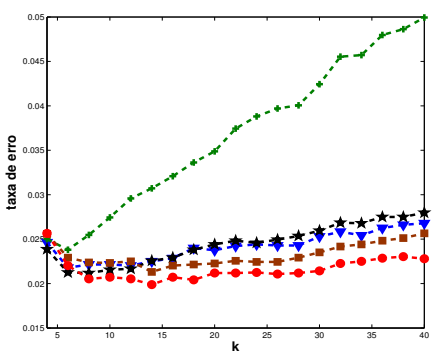

(c) DIGIT-1

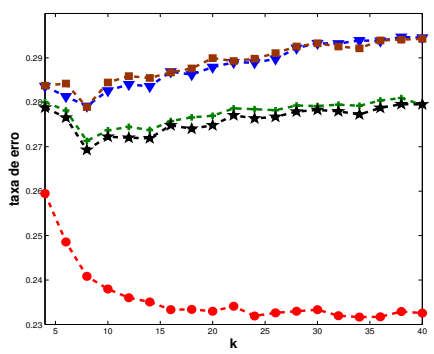

(f) TEXT

Figura 5.19: Estabilidade dos classificadores semissupervisionados para as partições de 100 exemplos rotulados usando o grafo symFKNN-LLE. 


\subsection{Avaliação dos resultados obtidos}

A partir da análise dos resultados obtidos, percebe-se as seguintes constatações empíricas:

- Para todos os métodos de geração de grafos e partições, o algoritmo LapSVM apresentou excelente estabilidade nas bases G-241N e G-241C, além de um bom desempenho de classificação;

- Para todos os métodos de geração de grafos e partições, o algoritmo LapRLS apresentou excelente estabilidade na base G-241C, além de um bom desempenho de classificação;

- O algoritmo RMGT apresentou elevada instabilidade nas partições de 10 exemplos rotulados para $k \in\{4,6\}$ ao se usar o kernel RBF na base $\mathrm{COIL}_{2}$, conforme mostrado nas Figuras 5.2, 5.4 e 5.6;

- Para todos os métodos de geração de grafos nas partições de 10 exemplos rotulados, o algoritmo RMGT apresentou excelentes desempenho de classificação e estabilidade na base DIGIT-1, enquanto que os demais classificadores não apresentaram resultados satisfatórios;

- Nas partições de 100 exemplos rotulados, o algoritmo RMGT apresentou uma elevada instabilidade na base $\mathrm{COIL}_{2}$ ao se usar o grafo mutKNN com $k \in\{4,6\}$, conforme mostrado nas Figuras 5.5, 5.11 e 5.17. A instabilidade desse algoritmo pode ser considerada pequena apenas ao se usar o grafo mutKNN-HM, conforme mostrado na Figura 5.11;

- Para todas as partições, todos os classificadores apresentaram alta instabilidade nas bases DIGIT-1 e TEXT ao se usar o grafo mutKNN com valores relativamente pequenos de $k$, conforme mostrado nas Figuras $5.4,5.5,5.10,5.11,5.16$ e 5.17 ;

- Para as partições de 100 exemplos rotulados e todos os métodos de geração de grafos, os resultados para os algoritmos LGC e RMGT nas bases G-241N e G-241C usando grafos densos são melhores que os resultados para esses algoritmos usando grafos esparsos. Isso indica que a premissa de que grafos esparsos tendem a gerar melhores resultados que grafos densos, amplamente usada na literatura de aprendizado semissupervisionado (Zhu, 2005), pode não ser sempre válida.

- Para todas as partições e todos os métodos de geração de grafos, o algoritmo GRF apresentou alta estabilidade nas bases G-241N e G-241C. Entretanto, os resultados para esse classificador não são competitivos com os resultados dos demais classificadores;

- Para as partições de 10 exemplos rotulados e todos os métodos de geração de grafos, nota-se que o algoritmo RMGT não é competitivo com os demais classificadores na base USPS. Ao se usar o grafo mutKNN, há várias 
trocas nos rankings dos classificadores, o que pode dar margem à elaboração de trabalhos empíricos "maliciosos". Para exemplificar, tomam-se como exemplo as duas argumentações descritas a seguir, tendo com base a Figura 5.4.

Argumentação 1 Grafos esparsos tendem a gerar melhores resultados experimentais que grafos densos (Zhu, 2005). Como o grafo mutKNN-RBF obteve o melhor ranking médio na análise de melhor caso nas partições de 10 exemplos rotulados, conforme mostrado na Tabela 4.2, esse grafo fora usado na avaliação experimental deste trabalho. Para a avaliação experimental, escolheu-se $k=6$, conforme sugerido por Liu e Chang (2009). A partir dos resultados obtidos, nota-se que o algoritmo RMGT obteve a segunda posição no ranking dos classificadores, perdendo apenas para o algoritmo LapSVM por aproximadamente $2 \%$ de taxa de erro. Como o algoritmo LapSVM possui dois parâmetros de regularização e o processo de escolha de parâmetros pode afetar drasticamente o desempenho de classificação dos classificadores semissupervisionados, o algoritmo LapSVM pode não ser efetivo em aplicações reais. Com isso, deve-se optar pelo uso do algoritmo RMGT em aplicações reais tendo em vista que ele é livre de parâmetros, além de apresentar um bom desempenho de classificação.

Argumentação 2 Como o grafo mutKNN-RBF obteve o melhor ranking médio na análise de melhor caso nas partições de 10 exemplos rotulados, conforme mostrado na Tabela 4.2, esse grafo fora usado na avaliação experimental deste trabalho. O grafo mutKNN pode ser instável para valores relativamente pequenos de $k$ devido ao fato da possivel existência de diversos componentes desconexos no grafo. Isso faria com que a Premissa 1 não fosse satisfeita e poderia reduzir drasticamente o desempenho de classificação dos classificadores semissupervisionados. Como mostrado na Figura 5.3, para as bases G-241N e G-241C, a premissa de que grafos esparsos geram melhores resultados que grafos densos (Zhu, 2005) pode não ser sempre válida. Portanto, escolheu-se usar neste trabalho um valor de $k$ para o qual o grafo gerado seja conexo. Essa configuração faria com que a Premissa 1 fosse satisfeita. Especificamente, escolheu-se $k=25$, que é uma das sugestões de Hein e Maier (2007). A partir dos resultados obtidos, conclui-se que o algoritmo RMGT não é competitivo com os demais classificadores. Como o algoritmo GRF ficou em segundo lugar no ranking dos classificadores e é livre de parâmetros, esse algoritmo deve ser o escolhido para se usar em aplicações reais.

Os Argumentos 1 e 2 mostram como diferentes conclusões podem ser obtidas a partir de protocolos experimentais enviesados, mesmo com duas argumentações válidas, tendo como embasamento evidências experimentais da literatura. Logo, a avaliação da estabilidade dos classificadores semissupervisionados, proposta neste trabalho, é de fundamental importância para evitar conclusões enviesadas; 
- Na Figura 5.2, nota-se que o algoritmo RMGT obteve um bom desempenho de classificação e uma boa estabilidade nas bases $\mathrm{COIL}_{2}$, DIGIT-1, G-241C e TEXT. Percebe-se ainda uma elevada estabilidade dos algoritmos LGC, LapRLS e LapSVM nas bases USPS, COIL 2 , G-241N e G-241C;

- Na Figura 5.3, nota-se um comportamento similar dos algoritmos GRF, LGC, LapRLS e LapSVM nas bases USPS, $\mathrm{COIL}_{2}$, DIGIT-1 e TEXT;

- Na Figura 5.4, nota-se uma alta estabilidade dos algoritmos LapSVM e LapRLS nas bases $\mathrm{COIL}_{2}$, G-241N e G-241C. O algoritmo RMGT apresentou um excelente desempenho de classificação e estabilidade nas bases $\mathrm{COIL}_{2}$, DIGIT-1 e TEXT para a maioria dos valores de $k$;

- Na Figura 5.5, nota-se que todos os classificadores obtiveram comportamentos similares nas bases $\mathrm{COIL}_{2}$, DIGIT-1 e TEXT;

- Na Figura 5.6, nota-se que todos os classificadores apresentaram boa estabilidade nas bases G-241N e G-241C. Todos os classificadores, com exceção do algoritmo LapSVM, apresentaram boa estabilidade na base TEXT. O algoritmo RMGT apresentou a melhor estabilidade na base DIGIT1 ;

- Na Figura 5.7, nota-se que o algoritmo RMGT apresentou o melhor desempenho de classificação e a melhor estabilidade na base TEXT;

- Na Figura 5.8, nota-se que todos os classificadores, com exceção do algoritmo LapSVM, apresentaram boa estabilidade na base TEXT;

- Na Figura 5.9, nota-se que o algoritmo LapSVM é o único classificador estável na base G-241N. Na base USPS, o algoritmo LGC é o único classificador estável, enquanto que os algoritmos LapRLS e RMGT são os únicos classificadores estáveis na base TEXT;

- Na Figura 5.10, nota-se que todos os classificadores apresentaram alta instabilidade na base TEXT para valores relativamente pequenos de $k$, enquanto que a estabilidade de todos os classificadores é evidenciada nessa base quando $k \geq 16$. Na base USPS, chega-se ao mesmo problema discutido nas Argumentações 1 e 2. O algoritmo RMGT apresentou o melhor desempenho de classificação e a melhor estabilidade nas bases $\mathrm{COIL}_{2}$ e DIGIT-1;

- Na Figura 5.11, percebe-se a alta instabilidade do algoritmo LGC na base DIGIT-1. Para as demais bases de dados, os classificadores tiveram comportamentos similares;

- Na Figura 5.12, nota-se que todos os classificadores, com exceção do algoritmo LapSVM, obtiveram alta estabilidade na base TEXT. Os algoritmos LGC, LapRLS e LapSVM apresentaram bons desempenho de classificação e estabilidade na base G-241N. O algoritmo RMGT apresentou os melhores resultados nas bases $\mathrm{COIL}_{2}$, DIGIT-1, G-241C e TEXT; 
- Na Figura 5.13, nota-se que o algoritmo LapSVM foi o único algoritmo estável na base G-241N. Os algoritmos LGC e RMGT obtiveram boa estabilidade na base G-241C. Na base TEXT, os algoritmos LGC, LapRLS e RMGT apresentaram boa estabilidade, sendo que o algoritmo RMGT obteve o melhor desempenho de classificação. Os algoritmos GRF, LapSVM e RMGT obtiveram uma boa estabilidade na base DIGIT-1, enquanto que o algoritmo LGC obteve uma boa estabilidade na base USPS;

- Na Figura 5.14, percebe-se que o algoritmo RMGT não é competitivo com os demais classificadores na base USPS. Todos os classificadores apresentaram boa estabilidade nas bases G-241C e G-241N;

- Na Figura 5.15, nota-se que os algoritmos LGC, LapRLS e RMGT apresentaram boa estabilidade na base TEXT. Todos os classificadores, com exceção do algoritmo LGC, apresentaram boa estabilidade na base DIGIT-1. $\mathrm{O}$ algoritmo LGC apresentou uma certa instabilidade na base $\mathrm{COIL}_{2}$. Os algoritmos LGC e LapSVM apresentaram boa estabilidade na base USPS;

- Na Figura 5.16, nota-se que apenas os algoritmos LapRLS e LapSVM obtiveram alta estabilidade nas bases G-241N e G-241C. Os algoritmos LGC e RMGT apresentaram alta instabilidade na base TEXT para $k \leq 14$. Entretanto, todos os classificadores apresentaram boa estabilidade nessa base para $k \geq 16$. O algoritmo RMGT apresentou alta estabilidade nas bases $\mathrm{COIL}_{2}$ e DIGIT-1 para $k \geq 8$;

- Na Figura 5.17, nota-se que todos os classificadores apresentaram comportamentos similares em todas as bases de dados, com exceção das bases G-241N e G-241C;

- Na Figura 5.18, nota-se que todos os classificadores obtiveram boa estabilidade nas bases G-241N, G-241C e TEXT;

- Na Figura 5.19, nota-se uma instabilidade do algoritmo LGC nas bases $\mathrm{COIL}_{2}$ e DIGIT-1. Entretanto, o algoritmo LGC apresentou elevada estabilidade na base USPS. Todos os classificadores obtiveram boa estabilidade nas bases G-241N, G-241C e TEXT.

\subsection{Considerações finais}

Neste capítulo, propôs-se um modelo de avaliação experimental para avaliar a estabilidade de classificadores semissupervisionados baseados em grafos. A partir da análise dos resultados, constatou-se que há uma alta variância no ranking dos classificadores, o que dificulta tirar conclusões gerais. Alguns padrões foram encontrados, tal como o excelente desempenho de classificação e a alta estabilidade do algoritmo RMGT nas bases $\mathrm{COIL}_{2}$, DIGIT-1 e TEXT para a maioria dos valores de $k$. Entretanto, vários resultados apresentam pontos negativos para a área de aprendizado semissupervisionado, conforme constatado, por exemplo, nas Argumentações 1 e 2. 
Acredita-se que o modelo de avaliação experimental proposto neste capítulo deva ser amplamente usado na literatura de aprendizado semissupervisionado. Com o uso desse modelo de avaliação experimental, a quantidade de conclusões enviesadas referentes à avaliação de classificadores semissupervisionados seria drasticamente reduzida, o que aumentaria a maturidade empírica da área de aprendizado semissupervisionado. Ainda mais, acreditase que o uso do modelo proposto neste capítulo possa melhorar a qualidade dos trabalhos empíricos na área de aprendizado de máquina. 
CAPÍTULO

\section{Avaliação da influência da geração de grafos na classificação semissupervisionada}

Neste capítulo, propõe-se um modelo de avaliação experimental que visa avaliar a estabilidade dos métodos de geração de grafos usando uma variedade de valores para o parâmetro de esparsificação $k$ para cada classificador semissupervisionado. Em outras palavras, procura-se avaliar como os grafos gerados influenciam o desempenho de classificação dos classificadores semissupervisionados. Portanto, o modelo de avaliação experimental proposto neste capítulo é uma ferramenta inestimável para verificar quais métodos de geração de grafos apresentam um bom balanço entre desempenho de classificação e estabilidade com respeito a um dado conjunto de valores do parâmetro de esparsificação. Prentende-se ainda verificar quais grafos seriam os mais adequados para um determinado classificador semissupervisionado e uma determinada base de dados.

Este capítulo está organizado como a seguir. Na Seção 6.1, descreve-se o modelo de avaliação da influência da geração de grafos na classificação semissupervisionada, proposto neste capítulo. Na Seção 6.2, discutem-se os resultados obtidos tendo como foco verificar o balanço entre desempenho de classificação e estabilidade dos algoritmos. Por fim, na Seção 6.3, descrevemse as considerações finais deste capítulo.

\subsection{Descrição do modelo experimental proposto}

O intuito dessa avaliação experimental é avaliar (1) como os métodos de geração de grafos afetam o desempenho de classificação dos algoritmos de aprendizado semissupervisionado e (2) a estabilidade dos métodos de geração de grafos com respeito à variação dos valores do parâmetro de esparsifica- 
ção. Em outras palavras, pretende-se avaliar quão estáveis são os métodos de geração de grafos com respeito ao valor do parâmetro de esparsificação.

A avaliação da influência da geração de grafos na classificação semissupervisionada é feita fixando-se cada base de dados e cada classificador semissupervisionado. Com isso, pode-se avaliar a estabilidade dos métodos de geração de grafos variando-se o valor do parâmetro de esparsificação $k$. As curvas de erro geradas pelos métodos de geração de grafos são analisadas ao longo de todos os valores de $k$.

Como é inviável analisar de maneira combinatória a estabilidade dos métodos de geração de grafos dado todos os parâmetros, optou-se por fixar os parâmetros de regularização dos classificadores semissupervisionados, conforme discutido no Capítulo 5. Para os classificadores que apresentam ao menos um parâmetro de regularização, fixam-se os valores dos parâmetros que obtiveram o melhor resultado na análise de melhor caso e então variase o valor do parâmetro $k$. Com isso, os algoritmos LGC, LapRLS e LapSVM são avaliados com os demais classificadores usando bons valores para seus parâmetros de regularização.

Os resultados apresentados neste capítulo seguem o padrão mostrado na Figura 6.1. Os grafos symKNN-RBF, mutKNN-RBF, symFKNN-RBF, symKNNHM, mutKNN-HM, symFKNN-HM, symKNN-LLE, mutKNN-LLE e symFKNNLLE são comparados empiricamente nesta avaliação. Os resultados são gerados a partir do uso do protocolo experimental descrito no Capítulo 3. Os resultados obtidos para essa avaliação são mostrados nas Figuras 6.2 até 6.13 e discutidos na Seção 6.2.

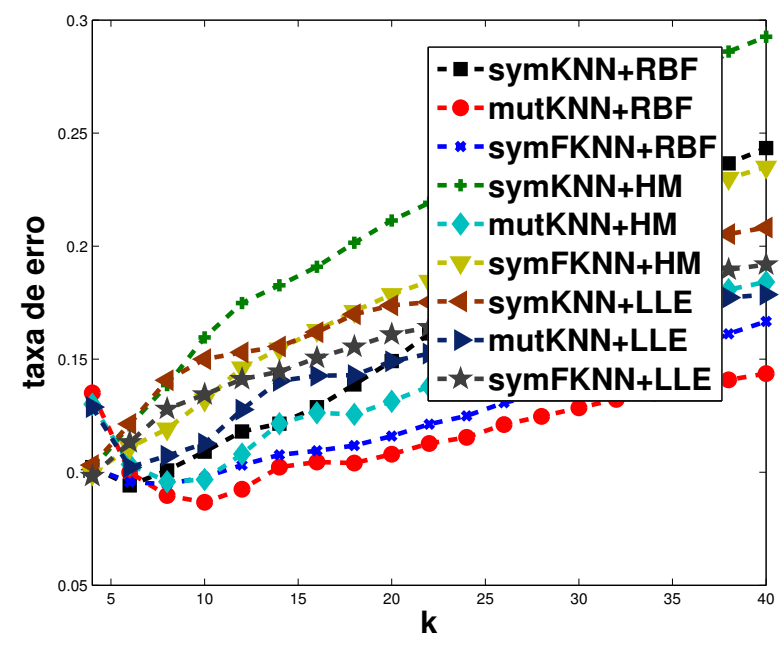

Figura 6.1: Padrão de gráfico para a avaliação da influência da geração de grafos na classificação semissupervisionada. 


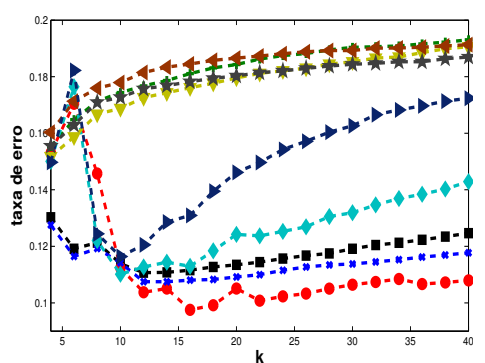

(a) GRF

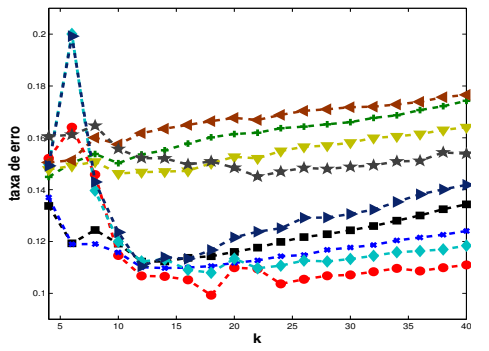

(b) LGC

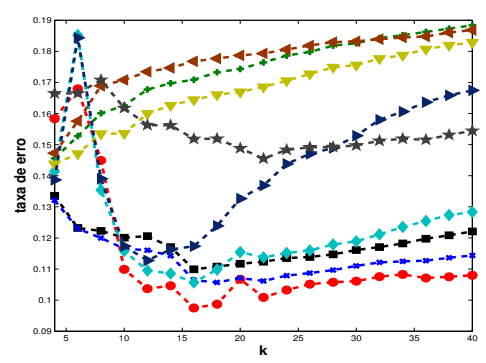

(c) LapRLS

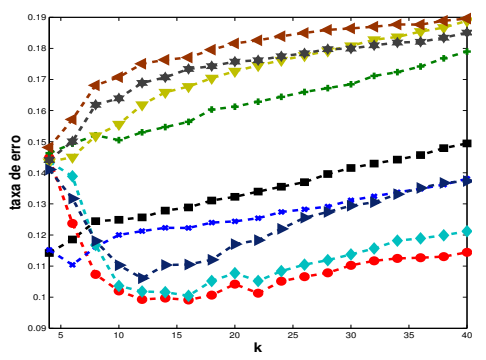

(d) LapSVM

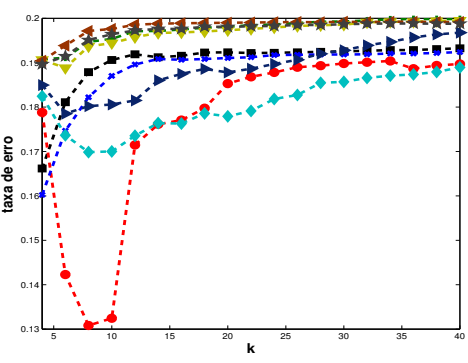

(e) RMGT

Figura 6.2: Avaliação dos métodos de geração de grafos na base de dados USPS usando as partições de 10 exemplos rotulados.

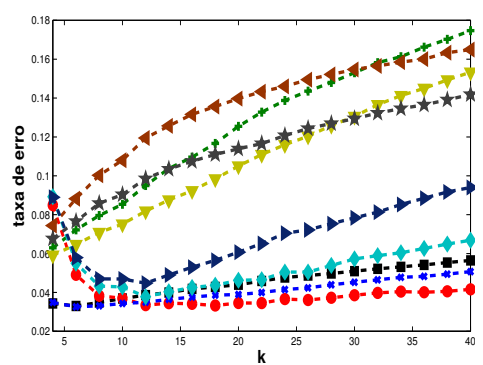

(a) GRF

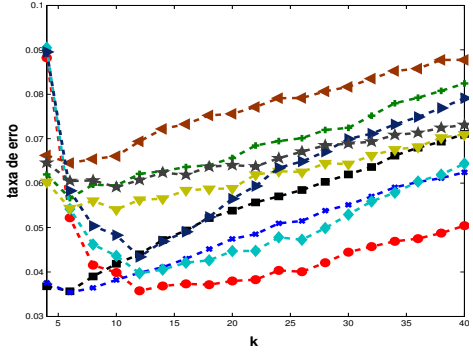

(b) LGC

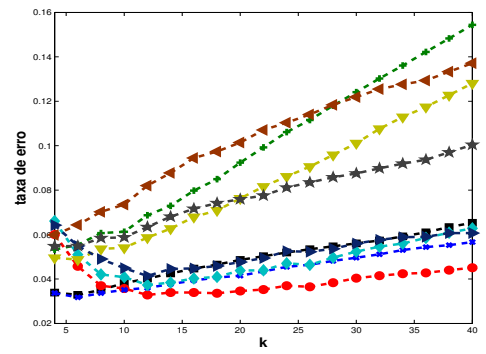

(c) LapRLS

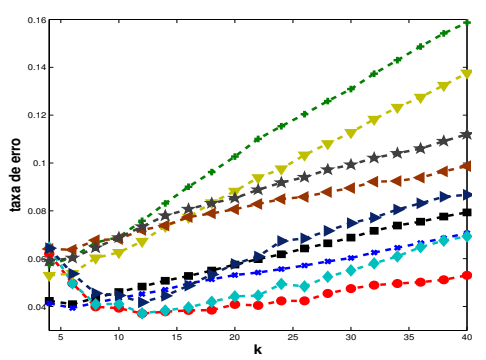

(d) LapSVM

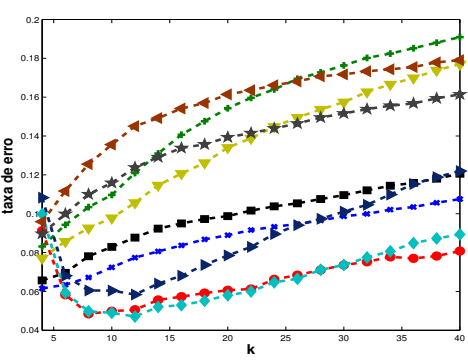

(e) RMGT

Figura 6.3: Avaliação dos métodos de geração de grafos na base de dados USPS usando as partições de 100 exemplos rotulados. 


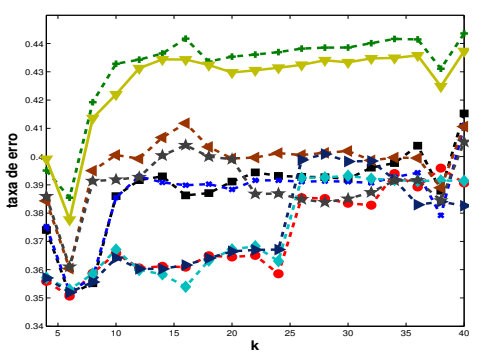

(a) GRF

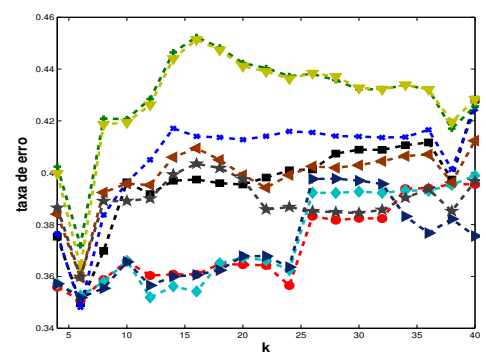

(b) LGC

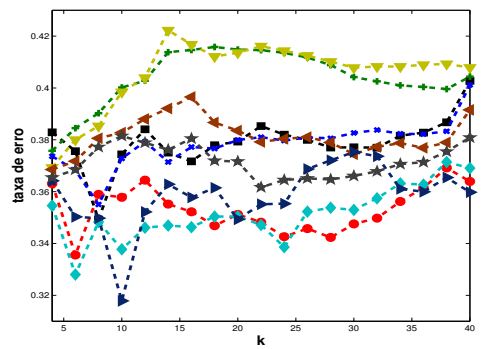

(c) LapRLS

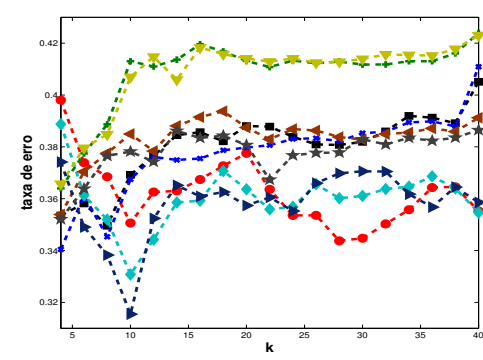

(d) LapSVM

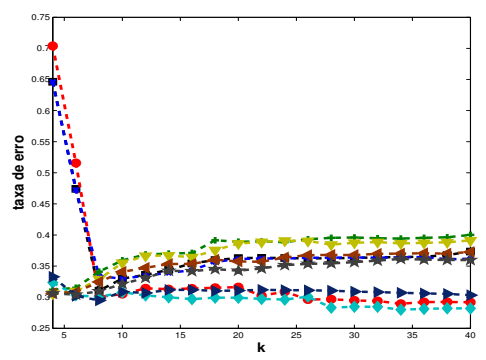

(e) RMGT

Figura 6.4: Avaliação dos métodos de geração de grafos na base de dados $\mathrm{COIL}_{2}$ usando as partições de 10 exemplos rotulados.

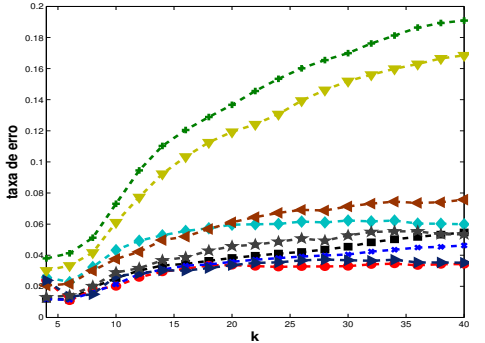

(a) GRF

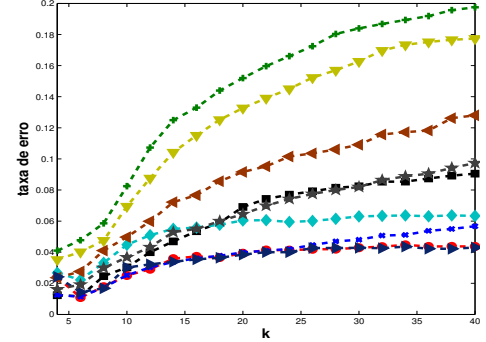

(b) LGC

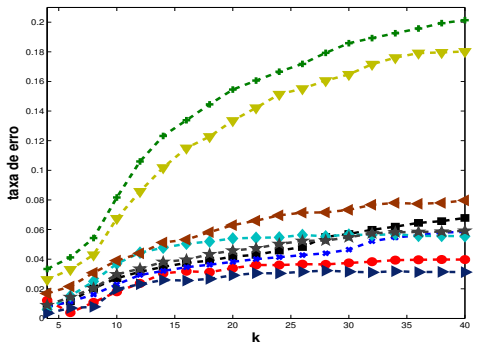

(c) LapRLS

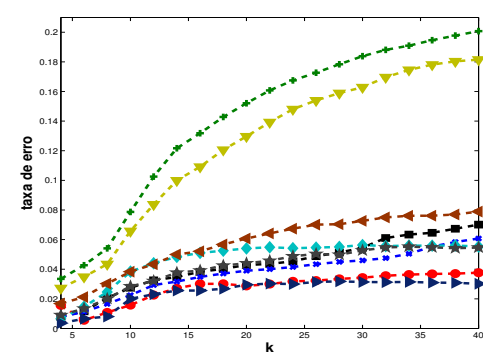

(d) LapSVM

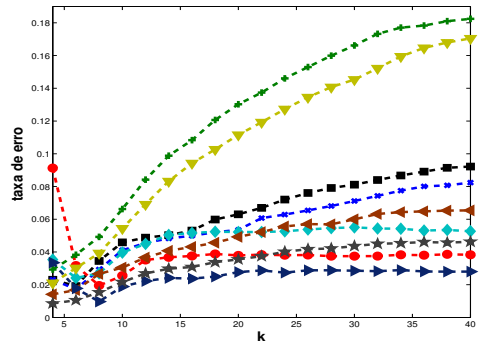

(e) RMGT

Figura 6.5: Avaliação dos métodos de geração de grafos na base de dados $\mathrm{COIL}_{2}$ usando as partições de 100 exemplos rotulados. 


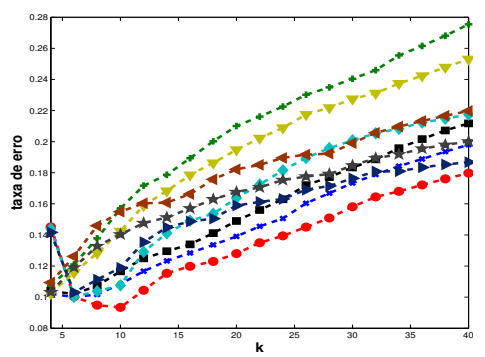

(a) GRF

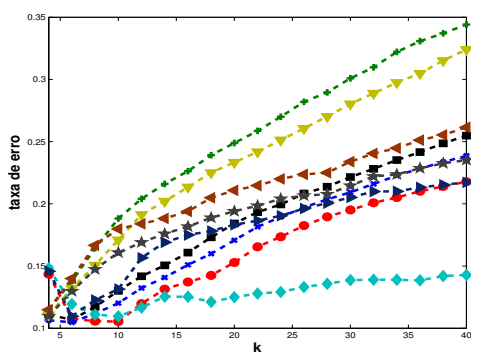

(b) LGC

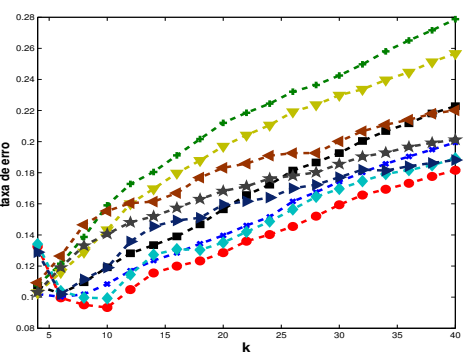

(c) LapRLS

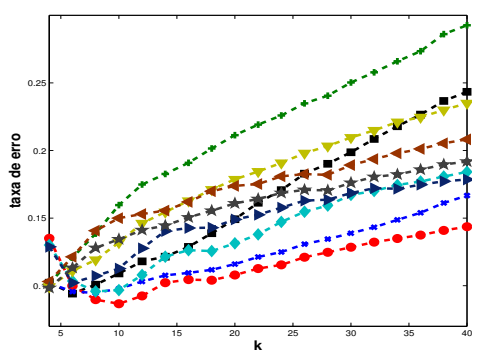

(d) LapSVM

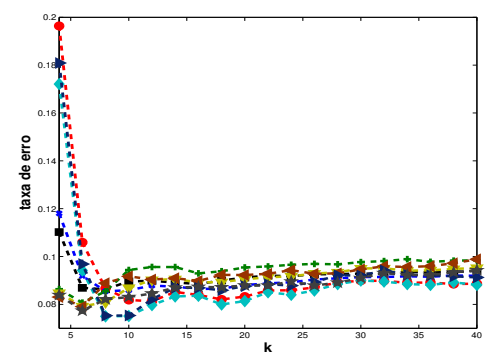

(e) RMGT

Figura 6.6: Avaliação dos métodos de geração de grafos na base de dados DIGIT-1 usando as partições de 10 exemplos rotulados.

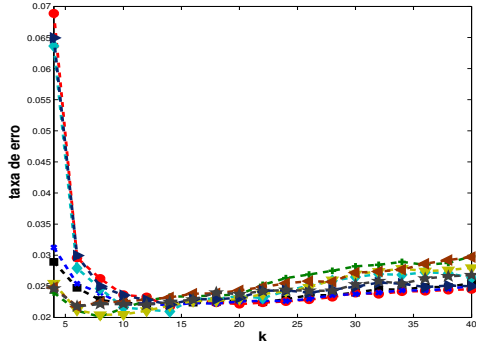

(a) GRF

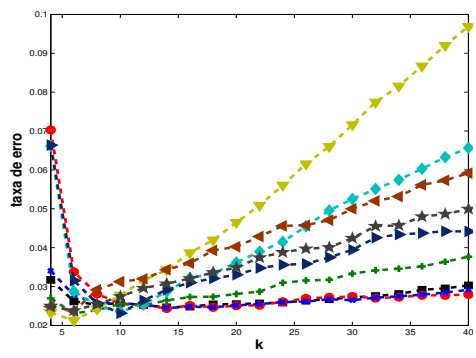

(b) LGC

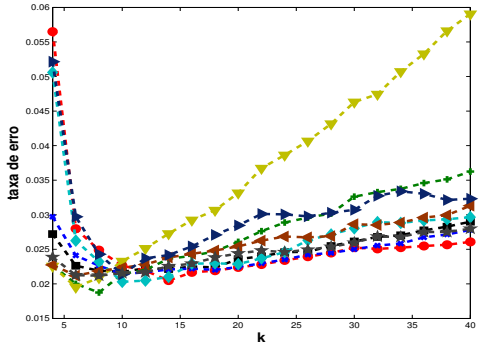

(c) LapRLS

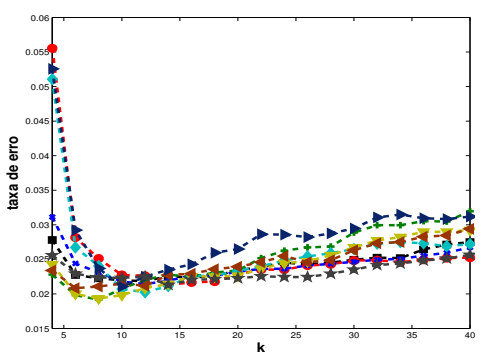

(d) LapSVM

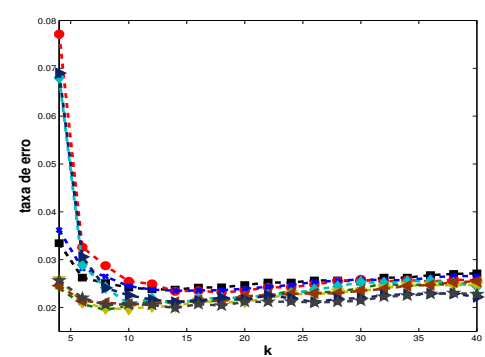

(e) RMGT

Figura 6.7: Avaliação dos métodos de geração de grafos na base de dados DIGIT-1 usando as partições de 100 exemplos rotulados. 


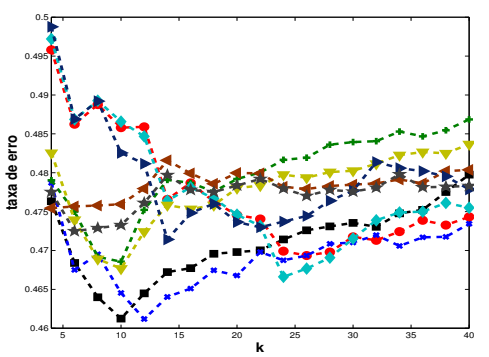

(a) GRF

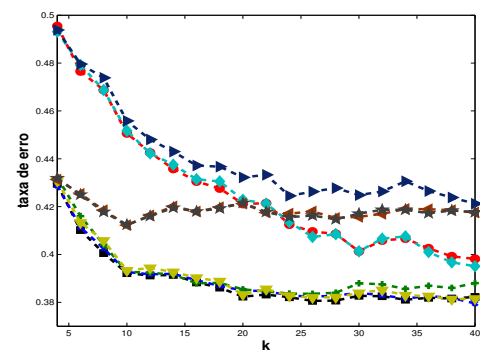

(b) LGC

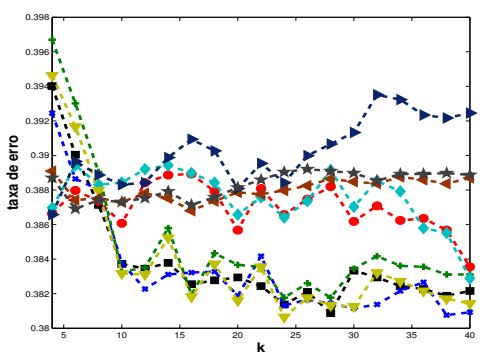

(c) LapRLS

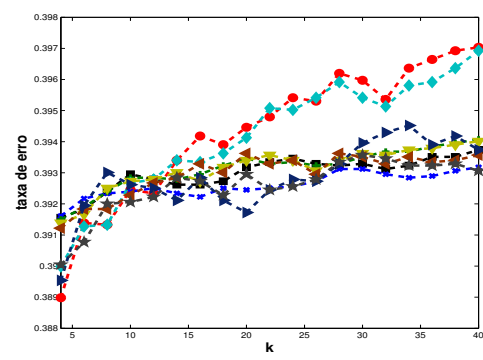

(d) LapSVM

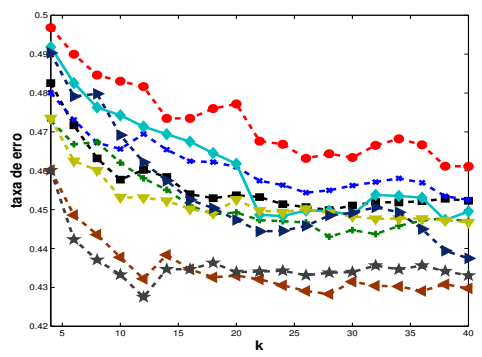

(e) RMGT

Figura 6.8: Avaliação dos métodos de geração de grafos na base de dados G-241N usando as partições de 10 exemplos rotulados.

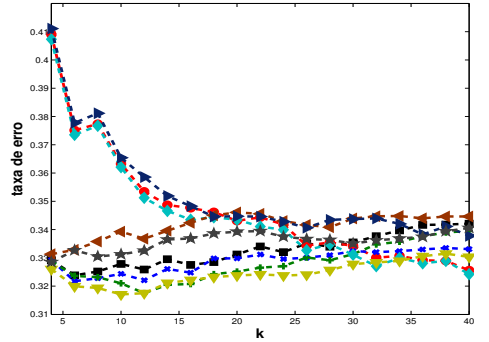

(a) GRF

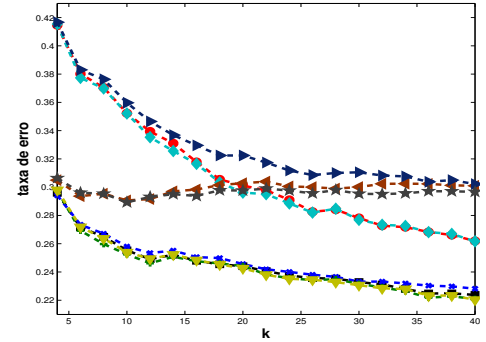

(b) LGC

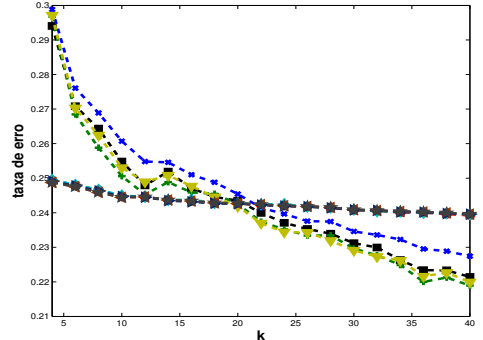

(c) LapRLS

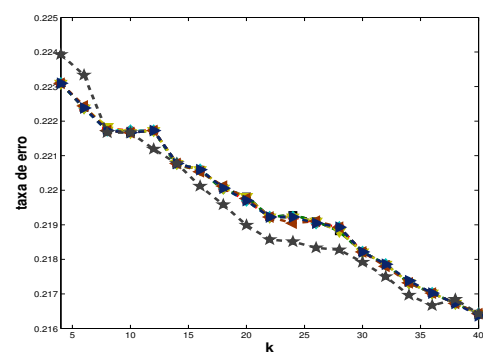

(d) LapSVM

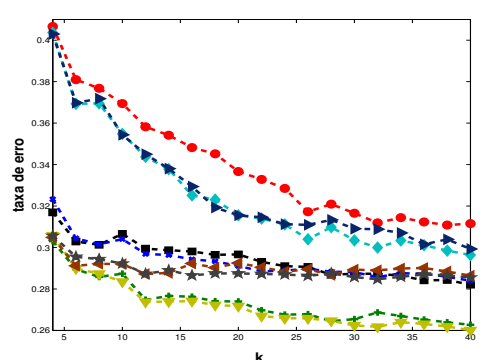

(e) RMGT

Figura 6.9: Avaliação dos métodos de geração de grafos na base de dados G-241N usando as partições de 100 exemplos rotulados. 


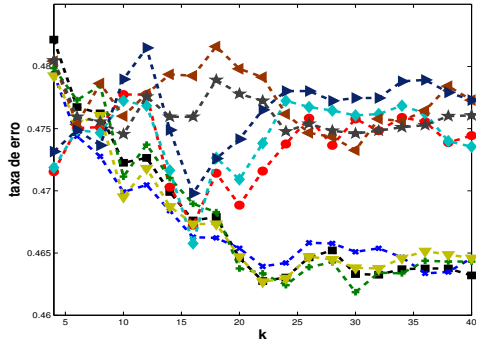

(a) GRF

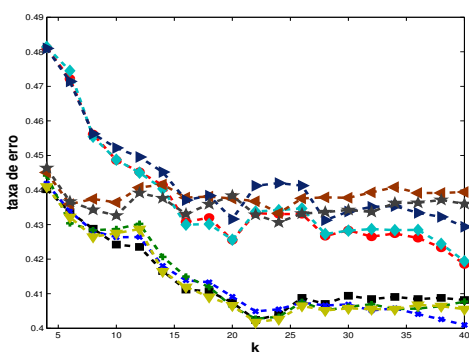

(b) LGC

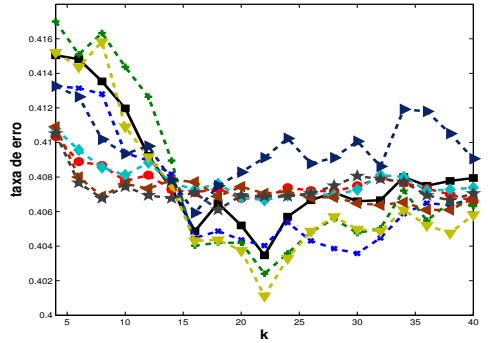

(c) LapRLS

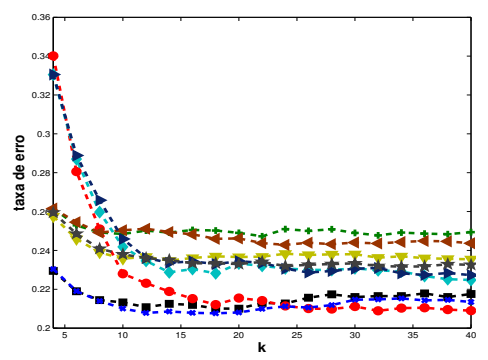

(d) LapSVM

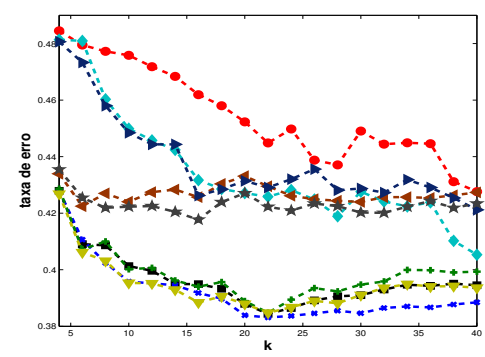

(e) RMGT

Figura 6.10: Avaliação dos métodos de geração de grafos nas bases de dados G-241C usando as partições de 10 exemplos rotulados.

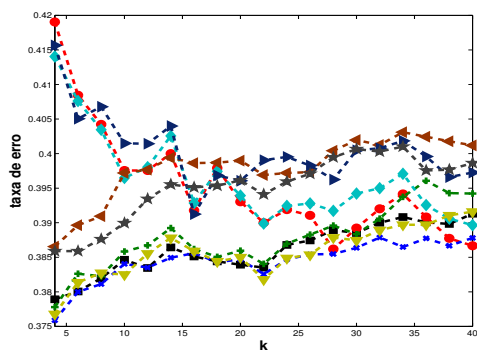

(a) GRF

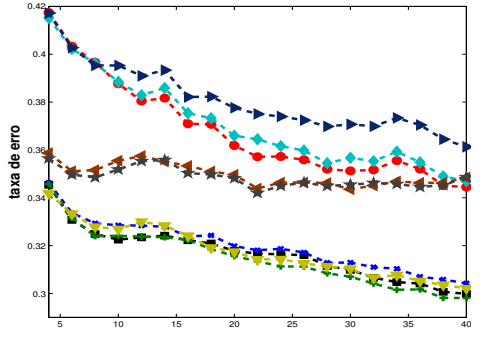

(b) LGC

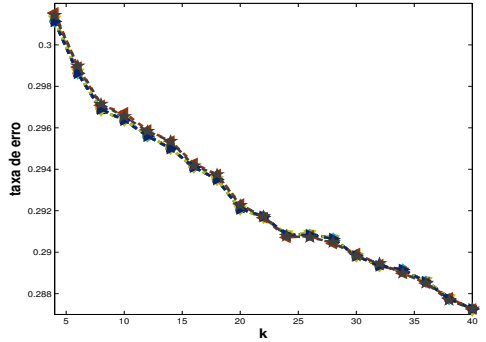

(c) LapRLS

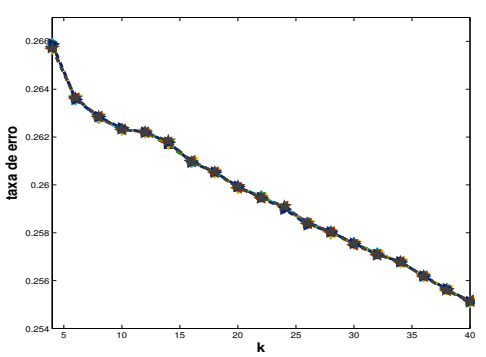

(d) LapSVM

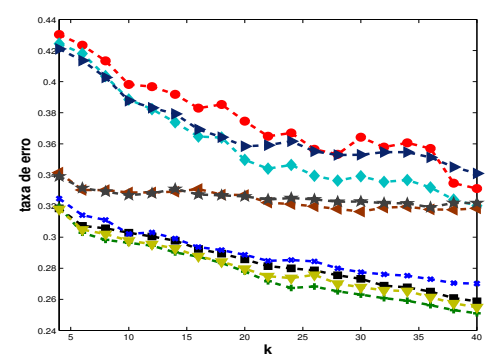

(e) RMGT

Figura 6.11: Avaliação dos métodos de geração de grafos na base de dados G-241C usando as partições de 100 exemplos rotulados. 


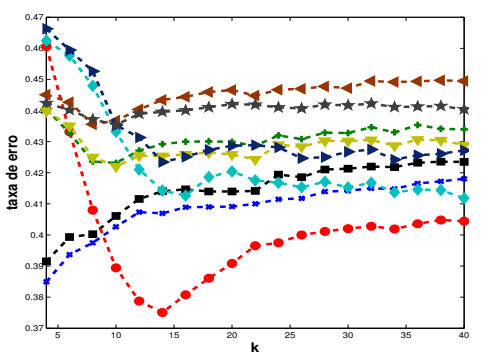

(a) GRF

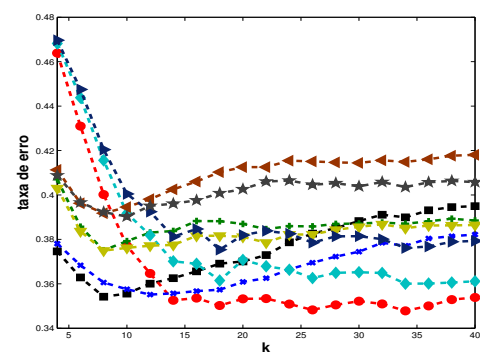

(b) LGC

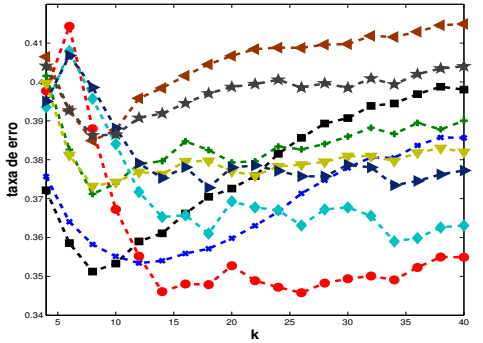

(c) LapRLS

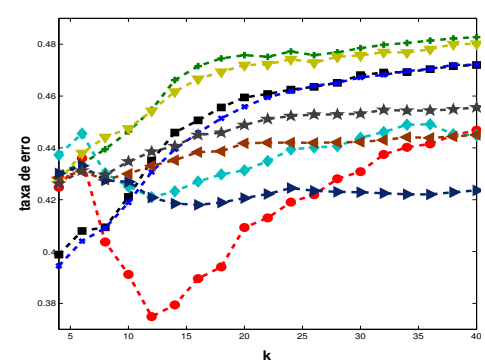

(d) LapSVM

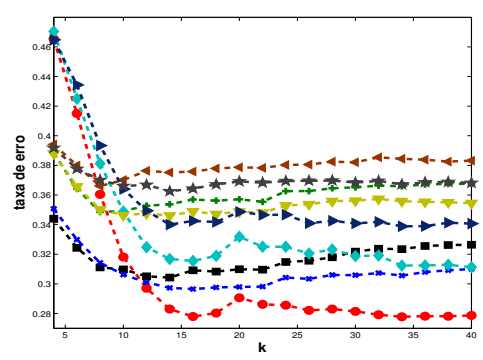

(e) RMGT

Figura 6.12: Avaliação dos métodos de geração de grafos na base de dados TEXT usando as partições de 10 exemplos rotulados.

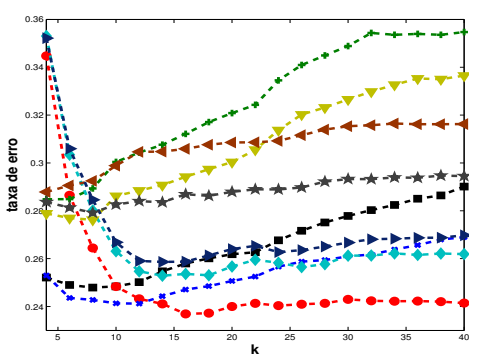

(a) GRF

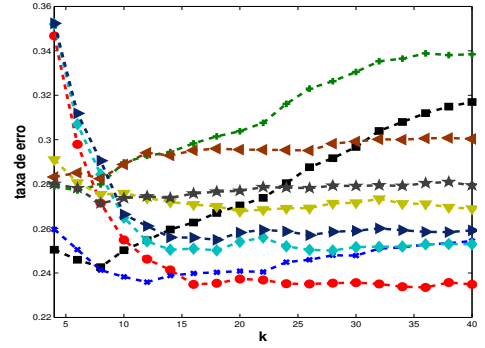

(b) LGC

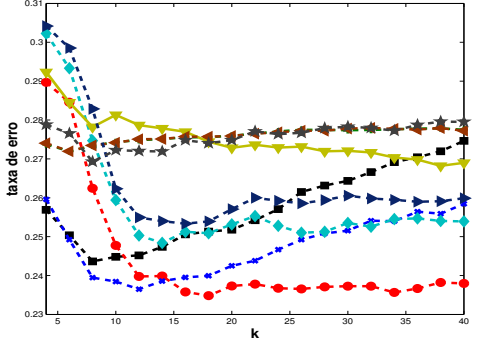

(c) LapRLS

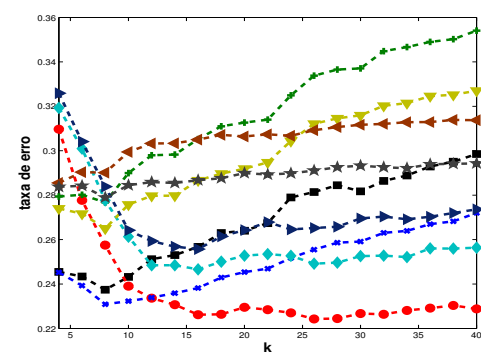

(d) LapSVM

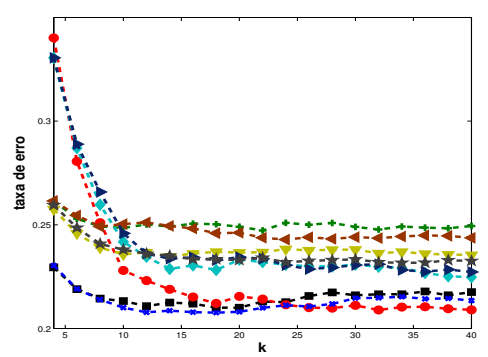

(e) RMGT

Figura 6.13: Avaliação dos métodos de geração de grafos na base de dados TEXT usando as partições de 100 exemplos rotulados. 


\subsection{Avaliação dos resultados obtidos}

A partir da análise dos resultados obtidos, pode-se extrair as seguintes constatações empíricas:

- Na Figura 6.2, nota-se uma elevada instabilidade dos grafos mutKNN$\mathrm{RBF}$, mutKNN-HM e mutKNN-LLE para valores relativamente pequenos de $k$. O grafo mutKNN-RBF obteve boa estabilidade para os algoritmos GRF, LGC, LapRLS e LapSVM quando $k \geq 10$. Entretanto, essa estabilidade não é evidenciada para o algoritmo RMGT. Os grafos symKNN-HM, symFKNN-HM, symKNN-LLE e symFKNN-LLE obtiveram as últimas posições no ranking dos grafos na maioria dos casos para todos os classificadores. Fixando-se o método de geração de matrizes ponderadas, nota-se que os grafos gerados a partir do grafo mutKNN apresentaram melhores desempenhos de classificação e estabilidade que os gerados a partir dos grafos symKNN e symFKNN para todos os classificadores, na maioria dos casos, para $k \geq 10$. O único caso em que a superioridade do grafo mutKNN em relação aos grafos symKNN e symFKNN não é evidenciada ocorre ao se usar o algoritmo LapRLS para $k \geq 30$. Para essa configuração, o grafo symFKNN-LLE obteve o melhor desempenho de classificação que o grafo mutKNN-LLE. Fixando-se o método de geração de grafos de adjacência, nota-se que os grafos gerados usando o kernel RBF apresentaram melhores desempenhos de classificação e estabilidade que os gerados usando as funções de similaridade HM e LLE para todos os classificadores. Ainda mais, nota-se que os grafos gerados a partir do kernel RBF apresentaram boa estabilidade para os algoritmos GRF, LGC e LapRLS quando $k \geq 10$;

- Na Figura 6.3, nota-se que os grafos symKNN-RBF, mutKNN-RBF, symFKNNRBF, mutKNN-HM e mutKNN-LLE obtiveram bons desempenho de classificação e estabilidade para os algoritmos GRF e LapRLS, enquanto que os demais grafos não obtiveram resultados satisfatórios para esses algoritmos. Fixando-se o método de geração de matrizes ponderadas, nota-se que os grafos gerados a partir do grafo mutKNN apresentaram melhores desempenho de classificação e estabilidade que os gerados a partir dos grafos symKNN e symFKNN para todos os classificadores, na maioria dos casos, para $k \geq 10$. O único caso em que a superioridade do grafo mutKNN em relação aos grafos symKNN e symFKNN não é evidenciada ocorre ao se usar o algoritmo LGC para $k \geq 30$. Para essa configuração, o grafo symFKNN-LLE obteve o melhor desempenho de classificação que o grafo mutKNN-LLE. Há uma elevada instabilidade dos grafos mutKNNRBF, mutKNN-HM e mutKNN-LLE para o algoritmo LGC quando $k \leq 10$. Os grafos symKNN-HM e symFKNN-HM apresentaram alta instabilidade para os algoritmos GRF, LapRLS e LapSVM;

- Na Figura 6.4, nota-se que há uma elevada variação nos rankings dos grafos, o que atrapalha demasiadamente o processo de análise experi- 
mental. Para exemplificar esse problema, toma-se como exemplo o comportamento do grafo symKNN-RBF para o algoritmo GRF. Nessa situação, para $k=8$, o grafo symKNN-RBF obteve a primeira posição no ranking dos grafos, enquanto que, para $k=40$, esse grafo obteve a sétima posição nesse ranking. Há relativamente poucas trocas de rankings entre os grafos ao se usar o algoritmo RMGT e todos os grafos apresentaram boa estabilidade para esse algoritmo quando $k \geq 8$. Entretanto, os grafos symKNN-RBF, mutKNN-RBF e symFKNN-RBF apresentaram alta instabilidade ao se usar o algoritmo RMGT quando $k \in\{4,6\}$. Os grafos symKNN-HM e symFKNN-HM obtiveram os piores desempenhos de classificação para os algoritmos GRF, LGC, LapRLS e LapSVM;

- Na Figura 6.5, percebe-se que os grafos symKNN-HM e symFKNN-HM não obtiveram resultados satisfatórios para nenhum dos algoritmos. Os demais grafos obtiveram comportamentos similares para todos os algoritmos. Entretanto, nota-se uma pequena instabilidade do grafo mutKNNRBF ao se usar o algoritmo RMGT com $k=4$, enquanto que os demais grafos apresentaram resultados estáveis;

- Na Figura 6.6, nota-se que os grafos mutKNN-RBF, mutKNN-HM e mutKNN-LLE apresentaram elevada instabilidade ao se usar o algoritmo RMGT com $k=4$. Na maioria dos casos, todos os grafos apresentaram comportamentos similares para todos os algoritmos quando $k \geq 6$. Nota-se que o grafo mutKNN-HM é o único grafo que apresentou boa estabilidade para o algoritmo LGC. Há uma elevada variância no ranking dos grafos. Por exemplo, o grafo mutKNN-HM obteve a última posição no ranking dos grafos para o algoritmo LGC quando $k=4$. Entretanto, para $k \geq 14$, o grafo mutKNN-HM obteve a primeira posição nesse ranking para o mesmo algoritmo;

- Na Figura 6.7, nota-se que os grafos mutKNN-RBF, mutKNN-HM e mutKNN-LLE apresentaram elevada instabilidade para todos os algoritmos quando $k=4$. Nota-se ainda uma elevada instabilidade do grafo symFKNNHM para os algoritmos LGC e LapRLS para $k \geq 16$. Entretanto, esse grafo obteve a primeira posição no ranking dos grafos para os algoritmos LGC e LapRLS quando $k=6$. O grafo mutKNN-HM obteve a primeira posição no ranking dos grafos para os algoritmos LGC e LapRLS quando $k=6$. Entretanto, para esses algoritmos e para $k \geq 16$, o grafo mutKNN-HM obteve a última posição nesse ranking, não apresentando desempenho de classificação competitivo com os demais grafos;

- Na Figura 6.8, nota-se que há uma elevada alternância no ranking dos grafos para todos os algoritmos. Os grafos mutKNN-RBF, mutKNN-HM e mutKNN-LLE apresentaram alta instabilidade para o algoritmo LGC. Entretanto, o desempenho de classificação do algoritmo LGC usando esses grafos aumenta conforme a conectividade do grafo aumenta. Todos os grafos apresentaram comportamentos similares para os algoritmos LapRLS e LapSVM com pequenas variações nas taxas de erro. Observa-se 
uma elevada variância nos rankings dos grafos para os algoritmos GRF e LapRLS. Por exemplo, para $k \in\{8,10\}$, o grafo symKNN-RBF obteve a primeira posição no ranking dos grafos para o algoritmo GRF, enquanto que, para $k=40$, esse grafo obteve a sexta posição nesse ranking. Para $k=4$, o grafo symFKNN-HM obteve a oitava posição no ranking dos grafos para o algoritmo LapRLS, enquanto que, para $k=40$, esse grafo obteve a segunda posição nesse ranking;

- Na Figura 6.9, nota-se que os grafos mutKNN-RBF, mutKNN-HM, symKNNLLE, mutKNN-LLE e symFKNN-LLE obtiveram alta estabilidade para o algoritmo LapRLS, enquanto que os demais grafos foram instáveis para esse algoritmo. Todos os grafos apresentaram comportamentos similares para o algoritmo LapSVM. Os grafos mutKNN-RBF, mutKNN-HM e mutKNN-LLE apresentaram resultados instáveis para os algoritmos GRF, LGC e RMGT, enquanto que os demais grafos apresentaram boa estabilidade para esse algoritmo. Observa-se uma elevada variância nos rankings dos grafos para o algoritmo LapRLS. Por exemplo, para $k=4$, o grafo symFKNN-HM obteve a oitava posição no ranking dos grafos para o algoritmo LapRLS, enquanto que, para $22 \leq k \leq 32$, esse grafo obteve a primeira posição nesse ranking;

- Na Figura 6.10, nota-se que os grafos symKNN-RBF, symFKNN-RBF, symKNN-HM e symFKNN-HM obtiveram os melhores desempenhos de classificação para os algoritmos GRF, LGC e RMGT. Os grafos mutKNNRBF, mutKNN-HM e mutKNN-LLE apresentaram alta instabilidade para o algoritmo LapSVM quando $k \leq 8$, enquanto que os demais grafos apresentaram resultados estáveis para esse algoritmo para todos os valores de $k$. Apesar de os grafos symKNN-LLE e symFKNN-LLE apresentarem boa estabilidade para os algoritmos LGC e RMGT, os resultados referentes a esses grafos não são competitivos com os resultados dos grafos symKNN-RBF, symFKNN-RBF, symKNN-HM e symFKNN-HM para esses algoritmos. Observa-se uma alta variância no ranking dos grafos para os algoritmos GRF, LGC, LapRLS e RMGT. Por exemplo, para $k=4$, o grafo symKNN-RBF obteve a última posição no ranking dos grafos para o algoritmo GRF, enquanto que, para $k=40$, esse grafo obteve a primeira posição nesse ranking;

- Na Figura 6.11, nota-se que todos os grafos apresentaram comportamentos similares para os algoritmos LapRLS e LapSVM. Os grafos symKNNRBF, symFKNN-RBF, symKNN-HM e symFKNN-HM apresentaram bons desempenho de classificação e estabilidade para os algoritmos LGC e RMGT. Entretanto, esses grafos não apresentaram desempenhos de classificação competitivos com os grafos symKNN-RBF, symFKNN-RBF, symKNN-HM e symFKNN-HM para os algoritmos LGC e RMGT para valores relativamente grandes de $k$. Para o algoritmo GRF, nota-se uma elevada variância nos rankings dos grafos. Observa-se uma alta variância no ranking dos grafos para o algoritmo GRF. Por exemplo, para $k=4$, o 
grafo mutKNN-RBF obteve a última posição no ranking dos grafos para o algoritmo GRF, enquanto que, para $k=40$, esse grafo obteve a primeira posição nesse ranking;

- Na Figura 6.12, nota-se que todos os grafos obtiveram boa estabilidade para os algoritmos GRF, LGC e RMGT quando $k \geq 21$. Os grafos mutKNNRBF, mutKNN-HM e mutKNN-LLE apresentaram alta instabilidade para os algoritmos GRF, LGC, LapRLS e RMGT para valores relativamente pequenos de $k$. Há uma elevada variância nos rankings dos grafos, o que dá margem à elaboração de conclusões enviesadas. Para exemplificar esse problema, toma-se como exemplo o comportamento dos grafos symKNNRBF e mutKNN-RBF para o algoritmo LapRLS. Nessa situação, para $k=6$, o grafo symKNN-RBF obteve o melhor desempenho de classificação, enquanto que o grafo mutKNN-RBF ficou na última posição do ranking dos grafos. Entretanto, para $k \geq 26$, o grafo mutKNN-RBF obteve a primeira posição no ranking dos grafos, enquanto que o grafo symKNN-RBF obteve a sétima posição nesse ranking;

- Na Figura 6.13, nota-se que os grafos mutKNN-RBF, mutKNN-HM e mutKNN-LLE apresentaram elevada instabilidade para valores relativamente pequenos de $k$ em todos os algoritmos. Para os algoritmos GRF, LGC, LapRLS e LapSVM, há uma alta variância no ranking dos grafos. Essa alta variância é evidenciada no comportamento do grafo symKNN-RBF, o qual obteve as duas primeiras posições no ranking dos grafos para valores pequenos de $k$ e as últimas posições nesse ranking para valores grandes de $k$. Para $k=4$, o grafo mutKNN-RBF obteve a última posição no ranking dos grafos para o algoritmo RMGT, enquanto que, para $k \geq 28$, esse grafo obteve a primeira posição nesse ranking. Situações parecidas com essa ocorrem para os demais algoritmos.

\subsection{Considerações finais}

Neste capítulo, propôs-se um modelo de avaliação experimental para avaliar a estabilidade dos métodos de geração de grafos para cada base de dados e cada classificador. A partir da análise dos resultados, constatou-se que há uma alta variância no ranking dos grafos, o que dificulta tirar conclusões gerais e dá margem à elaboração de conclusões tendenciosas. Vários resultados diferentes do esperado foram discutidos ao longo deste capítulo.

Acredita-se que o modelo de avaliação experimental proposto neste capítulo deva ser amplamente usado na literatura de aprendizado semissupervisionado. Com o uso desse modelo de avaliação experimental, a quantidade de conclusões tendenciosas referentes à avaliação da estabilidade dos métodos de geração de grafos seria drasticamente reduzida, o que aumentaria a maturidade empírica da área de aprendizado semissupervisionado. Como o modelo de avaliação experimental proposto neste capítulo não leva em conta a informação referente aos exemplos rotulados, ele poderia ser usado, por exemplo, 
para avaliar a estabilidade de métodos de geração de grafos na área de agrupamento espectral. Acredita-se que o uso do modelo proposto neste capítulo possa melhorar a qualidade dos trabalhos empíricos na área de aprendizado de máquina. 



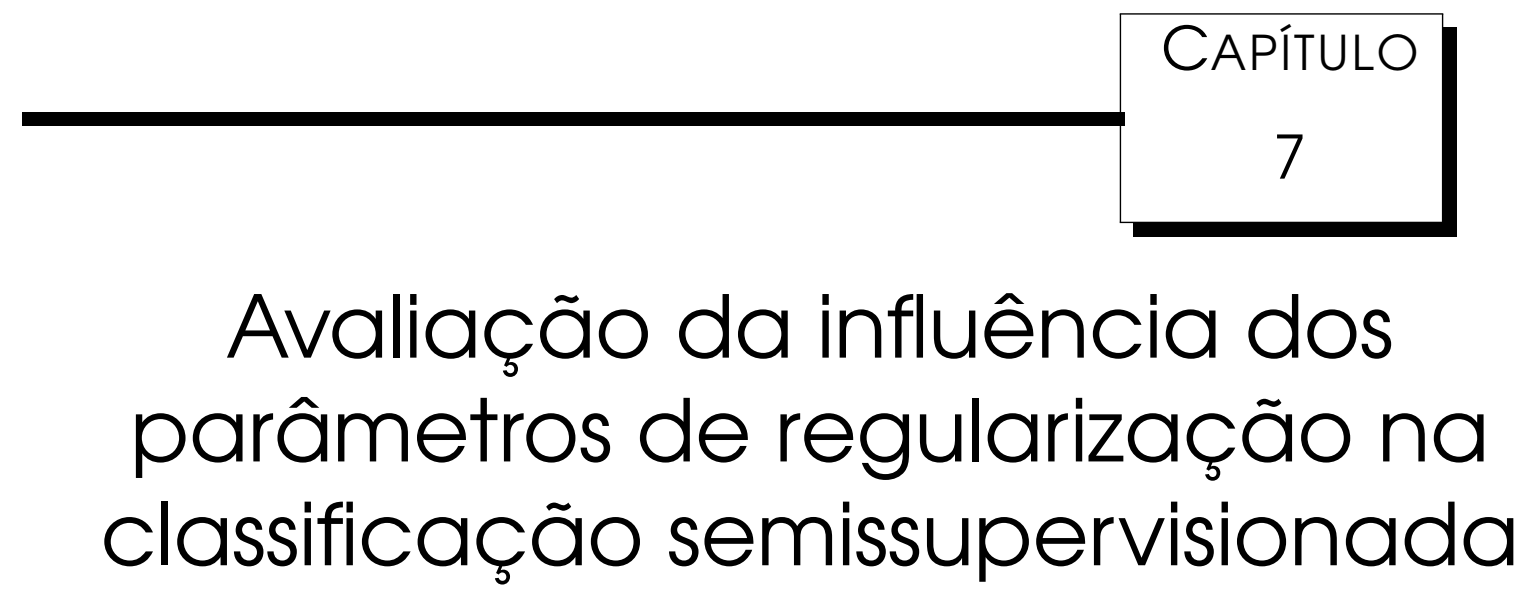

Neste capítulo, propõe-se um modelo de avaliação experimental que visa avaliar a influência que os parâmetros de regularização $\gamma_{A}$ e $\gamma_{I}$ apresentam no desempenho de classificação dos algoritmos LapRLS e LapSVM. Em outras palavras, procura-se avaliar como os parâmetros de regularização influenciam a taxa de erro dos algoritmos LapRLS e LapSVM dado um método de geração de grafos e uma base pré-fixados. Portanto, o modelo de avaliação experimental proposto neste capítulo é uma ferramenta inestimável para verificar quais classificadores apresentam um bom balanço entre desempenho de classificação e estabilidade com respeito a um dado conjunto de valores dos parâmetros de regularização.

A avaliação da influência do parâmetro de regularização $\mu$ no desempenho de classificação do algoritmo LGC também poderia ser feita neste capítulo. Entretanto, para evitar uma explosão combinatória de resultados, optou-se por remover essa avaliação deste trabalho. Os resultados dessa avaliação apontaram que os valores 0.01 e 0.05 sempre geraram os melhores resultados em relação aos demais valores e as curvas de erro do algoritmo LGC em relação à $\mu$ tiveram comportamentos bastante similares.

Este capítulo está organizado como a seguir. Na Seção 7.1, descreve-se o modelo de avaliação da influência dos parâmetros de regularização na classificação semissupervisionada, proposto neste capítulo. Na Seção 7.2, discutemse os resultados obtidos tendo como foco a análise das superfícies de erro geradas pelos algoritmos LapRLS e LapSVM em relação aos parâmetros de regularização. Por fim, na Seção 7.3, faz-se as considerações finais deste capítulo. 


\subsection{Descrição do modelo experimental proposto}

O intuito do modelo experimental proposto neste capítulo é analisar como que a taxa de erro dos algoritmos LapRLS e LapSVM é influenciada pelos valores dos parâmetros de regularização $\gamma_{A}$ e $\gamma_{I}$. Em outras palavras, pretende-se avaliar a estabilidade dos classificadores semissupervisionados com respeito ao valor dos parâmetros de regularização. Como há dois parâmetros a serem analisados, os resultados mostrados neste capítulo são superfícies de erro, ao invés de curvas de erro. Entretanto, a ideia de avaliação de estabilidade segue a mesma linha de raciocínio apresentada nos Capítulos 5 e 6.

A avaliação da influência dos parâmetros de regularização é feita fixandose cada base de dados e cada método de geração de grafos. Com isso, pode-se avaliar a superfície de erro gerada pelos classificadores semissupervisionados, variando-se os valores dos parâmetros de regularização. Essa avaliação de estabilidade visa comparar a estabilidade dos algoritmos LapRLS e LapSVM usando uma variedade de métodos de geração de grafos. Com isso, as superfícies de erro geradas a partir de um dado grafo são comparadas com as demais.

Como é inviável analisar todas as combinações de grafos com todas as combinações de parâmetros de regularização, fixa-se o valor de $k$ do grafo $k \mathrm{NN}$ que obteve o melhor resultado na análise de melhor caso e então variamse os valores dos parâmetros de regularização $\gamma_{A}$ e $\gamma_{I}$, conforme descrito na Seção 3.3. Para todos os classificadores, fixam-se os valores do parâmetro de esparsificação que obtiveram o melhor resultado na análise de melhor caso e então variam-se os valores dos parâmetros de regularização $\gamma_{A}$ e $\gamma_{I}$.

Todos os resultados apresentados neste capítulo seguem o padrão mostrado na Figura 7.1. Os resultados são gerados a partir do uso do protocolo experimental descrito no Capítulo 3. Os resultados obtidos para essa avaliação são mostrados nas Figuras 7.2 até 7.25 e discutidos na Seção 7.2.

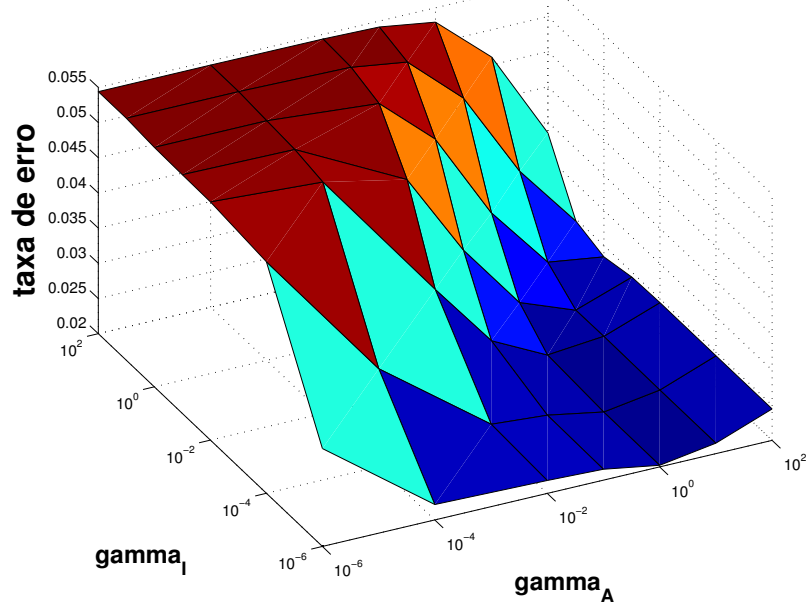

Figura 7.1: Padrão de gráfico para a avaliação da influência dos parâmetros de regularização na classificação semissupervisionada. 


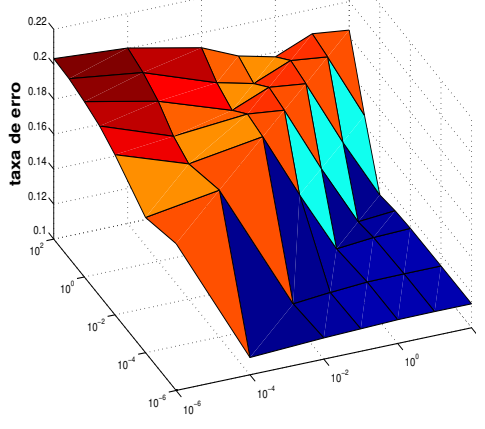

(a) symKNN-RBF

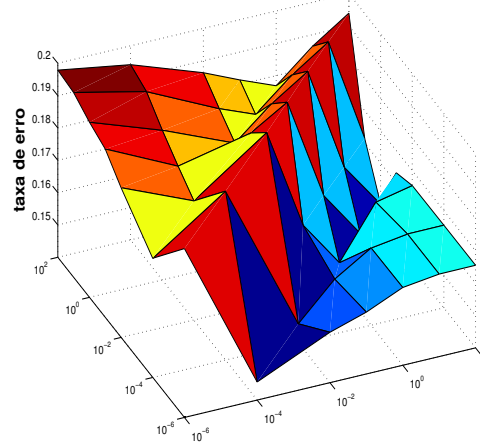

(d) symKNN-HM

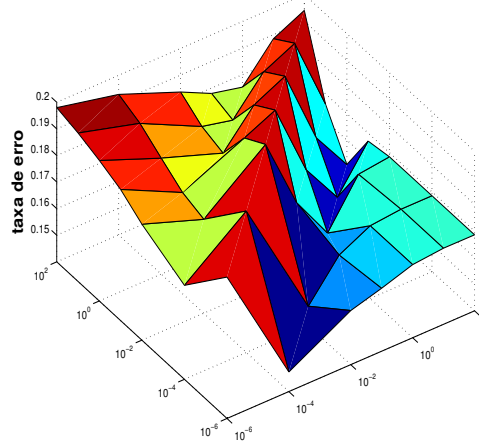

(g) symKNN-LLE

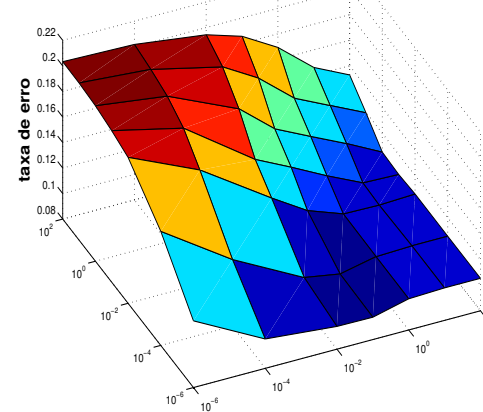

(b) mutKNN-RBF

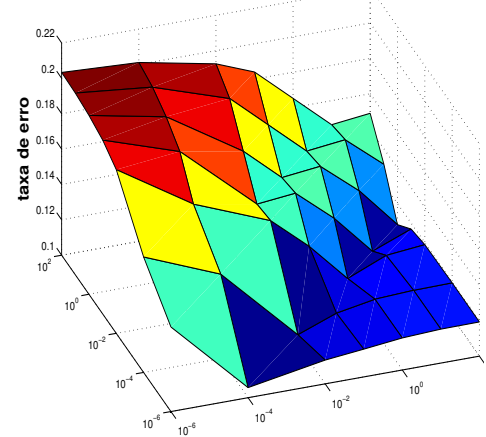

(e) mutKNN-HM

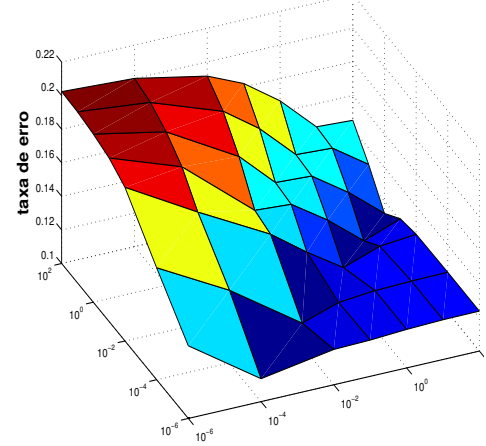

(h) mutKNN-LLE

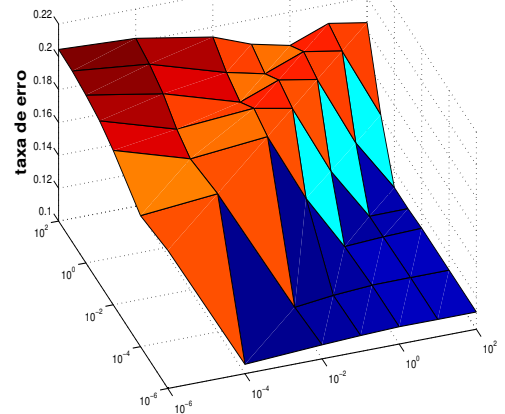

(c) symFKNN-RBF

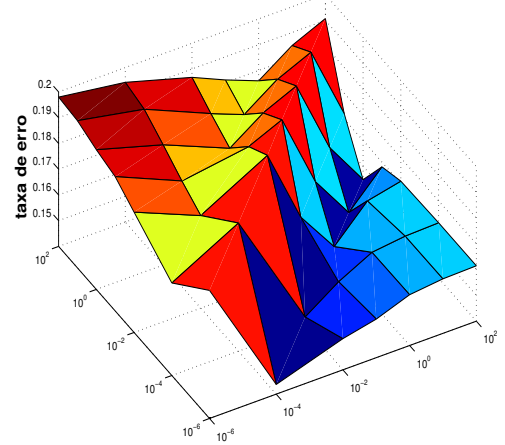

(f) symFKNN-HM

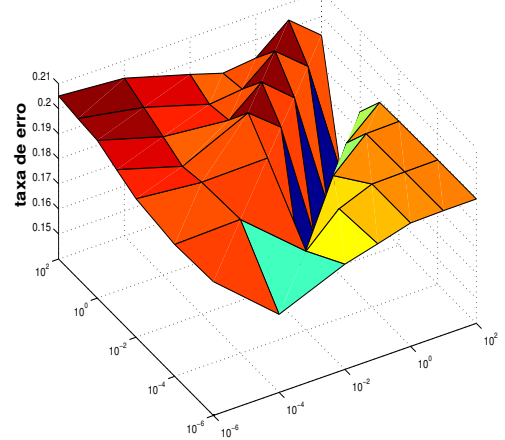

(i) symFKNN-LLE

Figura 7.2: Superfícies de erro do algoritmo LapRLS na base de dados USPS para as partições de 10 exemplos rotulados. 


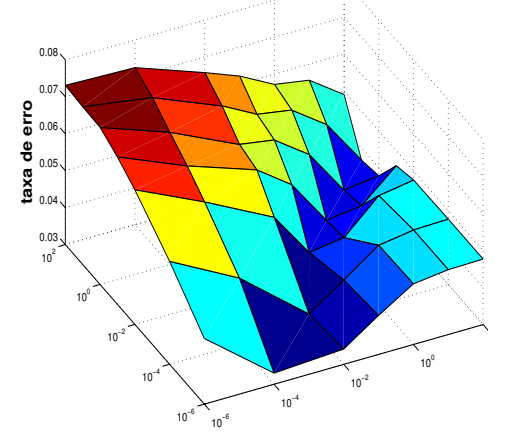

(a) symKNN-RBF

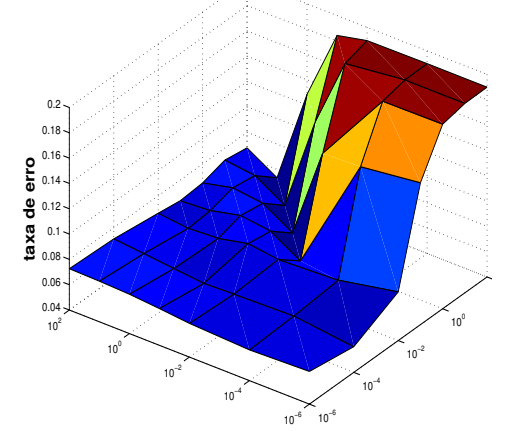

(d) symKNN-HM

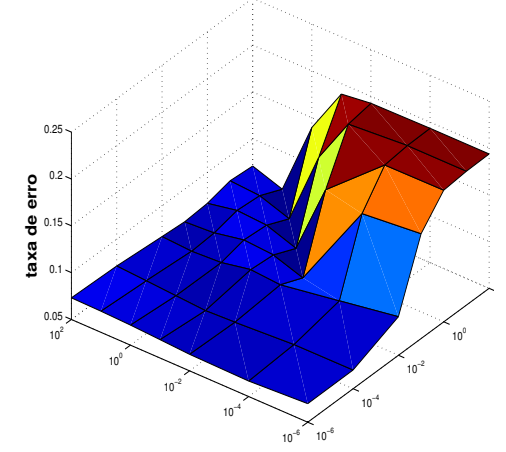

(g) symKNN-LLE

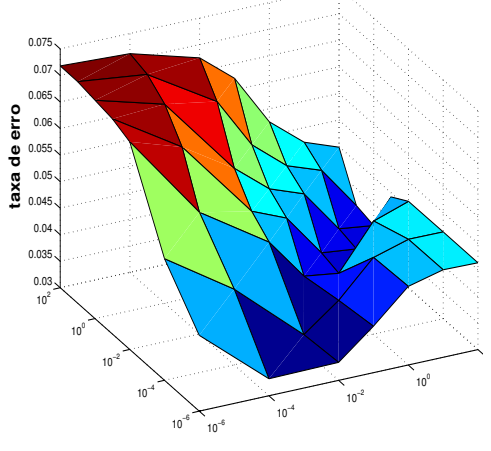

(b) mutKNN-RBF

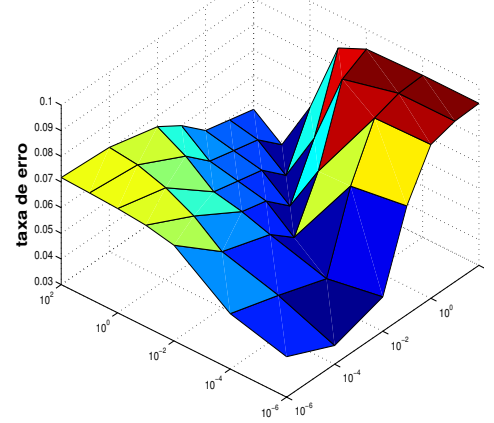

(e) mutKNN-HM

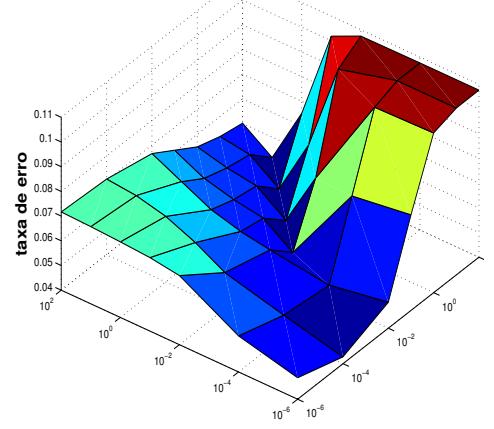

(h) mutKNN-LLE

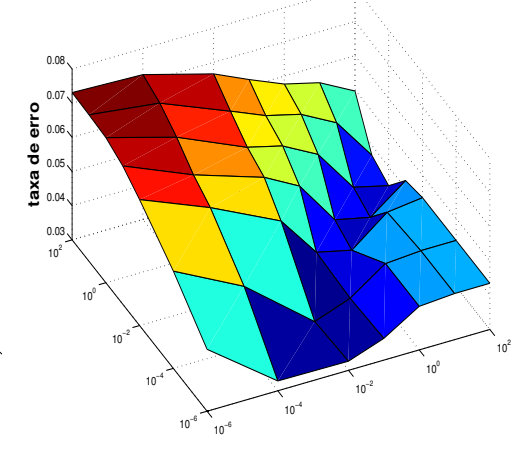

(c) symFKNN-RBF

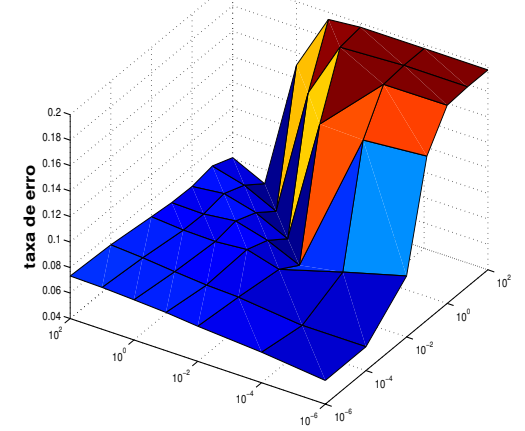

(f) symFKNN-HM

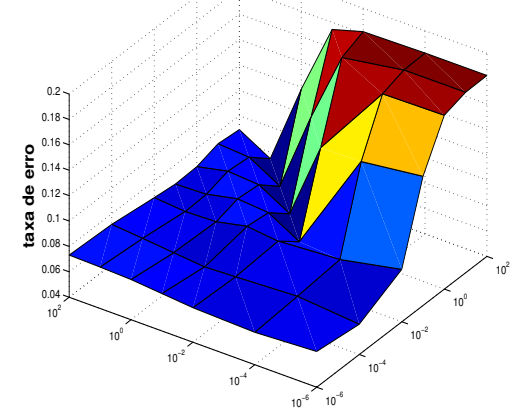

(i) symFKNN-LLE

Figura 7.3: Superfícies de erro do algoritmo LapRLS na base de dados USPS para as partições de 100 exemplos rotulados. 


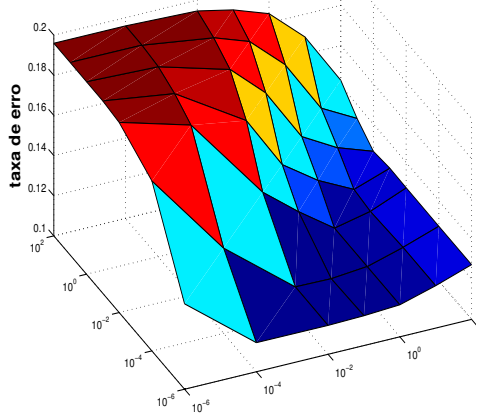

(a) symKNN-RBF

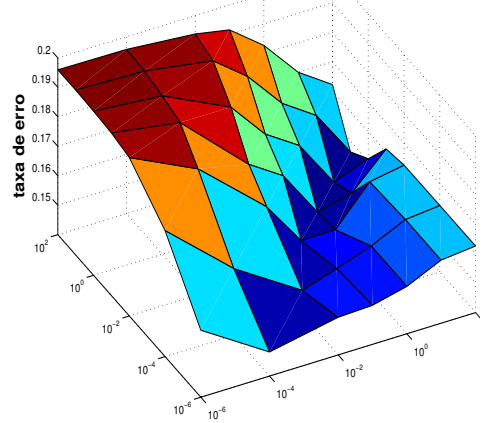

(d) symKNN-HM

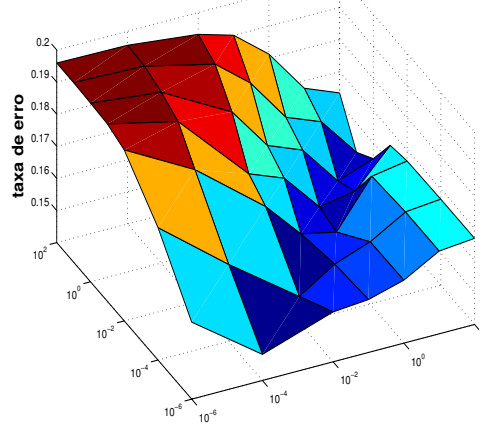

(g) symKNN-LLE

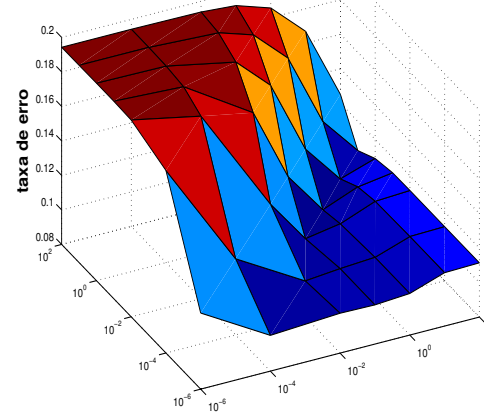

(b) mutKNN-RBF

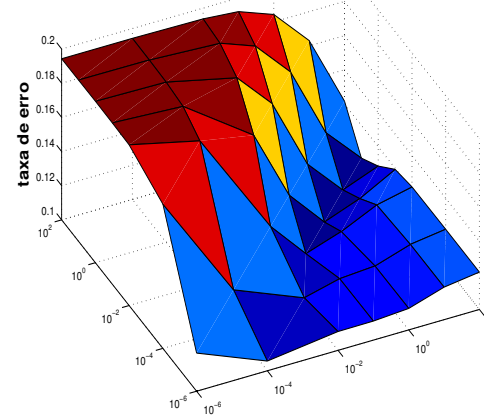

(e) mutKNN-HM

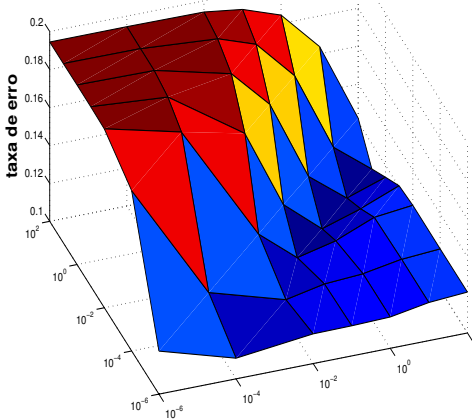

(h) mutKNN-LLE

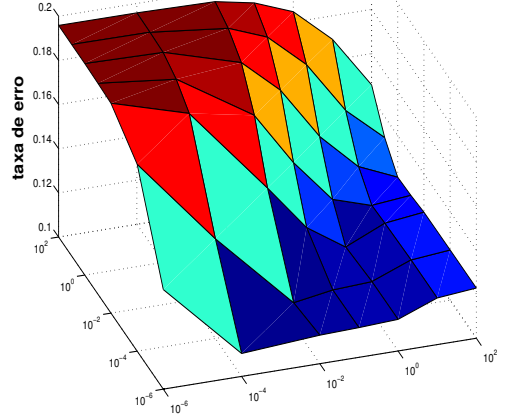

(c) symFKNN-RBF

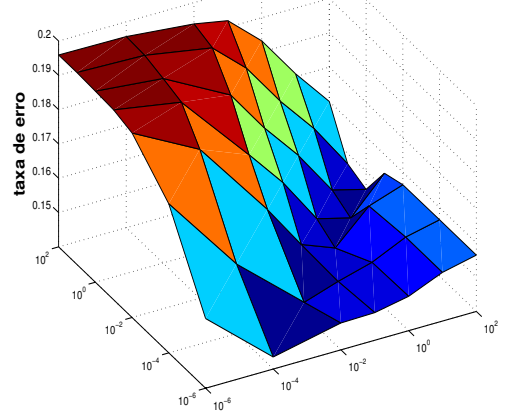

(f) symFKNN-HM

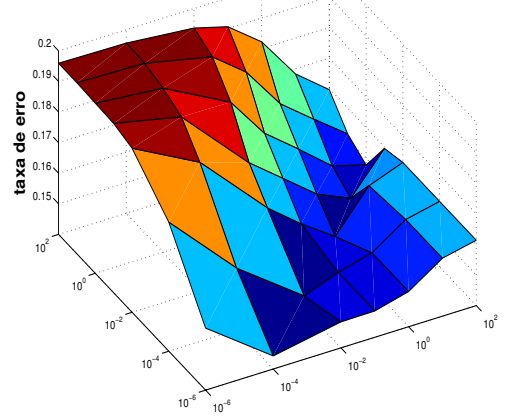

(i) SymFKNN-LLE

Figura 7.4: Superfícies de erro do algoritmo LapSVM na base de dados USPS para as partições de 10 exemplos rotulados. 


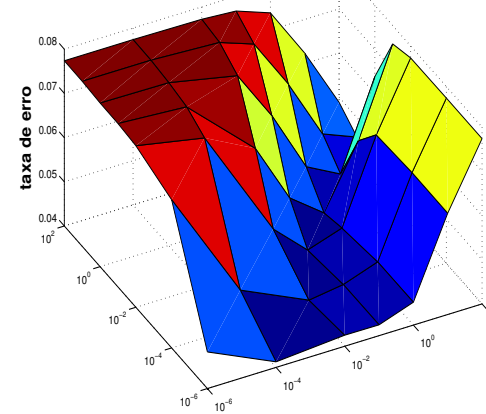

(a) symKNN-RBF

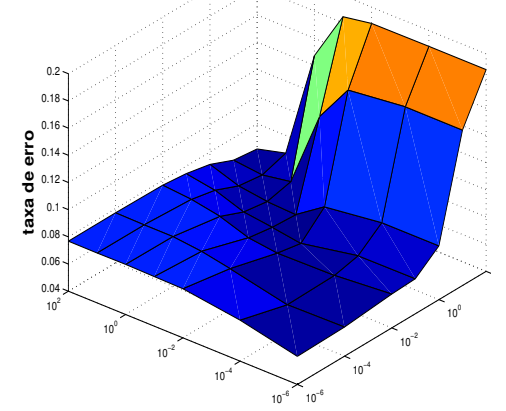

(d) symKNN-HM

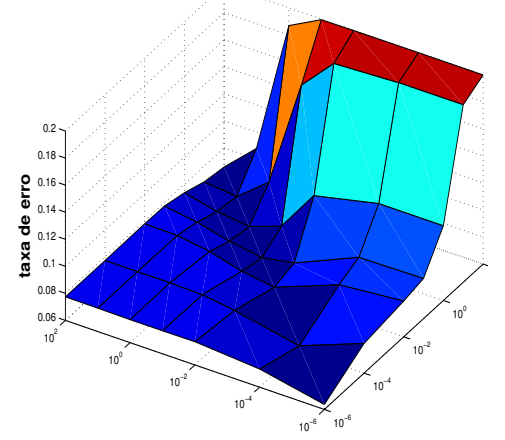

(g) symKNN-LLE

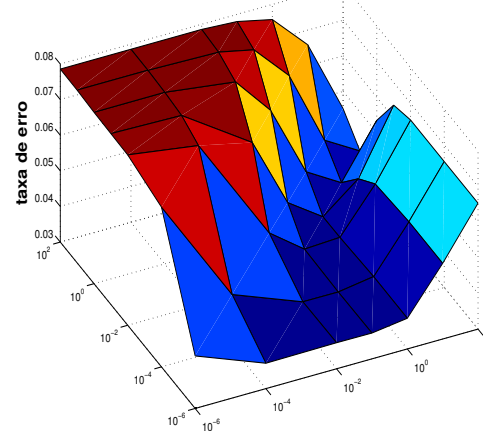

(b) mutKNN-RBF

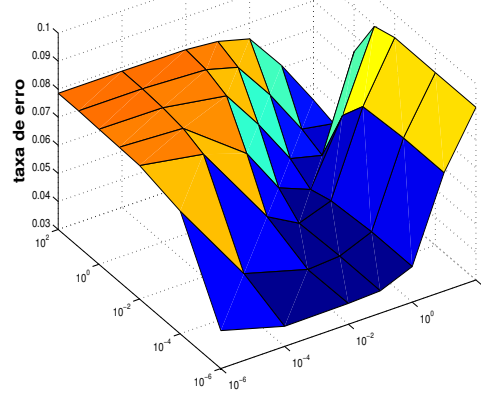

(e) mutKNN-HM

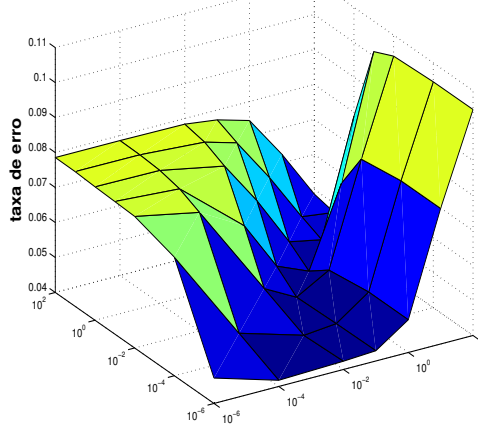

(h) mutKNN-LLE

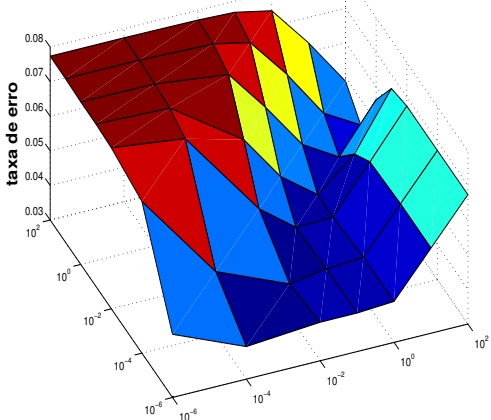

(c) symFKNN-RBF

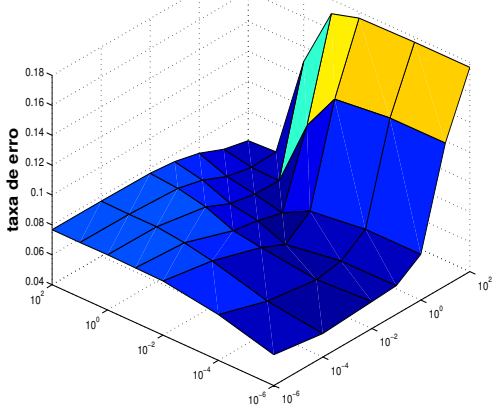

(f) symFKNN-HM

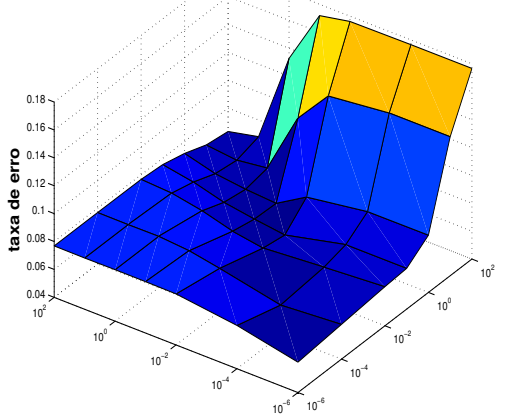

(i) SymFKNN-LLE

Figura 7.5: Superfícies de erro do algoritmo LapSVM na base de dados USPS para as partições de 100 exemplos rotulados. 


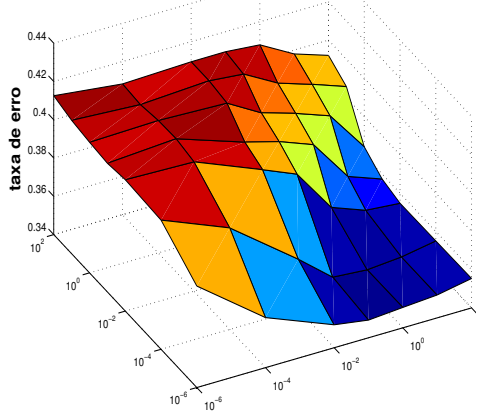

(a) symKNN-RBF

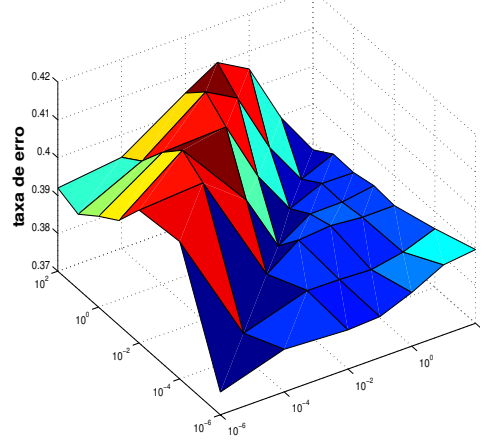

(d) symKNN-HM

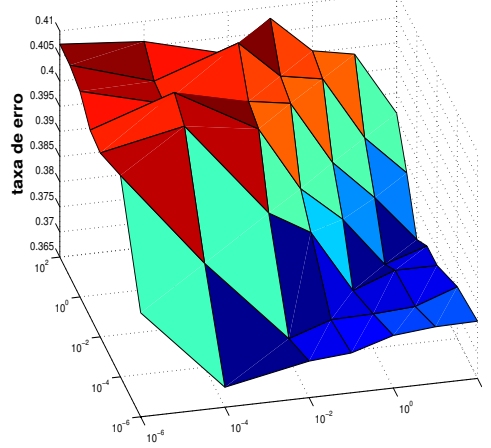

(g) symKNN-LLE

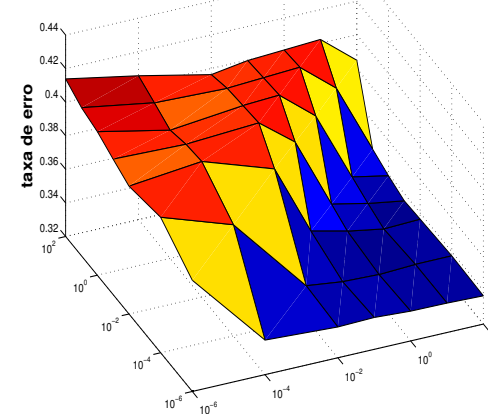

(b) mutKNN-RBF

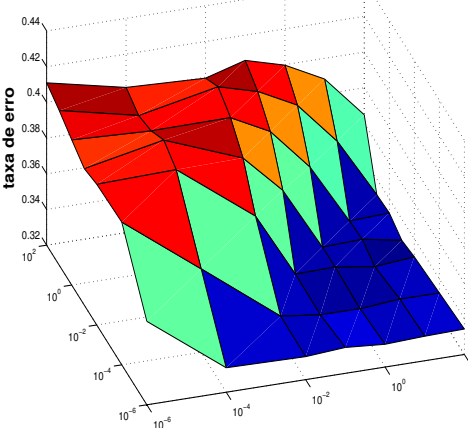

(e) mutKNN-HM

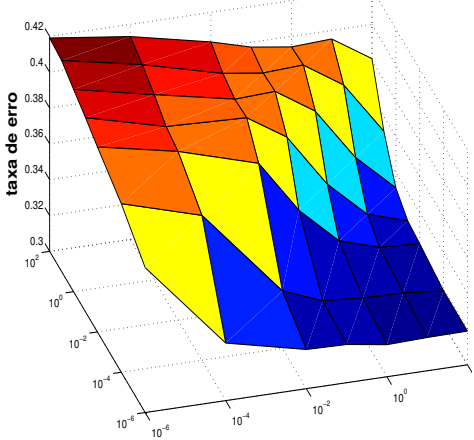

(h) mutKNN-LLE

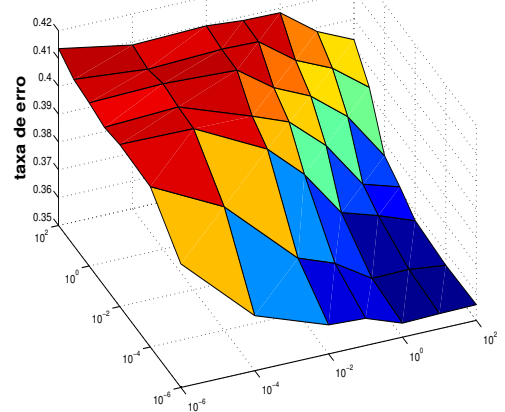

(c) symFKNN-RBF

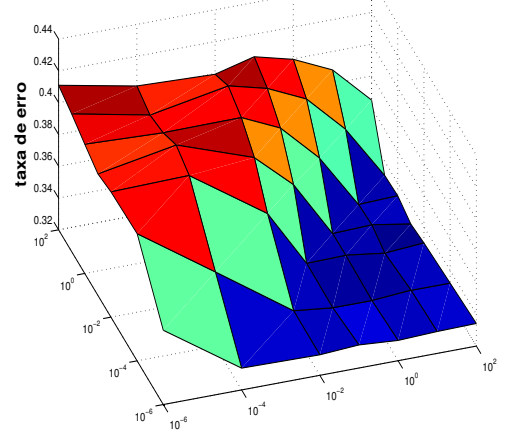

(f) symFKNN-HM

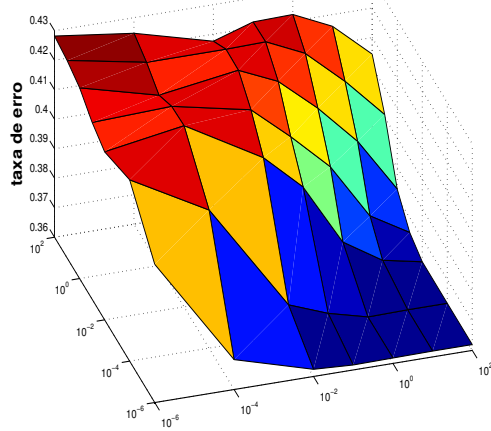

(i) symFKNN-LLE

Figura 7.6: Superfícies de erro do algoritmo LapRLS na base de dados $\mathrm{COIL}_{2}$ para as partições de 10 exemplos rotulados. 


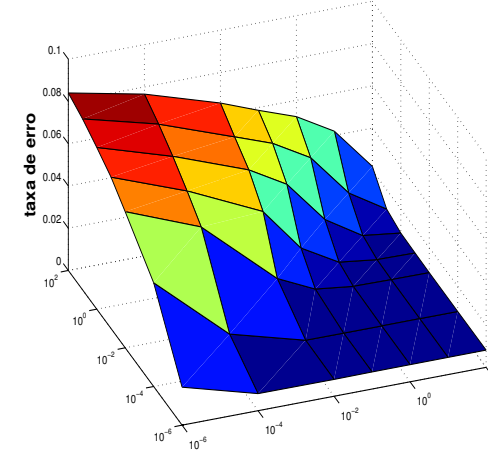

(a) symKNN-RBF

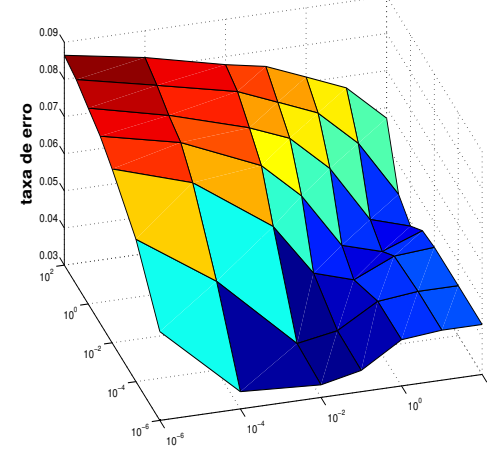

(d) symKNN-HM

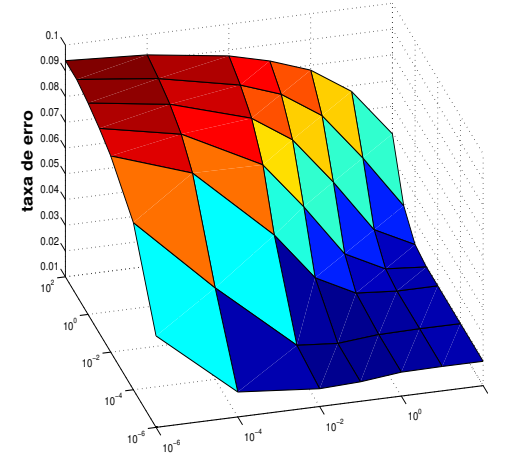

(g) symKNN-LLE

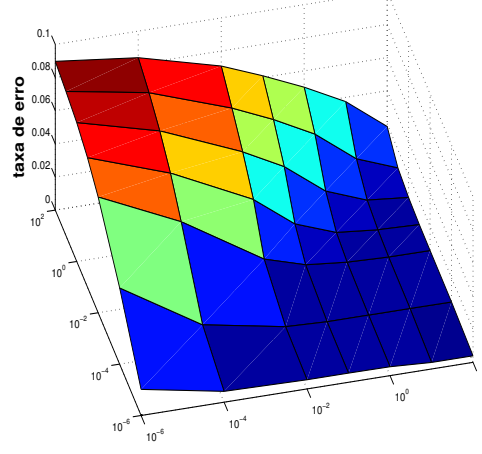

(b) mutKNN-RBF

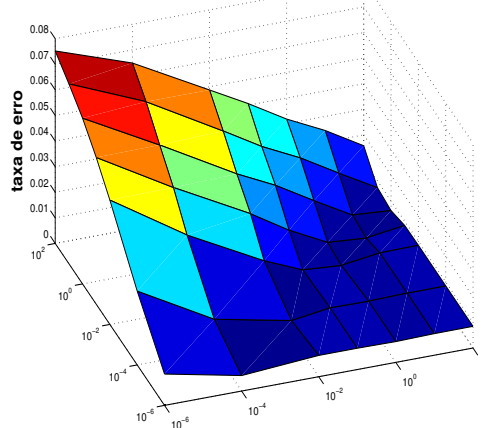

(e) mutKNN-HM

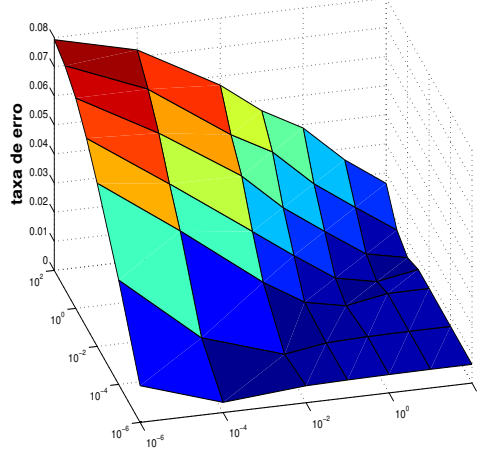

(h) mutKNN-LLE

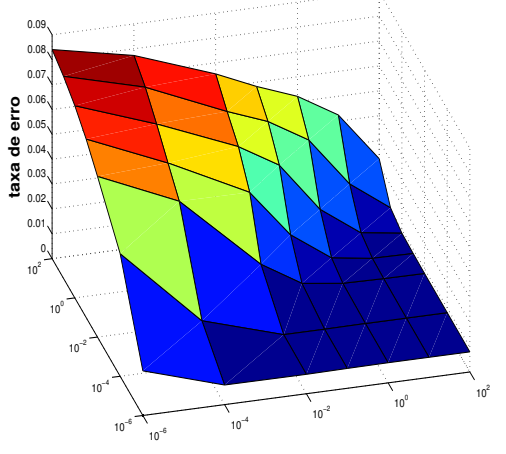

(c) symFKNN-RBF

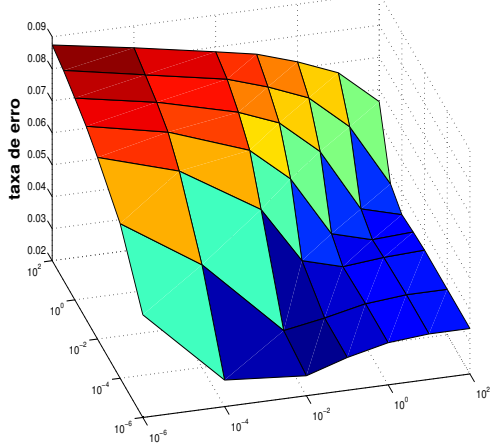

(f) symFKNN-HM

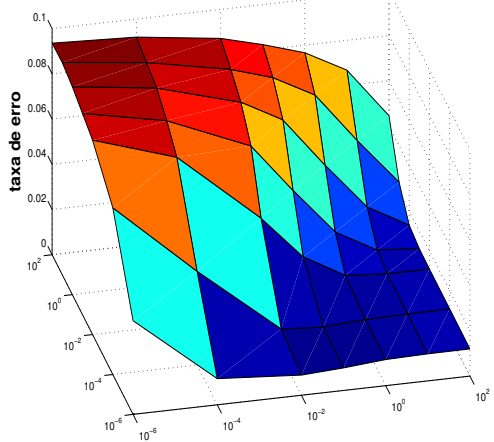

(i) SymFKNN-LLE

Figura 7.7: Superfícies de erro do algoritmo LapRLS na base de dados $\mathrm{COIL}_{2}$ para as partições de 100 exemplos rotulados. 


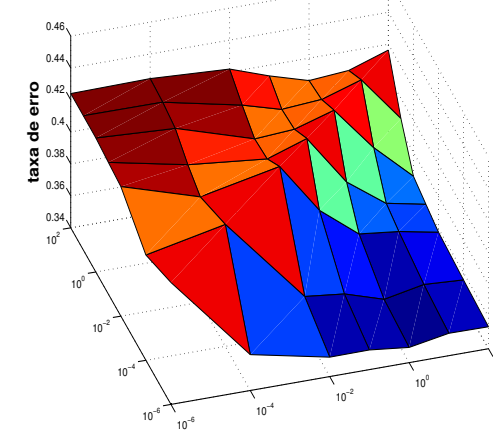

(a) symKNN-RBF

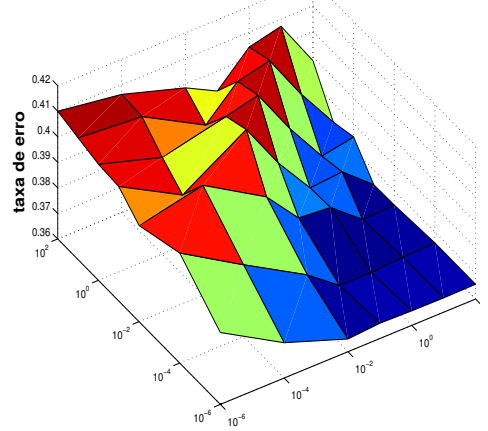

(d) symKNN-HM

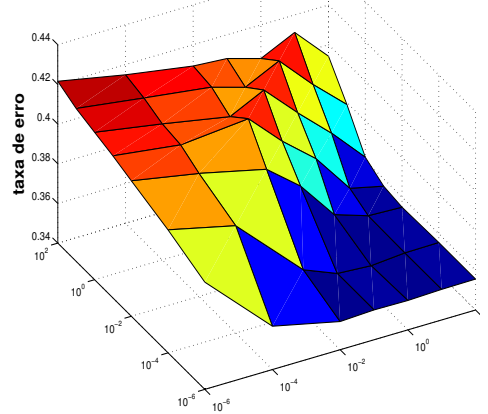

(g) symKNN-LLE

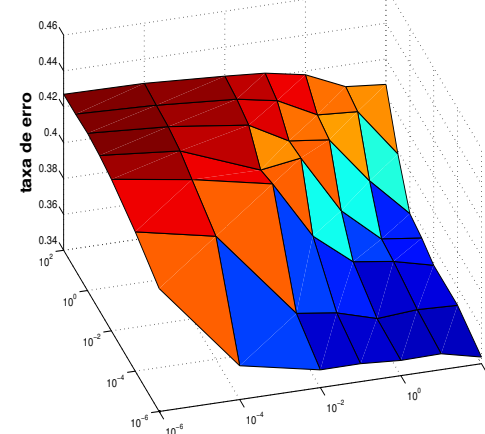

(b) mutKNN-RBF

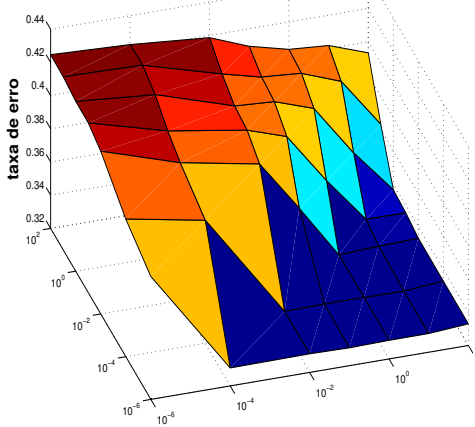

(e) mutKNN-HM

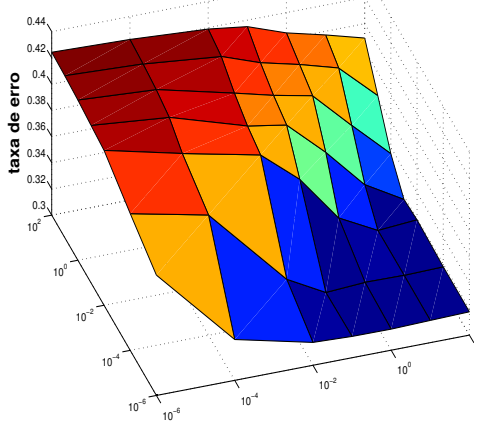

(h) mutKNN-LLE

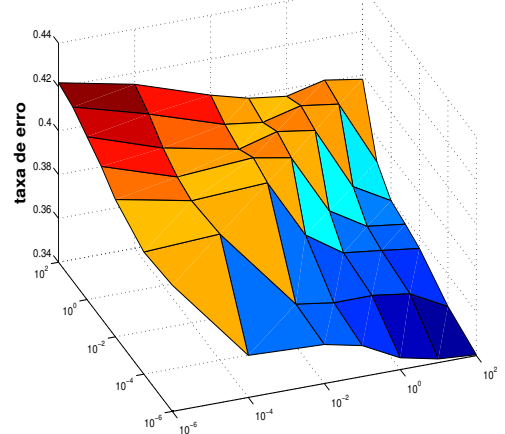

(c) symFKNN-RBF

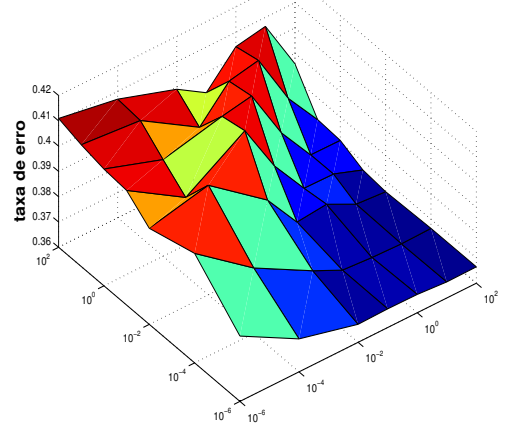

(f) symFKNN-HM

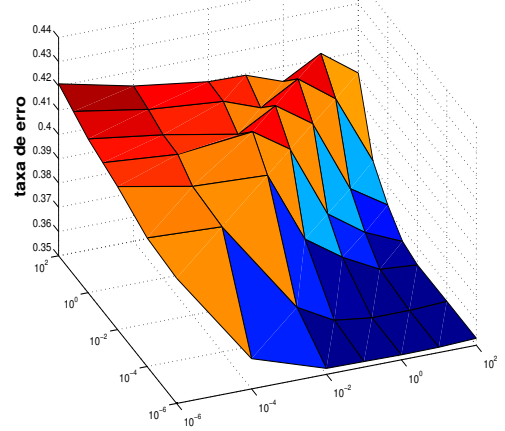

(i) SymFKNN-LLE

Figura 7.8: Superfícies de erro do algoritmo LapSVM na base de dados $\mathrm{COIL}_{2}$ para as partições de 10 exemplos rotulados. 


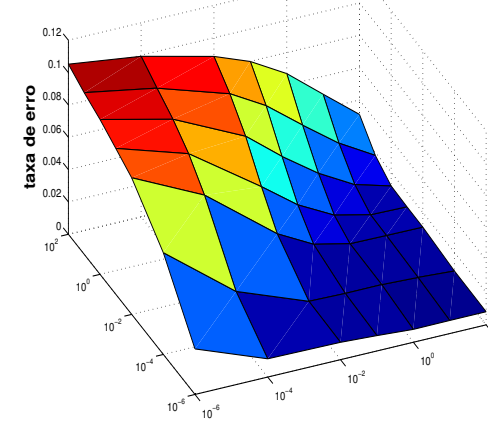

(a) symKNN-RBF

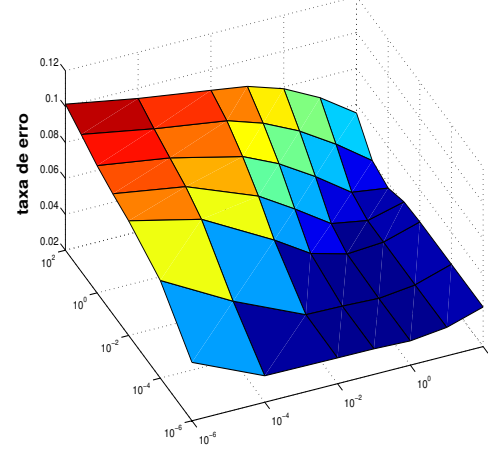

(d) symKNN-HM

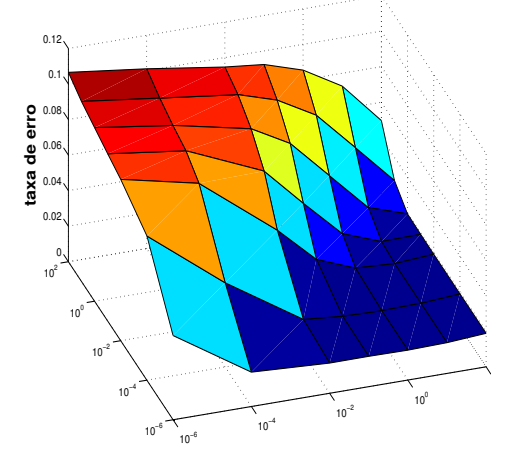

(g) symKNN-LLE

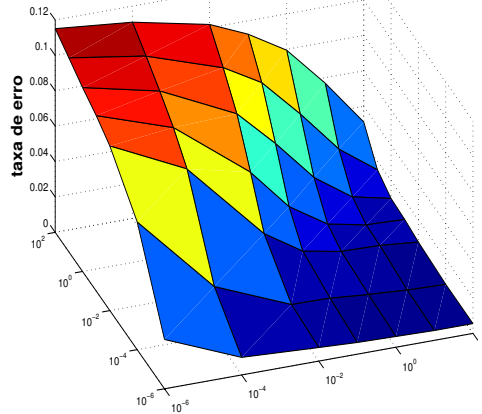

(b) mutKNN-RBF

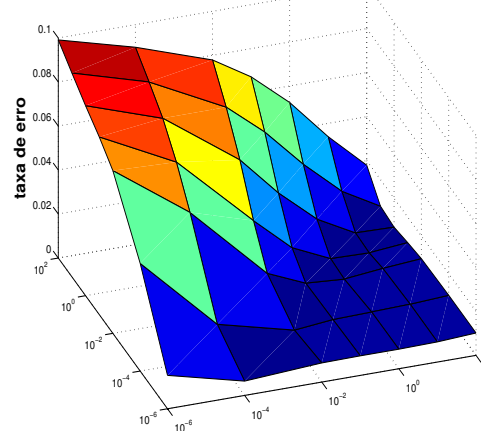

(e) mutKNN-HM

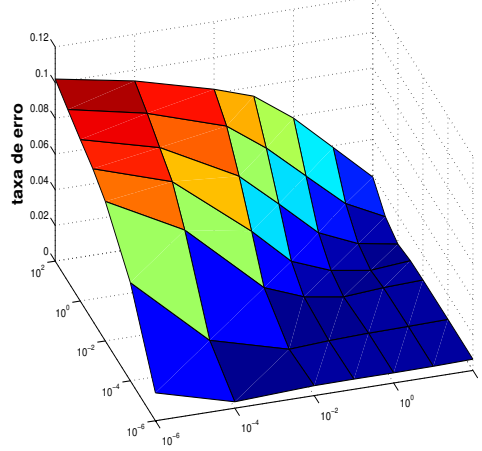

(h) mutKNN-LLE

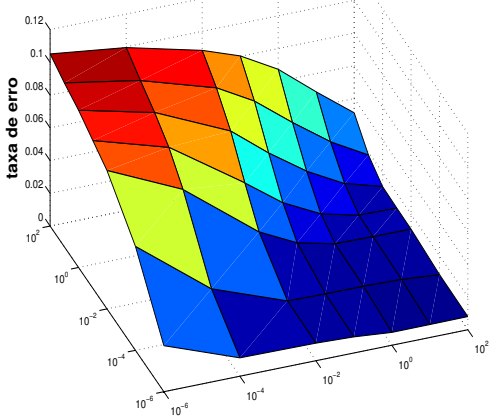

(c) symFKNN-RBF

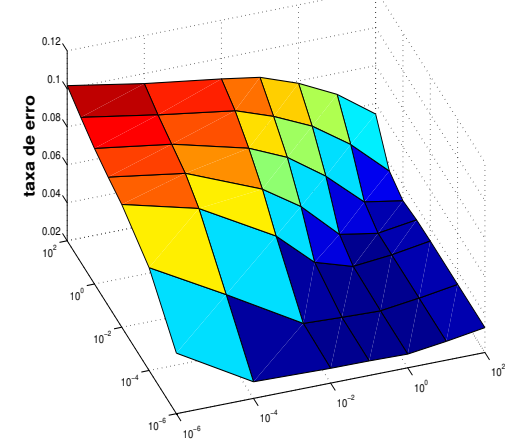

(f) symFKNN-HM

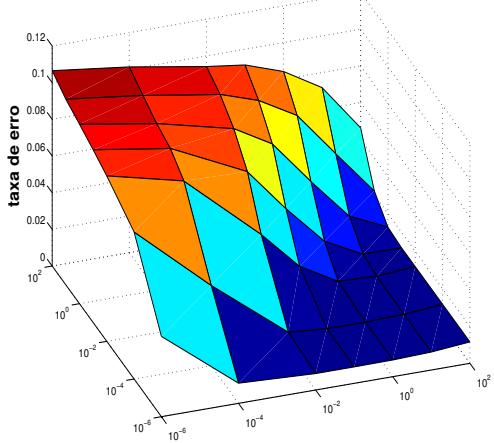

(i) SymFKNN-LLE

Figura 7.9: Superfícies de erro do algoritmo LapSVM na base de dados $\mathrm{COIL}_{2}$ para as partições de 100 exemplos rotulados. 


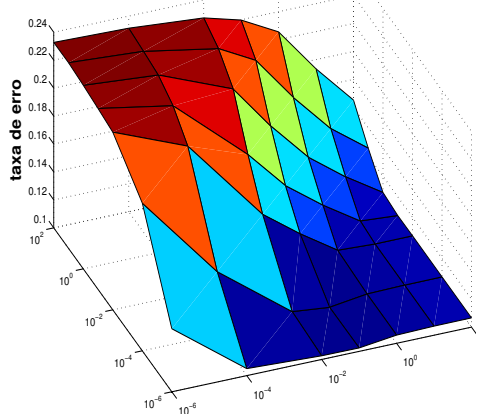

(a) symKNN-RBF

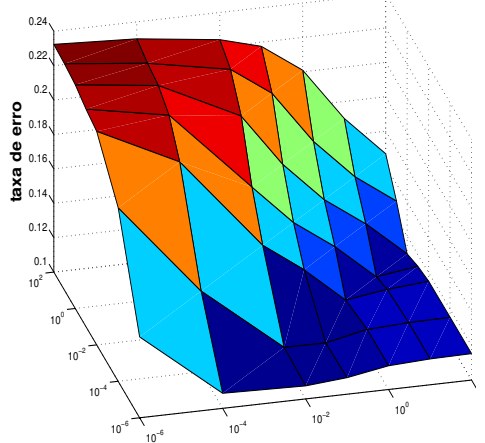

(d) symKNN-HM

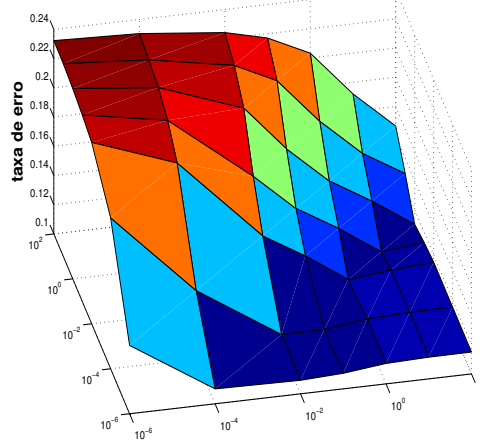

(g) symKNN-LLE

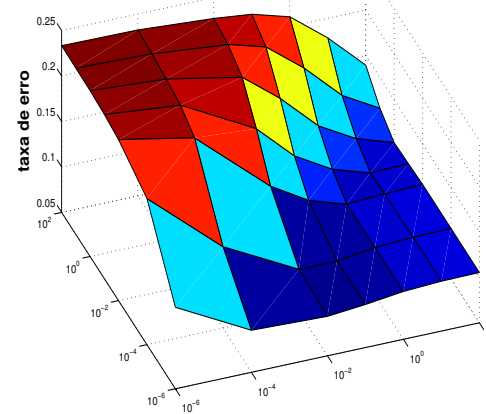

(b) mutKNN-RBF

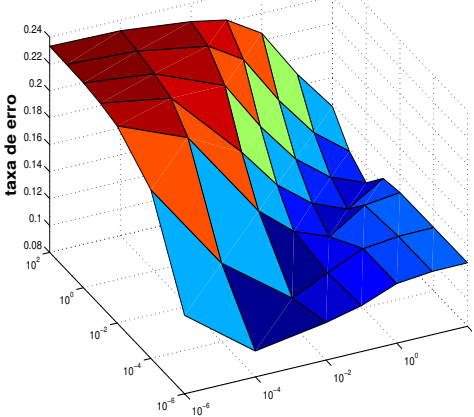

(e) mutKNN-HM

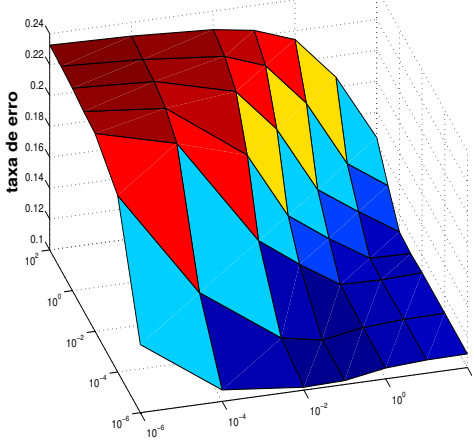

(h) mutKNN-LLE

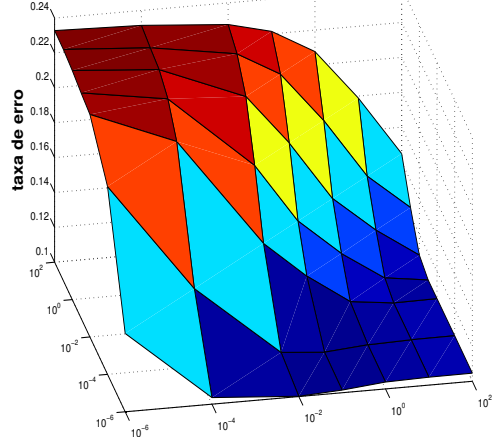

(c) symFKNN-RBF

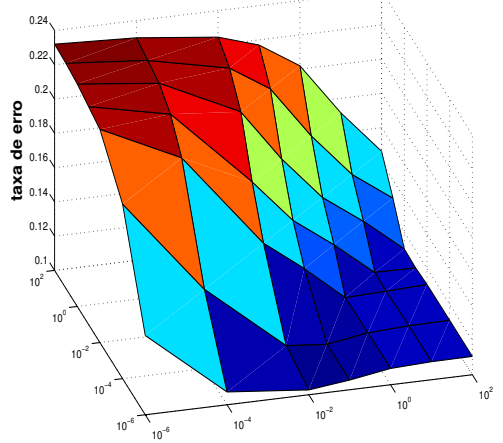

(f) symFKNN-HM

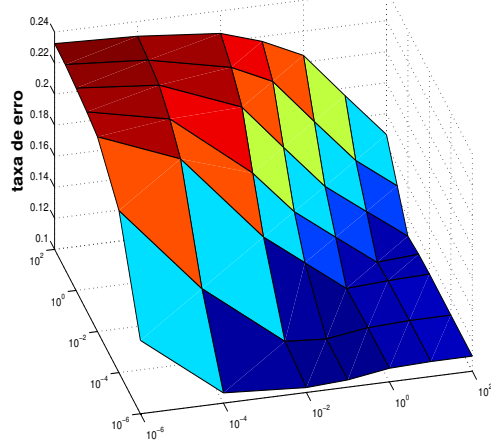

(i) symFKNN-LLE

Figura 7.10: Superfícies de erro do algoritmo LapRLS na base de dados DIGIT-1 para as partições de 10 exemplos rotulados. 


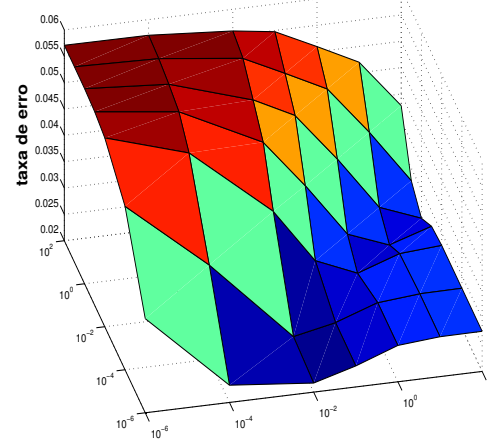

(a) symKNN-RBF

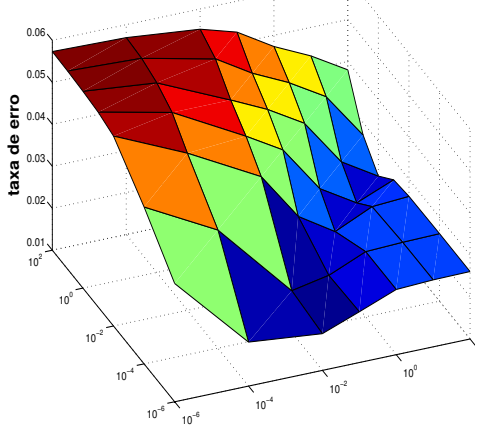

(d) symKNN-HM

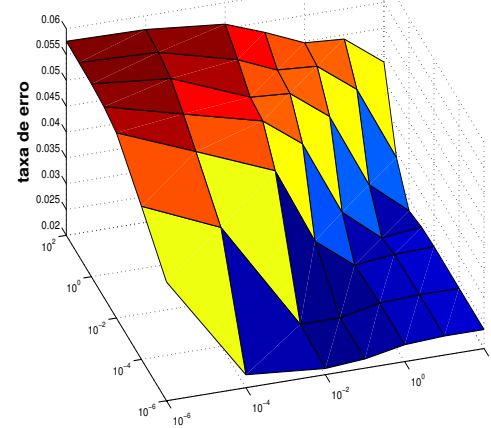

(g) symKNN-LLE

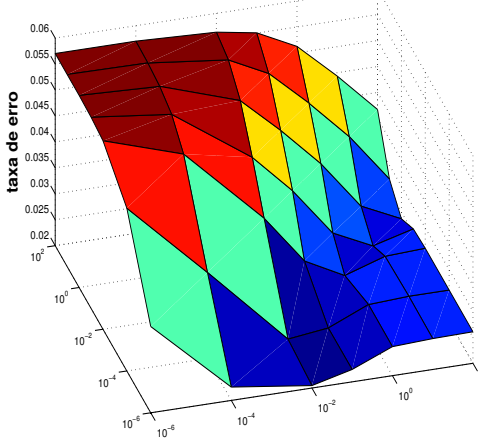

(b) mutKNN-RBF

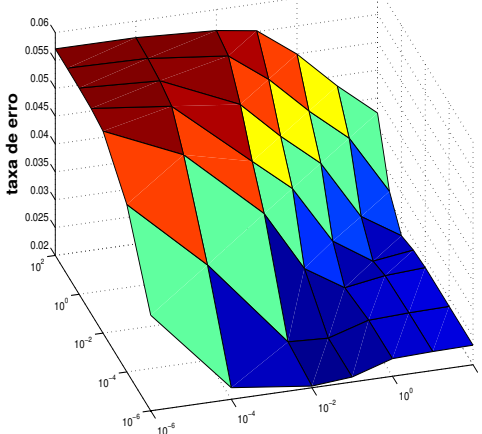

(e) mutKNN-HM

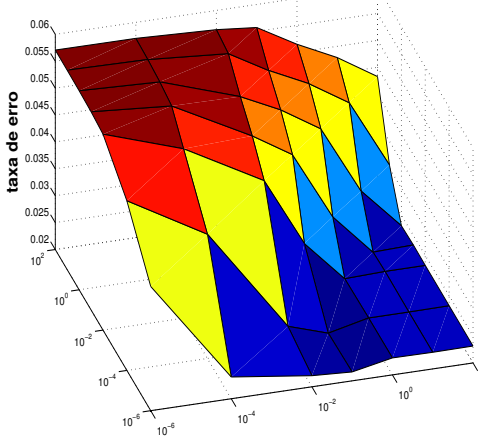

(h) mutKNN-LLE

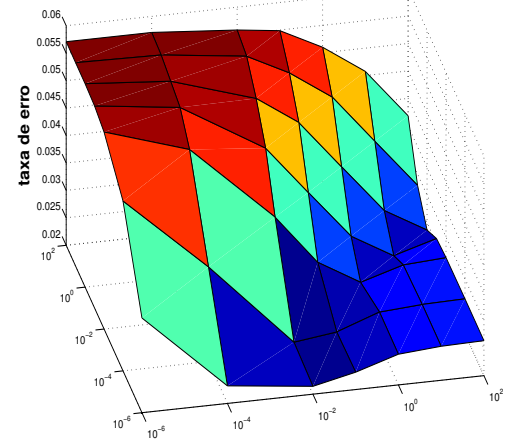

(c) symFKNN-RBF

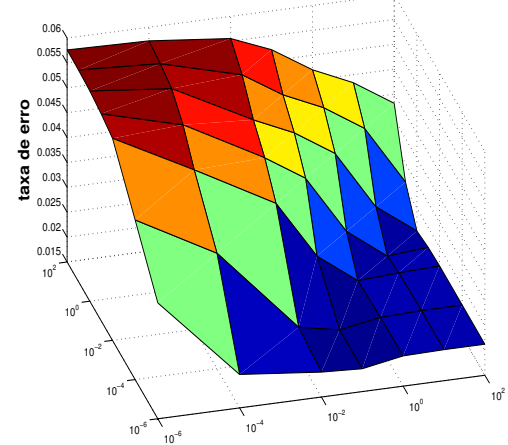

(f) symFKNN-HM

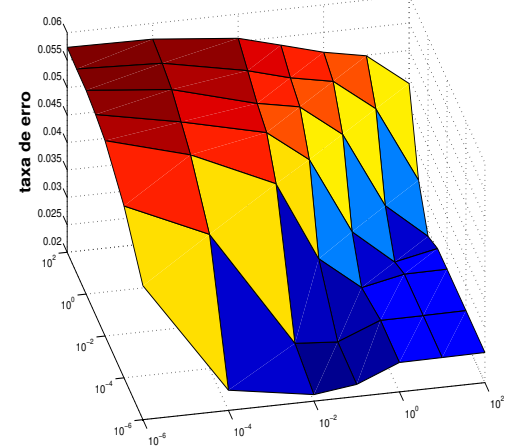

(i) SymFKNN-LLE

Figura 7.11: Superfícies de erro do algoritmo LapRLS na base de dados DIGIT-1 para as partições de 100 exemplos rotulados. 


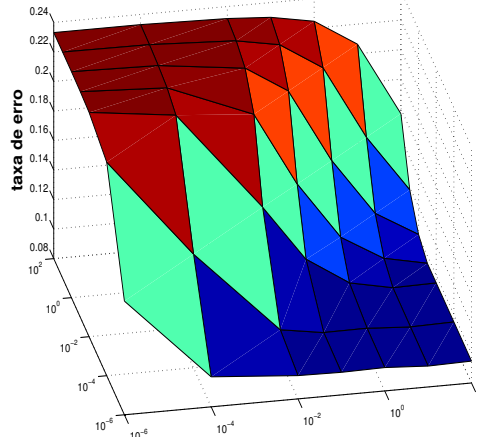

(a) symKNN-RBF

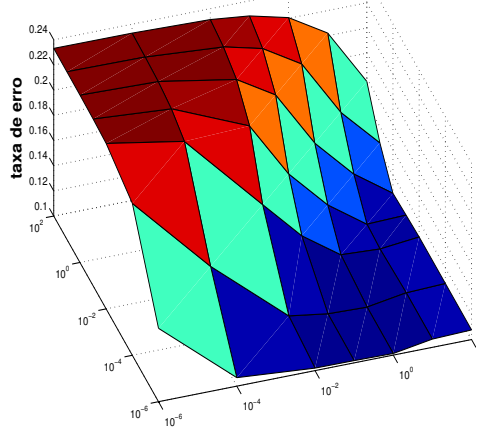

(d) symKNN-HM

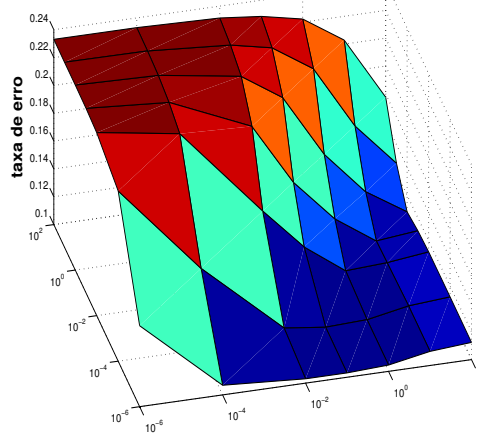

(g) symKNN-LLE

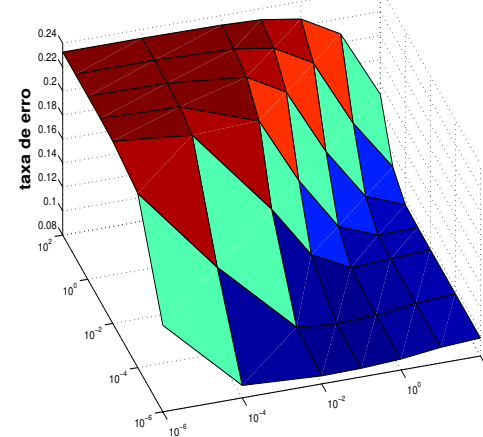

(b) mutKNN-RBF

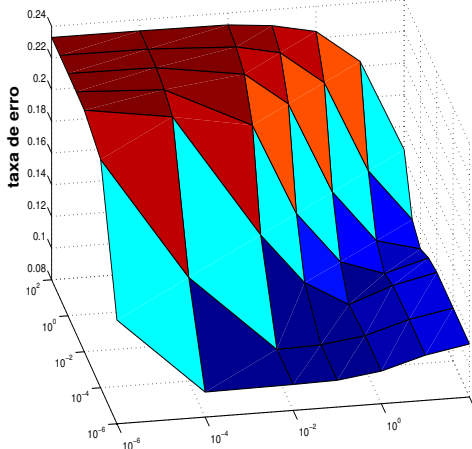

(e) mutKNN-HM

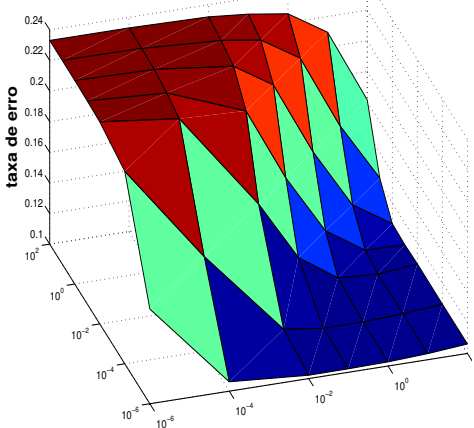

(h) mutKNN-LLE

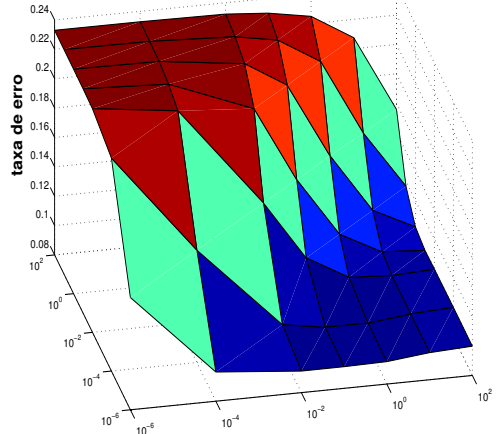

(c) symFKNN-RBF

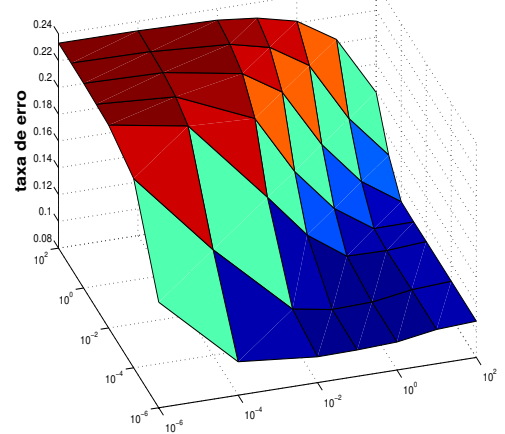

(f) symFKNN-HM

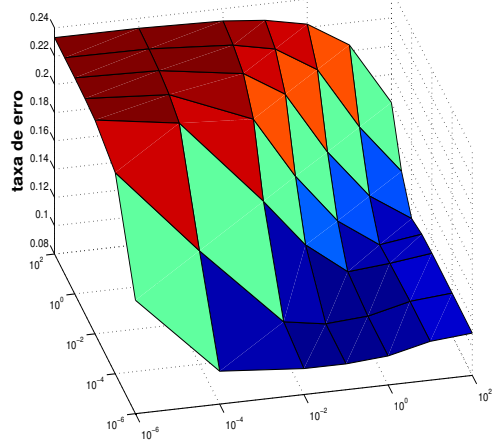

(i) symFKNN-LLE

Figura 7.12: Superfícies de erro do algoritmo LapSVM na base de dados DIGIT-1 para as partições de 10 exemplos rotulados. 


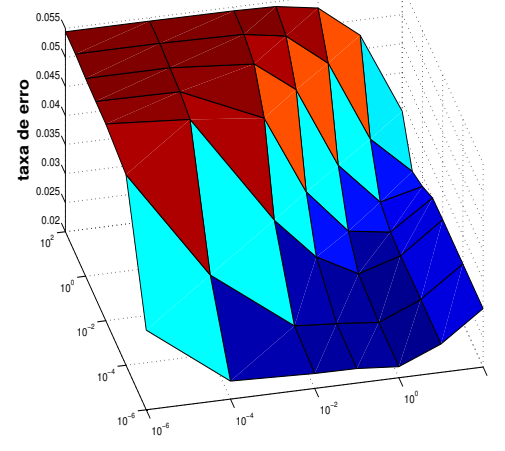

(a) symKNN-RBF

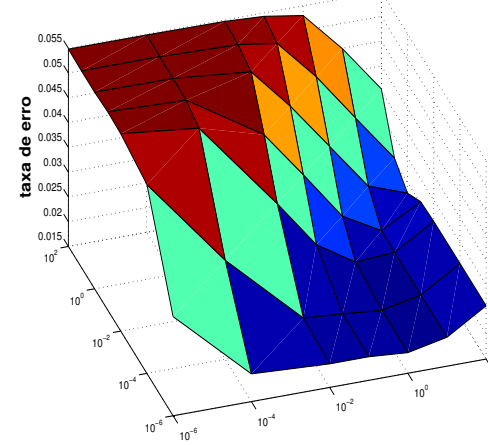

(d) symKNN-HM

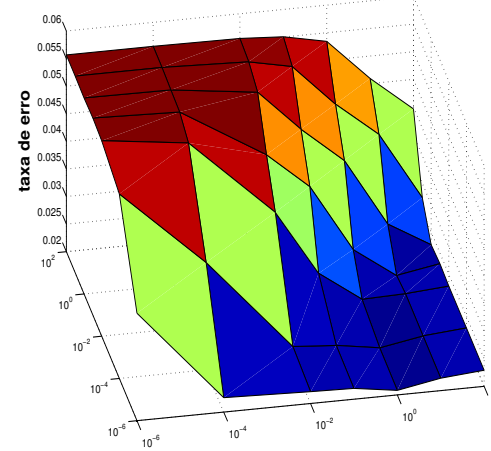

(g) symKNN-LLE

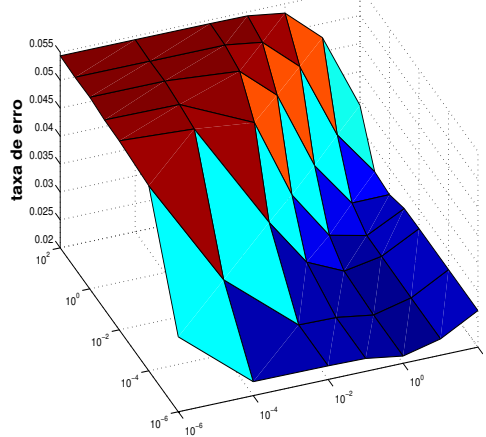

(b) mutKNN-RBF

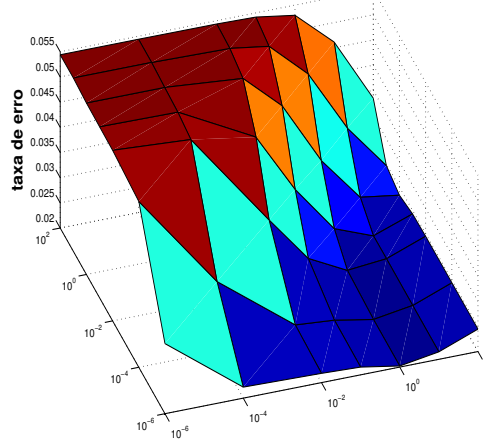

(e) mutKNN-HM

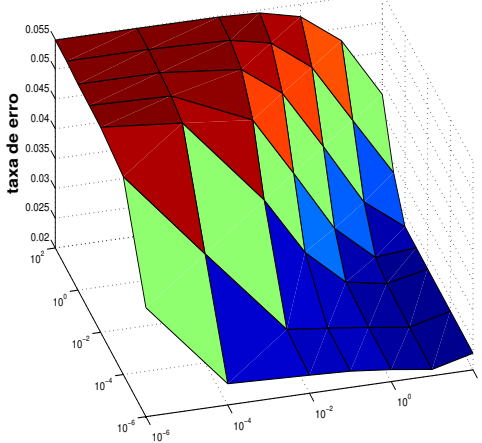

(h) mutKNN-LLE

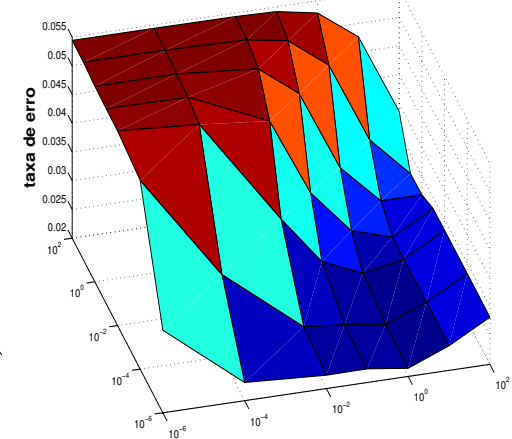

(c) symFKNN-RBF

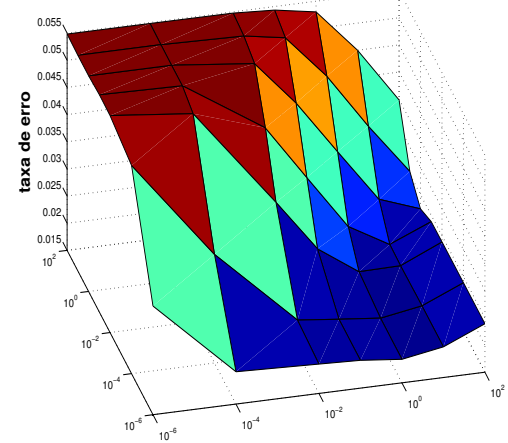

(f) symFKNN-HM

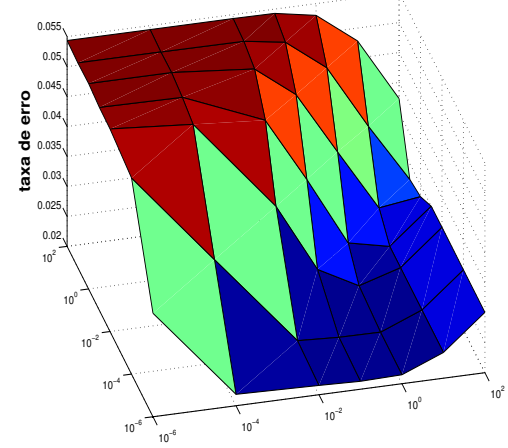

(i) SymFKNN-LLE

Figura 7.13: Superfícies de erro do algoritmo LapSVM na base de dados DIGIT-1 para as partições de 100 exemplos rotulados. 


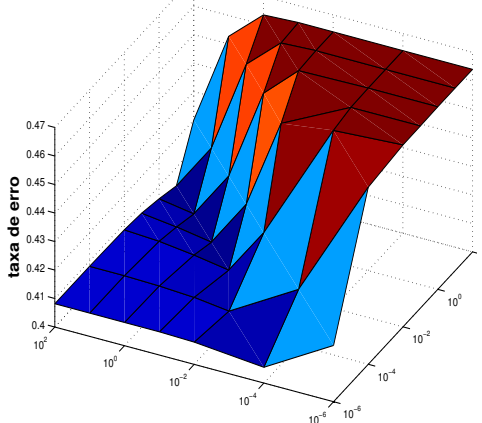

(a) symKNN-RBF

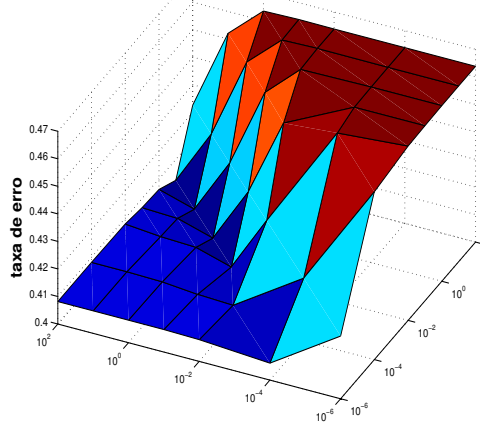

(d) symKNN-HM

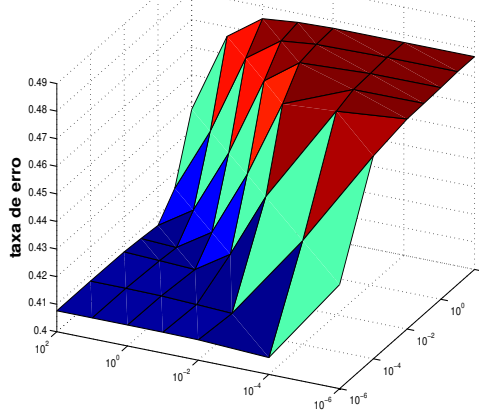

(g) symKNN-LLE

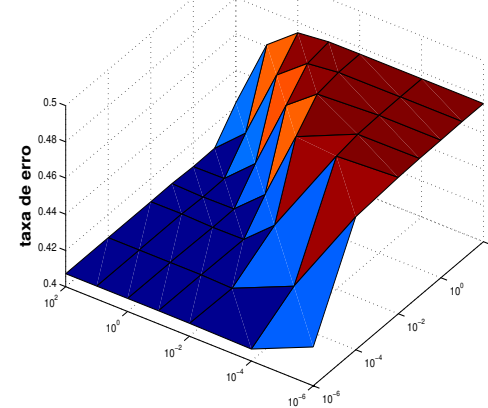

(b) mutKNN-RBF

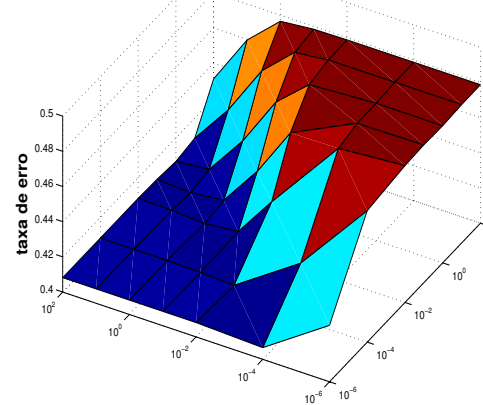

(e) mutKNN-HM

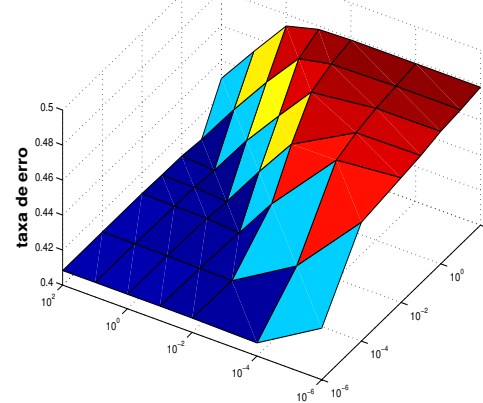

(h) mutKNN-LLE

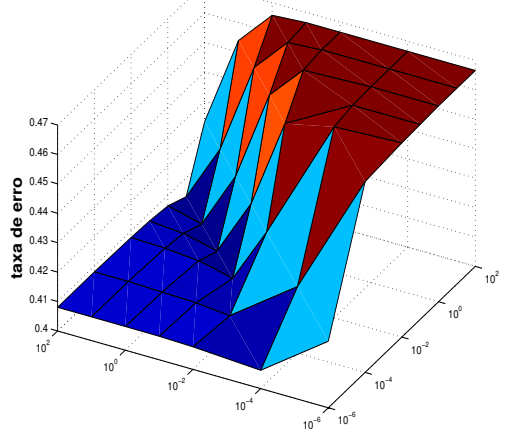

(c) symFKNN-RBF

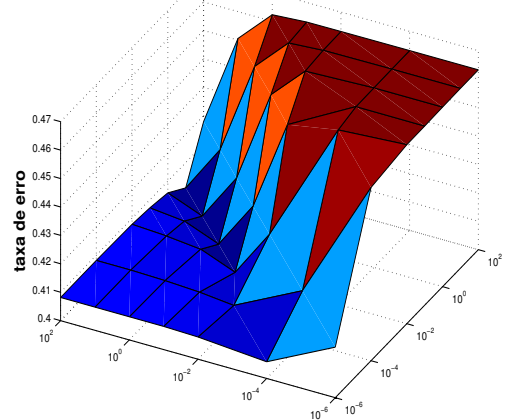

(f) symFKNN-HM

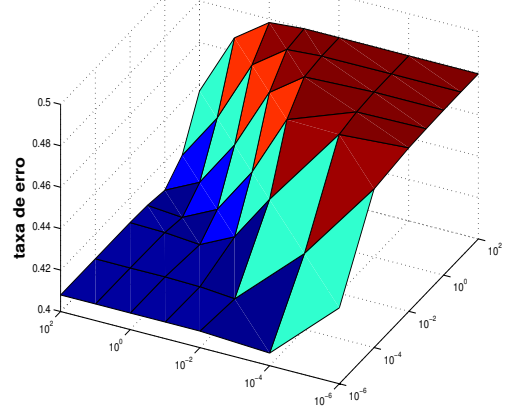

(i) SymFKNN-LLE

Figura 7.14: Superfícies de erro do algoritmo LapRLS na base de dados G-241C para as partições de 10 exemplos rotulados. 


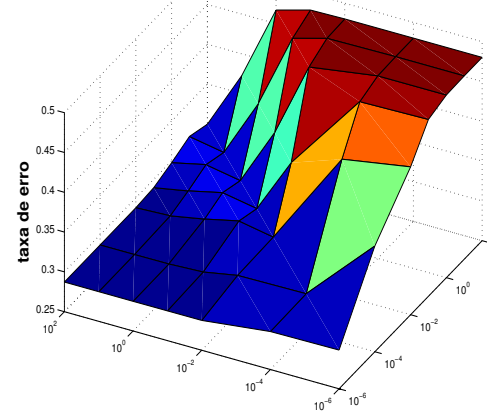

(a) symKNN-RBF

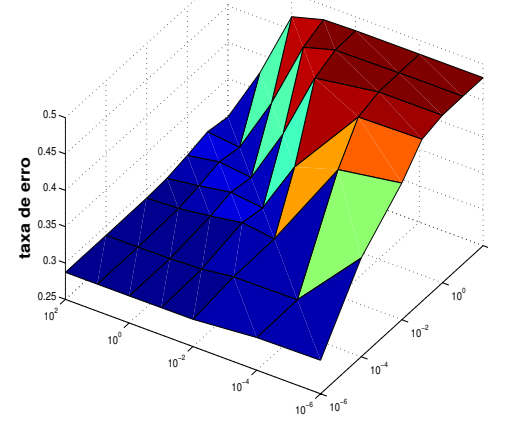

(d) symKNN-HM

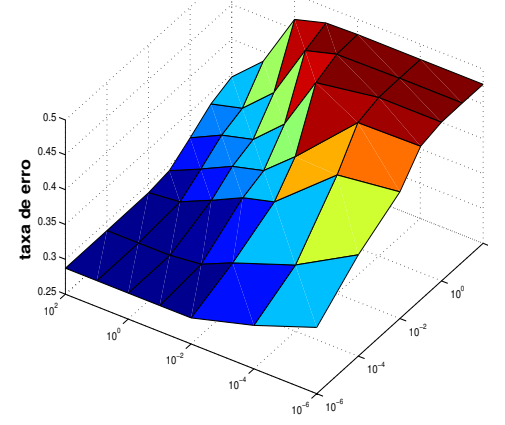

(g) symKNN-LLE

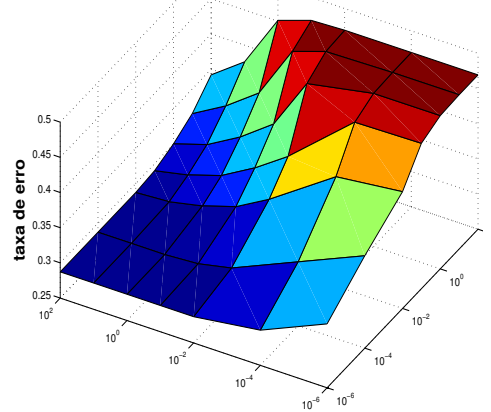

(b) mutKNN-RBF

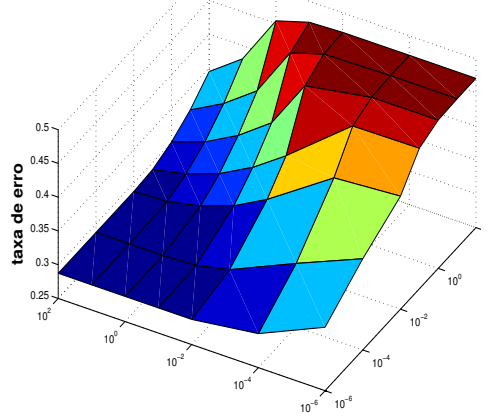

(e) mutKNN-HM

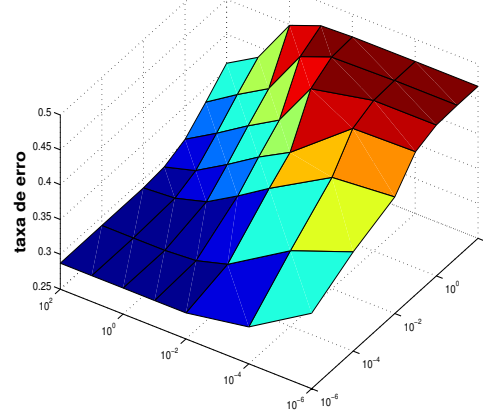

(h) mutKNN-LLE

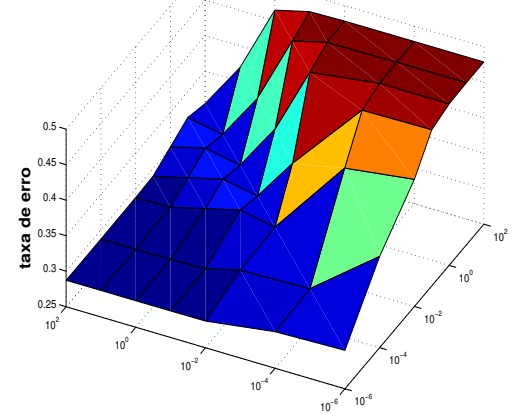

(c) symFKNN-RBF

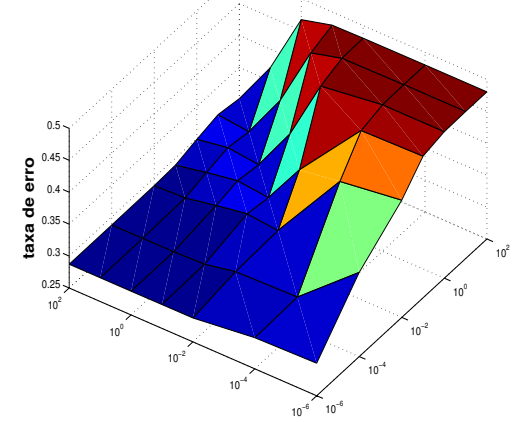

(f) symFKNN-HM

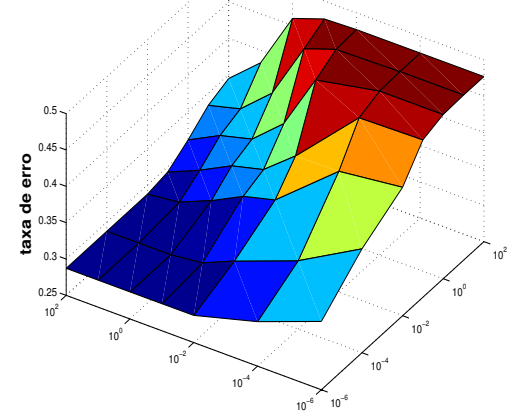

(i) symFKNN-LLE

Figura 7.15: Superfícies de erro do algoritmo LapRLS na base de dados G-241C para as partições de 100 exemplos rotulados. 


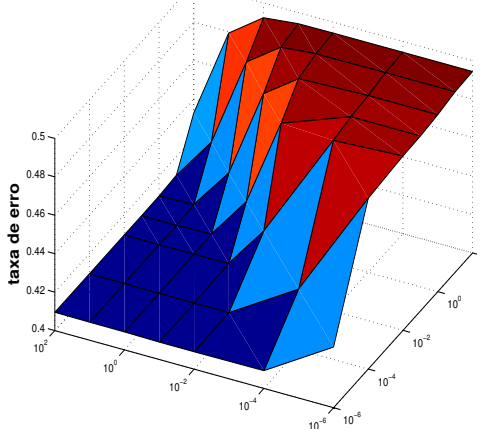

(a) symKNN-RBF

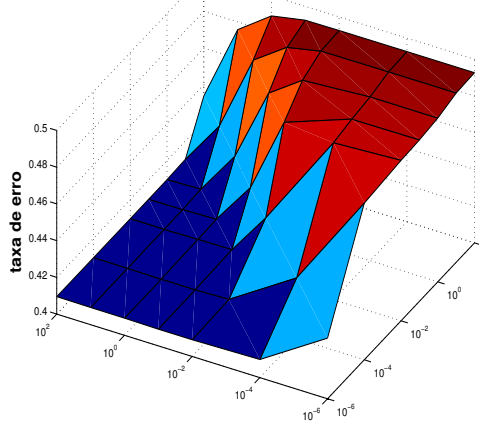

(d) symKNN-HM

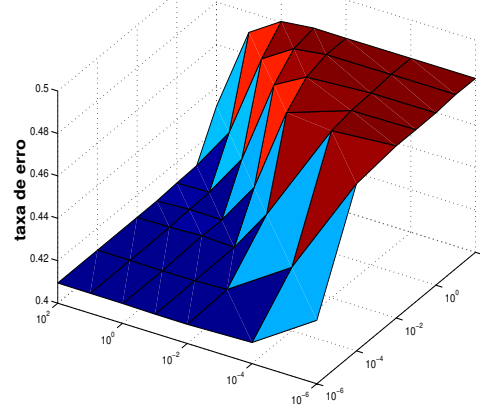

(g) symKNN-LLE

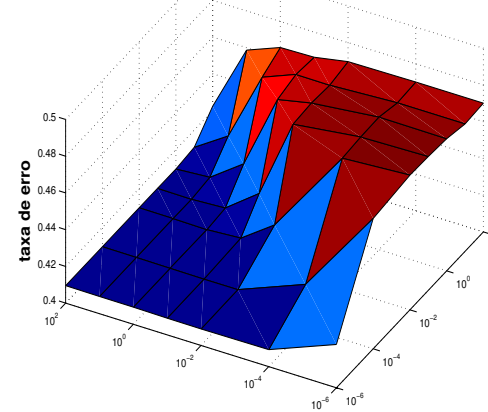

(b) mutKNN-RBF

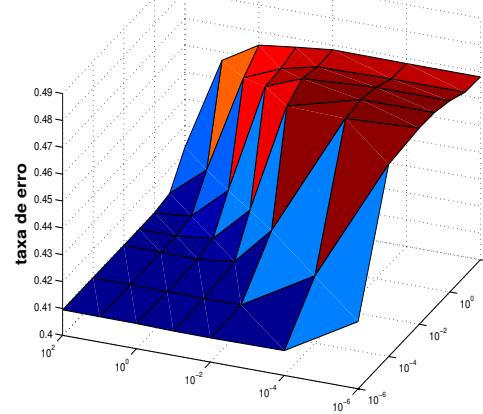

(e) mutKNN-HM

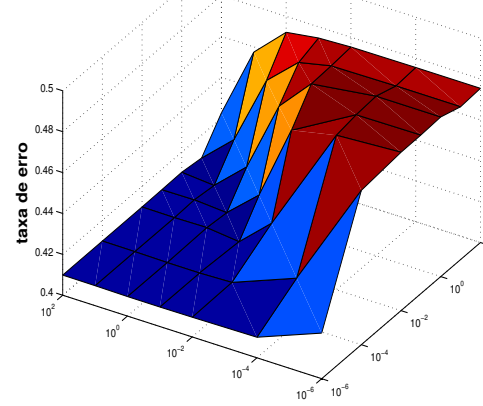

(h) mutKNN-LLE

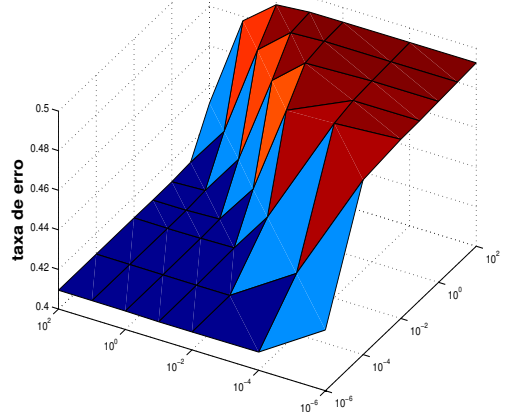

(c) symFKNN-RBF

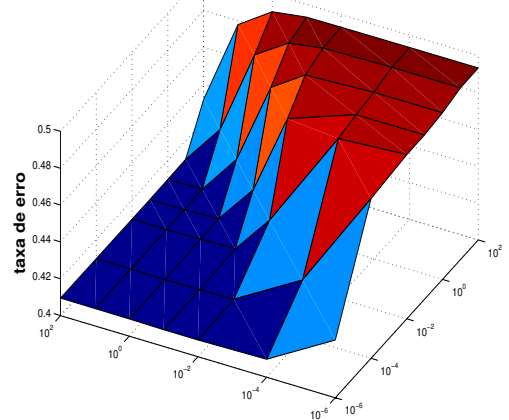

(f) symFKNN-HM

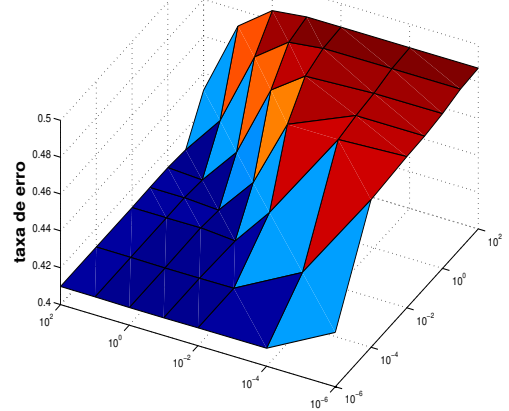

(i) SymFKNN-LLE

Figura 7.16: Superfícies de erro do algoritmo LapSVM na base de dados G-241C para as partições de 10 exemplos rotulados. 


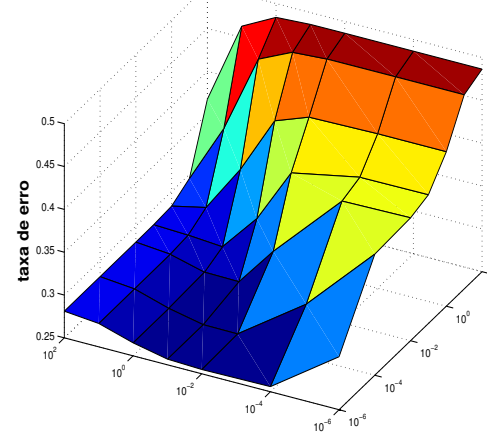

(a) symKNN-RBF

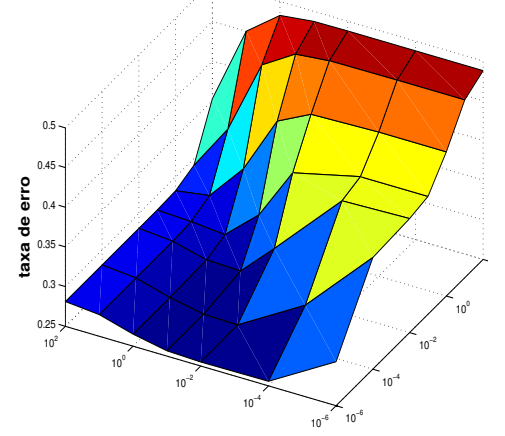

(d) symKNN-HM

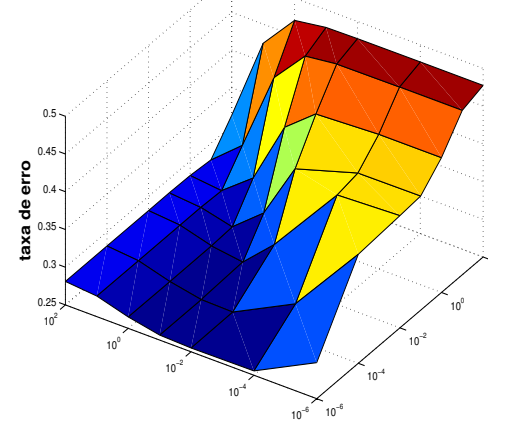

(g) symKNN-LLE

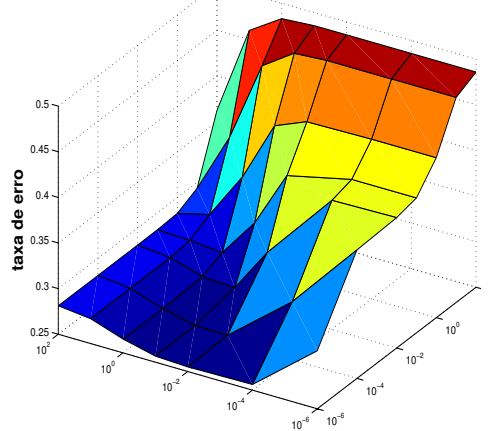

(b) mutKNN-RBF

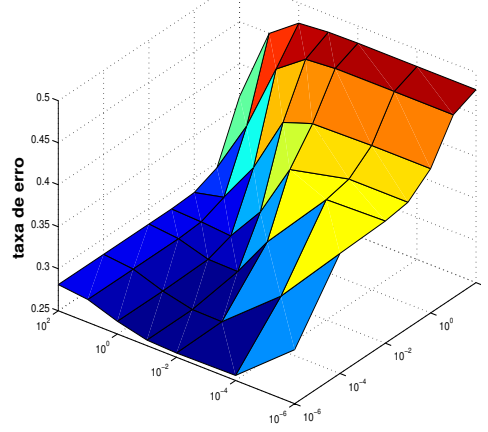

(e) mutKNN-HM

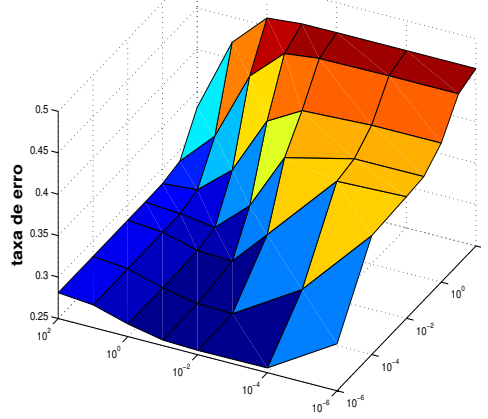

(h) mutKNN-LLE

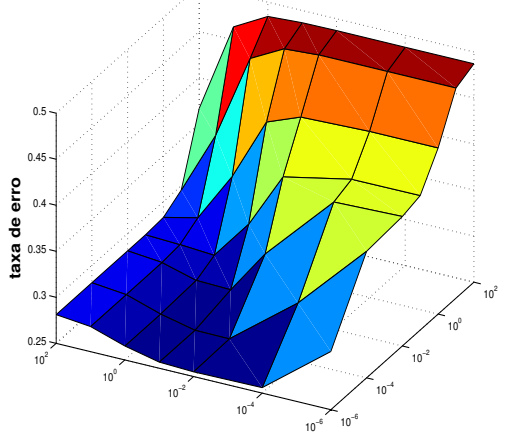

(c) symFKNN-RBF

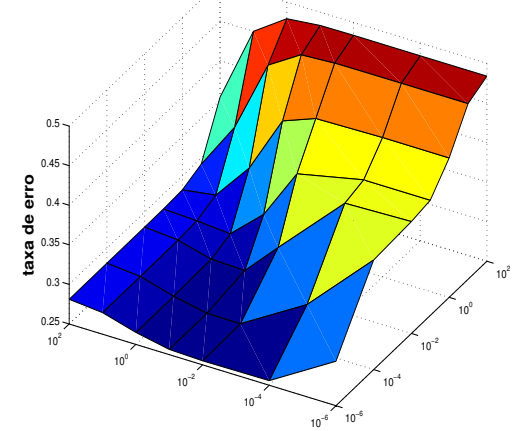

(f) symFKNN-HM

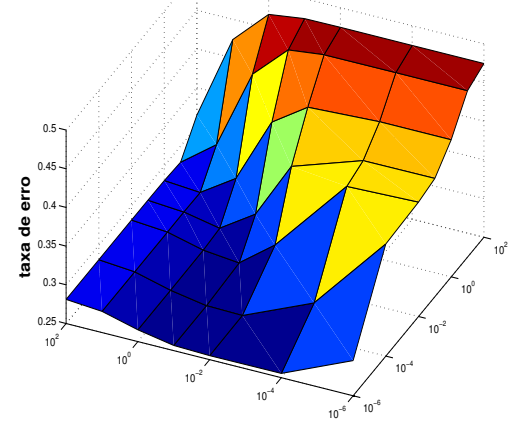

(i) symFKNN-LLE

Figura 7.17: Superfícies de erro do algoritmo LapSVM na base de dados G-241C para as partições de 100 exemplos rotulados. 


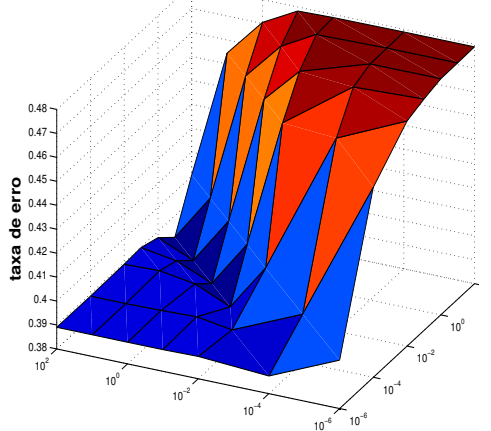

(a) symKNN-RBF

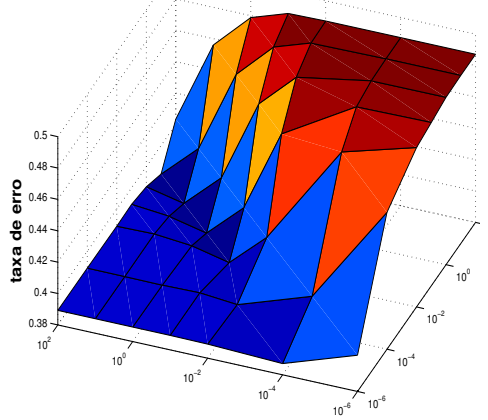

(d) symKNN-HM

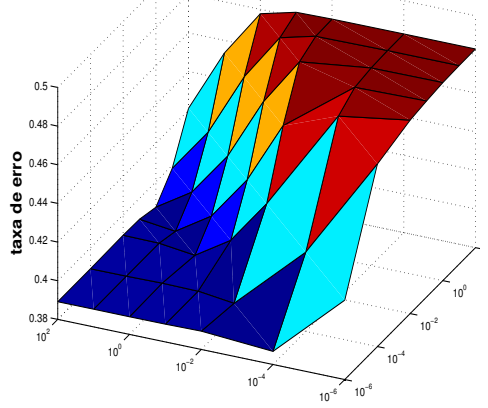

(g) symKNN-LLE

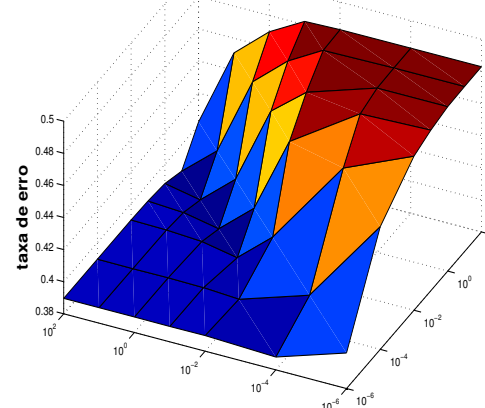

(b) mutKNN-RBF

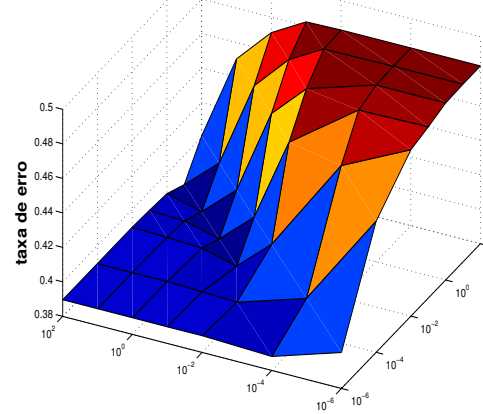

(e) mutKNN-HM

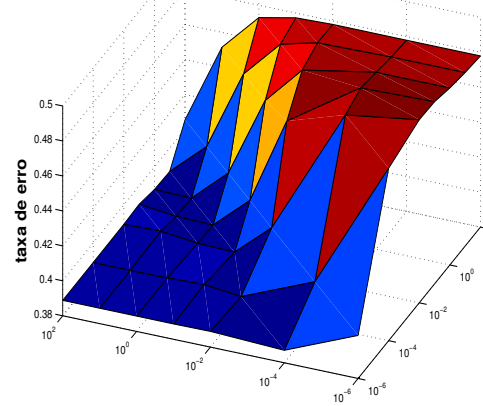

(h) mutKNN-LLE

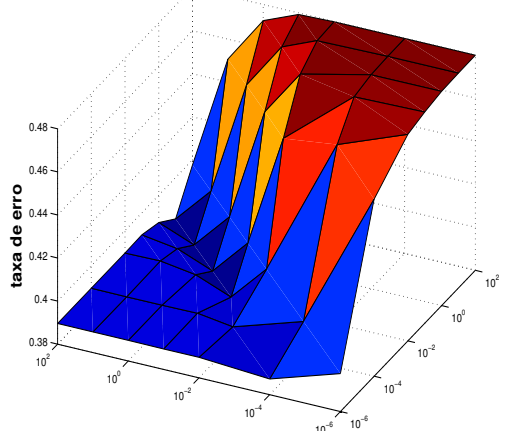

(c) symFKNN-RBF

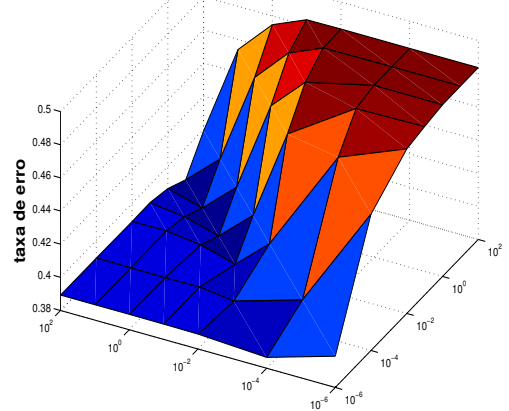

(f) symFKNN-HM

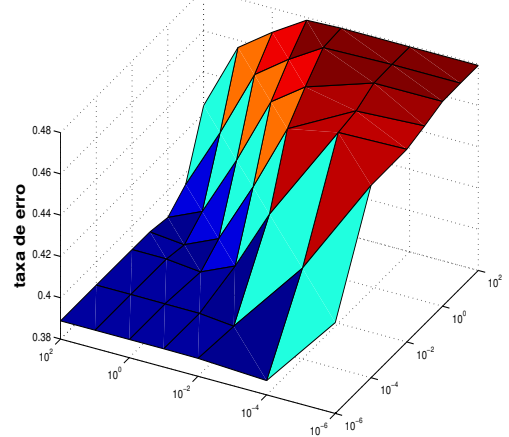

(i) symFKNN-LLE

Figura 7.18: Superfícies de erro do algoritmo LapRLS na base de dados G-241N para as partições de 10 exemplos rotulados. 


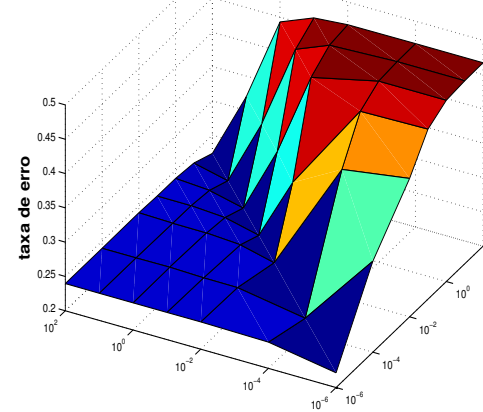

(a) symKNN-RBF

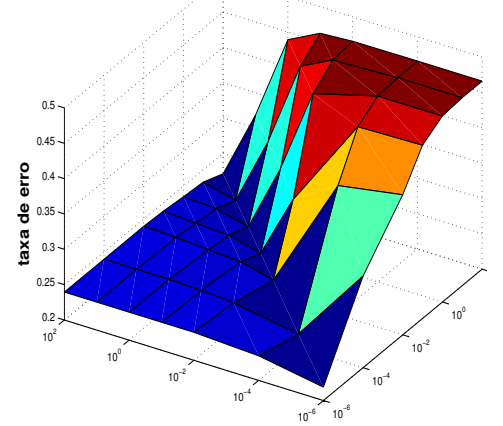

(d) symKNN-HM

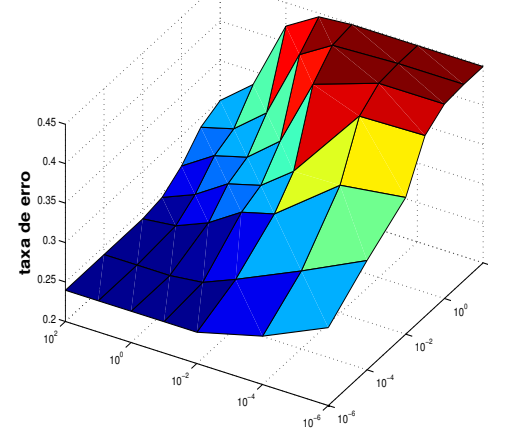

(g) symKNN-LLE

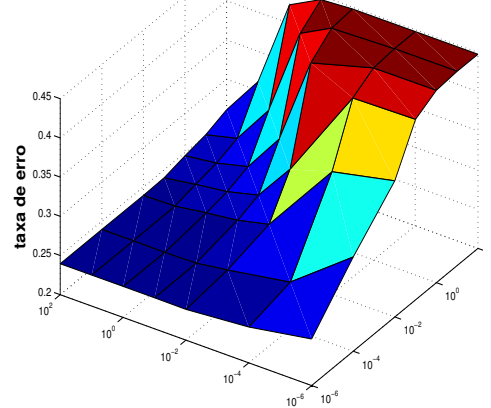

(b) mutKNN-RBF

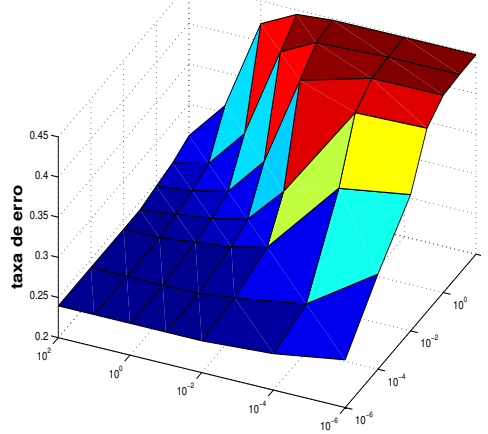

(e) mutKNN-HM

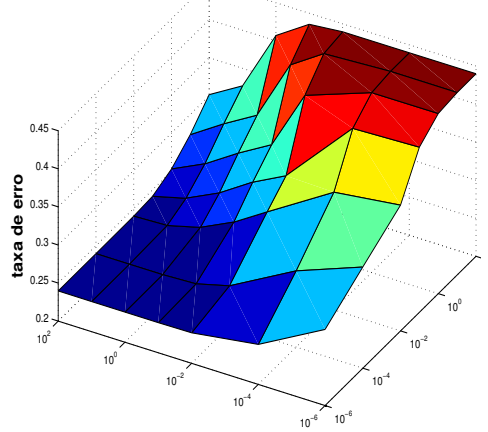

(h) mutKNN-LLE

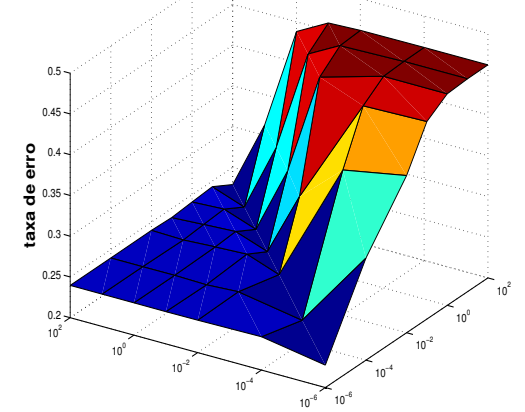

(c) symFKNN-RBF

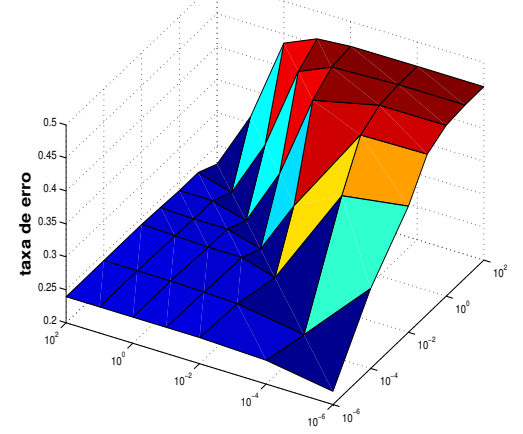

(f) symFKNN-HM

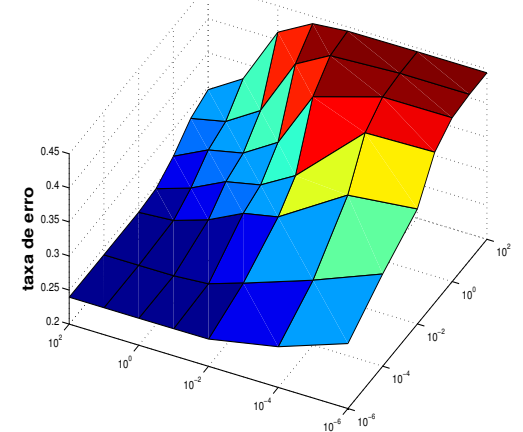

(i) symFKNN-LLE

Figura 7.19: Superfícies de erro do algoritmo LapRLS na base de dados G-241N para as partições de 100 exemplos rotulados. 


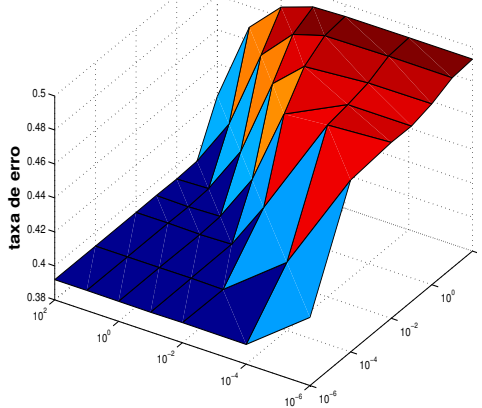

(a) symKNN-RBF

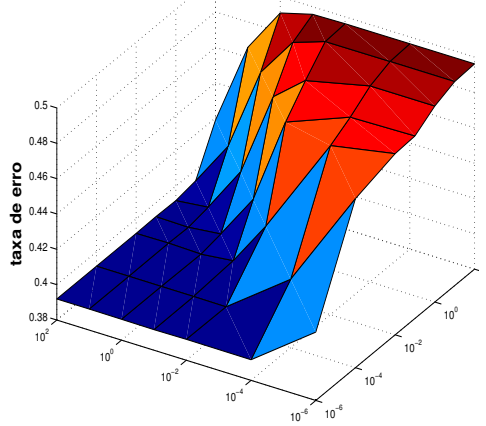

(d) symKNN-HM

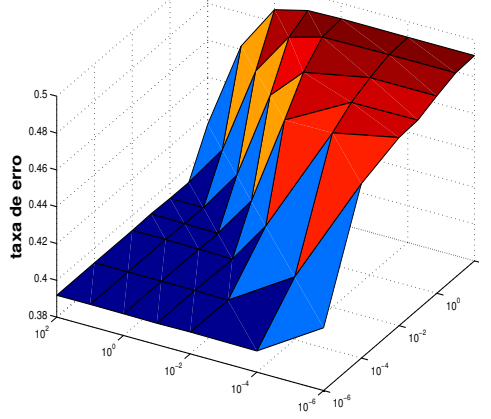

(g) symKNN-LLE

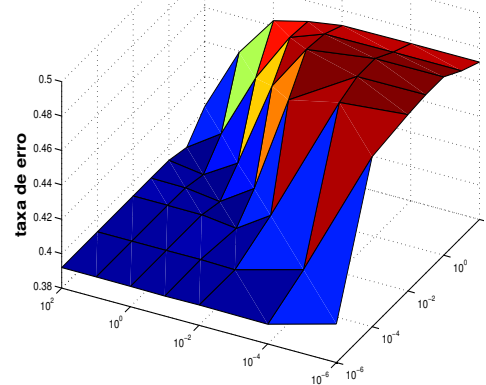

(b) mutKNN-RBF

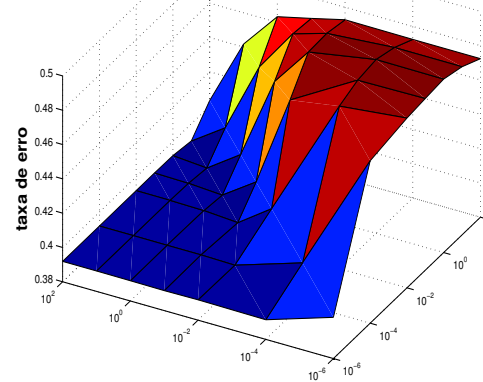

(e) mutKNN-HM

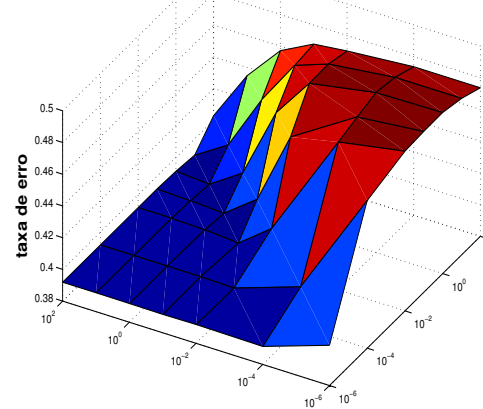

(h) mutKNN-LLE

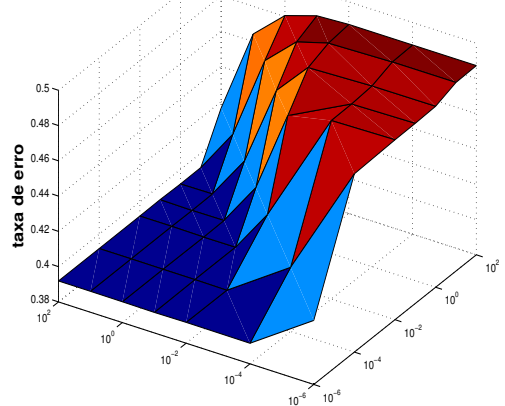

(c) symFKNN-RBF

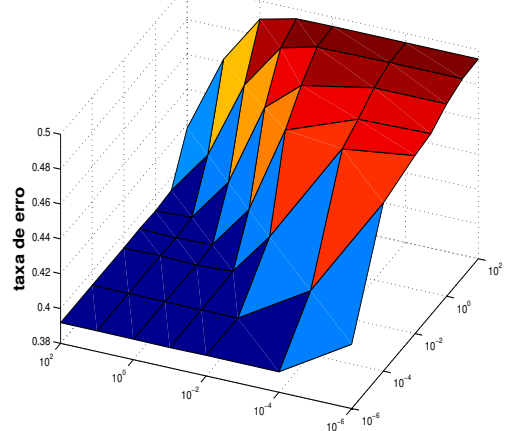

(f) symFKNN-HM

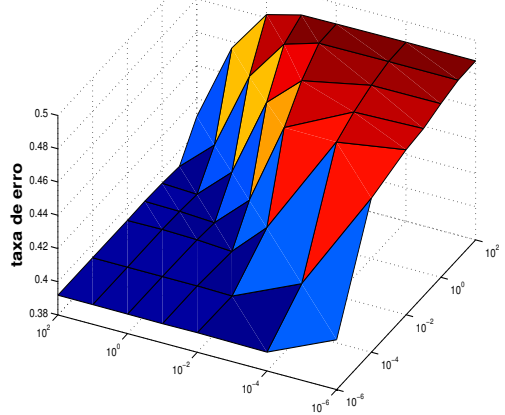

(i) SymFKNN-LLE

Figura 7.20: Superfícies de erro do algoritmo LapSVM na base de dados G-241N para as partições de 10 exemplos rotulados. 


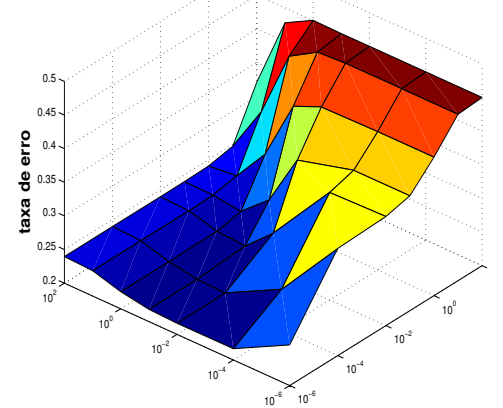

(a) symKNN-RBF

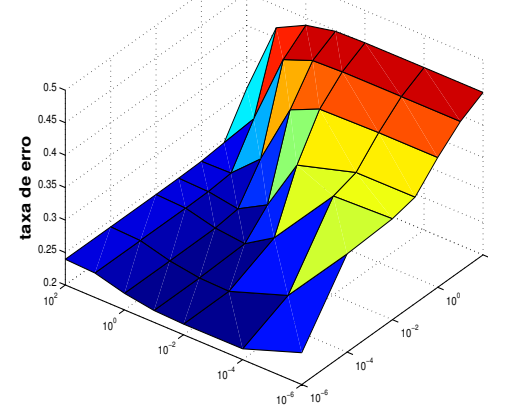

(d) symKNN-HM

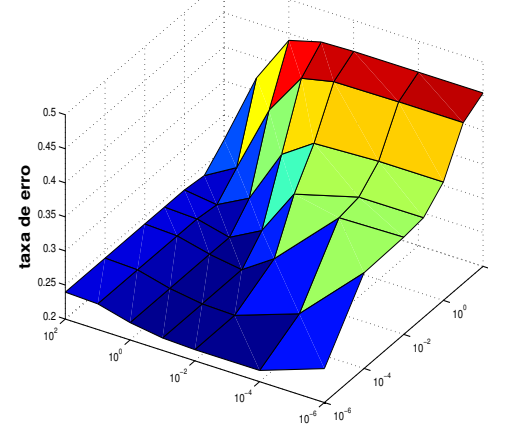

(g) symKNN-LLE

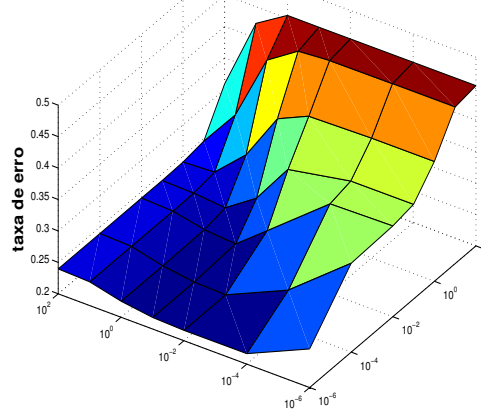

(b) mutKNN-RBF

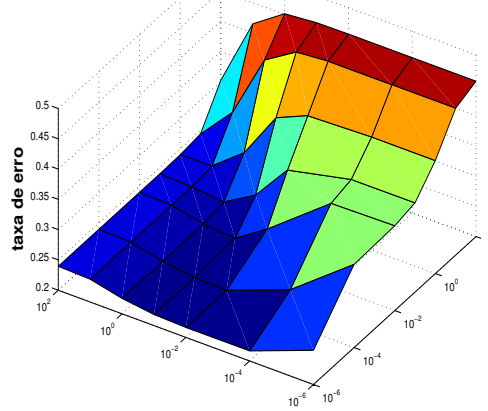

(e) mutKNN-HM

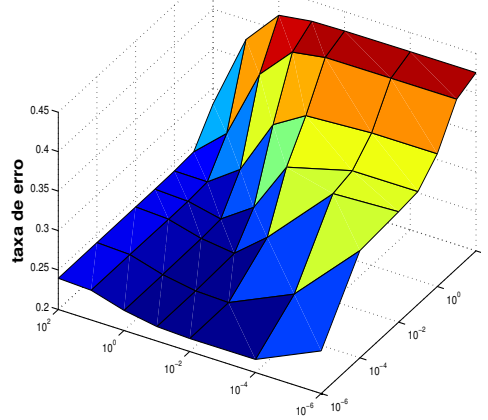

(h) mutKNN-LLE

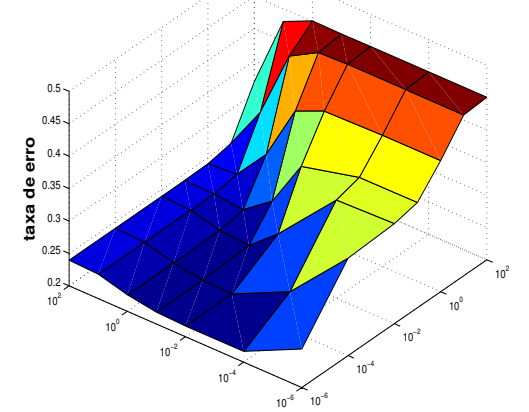

(c) symFKNN-RBF

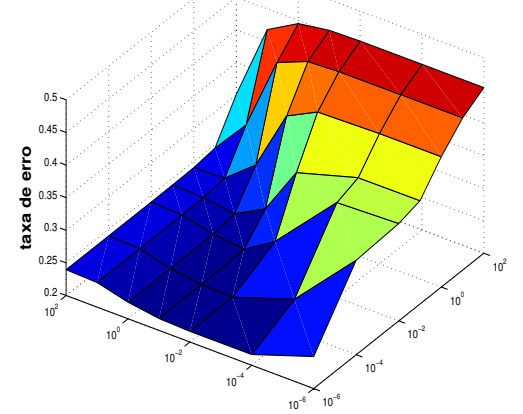

(f) symFKNN-HM

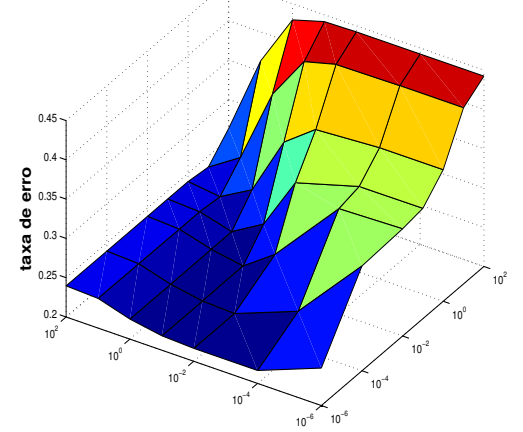

(i) SymFKNN-LLE

Figura 7.21: Superfícies de erro do algoritmo LapSVM na base de dados G-241N para as partições de 100 exemplos rotulados. 


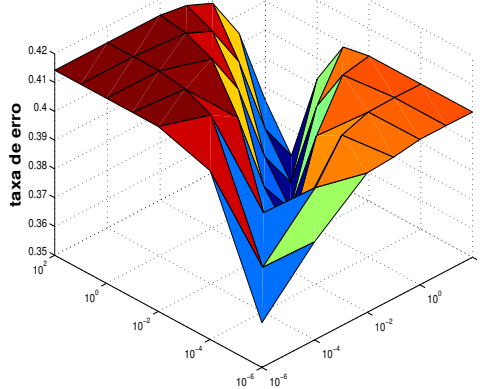

(a) symKNN-RBF

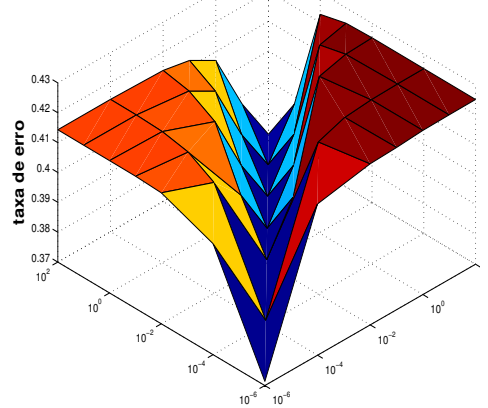

(d) symKNN-HM

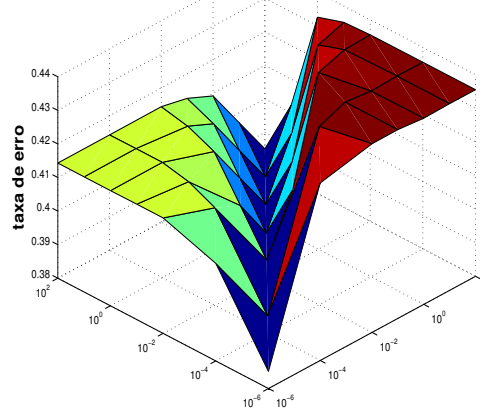

(g) symKNN-LLE

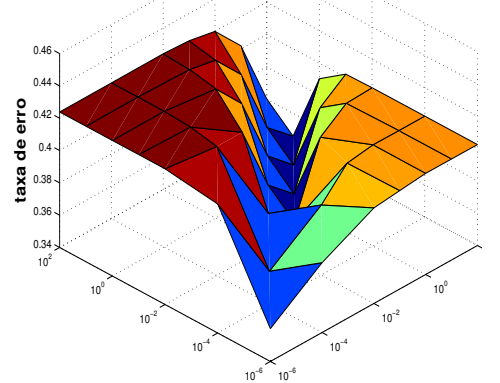

(b) mutKNN-RBF

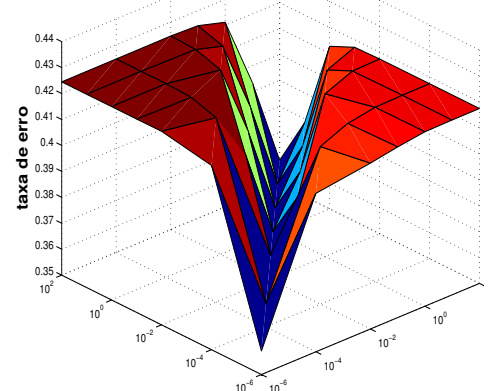

(e) mutKNN-HM

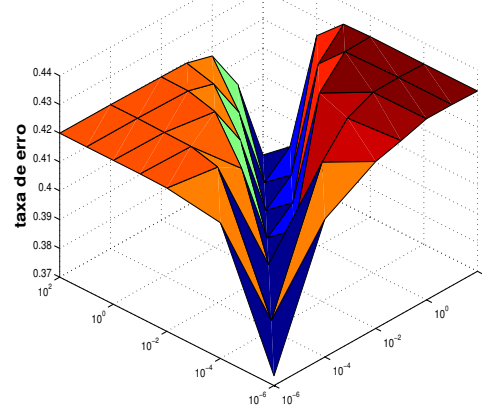

(h) mutKNN-LLE

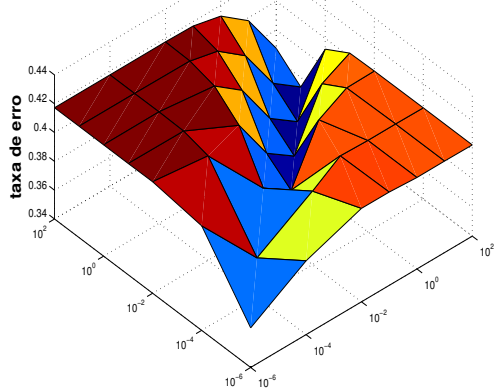

(c) symFKNN-RBF

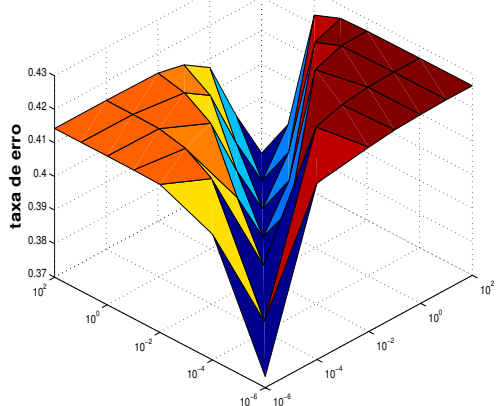

(f) symFKNN-HM

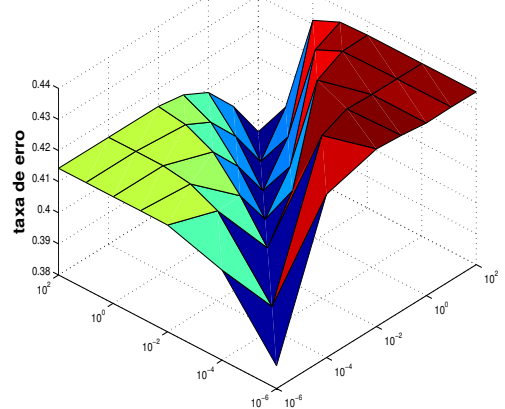

(i) symFKNN-LLE

Figura 7.22: Superfícies de erro do algoritmo LapRLS na base de dados TEXT para as partições de 10 exemplos rotulados. 


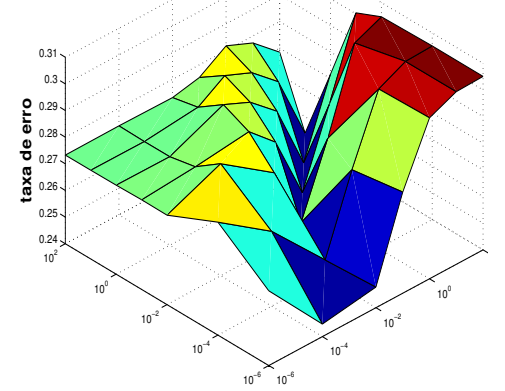

(a) symKNN-RBF

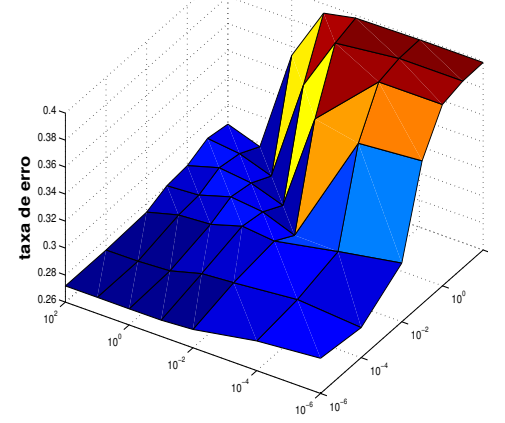

(d) symKNN-HM

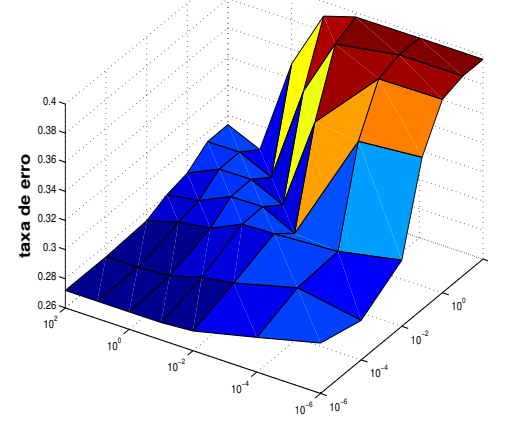

(g) symKNN-LLE

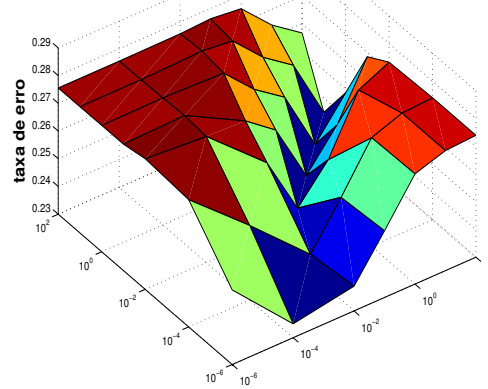

(b) mutKNN-RBF

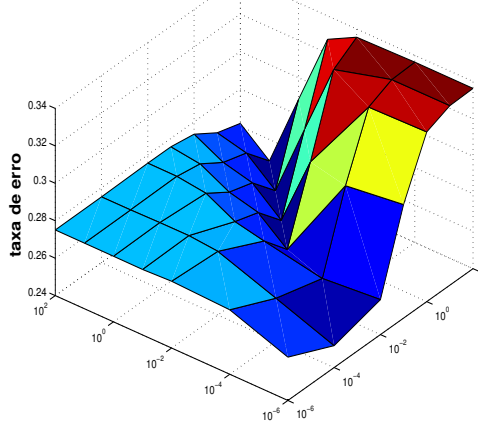

(e) mutKNN-HM

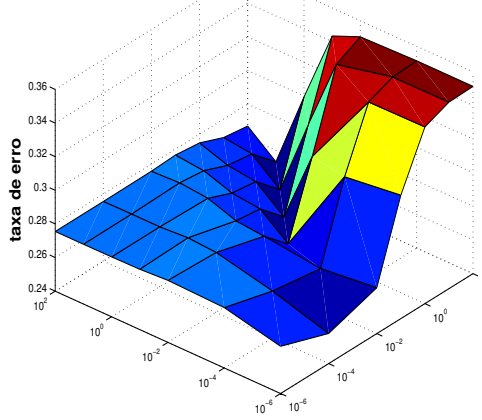

(h) mutKNN-LLE

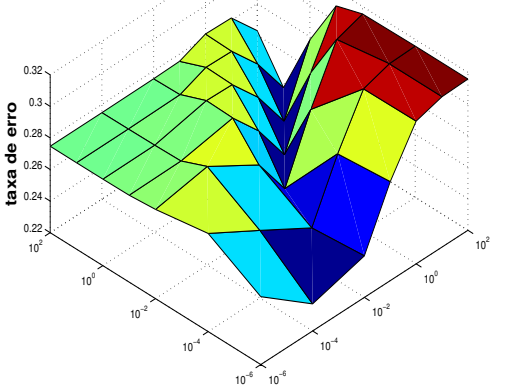

(c) symFKNN-RBF

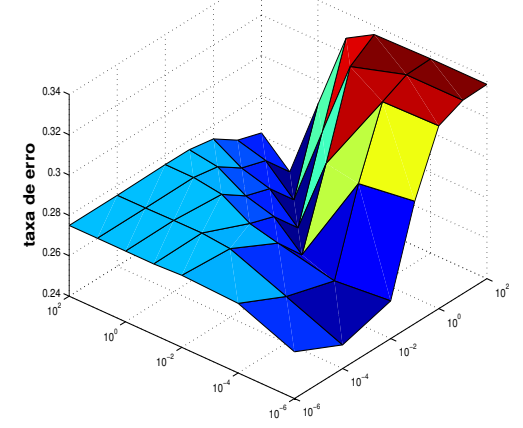

(f) symFKNN-HM

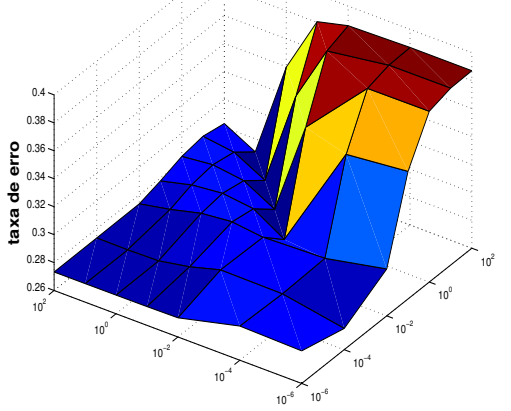

(i) symFKNN-LLE

Figura 7.23: Superfícies de erro do algoritmo LapRLS na base de dados TEXT para as partições de 100 exemplos rotulados. 


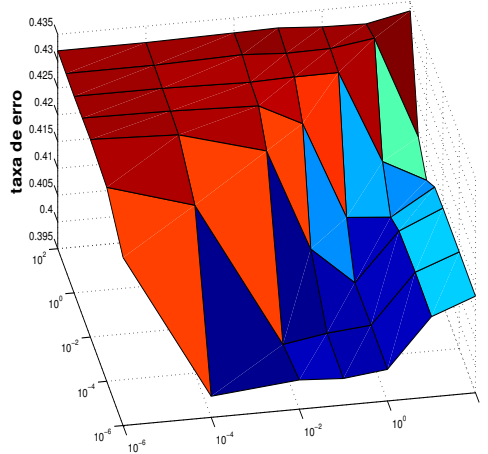

(a) symKNN-RBF

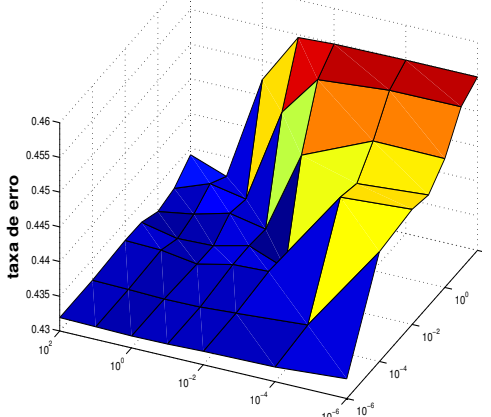

(d) symKNN-HM

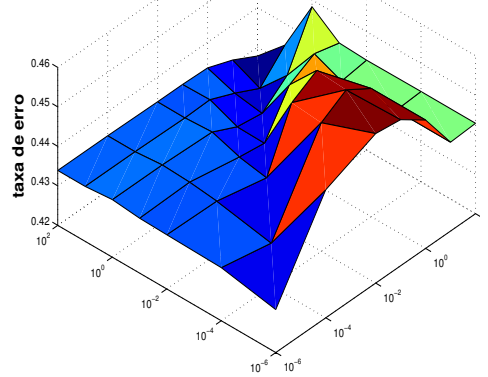

(g) symKNN-LLE

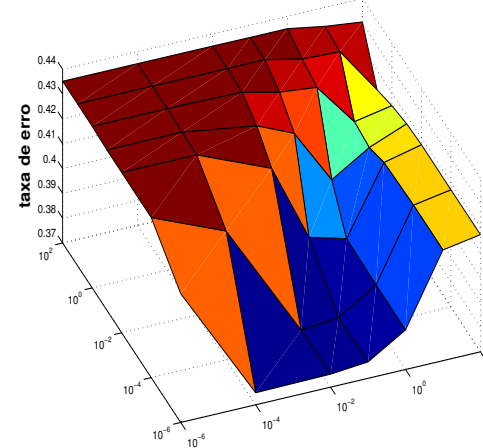

(b) mutKNN-RBF

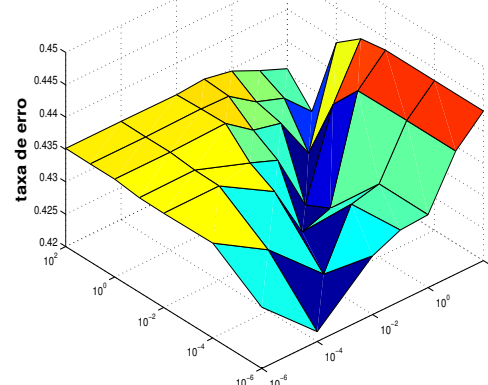

(e) mutKNN-HM

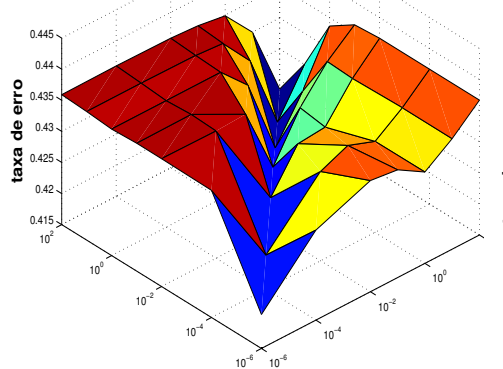

(h) mutKNN-LLE

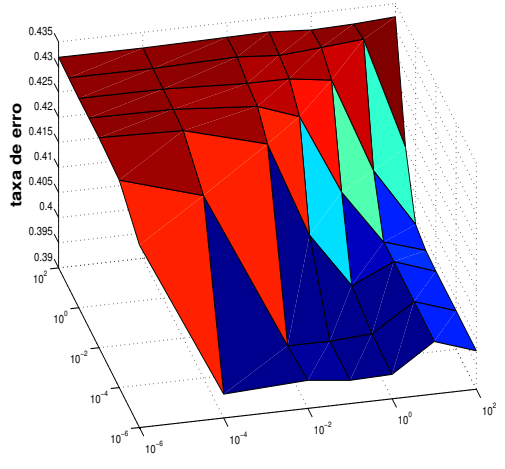

(c) symFKNN-RBF

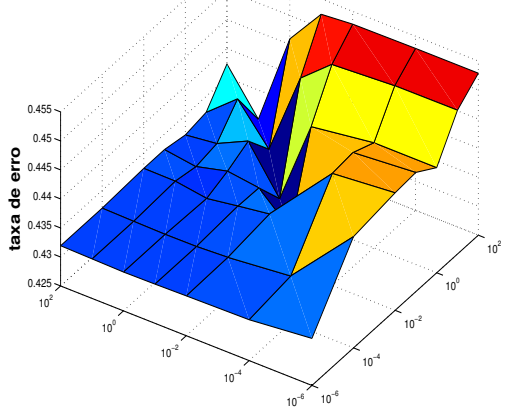

(f) symFKNN-HM

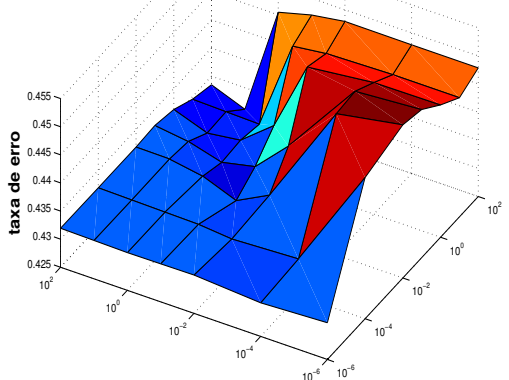

(i) SymFKNN-LLE

Figura 7.24: Superfícies de erro do algoritmo LapSVM na base de dados TEXT para as partições de 10 exemplos rotulados. 


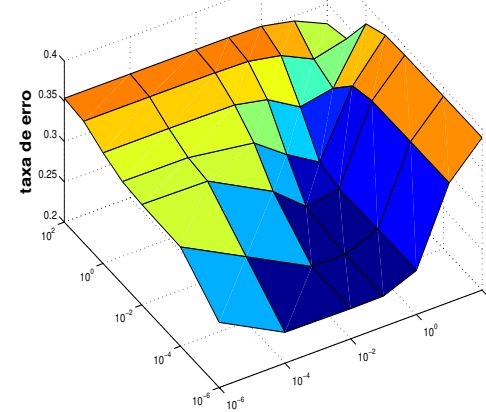

(a) symKNN-RBF

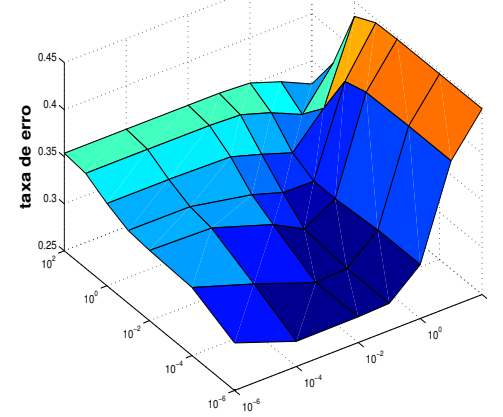

(d) symKNN-HM

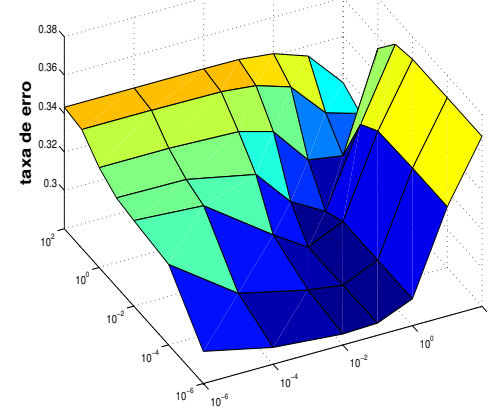

(g) symKNN-LLE

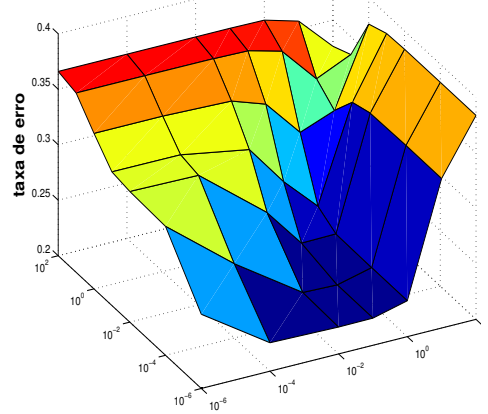

(b) mutKNN-RBF

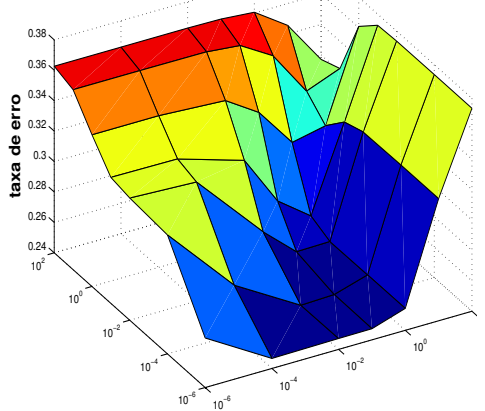

(e) mutKNN-HM

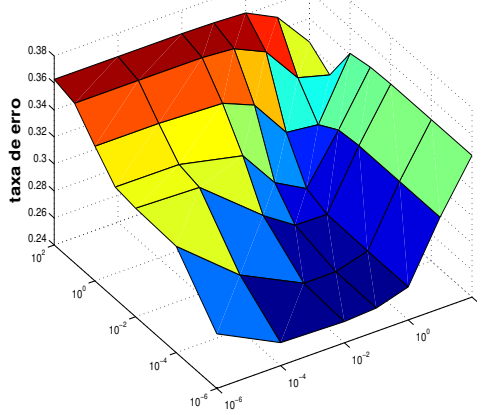

(h) mutKNN-LLE

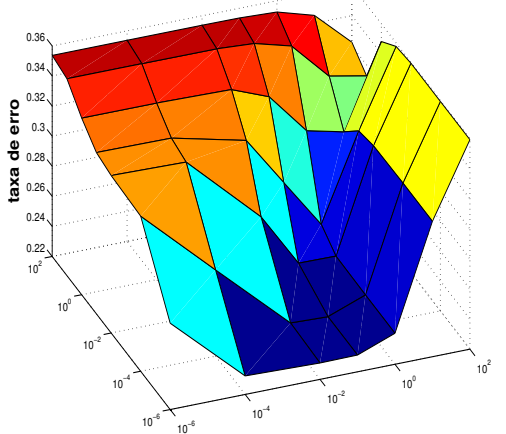

(c) symFKNN-RBF

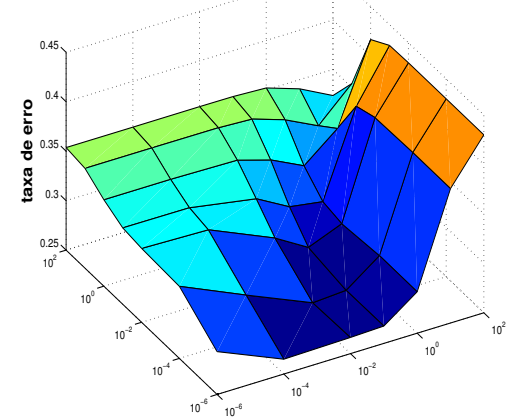

(f) symFKNN-HM

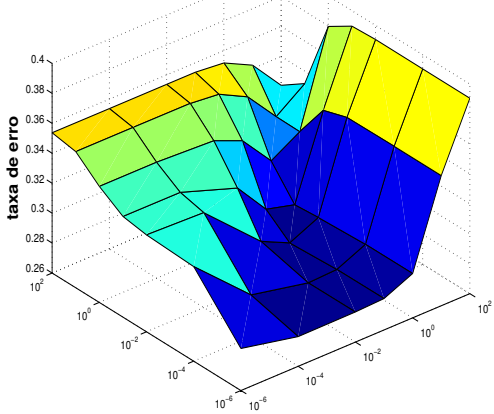

(i) SymFKNN-LLE

Figura 7.25: Superfícies de erro do algoritmo LapSVM na base de dados TEXT para as partições de 100 exemplos rotulados. 


\subsection{Avaliação dos resultados obtidos}

A partir da análise dos resultados obtidos, pode-se extrair as seguintes constatações empíricas:

- Na Figura 7.2, nota-se que o grafo mutKNN gerou superfícies de erro mais suaves que as geradas pelos grafos symKNN e symFKNN. Essa é uma propriedade interessante em aplicações reais. Os grafos symKNN e symFKNN geraram superfícies de erro bastante similares;

- Na Figura 7.3, nota-se que as superfícies de erro geradas a partir do kernel RBF são bastante diferentes das demais. Para os métodos HM e LLE, nota-se que os grafos symKNN e symFKNN geraram melhores superfícies de erro que o grafo mutKNN. Entretanto, o grafo mutKNN apresentou melhores desempenhos de classificação que os demais grafos. Ainda mais, os grafos symKNN e symFKNN apresentaram alta instabilidade quando $\gamma_{A}>\gamma_{I}$ ao se usar os métodos HM e LLE;

- Na Figura 7.4, nota-se que todas as superfícies de erro apresentaram comportamentos similares. A região "ótima” ocorre quando $\gamma_{A}>\gamma_{I}$;

- Na Figura 7.5, chega-se às mesmas conclusões apresentadas para a Figura 7.3;

- Nas Figuras 7.6 e 7.8, nota-se que o grafo mutKNN gerou superfícies de erro mais suaves que as geradas pelos grafos symKNN e symFKNN;

- Nas Figuras 7.7 e 7.9, nota-se que todos os grafos geraram superfícies de erro bastante similares;

- Nas Figuras 7.10 até 7.13, nota-se que todos os grafos geraram superfícies de erro bastante similares. A região "ótima" ocorre quando $\gamma_{A}>\gamma_{I}$;

- Nas Figuras 7.14 até 7.21 , nota-se que a região "ótima” ocorre quando $\gamma_{A}<\gamma_{I}$ e todos os grafos geraram superfícies de erro bastante similares;

- Na Figura 7.22, observa-se que a região "ótima" ocorre quando $\gamma_{A}=\gamma_{I}$ para todos os grafos. Quando $\gamma_{A} \neq \gamma_{I}$, os resultados não foram satisfatórios;

- Na Figura 7.23, nota-se que todos os grafos apresentaram superfícies de erro similares quando $\gamma_{A}>\gamma_{I}$. Quando $\gamma_{A}<\gamma_{I}$, as superfícies de erro geradas a partir do kernel RBF apresentaram uma pequena instabilidade, enquanto que as superfícies de erro referentes aos métodos HM e LLE apresentaram resultados estáveis. Entretanto, os resultados referentes ao kernel RBF são melhores que os referentes aos métodos HM e LLE;

- Na Figura 7.24, observa-se que as superfícies de erro geradas a partir do kernel RBF são bastante similares. Entretanto, parece não haver um 
padrão evidente de comportamento para os demais grafos. Para exemplificar, nota-se que a região "ótima" para o grafo mutKNN-LLE ocorre quando $\gamma_{A}=\gamma_{I}$, enquanto que essa configuração não é adequada para os grafos symKNN-RBF, mutKNN-RBF, symFKNN-RBF e mutKNN-HM;

- Na Figura 7.25, observa-se que as superfícies de erro geradas por todos os grafos são bastante similares.

\subsection{Considerações finais}

Neste capítulo, propôs-se um modelo de avaliação experimental para avaliar a influência que os parâmetros de regularização apresentam no desempenho de classificação dos algoritmos de aprendizado semissupervisionado. A partir da análise dos resultados, constatou-se que os resultados obtidos tem uma forte dependência em relação ao valor dos parâmetros de regularização, o que dificulta o processo de escolha de parâmetros. As regiões "ótimas" variaram para cada base de dados, o que impossibilitou tirar conclusões gerais no processo de análise dos resultados.

Acredita-se que o modelo de avaliação experimental proposto neste capítulo deva ser amplamente usado na literatura de aprendizado semissupervisionado. Com o uso desse modelo de avaliação experimental, a quantidade de conclusões enviesadas referentes à avaliação dos parâmetros de regularização seria drasticamente reduzida, o que aumentaria a maturidade empírica da área de aprendizado semissupervisionado. O modelo de avaliação experimental proposto neste capítulo pode ser usado em outras áreas do aprendizado de máquina, não se restringindo à classificação de dados. Por exemplo, pode-se analisar a influência dos parâmetros de qualquer algoritmo em relação a uma dada medida a partir da análise das superfícies de erro geradas. Com isso, o modelo proposto neste capítulo poderá ser usado em diversos trabalhos empíricos na área de aprendizado de máquina. Acredita-se que o uso do modelo proposto neste capítulo possa melhorar a qualidade dos trabalhos empíricos na área de aprendizado de máquina. 


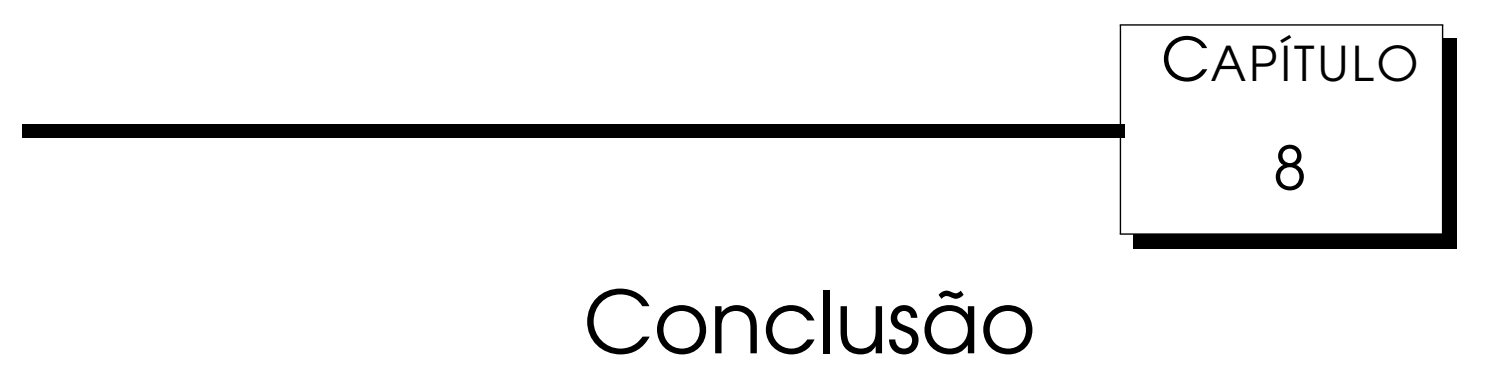

Neste trabalho, três modelos de avaliação experimental que visam analisar a estabilidade de classificadores e métodos de geração de grafos foram propostos. Alguns dos resultados e análises experimentais neste trabalho foram aceitos para publicação (de Sousa et al., 2013).

Neste trabalho, fez-se uma avaliação empírica detalhada de cinco algoritmos de aprendizado semissupervisionado baseado em grafos combinados com três métodos de geração de grafos de adjacência e três de geração de matrizes ponderadas. Foram propostos neste trabalho três modelos de avaliação experimental. Acredita-se também que tais métodos possam ser amplamente usados na área de aprendizado de máquina para melhorar a qualidade do processo de análise experimental. Em adição, outros modelos de avalição experimental poderiam ser criados a partir dos propostos neste trabalho.

Neste trabalho, é proposto um protocolo experimental que deve ser usado em avaliações experimentais em trabalhos futuros na área de aprendizado semissupervisionado. A ideia chave é que os novos algoritmos propostos não deveriam ser comparados com os métodos do estado da arte com base apenas na análise de melhor caso. Ainda mais, acredita-se que uma avaliação detalhada da influência de todos os parâmetros no desempenho de classificação dos algoritmos seja necessária não apenas para o aprendizado semissupervisionado, mas para todas as àreas do aprendizado de máquina.

A avaliação experimental feita neste trabalho indica que os algoritmos de aprendizado semissupervisionado são fortemente afetados pelo valores dos parâmetros de esparsificação e de regularização, bem como pela escolha do método de geração de grafos. Em adição, os classificadores semissupervisionados que apresentam parâmetros de regularização são extremamente dependentes de uma boa configuração desses parâmetros.

Por causa da natureza do aprendizado semissupervisionado, em que assumese que haja apenas uma pequena quantidade de exemplos rotulados, calibrar todos os parâmetros pode ser uma tarefa inviável. Logo, há necessidade da criação de classificadores semissupervisionados que sejam fracamente depen- 
dentes do processo de escolha de parâmetros, ou seja, que sejam estáveis no espaço de parâmetros.

A partir dos resultados experimentais obtidos neste trabalho, nota-se a superioridade do grafo mutKNN em comparação aos grafos symKNN e symFKNN. Entretanto, esses resultados também indicam que o grafo mutKNN é instável para valores relativamente pequenos de $k$. Em adição, percebeu-se que os grafos mutKNN tendem a gerar superfícies de erro que são mais suaves que aquelas geradas pelos grafos symKNN e symFKNN. As análises estatísticas dos resultados apontaram tanto a superioridade do grafo mutKNN em comparação aos grafos symKNN e symFKNN, quanto a superioridade do kernel RBF em comparação aos métodos HM e LLE.

Ao analisar os resultados obtidos, foram encontrados alguns padrões interessantes que não puderam ser verificados dada a falta de evidência experimental. Discute-se a seguir essas observações e propõem-se a investigação da validade das mesmas em trabalhos futuros. A partir dos resultados obtidos, chegam-se às seguintes suposições:

- Apesar de o algoritmo RMGT obter resultados satisfatórios na maioria das bases de dados, ele não apresentou um bom desempenho de classificação na base USPS. Como a base USPS é desbalanceada, uma possível explicação para esse desempenho insatisfatório é que o algoritmo RMGT pode não ser efetivo em bases de dados com rótulos desbalanceados. Um estudo experimental envolvendo esse tópico poderia verificar a validade dessa hipótese;

- Maier et al. (2009a) apontaram que o grafo mutKNN deveria ser escolhido quando há apenas o interesse em identificar o agrupamento "mais significativo". Baseado nessa assertiva, supõe-se que o grafo mutKNN é o melhor grafo ao se lidar com bases de dados com rótulos desbalanceados tendo em vista que ele poderia identificar a classe "mais significativa" (a classe minoritária nesse caso). Essa hipótese é suportada pelo fato de o grafo mutKNN obter o melhor desempenho de classificação que os grafos symKNN e symFKNN para todas as combinações de algoritmo semissupervisionado e método de geração de matrizes ponderadas na base USPS, conforme mostrado na Tabela 4.1. Para as partições de 100 exemplos rotulados, o grafo mutKNN obteve o melhor desempenho de classificação na maioria dos casos e um desempenho competitivo nos demais casos, conforme mostrado na Tabela 4.3;

- Na Tabela 4.1, mostra-se que o algoritmo RMGT obteve o melhor desempenho de classificação global em quatro das seis bases de dados. Na Tabela 4.3, nota-se que o algoritmo RMGT obteve o melhor desempenho de classificação em duas bases de dados e resultados competitivos nas outras bases de dados. Esse desempenho de classificação surpreendente pode ser explicado pela adição das restrições de normalização $\boldsymbol{F} \mathbf{1}_{c}=\mathbf{1}_{n}$ e $\boldsymbol{F}^{\top} \mathbf{1}_{n}=n \boldsymbol{\omega}$ no modelo de otimização. Seria interessante investigar se os demais classificadores teriam um melhor desempenho de classificação ao se incorporar essas restrições nos seus modelos de otimização; 
- Na Figura 7.22, observa-se que a "região ótima" ocorre apenas quando $\gamma_{A}=\gamma_{I}$. Como este comportamento ocorreu para todos os grafos, supõese que essa configuração deva ser escolhida para tarefas de classificação de textos ao usar o algoritmo LapRLS. Um estudo empírico na área de classificação de textos semissupervisionada poderia verificar a validade dessa hipótese.

Em suma, abordou-se neste trabalho a avaliação do impacto da geração de grafos na classificação semissupervisionada usando quatro modelos de avaliação empírica. Vários dos resultados obtidos neste trabalho apresentam pontos negativos para a área de aprendizado semissupervisionado. Acredita-se que ao se usar os modelos experimentais propostos neste trabalho, a área de aprendizado semissupervisionado, bem como outras áreas do aprendizado de máquina, poderia amadurecer bastante. A partir da geração desses modelos, pode-se concluir os objetivos deste trabalho e validar a hipótese de que é possivel avaliar de maneira detalhada o impacto da geração de grafos na classificação semissupervisionada. Com o uso desses modelos experimentais, as avaliações empíricas descritas nos trabalhos científicos poderiam ser mais detalhadas e as conclusões obtidas podem levar a insights sobre o processo de escolha de classificadores e métodos de geração de grafos para aplicações reais, bem como direções para trabalhos futuros. 



\section{Referências Bibliográficas}

Argyriou, A., Herbster, M., e Pontil, M. (2005). Combining graph laplacians for semi-supervised learning. In Advances in Neural Information Processing Systems 18, páginas 67-74. Citado na página 1.

Balcan, M.-F. e Blum, A. (2005). A pac-style model for learning from labeled and unlabeled data. In In Proceedings of the 18th Annual Conference on Computational Learning Theory, páginas 111-126. Citado na página 1.

Balcan, M.-F. e Blum, A. (2010). A discriminative model for semi-supervised learning. Journal of the ACM, 57(3):1-46. Citado na página 1.

Belkin, M., Niyogi, P., e Sindhwani, V. (2006). Manifold regularization: A geometric framework for learning from labeled and unlabeled examples. Journal of Machine Learning Research, 7:2399-2434. Citado nas páginas 1, 3, 13, 14 , e 15.

Bengio, Y., Paiement, J.-F., Vincent, P., Delalleau, O., Roux, N. L., e Ouimet, M. (2004). Out-of-sample extensions for LLE, Isomap, MDS, Eigenmaps, and spectral clustering. In Advances in Neural Information Processing Systems 16, páginas 177-184. Citado na página 15.

Beyer, K., Goldstein, J., Ramakrishnan, R., e Shaft, U. (1999). When is "nearest neighbor"meaningful? In Proceedings of the International Conference on Databse Theory, páginas 217-235. Citado na página 18.

Blum, A. e Chawla, S. (2001). Learning from labeled and unlabeled data using graph mincuts. In Proceedings of the International Conference on Machine Learning, páginas 19-26. Citado na página 1.

Blum, A., Lafferty, J., Rwebangira, M. R., e Reddy, R. (2004). Semi-supervised learning using randomized mincuts. In Proceedings of the International Conference on Machine learning, páginas 13-20. Citado na página 1.

Chapelle, O., Schölkopf, B., e Zien, A. (2006). Semi-supervised learning. The MIT Press. Citado nas páginas xi, 1, 2, 7, 8, 17, 18, 23, 27, e 28. 
Cortes, C., Mohri, M., Pechyony, D., e Rastogi, A. (2008). Stability of transductive regression algorithms. In Proceedings of the International Conference on Machine Learning, páginas 176-183. Citado na página 1.

Daitch, S., Kelner, J., e Spielman, D. (2009). Fitting a graph to vector data. In Proceedings of the International Conference on Machine Learning, páginas 201-208. Citado na página 1.

de Sousa, C. A. R. (2012). Analysis of the backpropagation algorithm using linear algebra. In Proceedings of the IEEE International Joint Conference on Neural Networks, páginas 7-14. Citado na página 15.

de Sousa, C. A. R., Rezende, S. O., e Batista, G. E. A. P. A. (2013). Influence of graph construction on semi-supervised learning. In Proceedings of the European Conference on Machine Learning and Principles and Practice of Knowledge Discovery in Databases (ECML PKDD) (no prelo). Citado na página 87.

Delalleau, O., Bengio, Y., e Roux, N. (2005). Efficient non-parametric function induction in semi-supervised learning. In Proceedings of the International Conference on Artificial Intelligence and Statistics, páginas 96-103. Citado na página 15.

Demšar, J. (2006). Statistical comparisons of classifiers over multiple data sets. Journal of Machine Learning Research, 7:1-30. Citado na página 26.

Derbeko, P., El-Yaniv, R., e Meir, R. (2004). Error bounds for transductive learning via compression and clustering. In Advances in Neural Information Processing Systems (NIPS) 16, páginas 1085-1092. Citado na página 1.

Ghazvininejad, M., Mahdieh, M., Rabiee, H. R., Roshan, P. K., e Rohban, M. H. (2011). Isograph: Neighbourhood graph construction based on geodesic distance for semi-supervised learning. In Proceedings of the IEEE International Conference on Data Mining, páginas 191-200. Citado nas páginas 1, 7, e 23.

Hastie, T., Tibshirani, R., e Friedman, J. (2009). The Elements of Statistical Learning: Data Mining, Inference, and Prediction. Springer, $2^{\text {a }}$ edição. Citado na página 15.

Hein, M., Audibert, J.-Y., e von Luxburg, U. (2007). Graph laplacians and their convergence on random neighborhood graphs. Journal of Machine Learning Research, 8:1325-1368. Citado na página 9.

Hein, M. e Maier, M. (2007). Manifold denoising. In Advances in Neural Information Processing Systems 19, páginas 561-568. Citado nas páginas 1, 11 , e 41 .

Huang, B. e Jebara, T. (2007). Loopy belief propagation for bipartite maximum weight b-matching. In Proceedings of the International Conference on Artificial Intelligence and Statistics, páginas 195-202. Citado na página 21. 
Huang, B. e Jebara, T. (2011). Fast b-matching via sufficient selection belief propagation. In Proceedings of the International Conference on Artificial Intelligence and Statistics, páginas 361-369. Citado na página 21.

Jebara, T., Wang, J., e Chang, S.-F. (2009). Graph construction and bmatching for semi-supervised learning. In Proceedings of the International Conference on Machine Learning, páginas 441-448. Citado na página 21.

Johnson, R. e Zhang, T. (2007). On the effectiveness of laplacian normalization for graph semi-supervised learning. Journal of Machine Learning Research, 8:1489-1517. Citado nas páginas 1 e 19.

Kääriäinen, M. (2005). Generalization error bounds using unlabeled data. In Proceedings of the 18th Annual Conference on Learning Theory, páginas 127142. Citado na página 1.

Kong, D., Ding, C., Huang, H., e Nie, F. (2012). An iterative locally linear embedding algorithm. In Proceedings of the International Conference on Machine Learning, páginas 1647-1654. Citado nas páginas 1, 7, e 23.

Lafferty, J. e Wasserman, L. (2008). Statistical analysis of semi-supervised regression. In Advances in Neural Information Processing Systems, páginas 801-808. Citado na página 1.

Liu, W. e Chang, S.-F. (2009). Robust multi-class transductive learning with graphs. In Proceedings of the IEEE International Conference on Computer Vision and Pattern Recognition, páginas 381-388. Citado nas páginas 1, 3, $11,14,22$, e 41 .

Liu, W., He, J., e Chang, S.-F. (2010). Large graph construction for scalable semi-supervised learning. In Proceedings of the International Conference on Machine Learning, páginas 679-686. Citado nas páginas 1 e 19.

Luo, D., Ding, C., Huang, H., e Nie, F. (2012). Forging the graphs: A low rank and positive semidefinite graph learning approach. In Advances in Neural Information Processing Systems 25, páginas 2969-2977. Citado nas páginas $1,2,7,8$, e 23 .

Maier, M., Hein, M., e von Luxburg, U. (2007). Cluster identification in nearest-neighbor graphs. In Proceedings of the Conference on Learning Theory, páginas 196-210. Citado na página 11.

Maier, M., Hein, M., e von Luxburg, U. (2009a). Optimal construction of $k$ nearest-neighbor graphs for identifying noisy clusters. Theoretical Computer Science, 410(19):1749-1764. Citado nas páginas 11 e 88.

Maier, M., Luxburg, U. V., e Hein, M. (2009b). Influence of graph construction on graph-based clustering measures. In Advances in Neural Information Processing Systems 21, páginas 1025-1032. Citado na página 11. 
Melacci, S. e Belkin, M. (2011). Laplacian support vector machines trained in the primal. Journal of Machine Learning Research, 12:1149-1184. Citado nas páginas $1,20,22$, e 23.

Nie, F., Wang, H., Huang, H., e Ding, C. (2011). Unsupervised and semisupervised learning via $l_{1}$-norm graph. In Proceedings of the International Conference on Computer Vision and Pattern Recognition, páginas 2268-2273. Citado nas páginas 1, 7, e 23.

Ozaki, K., Shimbo, M., Komachi, M., e Matsumoto, Y. (2011). Using the mutual k-nearest neighbor graphs for semi-supervised classification of natural language data. In Proceedings of the Conference on Computational Natural Language Learning, páginas 154-162. Citado na página 19.

Rigollet, P. (2007). Generalization error bounds in semi-supervised classification under the cluster assumption. Journal of Machine Learning Research, 8:1369-1392. Citado na página 1 .

Roweis, S. e Saul, L. (2000). Nonlinear dimensionality reduction by locally linear embedding. Science, 290(5500):2323-2326. Citado nas páginas 1, 11 , e 12 .

Saul, L. K. e Roweis, S. T. (2003). Think globally, fit locally: unsupervised learning of low dimensional manifolds. Journal of Machine Learning Research, 4:119-155. Citado na página 12 .

Sindhwani, V., Niyogi, P., e Belkin, M. (2005). Beyond the point cloud: from transductive to semi-supervised learning. In Proceedings of the International Conference on Machine Learning, páginas 824-831. C Citado na página 1.

Tong, W. e Jin, R. (2007). Semi-supervised learning by mixed label propagation. In Proceedings of the 22nd national conference on Artificial intelligence Volume 1, páginas 651-656. Citado na página 1.

Urner, R., Shalev-Shwartz, S., e Ben-David, S. (2011). Access to unlabeled data can speed up prediction time. In Proceedings of the 28th International Conference on Machine Learning, páginas 641-648. Citado nas páginas 1, 7,8 , e 23.

Wang, J., Chang, S.-F., Zhou, X., e Wong, S. T. C. (2008). Active microscopic cellular image annotation by superposable graph transduction with imbalanced labels. In Proceedings of the IEEE International Conference on Computer Vision and Pattern Recognition, páginas 1-8. Citado na página 21.

Wang, J. e Xia, Y. (2012). Fast graph construction using auction algorithm. In Proceedings of the Conference on Uncertainty in Artificial Intelligence, páginas 873-882. Citado nas páginas 1, 2, 7, 8, e 23.

Wu, X.-M., Li, Z., So, A. M.-C., Wright, J., e Chang, S.-F. (2012). Learning with partially absorbing random walks. In Advances in Neural Information 
Processing Systems 25, páginas 3086-3094. Citado nas páginas 1, 2, 7, e 8 .

Yang, H., Zhu, S., King, I., e Lyu, M. R. (2011). Can irrelevant data help semisupervised learning, why and how? In Proceedings of the ACM International Conference on Information and Knowledge Management, páginas 937-946. Citado nas páginas 1,7 , e 8 .

Zelnik-Manor, L. e Perona, P. (2004). Self-tuning spectral clustering. In Advances in Neural Information Processing Systems 17, páginas 1601-1608. Citado na página 21.

Zhang, T. e Ando, R. (2006). Analysis of spectral kernel design based semisupervised learning. In Advances in Neural Information Processing Systems 18, páginas 1601-1608. Citado na página 1 .

Zhang, X. e Lee, W. (2007). Hyperparameter learning for graph based semisupervised learning algorithms. In Advances in Neural Information Processing Systems 19, páginas 1585-1592. Citado na página 1.

Zhang, Y. e Zhou, Z.-H. (2009). Non-metric label propagation. In Proceedings of the 21st International Joint Conference on Artifical Intelligence, páginas 1357-1362. Citado na página 1.

Zhang, Y.-M., Huang, K., e Liu, C.-L. (2011). Fast and robust graph-based transductive learning via minimum tree cut. In Proceedings of the IEEE International Conference on Data Mining, páginas 952-961. Citado nas páginas $1,7,8$, e 23.

Zhou, D., Bousquet, O., Lal, T., Weston, J., e Schölkopf, B. (2004). Learning with local and global consistency. In Advances in Neural Information Processing Systems 16, páginas 321-328. Citado nas páginas 1 e 13.

Zhu, X. (2005). Semi-supervised learning literature survey. Relatório Técnico 1530, Computer Sciences, University of Wisconsin-Madison. Citado nas páginas $1,2,4,7,8,9,23,40$, e 41 .

Zhu, X., Ghahramani, Z., e Lafferty, J. (2003). Semi-supervised learning using gaussian fields and harmonic functions. In Proceedings of the International Conference on Machine Learning, páginas 912-919. Citado nas páginas 1, 12,20 , e 21.

Zhuang, L., Gao, H., Lin, Z., Ma, Y., Zhang, X., e Yu, N. (2012). Non-negative low rank and sparse graph for semi-supervised learning. In Proceedings of the International Conference on Computer Vision and Pattern Recognition, páginas 2328-2335. Citado nas páginas 1, 2, 7, 8, e 23. 